\title{
Sequência exata de Bloch-Wigner e K-teoria algébrica
}

\author{
David Martín Carbajal Ordinola
}



Data de Depósito:

Assinatura:

\title{
David Martín Carbajal Ordinola
}

\section{Sequência exata de Bloch-Wigner e K-teoria algébrica}

\begin{abstract}
Dissertação apresentada ao Instituto de Ciências Matemáticas e de Computação - ICMC-USP, como parte dos requisitos para obtenção do título de Mestre em Ciências - Matemática. VERSÃO REVISADA
\end{abstract}

Área de Concentração: Matemática

Orientador: Prof. Dr. Behrooz Mirzaii 
Ficha catalográfica elaborada pela Biblioteca Prof. Achille Bassi e Seção Técnica de Informática, ICMC/USP, com os dados fornecidos pelo(a) autor(a)

\begin{tabular}{|c|c|}
\hline OC263s & $\begin{array}{l}\text { Ordinola, David Martín Carbajal } \\
\text { Sequência exata de Bloch-Wigner e K-teoria } \\
\text { algébrica / David Martín Carbajal Ordinola; } \\
\text { orientador Behrooz Mirzaii. - São Carlos - SP, } 2016 . \\
\quad 111 \text { p. }\end{array}$ \\
\hline & $\begin{array}{l}\text { Dissertação (Mestrado - Programa de Pós-Graduação } \\
\text { em Matemática) - Instituto de Ciências Matemáticas e } \\
\text { de Computação, Universidade de São Paulo, } 2016 . \\
\text { 1. Sequência exata de Bloch-Wigner. 2. K-grupos } \\
\text { de Quillen. 3. K-teoria algébrica. 4. Sequências } \\
\text { espectrais.. I. Mirzaii, Behrooz, orient. II. Título. }\end{array}$ \\
\hline
\end{tabular}




\section{David Martín Carbajal Ordinola}

\section{The Bloch-Wigner exact sequence and algebraic K-theory}

Master dissertation submitted to the Instituto de Ciências Matemáticas e de Computação - ICMCUSP, in partial fulfillment of the requirements for the degree of the Master Program in Mathematics. FINAL VERSION

Concentration Area: Mathematics

Advisor: Prof. Dr. Behrooz Mirzaii

USP - São Carlos

October, 2016 

Dedico este trabalho:

- a Deus, pela fé que me mantém vivo e fiel à vida honesta de trabalho e de estudo;

- à minha vó Alvina Quepuy M., por sua paciência, por seu amor e por valorizar meu esforço; estarei eternamente grato;

- à minha familia que soube entender a minha ausência nos muitos momentos desde que ingressei no mestrado no exterior até a conclusão desta dissertação;

- a todos os professores do ICMC que me apoiaram e acreditaram em mim durante o mestrado e no momento de escrever a dissertação;

- ao Instituto de Ciências Matemáticas e de Computação (ICMC), por acreditar no meu profissionalismo e trabalho. 

Esta dissertação contou com a disponibilidade, apoio e colaboração de um grupo de pessoas, e para elas o meu reconhecimento nesta página, sobretudo por me incentivarem a ultrapassar constrangimentos nesta fase difícil da minha vida.

Manifesto a minha gratidão ao Professor Doutor Behrooz Mirzaii, orientador desta dissertação, por sua paciência e confiança demonstrada desde nosso primeiro encontro, pelas críticas, conselhos e, sobretudo pelo estímulo e ajuda na concretização desta dissertação.

Agradeço em particular a todos os meus professores do mestrado, cujos ensinamentos me permitiram conduzir esta dissertação, proporcionando-me experiências muito significativas, pois dispensaram parte de seu tempo para responder minhas dúvidas.

Agradeço a minha família que me faz acreditar todos os dias que vale a pena estudar no exterior, investindo muito tempo nesta árdua tarefa e assim oferecer o melhor de mim. Eles constituem minha razão de querer ser sempre melhor.

Finalmente, agradeço aos meus amigos peruanos e brasileiros que me ajudaram a cumprir com sucesso o mestrado e ofereceram sempre seu apoio ao longo destes dois anos.

David Martín Carbajal Ordinola 

"It is not knowledge, but the act of learning, not possession but the act of getting there, which grants the greatest enjoyment. When I have clarified and exhausted a subject, then I turn away from it, in order to go into darkness again..." (Johann Carl Friedrich Gauss) 



\section{RESUMO}

DAVID M. CARBAJAL ORDINOLA. Sequência exata de Bloch-Wigner e K-teoria algébrica. 2016. 111 f. Dissertação (Mestrado em Ciências - Matemática) - Instituto de Ciências Matemáticas e de Computação (ICMC/USP), São Carlos - SP.

A $K$-teoria algébrica é um ramo da álgebra que associa para cada anel com unidade $R$, uma sequência de grupos abelianos chamados os $n$-ésimos $K$-grupos de $R$. Em 1970, Daniel Quillen dá uma definição geral dos $K$-grupos de um anel qualquer $R$ a partir da +-construção do espaço classificante $B G L(R)$. Por outro lado, considerando $R$ um anel comutativo, obtém-se também a definição dos $K$-grupos de Milnor $K_{n}^{M}(R)$.

Usando o produto dos $K$-grupos de Quillen e Milnor e suas estruturas anti-comutativas, definimos o seguinte homomorfismo

$$
\tau_{n}: K_{n}^{M}(R) \rightarrow K_{n}(R)
$$

Mostraremos nesta dissertação que se $R$ é um anel local com ideal maximal $\mathfrak{m}$ tal que $R / \mathfrak{m}$ é um corpo infinito, então esse homomorfismo é um isomorfismo para $0 \leq n \leq 2$. Em geral $\tau_{n}$ nem sempre é injetor ou sobrejetor. Por exemplo quando $n=3$, sabe-se que $\tau_{3}$ não é sobrejetor e definimos a parte indecomponível de $K_{3}(R)$ como sendo o grupo

$$
K_{3}^{\text {ind }}(R):=\operatorname{coker}\left(K_{3}^{M}(R) \stackrel{\tau_{3}}{\rightarrow} K_{3}(R)\right) .
$$

Usando alguns resultados de homologia dos grupos lineares, nesta dissertação mostraremos a existência da sequência exata de Bloch-Wigner para corpos infinitos. Esta sequência dá uma descrição explícita da parte indecomponível do terceiro $K$-grupo de um corpo infinito.

TEOREMA (Sequência exata de Bloch-Wigner). Seja $F$ um corpo infinito e seja $\mathfrak{p}(F)$ o grupo de pre-Bloch de $F$, isto é, o grupo quociente do grupo abeliano livre gerado pelos símbolos $[a]$, $a \in F^{\times}-\{1\}$, pelo subgrupo gerado por elementos da forma

$$
[a]-[b]+\left[\frac{b}{a}\right]-\left[\frac{1-a^{-1}}{1-b^{-1}}\right]+\left[\frac{1-a}{1-b}\right]
$$

$\operatorname{com} a, b \in F^{\times}-\{1\}, a \neq b$. Então temos a sequência exata

$$
\operatorname{Tor}_{1}^{\mathbb{Z}}(\mu(F), \mu(F))^{\sim} \rightarrow K_{3}^{\text {ind }}(F) \rightarrow \mathfrak{p}(F) \rightarrow\left(F^{\times} \otimes_{\mathbb{Z}} F^{\times}\right)_{\sigma} \rightarrow K_{2}(F) \rightarrow 0
$$

onde $\left(F^{\times} \otimes_{\mathbb{Z}} F^{\times}\right)_{\sigma}:=\left(F^{\times} \otimes_{\mathbb{Z}} F^{\times}\right) /\left\langle a \otimes b+b \otimes a \mid a, b \in F^{\times}\right\rangle$e $\operatorname{Tor}_{1}^{\mathbb{Z}}(\mu(F), \mu(F))^{\sim}$ é a única extensão não trivial de $\mathbb{Z} / 2 \mathbb{Z}$ por $\operatorname{Tor}_{1}^{\mathbb{Z}}(\mu(F), \mu(F))$ se $\operatorname{char}(F) \neq 2$ e $\mu_{2^{\infty}}(F)$ é finito e é $\operatorname{Tor}_{1}^{\mathbb{Z}}(\mu(F), \mu(F))$ caso contrário. O homomorfismo $\mathfrak{p}(F) \rightarrow\left(F^{\times} \otimes_{\mathbb{Z}} F^{\times}\right)_{\sigma}$ é definido por $[a] \mapsto a \otimes(1-a)$. 
O estudo da sequência exata de Bloch-Wigner é justificada pela relação entre o segundo e terceiro $K$-grupo de um corpo $F$.

Palavras-chave: Sequência exata de Bloch-Wigner, K-grupos de Quillen, K-teoria algébrica, Sequências espectrais.. 


\section{ABSTRACT}

DAVID M. CARBAJAL ORDINOLA. Sequência exata de Bloch-Wigner e K-teoria algébrica. 2016. 111 f. Dissertação (Mestrado em Ciências - Matemática) - Instituto de Ciências Matemáticas e de Computação (ICMC/USP), São Carlos - SP.

The algebraic $K$-theory is a branch of algebra that associates to any ring with unit $R$ a sequence of abelian groups called $n$-th $K$-groups of $R$. In 1970, Daniel Quillen gave a general definition of $K$-groups of any ring $R$ using the +-construction of the classifying space $B G L(R)$. On the other hand, if we consider a commutative ring $R$, we can define the Milnor's $K$-groups, $K_{n}^{M}(R)$, of $R$.

Using the product of the Quillen and Milnor's $K$-groups and their anti-commutative structure, we define a natural homomorphism

$$
\tau_{n}: K_{n}^{M}(R) \rightarrow K_{n}(R)
$$

In this dissertation, we show that if $R$ is a local ring with maximal ideal $\mathfrak{m}$ such that $R / \mathfrak{m}$ is infinite, then this map is an isomorphism for $0 \leq n \leq 2$. But in general $\tau_{n}$ is not injective nor is surjective. For example when $n=3$, we know that $\tau_{3}$ is not surjective and define the indecomposable part of $K_{3}(R)$ as the group

$$
K_{3}^{\text {ind }}(R):=\operatorname{coker}\left(K_{3}^{M}(R) \stackrel{\tau_{3}}{\rightarrow} K_{3}(R)\right) .
$$

Using some results about the homology of linear groups, in this dissertation we will prove the Bloch-Wigner exact sequence over infinite fields. This exact sequence gives us a precise description of the indecomposable part of the third $K$-group of an infinite field.

THEOREM (Bloch-Wigner exact sequence). Let $F$ be an infinite field and let $\mathfrak{p}(F)$ be the pre-Bloch group of $F$, that is, the quotient group of the free abelian group generated by symbols $[a], a \in F^{\times}-\{1\}$, by the subgroup generated by the elements of the form

$$
[a]-[b]+\left[\frac{b}{a}\right]-\left[\frac{1-a^{-1}}{1-b^{-1}}\right]+\left[\frac{1-a}{1-b}\right]
$$

with $a, b \in F^{\times}-\{1\}, a \neq b$. Then we have the exact sequence

$$
\operatorname{Tor}_{1}^{\mathbb{Z}}(\mu(F), \mu(F))^{\sim} \rightarrow K_{3}^{\text {ind }}(F) \rightarrow \mathfrak{p}(F) \rightarrow\left(F^{\times} \otimes_{\mathbb{Z}} F^{\times}\right)_{\sigma} \rightarrow K_{2}(F) \rightarrow 0
$$

where $\left(F^{\times} \otimes_{\mathbb{Z}} F^{\times}\right)_{\sigma}:=\left(F^{\times} \otimes_{\mathbb{Z}} F^{\times}\right) /\left\langle a \otimes b+b \otimes a \mid a, b \in F^{\times}\right\rangle$and $\operatorname{Tor}_{1}^{\mathbb{Z}}(\mu(F), \mu(F))^{\sim}$ is the unique non trivial extension of $\mathbb{Z} / 2 \mathbb{Z}$ by $\operatorname{Tor}_{1}^{\mathbb{Z}}(\mu(F), \mu(F))$ if $\operatorname{char}(F) \neq 2$ and $\mu_{2^{\infty}}(F)$ is finite and is $\operatorname{Tor}_{1}^{\mathbb{Z}}(\mu(F), \mu(F))$ otherwise. The homomorphism $\mathfrak{p}(F) \rightarrow\left(F^{\times} \otimes_{\mathbb{Z}} F^{\times}\right)_{\sigma}$ is defined by $[a] \mapsto a \otimes(1-a)$. 
As it is shown, the study of the Bloch-Wigner exact sequence is also justified by the relation between the second and third $K$-group of a field $F$.

Key-words: Bloch-Wigner exact sequence, Quillen's K-groups, Algebraic K-theory, Spectral sequences.. 


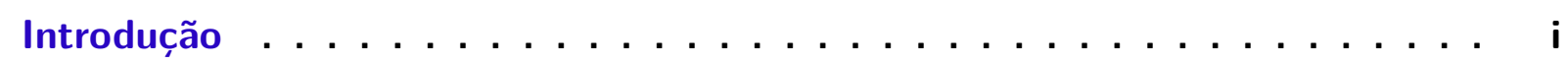

1 HOMOLOGIA DE GRUPOS ................... . . 1

$1.1 \quad$ Complexos de cadeias $\ldots \ldots \ldots \ldots \ldots$

$1.2 \quad$ Homologia de Grupos . . . . . . . . . . . . . . . 7

$1.3 \quad 0$ grupo $\operatorname{Ext}_{\mathbb{Z}}^{1}(B, A) \ldots \ldots \ldots \ldots \ldots$

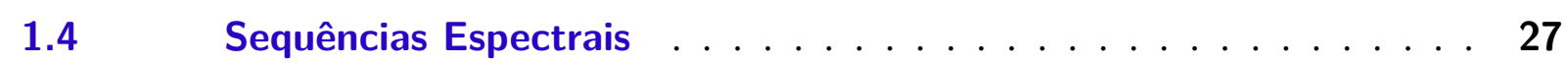

$1.5 \quad$ Homologia dos grupos lineares . . . . . . . . . . . . 38

$2 \quad K_{2}$ DE ANÉIS E O TEOREMA DE MATSUMOTO . . . . . . 41

$2.1 \quad K_{0}(R)$ e $K_{1}(R) \ldots \ldots \ldots \ldots \ldots \ldots \ldots \ldots \ldots \ldots$

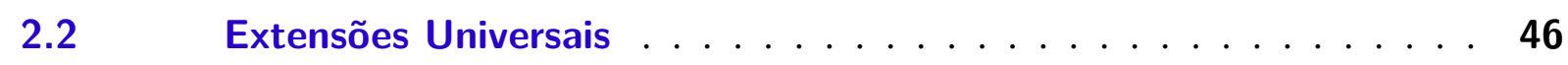

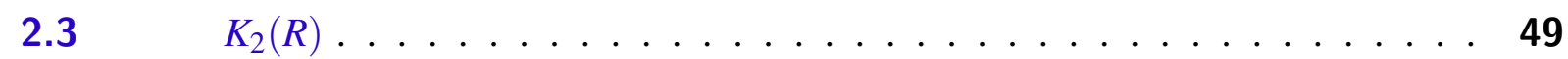

$2.4 \quad$ Sequência Espectral Principal . . . . . . . . . . . . . . . 52

$2.5 \quad 0$ teorema de Van der Kallen-Matsumoto . . . . . . . . . 55

$3 \quad K$-TEORIA ALGÉBRICA DE ANÉIS . . . . . . . . . . 67

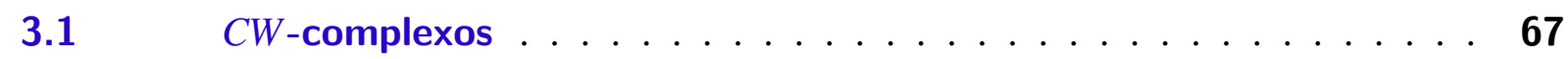

$3.2 \quad$ A + -construção $\ldots \ldots \ldots \ldots \ldots \ldots$

$3.3 \quad K$-teoria de Quillen e Milnor . . . . . . . . . . . . . 76

$3.4 \quad \mathrm{O}$ produto nos $K$-grupos de Quillen $\ldots \ldots \ldots \ldots$

$4 \quad K_{3}$ DE CORPOS E O TEOREMA DE BLOCH-WIGNER . . . . . 87

4.1 Terceira homologia de $G L_{2}$ e o grupo de Bloch . . . . . . . . 87

$4.2 \quad$ Terceira homologia de $G M_{2} \ldots \ldots \ldots \ldots$. . . . . . . . . 90

$4.3 \quad$ Terceira homologia de $G L_{3}$ de um corpo . . . . . . . . . . . 98

$4.4 \quad$ A sequência exata de Bloch-Wigner . . . . . . . . . . . 106

REFERÊNCIAS . . . . . . . . . . . . . . . . . . 111 

O estudo da sequência exata de Bloch-Wigner é justificada pelo estudo do segundo e terceiro $K$-grupos de um anel local. A sequência exata de Bloch-Wigner aparece em diferentes áreas da matemática, como a teoria dos números, a geometria hiperbólica em dimensão três, a $K$-teoria algébrica, etc.

A $K$-teoria algébrica é um ramo da álgebra que generaliza o estudo da álgebra linear, associando para cada anel com unidade $R$ uma sequência de grupos abelianos $K_{n}(R)$ chamados os $n$-ésimos $K$-grupos de $R$.

Estes grupos foram definidos por Daniel Quillen em 1970 a partir da introdução da +- construção do espaço classificante $B G L(R)$. Sabe-se que estes grupos têm um produto com estrutura anti-comutativa.

Nesta dissertação, vamos provar que se $R$ é um anel local com corpo residual infinito, então

$$
K_{2}(R) \simeq \frac{R^{\times} \otimes_{\mathbb{Z}} R^{\times}}{\left\langle a \otimes(1-a): a \in R^{\times}-\{1\}\right\rangle}
$$

Isto é uma generalização de Van der Kallen [13] do teorema de Matsumoto que dá uma presentação do segundo $K$-grupo de um corpo. Generalizando essas ideias, Milnor define novos $K$-grupos, agora chamados $K$-grupos de Milnor. Assim para qualquer anel comutativo $R$ e para todo $n \geq 0$, agora podemos definir os $K$-grupos de Milnor $K_{n}^{M}(R)$ e definir neles um produto também com estrutura anti-comutativa.

Usando o produto dos $K$-grupos de Quillen e Milnor e suas estruturas anti-comutativas, definimos de maneira indutiva um homomorfismo

$$
\tau_{n}: K_{n}^{M}(R) \rightarrow K_{n}(R)
$$

Nesta dissertação estudaremos estes homomorfismos para $0 \leq n \leq 3$. Se $R$ for um anel local com corpo residual infinito, demonstraremos que $\tau_{n}$ é um isomorfismo para $0 \leq n \leq 2$. Mas em geral este homomorfismo nem sempre é injetor ou sobrejetor. No caso $n=3$ estudaremos o grupo

$$
K_{3}^{\text {ind }}(F):=\operatorname{coker}\left(K_{3}^{M}(F) \rightarrow K_{3}(F)\right),
$$

chamado a parte indecomponível de $K_{3}(F)$, onde $F$ é um corpo infinito. 
A sequência exata de Bloch-Wigner dá uma descrição explícita da parte indecomponível do terceiro $K$-grupo de um corpo infinito $F$. Na forma original, demonstrada por Bloch e Wigner separadamente, o resultado garante a existência de uma sequência exata para um corpo algebricamente fechado $k$ de característica zero, da forma

$$
0 \rightarrow \mathbb{Q} / \mathbb{Z} \rightarrow K_{3}^{\text {ind }}(k) \rightarrow \mathfrak{p}(k) \rightarrow k^{\times} \wedge k^{\times} \rightarrow K_{2}(k) \rightarrow 0,
$$

onde $\mathfrak{p}(k)$ é o grupo pré-Bloch de $k$ que é o grupo quociente do grupo abeliano livre com base nos símbolos $[a], a \in k^{\times}-\{1\}$, pelo subgrupo gerado pelos elementos da forma

$$
[a]-[b]+\left[\frac{b}{a}\right]-\left[\frac{1-a^{-1}}{1-b^{-1}}\right]+\left[\frac{1-a}{1-b}\right],
$$

onde $a, b \in k^{\times}-\{1\}$.

Em 1991, A. A. Suslin [12] generalizou esta sequência exata sobre qualquer corpo infinito, mostrando que para qualquer corpo infinito $F$ temos a sequência de Bloch-Wigner na forma

$$
0 \rightarrow \operatorname{Tor}_{1}^{\mathbb{Z}}(\mu(F), \mu(F))^{\sim} \rightarrow K_{3}^{\text {ind }}(F) \rightarrow \mathfrak{p}(F) \rightarrow\left(F^{\times} \otimes_{\mathbb{Z}} F^{\times}\right)_{\sigma} \rightarrow K_{2}(F) \rightarrow 0,
$$

onde $\left(F^{\times} \otimes_{\mathbb{Z}} F^{\times}\right)_{\sigma}:=F^{\times} \otimes_{\mathbb{Z}} F^{\times} /\left\langle a \otimes b+b \otimes a \mid a, b \in F^{\times}\right\rangle$, e $\operatorname{Tor}_{1}^{\mathbb{Z}}(\mu(F), \mu(F))^{\sim}$ é a única extensão não trivial de $\mathbb{Z} / 2 \mathbb{Z}$ por $\operatorname{Tor}_{1}^{\mathbb{Z}}(\mu(F), \mu(F))$ se $\operatorname{char}(F) \neq 2$ e $\mu_{2^{\infty}}(F)$ é finito e

$$
\operatorname{Tor}_{1}^{\mathbb{Z}}(\mu(F), \mu(F))^{\sim}:=\operatorname{Tor}_{1}^{\mathbb{Z}}(\mu(F), \mu(F))
$$

caso contrário.

O objetivo principal desta dissertação é apresentar uma prova deste resultado obtido por Suslin, e realizamos isso com estudo da homologia do grupo linear geral.

No primeiro capítulo definiremos as principais ferramentas da álgebra homológica usadas nesta dissertação, como os complexos de cadeias, a homologia de um grupo e as sequências espectrais.

No capítulo 2 definimos e estudamos os $K$-grupos $K_{0}(R), K_{1}(R)$ e $K_{2}(R)$, quando $R$ for um anel local. Como resultado principal deste capítulo mostraremos o teorema de Van der Kallen-Matsumoto [Teorema 2.2] que prova que $K_{2}(R) \simeq K_{2}^{M}(R)$, quando $R$ for um anel local com corpo residual infinito. Para fazer isso, introduziremos e estudaremos a sequência espectral

$$
E_{p, q}^{1}=H_{q}\left(G L_{2}(R), C_{p}\left(R^{2}\right)\right) \Rightarrow H_{p+q}\left(G L_{2}(R)\right)
$$


onde $C_{p}\left(R^{2}\right)$ é o grupo abeliano livre gerado pelas $(p+1)$-uplas $\left(\left\langle v_{0}\right\rangle, \ldots\left\langle v_{p}\right\rangle\right)$ tal que para todo $i \neq j, R^{2}=\left\langle v_{i}, v_{j}\right\rangle$.

No capítulo 3, após uma breve introdução de algumas construções sobre espaços topológicos; especialmente os $C W$-complexos, os grupos de homotopia e a +- construção de um espaço; daremos as definições dos $K$-groups $K_{n}(R)$ e $K_{n}^{M}(R)$ e estudaremos seus produtos e suas estruturas anti-comutativas. Além disso, construiremos indutivamente o homomorfismo natural $\tau_{n}: K_{n}^{M}(R) \rightarrow K_{n}(R)$. Especialmente daremos uma descrição do grupo $K_{3}^{\text {ind }}(R)$ [Corolário 3.2] em termos da terceira homologia do grupo linear especial $S L(R)$, quando $R$ for um anel local com corpo residual infinito.

No capítulo 4, estudaremos primeiro a terceira homologia dos grupos $G L_{2}$ e $G L_{3}$, e usando os resultados obtidos nesta parte mostraremos o teorema principal desta dissertação que garante a existência da sequência exata de Bloch-Wigner [Teorema 4.4] para o corpo infinito. 



\section{1}

\section{HOMOLOGIA DE GRUPOS}

Nesse capítulo falamos sobre à álgebra homológica, conceitos fundamentais e toda a nomenclatura necessária para os capítulos subsequentes. Iniciamos com a definição de homologia de complexos e suas propriedades. Depois partimos para algumas noções como a homologia de grupos e damos alguns exemplos. Apresentaremos também as noções mais importantes das sequências espectrais, que é a ferramenta algébrica de maior relevância nesse trabalho, e finalmente alguns resultados da homologia dos grupos lineares.

\subsection{Complexos de cadeias}

Seja $R$ um anel (não necessariamente comutativo) com unidade. Um complexo de cadeias de $R$-módulos (à direita ou à esquerda) $\left(C_{\bullet}, d_{\bullet}\right)$ é uma sequência de $R$-módulos (à direita ou à esquerda) $C_{\bullet}=\left\{C_{n}\right\}_{n \in \mathbb{Z}}$ e uma família de $R$-homomorfismos $d_{\bullet}=\left\{d_{n}: C_{n} \rightarrow C_{n-1}\right\}_{n \in \mathbb{Z}}$, tais que a composição de quaisquer dois morfismos consecutivos é zero, isto é, para cada $n$

$$
d_{n} \circ d_{n+1}=0 .
$$

É fácil verificar que $\operatorname{Im} d_{n+1} \subseteq \operatorname{Ker} d_{n}$. Denotamos o complexo de cadeias $\left(C_{\bullet}, d_{\bullet}\right)$ também por

$$
C_{\bullet}: \cdots \longrightarrow C_{n+1} \stackrel{d_{n+1}}{\longrightarrow} C_{n} \stackrel{d_{n}}{\longrightarrow} C_{n-1} \longrightarrow \cdots \text {. }
$$

Definimos o $n$-ésimo grupo de homologia do complexo $\left(C_{\bullet}, d_{\bullet}\right)$ como o quociente

$$
H_{n}\left(C_{\bullet}\right):=\frac{\operatorname{Ker} d_{n}}{\operatorname{Im} d_{n+1}} .
$$

Se $H_{n}\left(C_{\bullet}\right)=0$ para todo $n$, então dizemos que o complexo $\left(C_{\bullet}, d_{\bullet}\right)$ é exato.

EXEMPLO 1.1. Considere $R$ e $S$ anéis com unidade.

1) Para um $R$-módulo $M$, definimos o complexo $\left(M_{\bullet}, d_{\bullet}\right)$ como sendo $M_{n}=0$ para todo $n \neq 0$ e $M_{0}=M$. Definimos também os $R$-homomorfismos do complexo como sendo $d_{n}=0$ 
para todo $n$, e denotamos o complexo $\left(M_{\bullet}, d_{\bullet}\right)$ como

$$
M_{\bullet}: \cdots \longrightarrow 0 \longrightarrow 0 \longrightarrow M \longrightarrow 0 \longrightarrow 0 \longrightarrow \cdots \text {. }
$$

Em particular, é usual considerar o complexo de cadeia nulo como $0:=\left(0_{\bullet}, 0_{\bullet}\right)$.

2) Seja $\left(C_{\bullet}, d_{\bullet}\right)$ um complexo de cadeias de $R$-módulos e seja $F:{ }_{R} \operatorname{Mod} \rightarrow{ }_{S} \operatorname{Mod}$ um funtor da categoria de $R$-módulos na categoria de $S$-módulos. Então $\left(F\left(C_{\bullet}\right), F\left(d_{\bullet}\right)\right)$ definido como

$$
F\left(C_{\bullet}\right)_{n}:=F\left(C_{n}\right), \quad F\left(d_{\bullet}\right)_{n}:=F\left(d_{n}\right): F\left(C_{n}\right) \rightarrow F\left(C_{n-1}\right),
$$

é um complexo de cadeias de $S$-módulos.

Um complexo de cadeias $\left(C_{\bullet}, d_{\bullet}\right)$ é dito não negativo, se $C_{n}=0$ para todo $n<0$ e escreve-se

$$
C_{\bullet}: \cdots \longrightarrow C_{n+1} \stackrel{d_{n+1}}{\longrightarrow} C_{n} \stackrel{d_{n}}{\longrightarrow} \cdots \longrightarrow C_{1} \stackrel{d_{1}}{\longrightarrow} C_{0} \longrightarrow 0 .
$$

Sejam $\left(C_{\bullet}, d_{\bullet}\right)$ e $\left(C_{\bullet}^{\prime}, d_{\bullet}^{\prime}\right)$ complexos de cadeias de $R$-módulos. Um morfismo de complexos $\alpha_{\bullet}:\left(C_{\bullet}, d_{\bullet}\right) \rightarrow\left(C_{\bullet}^{\prime}, d_{\bullet}^{\prime}\right)$ é uma família de $R$-homomorfismos $\left\{\alpha_{n}: C_{n} \rightarrow C_{n}^{\prime}\right\}_{n \in \mathbb{Z}}$ tais que $\alpha_{n-1} \circ$ $d_{n}=d_{n}^{\prime} \circ \alpha_{n}$, isto é para todo $n \in \mathbb{Z}$ o seguinte diagrama comuta

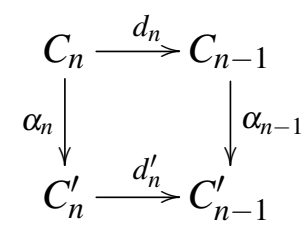

Assim, um morfismo de complexos de cadeias $\alpha_{\bullet}:\left(C_{\bullet}, d_{\bullet}\right) \rightarrow\left(C_{\bullet}^{\prime}, d_{\bullet}^{\prime}\right)$ induz para cada $n \in \mathbb{Z}$ um homomorfismo

$$
\alpha_{*}: H_{n}\left(C_{\bullet}\right) \rightarrow H_{n}\left(C_{\bullet}^{\prime}\right), \quad x+\operatorname{Im} d_{n+1} \longmapsto \alpha_{n}(x)+\operatorname{Im} d_{n+1}^{\prime}, \quad x \in \operatorname{Ker} d_{n} .
$$

Se $\beta_{\bullet}:\left(C_{\bullet}^{\prime}, d_{\bullet}^{\prime}\right) \rightarrow\left(C_{\bullet}^{\prime \prime}, d_{\bullet}^{\prime \prime}\right)$ é outro morfismo de complexos, então $(\beta \circ \alpha)_{*}=\beta_{*} \circ \alpha_{*}$. Em particular o morfismo identidade $i d_{C_{\bullet}}:\left(C_{\bullet}, d_{\bullet}\right) \rightarrow\left(C_{\bullet}, d_{\bullet}\right)$ induz a identidade nos grupos de homologia

$$
i d_{*}=i d_{H_{n}\left(C_{\bullet}\right)}: H_{n}\left(C_{\bullet}\right) \rightarrow H_{n}\left(C_{\bullet}\right) .
$$

Considere agora $\left(C_{\bullet}, d_{\bullet}\right),\left(C_{\bullet}^{\prime}, d_{\bullet}^{\prime}\right)$ e $\left(C_{\bullet}^{\prime \prime}, d_{\bullet}^{\prime \prime}\right)$ complexos de cadeias de $R$-módulos. Uma sequência curta de complexos é formada por morfismos de complexos de cadeias

$$
0 \longrightarrow C_{\bullet}^{\prime} \stackrel{\alpha_{\bullet}}{\longrightarrow} C_{\bullet} \stackrel{\beta_{\bullet}}{\longrightarrow} C_{\bullet}^{\prime \prime} \longrightarrow 0
$$

tais que $\beta_{n} \circ \alpha_{n}=0$ para todo $n \in \mathbb{Z}$. Esta sequência curta é dita exata se para todo $n$ a sequência curta de $R$-módulos

$$
0 \longrightarrow C_{n}^{\prime} \stackrel{\alpha_{n}}{\longrightarrow} C_{n} \stackrel{\beta_{n}}{\longrightarrow} C_{n}^{\prime \prime} \longrightarrow 0
$$

é exata. Agora, estabeleceremos a existência da sequência exata longa de homologia associada a uma sequência exata curta de complexos de cadeias. 
TEOREMA 1.1. Seja $0 \longrightarrow C_{\bullet}^{\prime} \stackrel{\alpha_{\bullet}}{\longrightarrow} C_{\bullet} \stackrel{\beta_{\bullet}}{\longrightarrow} C_{\bullet}^{\prime \prime} \longrightarrow 0$ uma sequência exata curta de complexos de cadeias de R-módulos. Então existe uma sequência exata longa de grupos de homologia

$$
\ldots \stackrel{\delta}{\rightarrow} H_{n+1}\left(C_{\bullet}^{\prime}\right) \stackrel{\alpha_{*}}{\rightarrow} H_{n+1}\left(C_{\bullet}\right) \stackrel{\beta_{*}}{\rightarrow} H_{n+1}\left(C_{\bullet}^{\prime \prime}\right) \stackrel{\delta}{\rightarrow} H_{n}\left(C_{\bullet}^{\prime}\right) \stackrel{\alpha_{*}}{\rightarrow} H_{n}\left(C_{\bullet}\right) \stackrel{\beta_{*}}{\rightarrow} H_{n}\left(C_{\bullet}^{\prime \prime}\right) \rightarrow \cdots
$$

Os homomorfismos $\delta$ são chamados homomorfismos conectantes ou de fronteira. A sequência longa anterior é natural, no sentido que se o seguinte diagrama de sequências exatas curtas de complexos de cadeias de R-módulos é comutativo

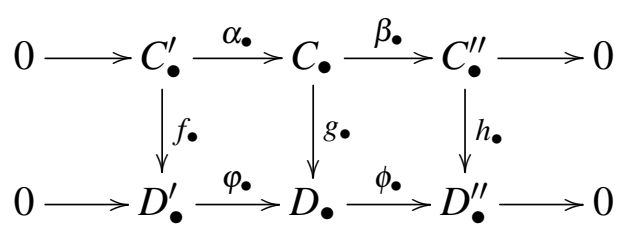

então o seguinte diagrama é comutativo

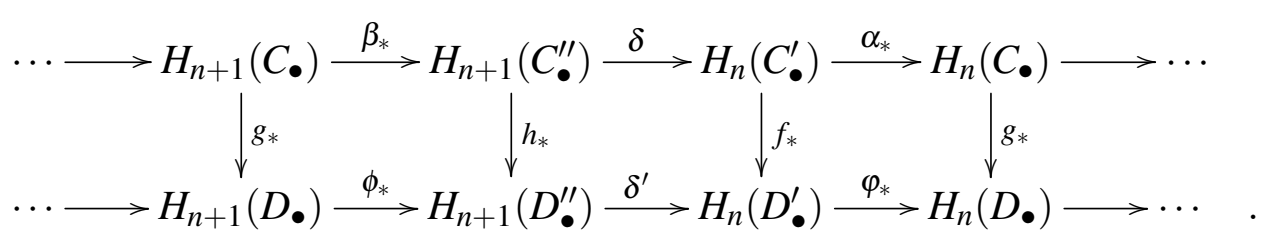

Demonstração. Veja-se [14, Teorema 4.2.4].

Sejam $\alpha_{\bullet}, \beta_{\bullet}:\left(C_{\bullet}, d_{\bullet}\right) \rightarrow\left(C_{\bullet}^{\prime}, d_{\bullet}^{\prime}\right)$ morfismos de complexos de cadeias de $R$-módulos. Dizemos que $\alpha_{\bullet}$ é homotópica (algebricamente) a $\beta_{\bullet}$ se para todo $n \in \mathbb{Z}$ existe um homomorfismo $s_{n}: C_{n} \rightarrow C_{n+1}^{\prime}$ tal que

$$
\alpha_{n}-\beta_{n}=d_{n+1}^{\prime} \circ s_{n}+s_{n-1} \circ d_{n} .
$$

Dizemos que $\alpha_{\bullet}$ é homotopicamente nula se $\alpha_{\bullet}$ é homotópica ao morfismo nulo

$$
0:=0_{\bullet}:\left(C_{\bullet}, d_{\bullet}\right) \rightarrow\left(C_{\bullet}^{\prime}, d_{\bullet}^{\prime}\right), \quad x \longmapsto 0 .
$$

EXEMPlo 1.2. Seja $\left(C_{\bullet}, d_{\bullet}\right)$ um complexo de cadeias. Se a identidade $i d_{C_{\bullet}}:\left(C_{\bullet}, d_{\bullet}\right) \rightarrow\left(C_{\bullet}, d_{\bullet}\right)$ é homotopicamente nula, então para cada $n \in \mathbb{Z}$ existe um homomorfismo $s_{n}: C_{n} \rightarrow C_{n+1}$ tal que para todo $x \in \operatorname{Ker} d_{n}$

$$
x=i d_{C_{n}}(x)=d_{n+1}\left(s_{n}(x)\right)+s_{n-1}\left(d_{n}(x)\right)=d_{n+1}\left(s_{n}(x)\right) \in \operatorname{Im} d_{n+1} .
$$

Portanto, o complexo $\left(C_{\bullet}, d_{\bullet}\right)$ é exato.

A seguinte proposição estabelece que dois morfismos de complexos homotópicos induzem o mesmo homomorfismo entre os grupos de homologia.

Proposição 1.1. Sejam $\alpha_{\bullet}, \beta_{\bullet}:\left(C_{\bullet}, d_{\bullet}\right) \rightarrow\left(C_{\bullet}^{\prime}, d_{\bullet}^{\prime}\right)$ morfismos de complexos de cadeias de $R$-módulos. Se $\alpha_{\bullet}$ é homotópica a $\beta_{\bullet}$, então

$$
\alpha_{*}=\beta_{*}: H_{n}\left(C_{\bullet}\right) \rightarrow H_{n}\left(C_{\bullet}^{\prime}\right) .
$$


Demonstração. Veja-se [14, Teorema 4.3.3].

Exemplo 1.3. Sejam $\alpha_{\bullet}, \beta_{\bullet}:\left(C_{\bullet}, d_{\bullet}\right) \rightarrow\left(C_{\bullet}^{\prime}, d_{\bullet}^{\prime}\right)$ morfismos de complexos de cadeias de $R$ módulos e seja $F:{ }_{R} \operatorname{Mod} \rightarrow{ }_{S} \operatorname{Mod}$ um funtor. Então

$$
F\left(\alpha_{\bullet}\right), F\left(\beta_{\bullet}\right):\left(F\left(C_{\bullet}\right), F\left(d_{\bullet}\right)\right) \rightarrow\left(F\left(C_{\bullet}^{\prime}\right), F\left(d_{\bullet}^{\prime}\right)\right),
$$

são morfismos de complexos de cadeias de $S$-módulos. Se $\alpha_{\bullet}$ é homotópica a $\beta_{\bullet}$ e $F$ é um funtor aditivo, isto é para todo $R$-módulo $M$ e $N$, a aplicação

$$
F: \operatorname{Hom}_{R}(M, N) \rightarrow \operatorname{Hom}_{S}(F(M), F(N)), \quad f \rightarrow F(f),
$$

é um homomorfismo de grupos abelianos, então $F\left(\alpha_{\bullet}\right)$ é homotópica a $F\left(\beta_{\bullet}\right)$.

O seguinte lema permite estender uma certa família de $R$-homomorfismos para um morfismo de complexo de cadeias de forma única, a menos de homotopia algébrica.

LEMA 1.1. Sejam $\left(C_{\bullet}, d_{\bullet}\right) e\left(C_{\bullet}^{\prime}, d_{\bullet}^{\prime}\right)$ complexos de cadeias de $R$-módulos. Seja $r \in \mathbb{Z}$ e considere $\left\{\alpha_{n}: C_{n} \rightarrow C_{n}^{\prime}\right\}_{n \leq r}$ uma família de R-homomorfismos tais que $d_{n}^{\prime} \circ \alpha_{n}=\alpha_{n-1} \circ d_{n}$ para todo $n \leq r$. Se $C_{n}$ é um $R$-módulo projetivo para todo $n>r$ e $H_{n}\left(C_{\bullet}^{\prime}\right)=0$ para todo $n \geq r$, então a família de R-homomorfismos $\alpha_{n}$ é estendida para um morfismo de complexos de cadeias de $R$-módulos $\alpha_{\bullet}:\left(C_{\bullet}, d_{\bullet}\right) \rightarrow\left(C_{\bullet}^{\prime}, d_{\bullet}^{\prime}\right)$ e $\alpha_{\bullet}$ é única a menos de homotopia, isto é qualquer duas extensões da família $\left\{\alpha_{n}\right\}_{n \leq r}$ são homotópicas.

Demonstração. Veja-se [1, Lema 7.4, Ch. I].

Agora estudaremos complexos de cadeias associados a um $R$-módulo dado. Seja $M$ um $R$-módulo. Uma resolução de $M$ é uma sequência exata da forma

$$
C_{\bullet} \stackrel{\varepsilon}{\rightarrow} M: \quad \cdots \longrightarrow C_{n+1} \stackrel{d_{n+1}}{\longrightarrow} C_{n} \stackrel{d_{n}}{\rightarrow} C_{n-1} \longrightarrow \cdots \longrightarrow C_{2} \stackrel{d_{2}}{\longrightarrow} C_{1} \stackrel{d_{1}}{\rightarrow} C_{0} \stackrel{\varepsilon}{\rightarrow} M \longrightarrow 0 .
$$

Se para todo $n \geq 0, C_{n}$ é um $R$-módulo projetivo, então $C_{\bullet} \rightarrow M$ é dita uma resolução projetiva de $M$ sobre $R$. Se para todo $n \geq 0, C_{n}$ é um $R$-módulo livre (ou plano), então $C_{\bullet} \rightarrow M$ é dita uma resolução livre (ou plana) de $M$ sobre $R$.

O seguinte lema garante a existência de resoluções projetivas para cada $R$-módulo e o teorema a seguir as relaciona de forma única, a menos de homotopia.

LEMA 1.2. Todo R-módulo $M$ tem uma resolução livre, e logo também tem uma resolução projetiva e uma resolução plana.

Demonstração. Veja-se [15, Lema 2.2.5].

TEOREMA 1.2. Sejam $F_{\bullet} \stackrel{\varepsilon}{\rightarrow} M$ e $F_{\bullet}^{\prime} \stackrel{\varepsilon^{\prime}}{\rightarrow} M$ resolucões projetivas do $R$-módulo $M$. Então existe um morfismo de complexos de cadeias $\alpha_{\bullet}:\left(F_{\bullet}, d_{\bullet}\right) \rightarrow\left(F_{\bullet}^{\prime}, d_{\bullet}^{\prime}\right)$ tal que $\varepsilon^{\prime} \circ \alpha_{\bullet}=\varepsilon$. O morfismo de complexos $\alpha_{\bullet}$ é único a menos de homotopia. 
Demonstração. Veja-se [1, Teorema 7.5, Ch. I].

Um morfismo de complexos de cadeias $\alpha_{\bullet}:\left(C_{\bullet}, d_{\bullet}\right) \rightarrow\left(C_{\bullet}^{\prime}, d_{\bullet}^{\prime}\right)$ é dito uma equivalência homotópica se existe um morfismo de complexos $\beta_{\bullet}:\left(C_{\bullet}^{\prime}, d_{\bullet}^{\prime}\right) \rightarrow\left(C_{\bullet}, d_{\bullet}\right)$ tal que

i) $\beta_{\bullet} \circ \alpha_{\bullet}$ é homotópica a $i d_{C_{\bullet}}:\left(C_{\bullet}, d_{\bullet}\right) \rightarrow\left(C_{\bullet}, d_{\bullet}\right)$,

ii) $\alpha_{\bullet} \circ \beta_{\bullet}$ é homotópica a $i d_{C_{\bullet}^{\prime}}:\left(C_{\bullet}^{\prime}, d_{\bullet}^{\prime}\right) \rightarrow\left(C_{\bullet}^{\prime}, d_{\bullet}^{\prime}\right)$.

Por outro lado, se $\alpha_{*}: H_{n}\left(C_{\bullet}\right) \rightarrow H_{n}\left(C_{\bullet}^{\prime}\right)$ é um isomorfismo para todo $n$, então $\alpha_{\bullet}$ é dita uma equivalência fraca. É fácil ver que toda equivalência homotópica é uma equivalência fraca.

TEOREMA 1.3. Sejam $F_{\bullet} \rightarrow M$ e $F_{\bullet}^{\prime} \rightarrow M$ resoluções projetivas (à direita) do $R-$ módulo $M$. Então existe um morfismo de complexos $\alpha_{\bullet}:\left(F_{\bullet}, d_{\bullet}\right) \rightarrow\left(F_{\bullet}^{\prime}, d_{\bullet}^{\prime}\right)$ tal que para qualquer $R$-módulo à esquerda $\mathrm{N}$ o morfismo

$$
\alpha_{\bullet} \otimes i d_{M}: F_{\bullet} \otimes_{R} N \rightarrow F_{\bullet}^{\prime} \otimes_{R} N
$$

é uma equivalência fraca. Em particular, $H_{n}\left(F_{\bullet} \otimes_{R} N\right) \cong H_{n}\left(F_{\bullet}^{\prime} \otimes_{R} N\right)$ para todo $n \geq 0$.

Demonstração. Veja-se [1, Teorema 8.6, Ch. I].

Seja $F_{\bullet} \rightarrow M$ uma resolução projetiva do $R$-módulo à direita $M$. Definimos para qualquer $R$ módulo à esquerda $N$ e para todo $n \geq 0, \mathrm{o} R-$ módulo

$$
\operatorname{Tor}_{n}^{R}(M, N):=H_{n}\left(F_{\bullet} \otimes_{R} N\right)
$$

Pelo Teorema (1.3), o $R$-módulo $\operatorname{Tor}_{n}^{R}(M, N)$ é independente da escolha da resolucão projetiva de $M$. Os seguintes exemplos mostram as propriedades mais úteis da definição anterior.

EXEMPLO 1.4. Se $M$ e $N$ são $R$-módulos (à direita e à esquerda respetivamente), então é fácil ver que $\operatorname{Tor}_{0}^{R}(M, N) \cong M \otimes_{R} N$.

EXEMPLO 1.5. Seja $M$ um $R$-módulo à direita e seja $\left\{N_{i}\right\}_{i \in I}$ uma família de $R$-módulos à esquerda. Então para todo $n \geq 0$

$$
\operatorname{Tor}_{n}^{R}\left(M, \bigoplus_{i \in I} N_{i}\right) \cong \bigoplus_{i \in I} \operatorname{Tor}_{n}^{R}\left(M, N_{i}\right) .
$$

Mais geralmente, se $\left\{N_{i}\right\}_{i \in I}$ é uma família de $R$-módulos à esquerda tais que $I$ é um sistema direto, então para qualquer $R$-módulo à direita $M$ e para todo $n \geq 0$ temos

$$
\operatorname{Tor}_{n}^{R}\left(M, \underset{\lim }{\longrightarrow} N_{i}\right) \cong \underset{\lim }{\longrightarrow} \operatorname{Tor}_{n}^{R}\left(M, N_{i}\right)
$$

EXEMPlo 1.6. Seja $A$ um grupo abeliano. Definimos o subgrupo de torção de $A$ como sendo

$$
t A:=\{a \in A: \text { existe } n>0 \text { tal que } n a=0\} .
$$


Dizemos que $A$ é um grupo de torção se $A=t A$ e dizemos que $A$ é livre de torção se $t A=0$. Não é difícil verificar que $t(A / t A)=0, \log 0 A / t A$ é livre de torção.

Sejam $A$ e $B$ grupos abelianos. Dado que todo grupo abeliano é imagem homomorfica de um grupo abeliano livre obtemos uma resolução projetiva $F_{\bullet} \rightarrow A$ tal que $F_{n}=0$ para todo $n \geq 2$, então $\operatorname{Tor}_{n}^{\mathbb{Z}}(A, B)=0$ para $n \geq 2$. Além disso se $A$ e $B$ são grupos abelianos finitamente gerados $\operatorname{Tor}_{1}^{\mathbb{Z}}(A, B) \cong(t A) \otimes_{\mathbb{Z}}(t B)$ é um grupo de torção (veja-se [14, Corolário 6.3.16]).

De forma análoga ao caso dos grupos de homologia de uma sequência exata de complexos, estabeleceremos agora a existência de sequências exatas longas dos $R$-módulos $\operatorname{Tor}_{n}^{R}(-,-)$ associadas respetivamente a sequências exatas curtas de $R$-módulos.

TEOREMA 1.4. Seja $0 \longrightarrow N^{\prime} \stackrel{\alpha}{\longrightarrow} N \stackrel{\beta}{\longrightarrow} N^{\prime \prime} \longrightarrow 0$ uma sequência exata curta de $R$-módulos. Então para qualquer $R$-módulo $M$, existe uma sequência exata longa

$$
\cdots \rightarrow \operatorname{Tor}_{n+1}^{R}\left(M, N^{\prime \prime}\right) \stackrel{\delta}{\longrightarrow} \operatorname{Tor}_{n}^{R}\left(M, N^{\prime}\right) \stackrel{\alpha_{*}}{\longrightarrow} \operatorname{Tor}_{n}^{R}(M, N) \stackrel{\beta_{*}}{\longrightarrow} \operatorname{Tor}_{n}^{R}\left(M, N^{\prime \prime}\right) \stackrel{\delta}{\longrightarrow} \operatorname{Tor}_{n-1}^{R}\left(M, N^{\prime}\right) \rightarrow \cdots .
$$

Seja $0 \longrightarrow M^{\prime} \stackrel{\alpha^{\prime}}{\longrightarrow} M \stackrel{\beta^{\prime}}{\longrightarrow} M^{\prime \prime} \longrightarrow 0$ uma sequência exata curta de R-módulos. Então para qualquer $R$-módulo $N$, existe uma sequência exata longa

$$
\cdots \rightarrow \operatorname{Tor}_{n+1}^{R}\left(M^{\prime \prime}, N\right) \stackrel{\delta}{\longrightarrow} \operatorname{Tor}_{n}^{R}\left(M^{\prime}, N\right) \stackrel{\alpha_{*}^{\prime}}{\longrightarrow} \operatorname{Tor}_{n}^{R}(M, N) \stackrel{\beta_{*}^{\prime}}{\longrightarrow} \operatorname{Tor}_{n}^{R}\left(M^{\prime \prime}, N\right) \stackrel{\delta}{\longrightarrow} \operatorname{Tor}_{n-1}^{R}\left(M^{\prime}, N\right) \rightarrow \cdots .
$$

Demonstração. Veja-se [9, Teorema 6.21].

Com uso destas sequências exatas longas obtemos a seguinte propriedade que permite estudar melhor o grupo de torsão $\operatorname{Tor}_{1}^{\mathbb{Z}}(A, B)$ quando $A$ e $B$ são grupos abelianos.

LEMA 1.3. Se A e B são grupos abelianos, então $\operatorname{Tor}_{1}^{\mathbb{Z}}(A, B) \cong \operatorname{Tor}_{1}^{\mathbb{Z}}(t A, t B)$.

Demonstração. Veja-se [4, Capítulo 5, Seção 6].

Os seguintes teoremas ao final desta seção serão muito importantes nas seguintes seções, assim como nos próximos capítulos.

TEOREMA 1.5 (Fórmula de Künneth). Seja $\left(P_{\bullet}, d_{\bullet}\right)$ um complexo de cadeias de R-módulos planos tais que para todo $n, d_{n}\left(P_{n}\right) \subset P_{n-1}$ é um $R$-módulo plano. Então para todo $n$ e para todo $R$-módulo $M$, existe uma sequência exata

$$
0 \longrightarrow H_{n}\left(P_{\bullet}\right) \otimes_{R} M \longrightarrow H_{n}\left(P_{\bullet} \otimes_{R} M\right) \longrightarrow \operatorname{Tor}_{1}^{R}\left(H_{n-1}\left(P_{\bullet}\right), M\right) \longrightarrow 0 .
$$

Demonstração. Veja-se [15, Teorema 3.6.1].

TEOrEma 1.6 (Teorema dos Coeficientes Universais). Seja (P• P $\left._{\bullet}\right)$ um complexo de cadeias de $\mathbb{Z}$-módulos livres. Então para cada n e cada $\mathbb{Z}$-módulo $M$, a fórmula de Künneth cinde e obtemos uma decomposição (não canônica)

$$
H_{n}\left(P_{\bullet} \otimes M\right) \cong\left(H_{n}\left(P_{\bullet}\right) \otimes_{\mathbb{Z}} M\right) \oplus \operatorname{Tor}_{1}^{\mathbb{Z}}\left(H_{n-1}\left(P_{\bullet}\right), M\right) .
$$


Demonstração. Veja-se [15, Teorema 3.6.2].

Sejam $\left(C_{\bullet}, d_{\bullet}\right)$ e $\left(C_{\bullet}^{\prime}, d_{\bullet}^{\prime}\right)$ complexos de $R$-módulos e considere os $R$-módulos

$$
\left(C \bullet \otimes C_{\bullet}^{\prime}\right)_{n}:=\bigoplus_{p+q=n} C_{p} \otimes C_{q}^{\prime}
$$

e os $R$-homomorfismos

$$
\partial_{n}:\left(C_{\bullet} \otimes C_{\bullet}^{\prime}\right)_{n} \longrightarrow\left(C_{\bullet} \otimes C_{\bullet}^{\prime}\right)_{n-1}, \quad x \otimes y \longmapsto d_{p}(x) \otimes y+(-1)^{p} x \otimes d_{q}^{\prime}(y),
$$

onde $p+q=n, x \in C_{p}, y \in C_{q}^{\prime}$. Claramente $\partial_{n-1} \circ \partial_{n}(x \otimes y)=0$ e portanto $\left(C_{\bullet} \otimes C_{\bullet}^{\prime}, \partial_{\bullet}\right)$ é um complexo de $R$-módulos, chamado complexo produto de $C_{\bullet}$ e $C_{\bullet}^{\prime}$. Além disso, se $d_{p}(x)=0$ e $d_{q}^{\prime}(y)=0$, então $\partial_{n}(x \otimes y)=d_{p}(x) \otimes y+(-1)^{p} x \otimes d_{q}^{\prime}(y)=0$. Assim temos um homomorfismo bem definido

$H_{p}\left(C_{\bullet}\right) \otimes H_{q}\left(C_{\bullet}^{\prime}\right) \longrightarrow H_{p+q}\left(C_{\bullet} \otimes C_{\bullet}^{\prime}\right), \quad\left(x+\operatorname{Im} d_{p+1}\right) \otimes\left(y+\operatorname{Im} d_{q+1}^{\prime}\right) \longmapsto x \otimes y+\operatorname{Im} \partial_{n+1}$,

onde $x \in \operatorname{Ker} d_{p}, y \in \operatorname{Ker} d_{q}^{\prime}$.

TEOREMA 1.7. Sejam $\left(P_{\bullet}, d_{\bullet}\right)$ e $\left(Q_{\bullet}, d_{\bullet}^{\prime}\right)$ complexos de cadeias de R-módulos (à direita e à esquerda respetivamente). Se $P_{n}$ e $d_{n}\left(P_{n}\right)$ são $R$-módulos planos para todo $n$, então existe uma sequência exata

$$
0 \longrightarrow \bigoplus_{p+q=n} H_{p}\left(P_{\bullet}\right) \otimes_{R} H_{q}\left(Q_{\bullet}\right) \longrightarrow H_{n}\left(P_{\bullet} \otimes Q_{\bullet}\right) \longrightarrow \bigoplus_{p^{\prime}+q^{\prime}=n-1} \operatorname{Tor}_{1}^{R}\left(H_{p^{\prime}}\left(P_{\bullet}\right), H_{q^{\prime}}\left(Q_{\bullet}\right)\right) \longrightarrow 0
$$

Demonstração. Veja-se [15, Teorema 3.6.3].

COROLÁRIO 1.1 (Fórmula de Künneth para complexos). Sejam $\left(P_{\bullet}, d_{\bullet}\right) e\left(Q_{\bullet}, d_{\bullet}^{\prime}\right)$ complexos de cadeias de $\mathbb{Z}-$ módulos com $P_{n}$ um $\mathbb{Z}$-módulo livre para cada $n$. Então obtemos a decomposição (não canônica)

$$
H_{n}\left(P_{\bullet} \otimes Q_{\bullet}\right) \cong\left(\bigoplus_{p+q=n} H_{p}\left(P_{\bullet}\right) \otimes_{\mathbb{Z}} H_{q}\left(Q_{\bullet}\right)\right) \bigoplus\left(\bigoplus_{p^{\prime}+q^{\prime}=n-1} \operatorname{Tor}_{1}^{\mathbb{Z}}\left(H_{p^{\prime}}\left(P_{\bullet}\right), H_{q^{\prime}}\left(Q_{\bullet}\right)\right)\right)
$$

Demonstração. O resultado segue do Teorema (1.7) e do fato que os $\mathbb{Z}$-submódulos de $\mathbb{Z}$-módulos livres são livres.

\subsection{Homologia de Grupos}

Seja $G$ um grupo (multiplicativo). O anel de grupo de $G$, denotado $\mathbb{Z} G$, é o $\mathbb{Z}$-módulo livre gerado pelos elementos de $G$

$$
\mathbb{Z} G:=\bigoplus_{g \in G} \mathbb{Z} \cdot g
$$


junto com a multiplicação

$$
\begin{aligned}
\mathbb{Z} G \times \mathbb{Z} G & \longrightarrow \mathbb{Z} G . \\
\left(\sum n_{i} g_{i}, \sum n_{j} g_{j}\right) & \longmapsto \sum n_{i} n_{j} g_{i} g_{j} .
\end{aligned}
$$

Definimos um $G$-módulo à esquerda como sendo um grupo abeliano $A$ junto com uma ação de $G$ sobre $A$ tal que

$$
g \cdot(a+b)=g \cdot a+g \cdot b .
$$

Se $A$ é um $G$-módulo, então temos um homomorfismo de grupos

$$
\sigma: G \longrightarrow \text { Aut } A, \quad g \longmapsto \sigma_{g} \in \text { End } A,
$$

onde $\sigma_{g}: A \rightarrow A, g \mapsto g \cdot a$. Portanto existe uma única extensão $\bar{\sigma}: \mathbb{Z} G \rightarrow$ End $A, \sigma\left(\sum n_{g} g\right)=$ $\sum n_{g} \sigma_{g}$ isto é, $A$ é um $\mathbb{Z} G$-módulo. É claro que todo $\mathbb{Z} G$-módulo é um $G$-módulo. Assim, $\mathbb{Z} G$-módulos e $G$-módulos significaram o mesmo. Se $A$ é um $G$-módulo à esquerda, podemos definir uma estrutura de $G$-módulo à direita fazendo uso da ação: $a \cdot g:=g^{-1} \cdot a$. Definimos um $G$-módulo trivial como sendo um grupo abeliano $A$, onde $G$ age trivialmente sobre $A$, isto é para todo $g \in G$ e para todo $a \in A, g \cdot a=a$. Para a maior parte dos resultados que vamos mostrar nesse trabalho, consideraremos sempre $\mathbb{Z}$ como um $G$-módulo trivial.

Para qualquer grupo $G$, definimos o homomorfismo de augmentação (de $G$ ) como sendo o homomorfismo de anéis

$$
\varepsilon: \mathbb{Z} G \longrightarrow \mathbb{Z}, \quad \sum n_{i} g_{i} \longmapsto \sum n_{i}
$$

Assim também definimos o ideal aumentado como sendo $I_{G}:=\operatorname{ker} \varepsilon$. Note que $\{g-1: g \in G\}$ é uma $\mathbb{Z}$-base de $I_{G}$. Por outro lado, definimos o grupo de coinvariantes do $G$-módulo $M$ como sendo o grupo abeliano

$$
M_{G}:=M / I_{G} M .
$$

Na verdade, $I_{G} M$ é o submódulo de $M$ gerado pelos elementos da forma $g \cdot m-m$, onde $g \in G$, $m \in M$. Note que $M_{G}$ é o maior $G$-módulo quociente de $M$ onde $G$ age trivialmente. É fácil verificar que

$$
M_{G} \cong \mathbb{Z} \otimes_{\mathbb{Z} G} M \cong M \otimes_{\mathbb{Z} G} \mathbb{Z}
$$

Por exemplo, $\mathbb{Z} \otimes_{\mathbb{Z} G} M \cong \frac{\mathbb{Z} G}{I_{G}} \otimes_{\mathbb{Z} G} M \cong M / I_{G} M=M_{G}$.

Um $G$-homomorfismo $f: M \rightarrow M^{\prime}$ de $G$-módulos é um homomorfismo de grupos abelianos que respeita as ações, isto é $f$ é um homomorfismo de grupos abelianos tal que para todo $g \in G$ e para todo $m \in M$,

$$
f(g \cdot m)=g \cdot f(m) .
$$

Definimos o funtor $-{ }_{G}$ que leva a categoria dos $G$-módulos para a categoria dos grupos abelianos

$$
\begin{aligned}
{ }_{G}:{ }_{G} \operatorname{Mod} & \longrightarrow \mathfrak{A} \mathfrak{b}, \\
M & \longmapsto M_{G}, \\
\left(f: M \rightarrow M^{\prime}\right) & \longmapsto f_{G}: M_{G} \rightarrow M_{G}^{\prime},
\end{aligned}
$$


onde $f_{G}(\bar{m})=\overline{f(m)}$. Note que $f_{G}$ está bem definida pois $f(g \cdot m-m)=g \cdot f(m)-f(m)$.

Com estas ferramentas definimos a homologia de un grupo. Considere $G$ um grupo e $M$ um $G$-módulo. Para $n \geq 0$, definimos o $n$-ésimo grupo de homologia de $G$ com coeficientes em $M$ como

$$
H_{n}(G, M):=\operatorname{Tor}_{n}^{\mathbb{Z} G}(\mathbb{Z}, M)=H_{n}\left(P_{\bullet} \otimes_{\mathbb{Z} G} M\right)=H_{n}\left(\left(P_{\bullet}\right)_{G}\right),
$$

onde $P_{\bullet} \rightarrow \mathbb{Z}$ é uma resolução projetiva de $\mathbb{Z}$ sobre $G$. Usaremos em diante a notação

$$
M \otimes_{G} N:=M \otimes_{\mathbb{Z} G} N
$$

Não é difícil verificar que $M \otimes_{G} N \cong N \otimes_{G} M$. Desta maneira, escrevemos

$$
H_{n}(G, M)=H_{n}\left(P_{\bullet} \otimes_{G} M\right)
$$

No caso quando $M=\mathbb{Z}$ dizemos que $H_{n}(G, \mathbb{Z})$ é o $n$-ésimo grupo de homologia integral de $G$ e escrevemos

$$
H_{n}(G):=H_{n}(G, \mathbb{Z})=H_{n}\left(Q \bullet \otimes_{G} \mathbb{Z}\right),
$$

onde $Q \bullet \rightarrow \mathbb{Z}$ é uma resolução projetiva de $\mathbb{Z}$ sobre $G$. Pelo Exemplo (1.4) temos que

$$
H_{0}(G, M)=\operatorname{Tor}_{0}^{\mathbb{Z} G}(\mathbb{Z}, M) \cong \mathbb{Z} \otimes_{G} M \cong M_{G}
$$

Em particular, $H_{0}(G) \cong \mathbb{Z}_{G}=\mathbb{Z}$ pois $\mathbb{Z}$ é um $G$-módulo trivial. Usando o Teorema (1.4) e a definição anterior, para cada sequência exata curta de $G$-módulos temos associada uma sequência exata longa nos grupos de homologia de $G$ com coeficientes nos $G$-módulos em questão.

TEOREMA 1.8. Seja $0 \longrightarrow M^{\prime} \stackrel{\alpha}{\longrightarrow} M \stackrel{\beta}{\longrightarrow} M^{\prime \prime} \longrightarrow 0$ uma sequência exata de G-módulos. Então existe uma sequência exata longa natural de grupos de homologia

$$
\cdots \rightarrow H_{n+1}\left(G, M^{\prime \prime}\right) \stackrel{\delta}{\longrightarrow} H_{n}\left(G, M^{\prime}\right) \stackrel{\alpha_{*}}{\longrightarrow} H_{n}(G, M) \stackrel{\beta_{*}}{\longrightarrow} H_{n}\left(G, M^{\prime \prime}\right) \stackrel{\delta}{\longrightarrow} H_{n-1}\left(G, M^{\prime}\right) \rightarrow \cdots
$$

Os seguintes exemplos que introduzimos serão usados nos próximos capítulos para simplificar os cálculos.

EXEMPLO 1.7. Seja $\left\{M_{i}\right\}_{i \in I}$ uma família de $G$-módulos. Então pelo Exemplo (1.5) temos

$$
H_{n}\left(G, \bigoplus_{i \in I} M_{i}\right)=\operatorname{Tor}_{n}^{\mathbb{Z} G}\left(\mathbb{Z}, \bigoplus_{i \in I} M_{i}\right) \cong \bigoplus_{i \in I} \operatorname{Tor}_{n}^{\mathbb{Z} G}\left(\mathbb{Z}, M_{i}\right)=\bigoplus_{i \in I} H_{n}\left(G, M_{i}\right)
$$

Proposição 1.2. Seja $\left\{G_{i}\right\}_{i \in I}$ uma família de grupos tais que I é um sistema direito e seja $G:=\lim _{\rightarrow} G_{i}$. Então para qualquer $G$-módulo $M$ e para qualquer $n \geq 0$ temos

$$
H_{n}(G, M) \cong \lim _{\rightarrow} H_{n}\left(G_{i}, M\right) .
$$

Demonstração. Veja-se [1, pág. 121]. 
Exemplo 1.8. Seja $G=\{1\}$, então um $G$-módulo é sempre trivial e portanto $M_{G}=M$. Se $P_{\bullet} \rightarrow \mathbb{Z}$ é uma resolução projetiva de $\mathbb{Z}$ sobre $G$, então $\left(P_{\bullet}\right)_{G}=P_{\bullet}$. Logo

$$
H_{n}(G, M) \cong \begin{cases}M & \text { se } n=0 \\ 0 & \text { se } n \neq 0\end{cases}
$$

Exemplo 1.9. Se $M$ é um $G$-módulo projetivo, então para todo $n \geq 1, \operatorname{Tor}_{n}^{\mathbb{Z} G}(\mathbb{Z}, M)=0$. Portanto

$$
H_{n}(G, M) \cong \begin{cases}M_{G} & \text { se } n=0 \\ 0 & \text { se } n \neq 0\end{cases}
$$

Em particular

$$
H_{n}(G, \mathbb{Z} G) \cong\left\{\begin{array}{ll}
(\mathbb{Z} G)_{G} & \text { se } n=0, \\
0 & \text { se } n \neq 0 .
\end{array}= \begin{cases}\mathbb{Z} & \text { se } n=0, \\
0 & \text { se } n \neq 0 .\end{cases}\right.
$$

Exemplo 1.10. Se $M$ é um $G$-módulo trivial, então $H_{0}(G, M)=M$. Em particular, se $M=\mathbb{Z}$, então a sequência exata $0 \longrightarrow I_{G} \longrightarrow \mathbb{Z} G \stackrel{\varepsilon}{\rightarrow} \mathbb{Z} \longrightarrow 0$ gera a sequência exata longa

$$
\cdots \longrightarrow H_{1}(G, \mathbb{Z} G) \longrightarrow H_{1}(G) \longrightarrow H_{0}\left(G, I_{G}\right) \longrightarrow H_{0}(G, \mathbb{Z} G) \longrightarrow H_{0}(G) \longrightarrow 0 .
$$

Agora pelo Exemplo (1.9)

$$
H_{1}(G, \mathbb{Z} G)=0, \quad H_{0}(G, \mathbb{Z} G)=\mathbb{Z} G \otimes_{G} \mathbb{Z}=\mathbb{Z} .
$$

Por outro lado, $H_{0}(G) \cong \mathbb{Z} \mathrm{e}$

$$
H_{0}\left(G, I_{G}\right)=I_{G} \otimes_{G} \mathbb{Z}=I_{G} \otimes_{G}\left(\frac{\mathbb{Z} G}{I_{G}}\right) \cong \frac{I_{G}}{I_{G}^{2}} .
$$

É fácil verificar que

$$
\varphi: \frac{G}{[G, G]} \rightarrow \frac{I_{G}}{I_{G}^{2}}, \quad \quad \varphi(g[G, G])=(g-1) I_{G}^{2},
$$

é um isomorfismo, onde $[G, G]$ é o subgrupo comutador de $G$. Portanto

$$
H_{0}\left(G, I_{G}\right) \cong \frac{G}{[G, G]}
$$

Desta maneira temos a sequência exata

$$
0 \longrightarrow H_{1}(G) \longrightarrow \frac{G}{[G, G]} \longrightarrow \mathbb{Z} \stackrel{\varepsilon_{*}}{\longrightarrow} \mathbb{Z} \longrightarrow 0
$$

Mas

$$
\begin{aligned}
\varepsilon_{*}: \mathbb{Z} \cong \mathbb{Z} G \otimes_{G} \mathbb{Z} & \longrightarrow \mathbb{Z} \otimes_{G} \mathbb{Z} \cong \mathbb{Z} \\
\left(n \mapsto 1 \otimes_{G} n\right) & \longmapsto\left(1 \otimes_{G} n \mapsto n\right)
\end{aligned}
$$

é o homomorfismo identidade. Portanto

$$
H_{1}(G) \cong \frac{G}{[G, G]}
$$


EXEMPLo 1.11. Seja $G$ o grupo cíclico de ordem $n$ com gerador $t$. Como $\left\{1, t, \ldots, t^{n-1}\right\}$ é uma $\mathbb{Z}$-base para $\mathbb{Z} \mathrm{G}$, temos

$$
\mathbb{Z} G \cong \frac{\mathbb{Z}[x]}{\left\langle x^{n}-1\right\rangle}
$$

Considere $N=1+t+t^{2}+\cdots+t^{n-1} \in \mathbb{Z} G$. Não é difícil verificar que

$$
\begin{aligned}
& P_{\bullet} \stackrel{\varepsilon}{\rightarrow} \mathbb{Z}: \quad \cdots \rightarrow \mathbb{Z} G \stackrel{N}{\longrightarrow} \mathbb{Z} G \quad \stackrel{t-1}{\longrightarrow} \mathbb{Z} G \stackrel{N}{\longrightarrow} \mathbb{Z} G \stackrel{t-1}{\longrightarrow} \mathbb{Z} G \stackrel{\varepsilon}{\longrightarrow} \mathbb{Z} \rightarrow 0 \\
& g \longmapsto N \cdot g \quad g \longmapsto(t-1) \cdot g
\end{aligned}
$$

é uma resolução livre de $\mathbb{Z}$ sobre $G$. Tensorizando a resolução (1.3) por $\mathbb{Z}$ sobre $\mathbb{Z} G$, obtemos a sequência

$$
P_{\bullet} \otimes_{G} \mathbb{Z}: \quad \cdots \longrightarrow \mathbb{Z} \stackrel{n}{\longrightarrow} \mathbb{Z} \stackrel{0}{\longrightarrow} \mathbb{Z} \stackrel{n}{\longrightarrow} \mathbb{Z} \stackrel{0}{\longrightarrow} \mathbb{Z} \longrightarrow 0 .
$$

Na verdade, $n: \mathbb{Z} \rightarrow \mathbb{Z}$ e $0: \mathbb{Z} \rightarrow \mathbb{Z}$ são induzidas por

$$
\begin{aligned}
& \mathbb{Z} \cong \mathbb{Z} G \otimes_{G} \mathbb{Z} \quad \stackrel{N \otimes i d_{\mathbb{Z}}}{\longrightarrow} \mathbb{Z} G \otimes_{G} \mathbb{Z} \cong \mathbb{Z}, \\
& \left(m \mapsto 1 \otimes_{G} m\right) \quad \longmapsto\left(N \otimes_{G} m \mapsto m+\cdots+m=n m\right) \\
& \mathbb{Z} \cong \mathbb{Z} G \otimes_{G} \mathbb{Z} \quad \stackrel{(t-1) \otimes i d_{\mathbb{Z}}}{\longrightarrow} \mathbb{Z} G \otimes_{G} \mathbb{Z} \cong \mathbb{Z} . \\
& \left(m \mapsto 1 \otimes_{G} m\right) \quad \longmapsto\left((t-1) \otimes_{G} m \mapsto m-m=0\right)
\end{aligned}
$$

Portanto

$$
H_{k}(G) \cong \begin{cases}\mathbb{Z} & \text { se } k=0 \\ \mathbb{Z} / n \mathbb{Z} & \text { se } k \text { é impar } \\ 0 & \text { se } k \text { é par. }\end{cases}
$$

Em geral, tensorizando a resolução (1.3) por um $G$-módulo $M$, obtemos a sequência

$$
P \bullet \otimes_{G} M: \quad \cdots \longrightarrow M \stackrel{N}{\longrightarrow} M \stackrel{(t-1)}{\longrightarrow} M \stackrel{N}{\longrightarrow} M \stackrel{(t-1)}{\longrightarrow} M \longrightarrow 0
$$

onde os homomorfismos $N: M \rightarrow M$ e $t-1: M \rightarrow M$ são induzidas por

$$
\begin{aligned}
& M \cong \mathbb{Z} G \otimes_{G} M \stackrel{N \otimes i d_{M}}{\longrightarrow} \mathbb{Z} G \otimes_{G} M \cong M, \\
&\left(m \mapsto 1 \otimes_{G} m\right) \longmapsto\left(N \otimes_{G} m \mapsto N \cdot m\right) \\
& M \cong \mathbb{Z} G \otimes_{G} M \stackrel{(t-1) \otimes i d_{M}}{\longmapsto} \mathbb{Z} G \otimes_{G} M \cong M . \\
&\left(m \mapsto 1 \otimes_{G} m\right) \longmapsto\left((t-1) \otimes_{G} m \mapsto t m-m\right)
\end{aligned}
$$

Logo

$$
H_{k}(G, M) \cong \begin{cases}M_{G} & \text { se } k=0, \\ \frac{\operatorname{Ker}(t-1)}{\operatorname{Im} N} & \text { se } k \text { é ímpar } \\ \frac{\operatorname{Ker} N}{\operatorname{Im}(t-1)} & \text { se } k \text { é par. }\end{cases}
$$


Se $M$ é um $G$-módulo e se

$$
M^{G}:=\{m \in M: g \cdot m=m \text { para todo } g \in G\}
$$

é fácil verificar que $M^{G}=\operatorname{Ker}(t-1)$. Portanto

$$
H_{k}(G, M) \cong \begin{cases}M_{G} & \text { se } k=0, \\ \frac{M^{G}}{\operatorname{Im} N} & \text { se } k \text { é ímpar } \\ \operatorname{Ker}\left(N: M_{G} \rightarrow M^{G}\right) & \text { se } k \text { é par. }\end{cases}
$$

EXEMPLO 1.12. Seja $G$ o grupo cíclico infinito com gerador $t$. O conjunto $\left\{1, t, t^{2}, \ldots, t^{n}, \ldots\right\}$ é uma $\mathbb{Z}$-base para $\mathbb{Z} G$. Assim

$$
\mathbb{Z} G \cong \mathbb{Z}\left[x, x^{-1}\right]
$$

Não é difícil demonstrar que

$$
\begin{aligned}
P_{\bullet}^{\prime} \stackrel{\varepsilon}{\longrightarrow} \mathbb{Z}: \quad 0 \longrightarrow \mathbb{Z} G \stackrel{t-1}{\longrightarrow} \mathbb{Z} G \stackrel{\varepsilon}{\longrightarrow} \mathbb{Z} \longrightarrow 0, \\
g \stackrel{\longmapsto}{\longrightarrow}(t-1) \cdot g
\end{aligned}
$$

é uma resolução de $\mathbb{Z}$ sobre $G$. Aplicando o funtor $-\otimes_{G} \mathbb{Z}$, obtemos a sequência

$$
P_{\bullet}^{\prime} \otimes_{G} \mathbb{Z}: \quad 0 \longrightarrow \mathbb{Z} \stackrel{0}{\longrightarrow} \mathbb{Z} \longrightarrow 0
$$

portanto

$$
H_{n}(G) \cong \begin{cases}\mathbb{Z} & \text { se } n=0,1 \\ 0 & \text { se } n \neq 0,1\end{cases}
$$

Exemplo 1.13. Seja $X$ um conjunto e seja $G$ um grupo livre com base $X$. Note que todo elemento $g \in G$ pode ser escrito como uma palavra reduzida nos símbolos $\left\{x, x^{-1}: x \in X\right\}$. Definimos assim os subconjuntos $G(x)$ e $G\left(x^{-1}\right)$ como sendo todos os elementos $g \in G$ tais que a palavra reduzida que representa $g$ termina em $x$ ou $x^{-1}$, respetivamente. Note que $G-\{1\}$ é a união disjunta dos conjuntos $G(x)$ e $G\left(x^{-1}\right)$ sobre todos os $x \in X$. Logo

$$
\begin{aligned}
\text { se } g x \in G(x), \text { então } & (g x-1)=g(x-1)+(g-1), \\
\text { se } g x^{-1} \in G\left(x^{-1}\right), \text { então } & \left(g x^{-1}-1\right)=-\left(g x^{-1}\right)(x-1)+(g-1) .
\end{aligned}
$$

Fazendo indução no comprimento das palavras, reescrevemos a $\mathbb{Z}$-base $\{g-1: g \neq 1\} \subseteq I_{G}$ em termos do conjunto $\{g(x-1): g \in G, x \in X\}$. Logo $\{g(x-1): g \in G, x \in X\}$ é uma $\mathbb{Z}$-base de $I_{G}$ e portanto $\{x-1: x \in X\}$ é uma $\mathbb{Z} G$-base de $I_{G}$. Isto implica que $I_{G}$ é um $\mathbb{Z} G$-módulo livre e temos $I_{G} \cong \bigoplus_{x \in X} \mathbb{Z} G(x-1)$. Logo

$$
0 \longrightarrow I_{G} \stackrel{i}{\longrightarrow} \mathbb{Z} G \stackrel{\varepsilon}{\longrightarrow} \mathbb{Z} \longrightarrow 0
$$


é uma resolução livre de $\mathbb{Z}$ sobre $G$, onde $i: I_{G} \rightarrow \mathbb{Z} G$ é a inclusão. Aplicando o funtor $-\otimes_{G} \mathbb{Z}$ temos a sequência

$$
\begin{aligned}
0 \longrightarrow I_{G} \otimes_{G} \mathbb{Z} \longrightarrow & \mathbb{Z} G \otimes_{G} \mathbb{Z} \longrightarrow 0 \\
g(x-1) \otimes n \longmapsto & g(x-1) \otimes n=0 .
\end{aligned}
$$

Logo

$$
H_{0}(G)=\mathbb{Z}, \quad H_{1}(G)=I_{G} \otimes_{G} \mathbb{Z} \cong \bigoplus_{x \in X} \mathbb{Z}, \quad H_{n}(G)=0 \quad \text { sempre que } n>1 .
$$

EXEMPLO 1.14. Seja $M$ um $G$-módulo trivial e seja $P_{\bullet} \rightarrow \mathbb{Z}$ uma resolução livre de $\mathbb{Z}$ sobre $G$. Então

$$
H_{n}(G, M)=H_{n}\left(P \bullet \otimes_{G} M\right)=H_{n}\left(\left(P \bullet \otimes_{G} \mathbb{Z}\right) \otimes_{\mathbb{Z}} M\right)
$$

Como $P_{\bullet} \otimes_{G} \mathbb{Z}$ é um complexo de cadeias de $\mathbb{Z}$-módulos livres, usando o Teorema (1.6) temos

$$
\begin{aligned}
H_{n}(G, M) & \cong H_{n}\left(\left(P \bullet \otimes_{G} \mathbb{Z}\right) \otimes_{\mathbb{Z}} M\right) \cong\left(H_{n}\left(P \bullet \otimes_{G} \mathbb{Z}\right) \otimes_{\mathbb{Z}} M\right) \oplus \operatorname{Tor}_{1}^{\mathbb{Z}}\left(H_{n-1}\left(P \bullet \otimes_{G} \mathbb{Z}\right), M\right) \\
& \cong H_{n}(G) \otimes_{\mathbb{Z}} M \oplus \operatorname{Tor}_{1}^{\mathbb{Z}}\left(H_{n-1}(G), M\right) .
\end{aligned}
$$

Em particular se $n=1$, então

$$
\begin{aligned}
H_{1}(G, M) & \cong H_{1}(G) \otimes_{\mathbb{Z}} M \oplus \operatorname{Tor}_{1}^{\mathbb{Z}}\left(H_{0}(G), M\right)=\left(G /[G, G] \otimes_{\mathbb{Z}} M\right) \oplus \operatorname{Tor}_{1}^{\mathbb{Z}}(\mathbb{Z}, M) \\
& =G /[G, G] \otimes_{\mathbb{Z}} M .
\end{aligned}
$$

Exemplo 1.15. O exemplo que segue irá ser usado no Capítulo 4 para calcular os termos de uma sequência espectral importante.

Seja $\Sigma_{2}=\langle\sigma\rangle$ o grupo simétrico de ordem 2 e seja $A$ um grupo abeliano multiplicativo. O grupo $\Sigma_{2}$ age sobre $A \oplus A$ e sobre $A \otimes_{\mathbb{Z}} A$ da seguinte maneira

$$
\begin{aligned}
\Sigma_{2} \times(A \oplus A) & \longrightarrow A \oplus A \\
(\sigma,(a, b)) & \longmapsto \sigma(a, b)=(b, a), \\
\Sigma_{2} \times\left(A \otimes_{\mathbb{Z}} A\right) & \longrightarrow A \otimes_{\mathbb{Z}} A \\
(\sigma,(a \otimes b)) & \longmapsto \sigma(a \otimes b)=-b \otimes a .
\end{aligned}
$$

Aqui nós mostraremos

1) $H_{n}\left(\Sigma_{2}, A \oplus A\right) \cong \begin{cases}A & \text { se } n=0 \\ 0 & \text { se } n \neq 0\end{cases}$

2) $H_{n}\left(\Sigma_{2}, A \otimes_{\mathbb{Z}} A\right) \cong \begin{cases}\left(A \otimes_{\mathbb{Z}} A\right)_{\Sigma_{2}} & \text { se } n=0, \\ H_{n}\left(\Sigma_{2}, 2^{\infty} A \otimes_{\mathbb{Z}} 2^{\infty} A\right) & \text { se } n \text { é ímpar, } \\ \left\langle\overline{a \otimes a} \in\left(A \otimes_{\mathbb{Z}} A\right)_{\Sigma_{2}}: a \in A\right\rangle & \text { se } n \text { é par. }\end{cases}$ 
onde $2^{\infty} A:=\left\{a \in A\right.$ : existe $n \in \mathbb{N}$, tal que $\left.2^{n} a=0\right\}$.

De fato, como

$$
\sigma(a, b)-(a, b)=\left(a^{-1} b,\left(a^{-1} b\right)^{-1}\right)
$$

temos $(A \oplus A)_{\Sigma_{2}} \cong A$ e $(A \oplus A)^{\Sigma_{2}}=\{(a, a): a \in A\}$. Considere o homomorfismo

$$
N: A \oplus A \longrightarrow A \oplus A, \quad(a, b) \longmapsto(a, b)+\sigma(a, b)=(a b, a b) .
$$

Note que $\operatorname{Im} N=\{(a, a): a \in A\}=(A \oplus A)^{\Sigma_{2}}, \operatorname{Ker} N=\left\{\left(a, a^{-1}\right): a \in A\right\}$. Então temos um homomorfismo bem definido

$$
\bar{N}:(A \oplus A)_{\Sigma_{2}} \longrightarrow(A \oplus A)^{\Sigma_{2}}, \quad \overline{(a, b)} \longmapsto(a b, a b) .
$$

Dado que $(a, 1)-\sigma(a, 1)=\left(a, a^{-1}\right)$, então

$$
\operatorname{Ker} \bar{N}=0, \quad \operatorname{Im} \bar{N}=(A \oplus A)^{\Sigma_{2}} .
$$

Portanto pelo Exemplo (1.11)

$$
H_{n}\left(\Sigma_{2}, A \oplus A\right)= \begin{cases}A & \text { se } n=0 \\ 0 & \text { se } n \neq 0 .\end{cases}
$$

Para calcular $H_{n}\left(\Sigma_{2}, A \otimes_{\mathbb{Z}} A\right)$ note que pelo Exemplo (1.11): $H_{p}\left(\Sigma_{2}, A \otimes_{\mathbb{Z}} A\right) \cong \frac{\left(A \otimes_{\mathbb{Z}} A\right)^{\Sigma_{2}}}{(1+\sigma)\left(A \otimes_{\mathbb{Z}} A\right)}$ se $p$ é ímpar, e $H_{p}\left(\Sigma_{2}, A \otimes_{\mathbb{Z}} A\right) \cong \operatorname{Ker} \overline{N^{\prime}}$ se $p$ é par e $p \geq 2$, onde $\overline{N^{\prime}}:\left(A \otimes_{\mathbb{Z}} A\right)_{\Sigma_{2}} \rightarrow\left(A \otimes_{\mathbb{Z}} A\right)^{\Sigma_{2}}$, $\overline{a \otimes b} \mapsto a \otimes b-b \otimes a$.

Suponha agora que $A=A_{1} \oplus A_{2}$. Então

$$
A \otimes_{\mathbb{Z}} A \cong\left(A_{1} \otimes_{\mathbb{Z}} A_{1}\right) \oplus\left(A_{1} \otimes_{\mathbb{Z}} A_{2} \oplus A_{2} \otimes_{\mathbb{Z}} A_{1}\right) \oplus\left(A_{2} \otimes_{\mathbb{Z}} A_{2}\right) .
$$

A ação de $\Sigma_{2}$ sobre $A \otimes_{\mathbb{Z}} A$ induz uma ação de $\Sigma_{2}$ sobre $A_{1} \otimes_{\mathbb{Z}} A_{2} \oplus A_{2} \otimes_{\mathbb{Z}} A_{1}$ como segue

$$
\sigma\left(a_{1} \otimes a_{2}, b_{2} \otimes b_{1}\right)=-\left(b_{1} \otimes b_{2}, a_{2} \otimes a_{1}\right) .
$$

Pode-se mostrar que para $n>1, H_{n}\left(\Sigma_{2}, A_{1} \otimes_{\mathbb{Z}} A_{2} \oplus A_{2} \otimes_{\mathbb{Z}} A_{1}\right)=0$, portanto pelo Exemplo (1.7)

$$
H_{n}\left(\Sigma_{2}, A \otimes_{\mathbb{Z}} A\right)=H_{n}\left(\Sigma_{2}, A_{1} \otimes_{\mathbb{Z}} A_{2}\right) \oplus H_{n}\left(A_{2} \otimes_{\mathbb{Z}} A_{2}\right) .
$$

Agora se $A \cong \bigoplus_{i=1}^{n} A_{i}$, então por indução temos

$$
H_{k}\left(\Sigma_{2}, A \otimes_{\mathbb{Z}} A\right)=\bigoplus_{i=1}^{n} H_{k}\left(\Sigma_{2}, A_{i} \otimes_{\mathbb{Z}} A_{i}\right) .
$$

Mais geralmente se $\left\{A_{j}\right\}_{j \in J}$ é uma família de subgrupos finitamente gerados de $A$ tais que $J$ é um sistema direto e $A \cong \lim _{\longrightarrow} A_{j}$, então $A \otimes_{\mathbb{Z}} A \cong \underset{\longrightarrow}{\lim }\left(A_{j} \otimes_{\mathbb{Z}} A_{j}\right)$, e pelo Exemplo (1.5)

$H_{k}\left(\Sigma_{2}, A \otimes_{\mathbb{Z}} A\right)=\operatorname{Tor}_{k}^{\mathbb{Z} \Sigma_{2}}\left(\mathbb{Z}, \underline{\lim }\left(A_{j} \otimes_{\mathbb{Z}} A_{j}\right)\right) \cong \underline{\lim } \operatorname{Tor}_{k}^{\mathbb{Z} \Sigma_{2}}\left(\mathbb{Z}, A_{j} \otimes_{\mathbb{Z}} A_{j}\right)=\underline{\lim } H_{k}\left(\Sigma_{2}, A_{j} \otimes_{\mathbb{Z}} A_{j}\right)$.

Portanto é suficiente assumir que $A$ é finitamente gerado. Por outro lado, pelo teorema estrutural dos grupos abelianos finitamente gerados, basta assumir que $A$ é um grupo cíclico. 
- $\operatorname{Se} A \cong \mathbb{Z}$, então

$$
\begin{aligned}
\left(\mathbb{Z} \otimes_{\mathbb{Z}} \mathbb{Z}\right)^{\Sigma_{2}} & =\{n(1 \otimes 1): n(1 \otimes 1)=-n(1 \otimes 1), n \in \mathbb{Z}\} \\
& =\{n(1 \otimes 1): 2 n(1 \otimes 1)=0\} \\
& =0 . \\
\left(\mathbb{Z} \otimes_{\mathbb{Z}} \mathbb{Z}\right)_{\Sigma_{2}} & =\{\overline{n \otimes m}: n, m \in \mathbb{Z}\}=\{n m(\overline{1 \otimes 1}): n, m \in \mathbb{Z}\} \\
& =\langle\overline{1 \otimes 1}\rangle .
\end{aligned}
$$

Portanto

$$
H_{n}\left(\Sigma_{2}, \mathbb{Z} \otimes_{\mathbb{Z}} \mathbb{Z}\right)= \begin{cases}0 & \text { se } n \text { é impar, } \\ \langle\overline{1 \otimes 1}\rangle & \text { se } n \text { é par ou } n=0 .\end{cases}
$$

- Seja $A \cong \mathbb{Z} / n \mathbb{Z}$ e seja $n=2^{r_{0}} p_{1}^{r_{1}} \cdots p_{m}^{r_{m}} \operatorname{com} p_{i}$ primos, $p_{i}>2, p_{i} \neq p_{j}$ se $i \neq j$. Pela decomposição usual de $\mathbb{Z} / n \mathbb{Z}$ como sendo a soma direta $\mathbb{Z} / 2^{r_{0}} \mathbb{Z} \oplus \mathbb{Z} / p_{1}^{r_{1}} \mathbb{Z} \oplus \cdots \oplus \mathbb{Z} / p_{m}^{r_{m}} \mathbb{Z}$, é suficiente então assumir que $n=p^{r}$. Note que nosso caso, a $k$-ésima homologia $H_{k}(G,-)$ é anulado por $|G|=2$. Agora se $p$ é um primo ímpar, como $\mathbb{Z} / p^{r} \mathbb{Z} \otimes_{\mathbb{Z}} \mathbb{Z} / p^{r} \mathbb{Z}$ não tem 2-torção, então $H_{k}\left(\Sigma_{2}, \mathbb{Z} / p^{r} \mathbb{Z} \otimes_{\mathbb{Z}} \mathbb{Z} / p^{r} \mathbb{Z}\right)$ também não tem 2-torção. Assim

$$
H_{k}\left(\Sigma_{2}, \mathbb{Z} / p^{r} \otimes_{\mathbb{Z}} \mathbb{Z} / p^{r}\right)=0 .
$$

Por outro lado, se $n=2^{r}$, então a afirmação é clara. Portanto

$$
H_{n}\left(\Sigma_{2}, A \otimes_{\mathbb{Z}} A\right) \cong \begin{cases}\left(A \otimes_{\mathbb{Z}} A\right)_{\Sigma_{2}} & \text { se } n=0, \\ H_{n}\left(\Sigma_{2}, 2^{\infty} A \otimes_{\mathbb{Z}} 2^{\infty} A\right) & \text { se } n \text { é ímpar, } \\ \left\langle\overline{a \otimes a} \in\left(A \otimes_{\mathbb{Z}} A\right)_{\Sigma_{2}}: a \in A\right\rangle & \text { se } n \text { é par } .\end{cases}
$$

A seguir, apresentamos algumas construções importantes de resoluções livres de $\mathbb{Z}$ sobre qualquer grupo, utilizadas mais diante.

- Seja $G^{(n)}$ o produto cartesiano de $n$ cópias do grupo $G$. Seja $C_{n}(G)$ o $\mathbb{Z}$-módulo livre sobre $G^{(n+1)}$ junto com a ação de $G$ dada por

$$
g \cdot\left(g_{0}, g_{1}, \ldots, g_{n}\right):=\left(g g_{0}, g g_{1}, \ldots, g g_{n}\right) .
$$

$C_{n}(G)$ é um $G-$ módulo livre com base $\left(1, g_{1}, g_{1} g_{2}, \ldots, g_{1} g_{2} \ldots g_{n}\right), g_{i} \in G$. É claro que $C_{0}(G) \cong \mathbb{Z} G$, via o isomorfismo $n_{g_{0}}\left(g_{0}\right) \mapsto n_{g_{0}} g_{0}$. Considere para todo $n \geq 1$ os homomorfismos $\delta_{n}: C_{n}(G) \rightarrow C_{n-1}(G)$ definidos como sendo

$$
\delta_{n}\left(g_{0}, \ldots, g_{n}\right):=\sum_{i=0}^{n}(-1)^{i}\left(g_{0}, \ldots, g_{i-1}, g_{i+1}, \ldots, g_{n}\right) .
$$

O complexo

$C_{\bullet}(G) \stackrel{\varepsilon}{\longrightarrow} \mathbb{Z}: \quad \cdots \rightarrow C_{n}(G) \stackrel{\delta_{n}}{\longrightarrow} C_{n-1}(G) \longrightarrow \cdots \longrightarrow C_{1}(G) \stackrel{\delta_{1}}{\longrightarrow} C_{0}(G) \stackrel{\varepsilon}{\longrightarrow} \mathbb{Z} \rightarrow 0$,

é uma resolução livre de $G$-módulos e é chamada resolução padrão de $\mathbb{Z}$ sobre $G$. Note que $\varepsilon: C_{0}(G) \rightarrow \mathbb{Z}$ é o homomorfismo de augmentação, $\varepsilon\left(n_{g}(g)\right)=n_{g}$. 
- Agora vamos introduzir a notação barra:

$$
\left[g_{1}\left|g_{2}\right| \ldots \mid g_{n}\right], \quad g_{i} \in G-\{1\} .
$$

Além disso, suponha que $\left[g_{1}\left|g_{2}\right| \ldots \mid g_{n}\right]=0$, sempre que $g_{i}=1$ para algum $i$ entre 1 e $n$. Considere $B_{n}(G)$ como o $G$-módulo livre gerado por todos os símbolos $\left[g_{1}\left|g_{2}\right| \ldots \mid g_{n}\right]$ com $g_{i} \in G-\{1\}$. Para $n \geq 1$, definimos os homomorfismos $d_{n}: B_{n}(G) \rightarrow B_{n-1}(G)$, como $d_{n}\left(\left[g_{1}|\ldots| g_{n}\right]\right):=g_{1}\left[g_{2}|\ldots| g_{n}\right]+\sum_{i=1}^{n-1}(-1)^{i}\left[g_{1}|\ldots| g_{i} g_{i+1}|\ldots| g_{n}\right]+(-1)^{n}\left[g_{1}|, \ldots,| g_{n-1}\right]$.

Note que $B_{0}(G)$ é o $G$-módulo livre com gerador [ ], que é isomorfo a $\mathbb{Z} G$. Portanto o complexo de cadeias de $G$-módulos

$$
B_{\bullet}(G) \stackrel{\varepsilon}{\longrightarrow} \mathbb{Z}: \quad \cdots \longrightarrow B_{n}(G) \stackrel{d_{n}}{\longrightarrow} B_{n-1}(G) \rightarrow \cdots \rightarrow B_{1}(G) \stackrel{d_{1}}{\longrightarrow} B_{0}(G) \stackrel{\varepsilon}{\longrightarrow} \mathbb{Z} \rightarrow 0
$$

é uma resolução livre de $\mathbb{Z}$ sobre $G$ chamada resolução barra de $G[15$, Capítulo 6 , Seção 5], onde $\varepsilon: B_{0}(G) \rightarrow \mathbb{Z}$ é o homomorfismo de augmentação.

Podemos verificar que o homomorfismo

$$
\begin{aligned}
C_{n}(G) & \stackrel{\theta_{n}}{\longrightarrow} B_{n}(G) \\
\left(g_{0}, \ldots, g_{n}\right) & \longmapsto\left[g_{0}^{-1} g_{1}\left|g_{1}^{-1} g_{2}\right| \cdots \mid g_{n-1}^{-1} g_{n}\right]
\end{aligned}
$$

dá-nos um morfismo de complexoss $\theta_{\bullet}: C_{\bullet}(G) \rightarrow B_{\bullet}(G)$. Note que

$$
\theta_{n}\left(1, g_{1}, g_{1} g_{2}, \cdots, g_{1} \ldots g_{n}\right)=\left[g_{1}\left|g_{2}\right| \cdots \mid g_{n}\right]
$$

Assim, se $M$ é um $G$-módulo, então

$$
H_{n}(G, M) \cong H_{n}\left(C_{\bullet}(G) \otimes_{G} M\right) \cong H_{n}\left(B \bullet(G) \otimes_{G} M\right)
$$

EXEMPLO 1.16. Seja $G$ um grupo abeliano e considere $B_{\bullet}(G) \rightarrow \mathbb{Z}$ a resolução barra de $\mathbb{Z}$ sobre $G$. Considere a aplicação

$$
\begin{aligned}
G \times G & \longrightarrow B_{2}(G) \otimes_{G} \mathbb{Z} . \\
\left(g_{1}, g_{2}\right) & \longmapsto\left[g_{1} \mid g_{2}\right] \otimes 1-\left[g_{2} \mid g_{1}\right] \otimes 1
\end{aligned}
$$

Como $\left(d_{2} \otimes i d_{\mathbb{Z}}\right)\left(\left[g_{1} \mid g_{2}\right] \otimes 1-\left[g_{2} \mid g_{1}\right] \otimes 1\right)=0$, temos assim um homomorfismo bem definido

$$
\begin{aligned}
G \times G & \longrightarrow H_{2}(G) \\
\left(g_{1}, g_{2}\right) & \longmapsto \overline{\left[g_{1} \mid g_{2}\right] \otimes 1}-\overline{\left[g_{2} \mid g_{1}\right] \otimes 1}
\end{aligned}
$$

Esta aplicação é bilinear alternante, logo induz um único homomorfismo

$$
\begin{aligned}
\mathbf{c}: \wedge_{\mathbb{Z}}^{2} G & \longrightarrow H_{2}(G) \\
g_{1} \wedge g_{2} & \longmapsto \mathbf{c}\left(g_{1}, g_{2}\right):=\overline{\left[g_{1} \mid g_{2}\right] \otimes 1}-\overline{\left[g_{2} \mid g_{1}\right] \otimes 1} .
\end{aligned}
$$


Portanto, para todo $g_{1}, g_{2}, h_{1} \in G$ temos as seguintes propriedades

$$
\mathbf{c}\left(g_{1} h_{1}, g_{2}\right)=\mathbf{c}\left(g_{1}, g_{2}\right)+\mathbf{c}\left(h_{1}, g_{2}\right), \quad \mathbf{c}\left(g_{2}, g_{1}\right)=-\mathbf{c}\left(g_{1}, g_{2}\right) \text {. }
$$

Além disso, segundo [1, Teorema 6.4 Ch. V], o homomorfismo $\mathbf{c}$ induz o isomorfismo

$$
H_{2}(G) \cong \bigwedge_{\mathbb{Z}}^{2} G
$$

OBSERVAÇÃo 1.1. Em geral, para um grupo qualquer $G$ definimos

$$
\mathbf{c}\left(g_{1}, g_{2}, \ldots, g_{n}\right):=\sum_{\sigma \in \Sigma_{n}} \operatorname{sign}(\sigma) \overline{\left[g_{\sigma(1)}\left|g_{\sigma(2)}\right| \ldots \mid g_{\sigma(n)}\right] \otimes 1} \in H_{n}(G),
$$

onde $g_{i} \in G$ é tal que $g_{i} g_{j}=g_{j} g_{i}$ para todo $j \neq i$ e $\Sigma_{n}$ é o grupo simétrico de grau $n$. É fácil verificar que na verdade $\mathbf{c}\left(g_{1}, g_{2}, \ldots, g_{n}\right) \in H_{n}(G)$, como foi obtido para $n=2$ no Exemplo (1.16). Se $A=\left\langle g_{1}, g_{2} \ldots, g_{n}\right\rangle$, não é difícil verificar que o homomorfismo

$$
\begin{aligned}
\wedge^{n} A & \longrightarrow H_{n}(G), \\
a_{1} \wedge \cdots \wedge a_{n} & \longmapsto \mathbf{c}\left(a_{1}, \ldots, a_{n}\right)
\end{aligned}
$$

está bem definido e é multilinear. Portanto esses elementos satisfazem as seguintes propriedades

- Se $h_{1} \in G$ comuta com os elementos $g_{1}, g_{2}, \ldots, g_{n} \in G$, então

$$
\mathbf{c}\left(g_{1} h_{1}, g_{2}, \ldots, g_{n}\right)=\mathbf{c}\left(g_{1}, g_{2}, \ldots, g_{n}\right)+\mathbf{c}\left(h_{1}, g_{2}, \ldots, g_{n}\right) \text {. }
$$

- Para cada $\sigma \in \Sigma_{n}$

$$
\mathbf{c}\left(g_{\sigma(1)}, g_{\sigma(2)}, \ldots, g_{\sigma(n)}\right)=\operatorname{sign}(\sigma) \mathbf{c}\left(g_{1}, g_{2}, \ldots, g_{n}\right)
$$

- Sejam $G$ e $H$ grupos. Então o produto cup (Proposição 1.3) de c $\left(g_{1}, \ldots, g_{n}\right) \in H_{n}(G)$ e $\mathbf{c}\left(h_{1}, \ldots, h_{m}\right) \in H_{m}(H)$ é dado por

$$
\mathbf{c}\left(g_{1}, \ldots, g_{n}\right) \cup \mathbf{c}\left(h_{1}, \ldots, h_{m}\right)=\mathbf{c}\left(\left(g_{1}, 1\right), \ldots,\left(g_{n}, 1\right),\left(1, h_{1}\right), \ldots,\left(1, h_{m}\right)\right) .
$$

Usamos mais diante no Capítulo 4 esses elementos e suas propriedades.

Agora estudaremos os homomorfismos induzidos nos grupos de homologia pelos homomorfismos entre grupos. Sejam $G$ e $G^{\prime}$ grupos multiplicativos e sejam $M$ e $M^{\prime}$ quaisquer $G$-módulo e $G^{\prime}$-módulo respetivamente. Um morfismo $(\alpha, f):(G, M) \rightarrow\left(G^{\prime}, M^{\prime}\right)$ está formado por um homomorfismo de grupos $\alpha: G \rightarrow G^{\prime}$ e um homomorfismo de grupos abelianos $f: M \rightarrow M^{\prime}$ tal que para todo $g \in G$ e para todo $m \in M$,

$$
f(g \cdot m)=\alpha(g) \cdot f(m) .
$$

Considere agora $P_{\bullet} \stackrel{\varepsilon}{\longrightarrow} \mathbb{Z}$ e $P_{\bullet}^{\prime} \stackrel{\varepsilon^{\prime}}{\longrightarrow} \mathbb{Z}$ resoluções projetivas de $\mathbb{Z}$ sobre $G$ e $G^{\prime}$ respetivamente. Dado que existe uma extensão de $\alpha$ para um homomorfismo de anéis $\mathbb{Z} G \rightarrow \mathbb{Z} G^{\prime}$, então $P_{\bullet}^{\prime}$ é um 
complexo de cadeias de $G$-módulos e pelo Lema (1.1) existe um morfismo de complexos de cadeias, único a menos de homotopia, $\tau_{\bullet}: P_{\bullet} \rightarrow P_{\bullet}^{\prime}$ compatível com $\alpha$, isto é para todo $g \in G$ e para todo $x \in P_{n}$

$$
\tau_{n}(g \cdot x)=\alpha(g) \cdot \tau_{n}(x)
$$

que satisfaz $\varepsilon^{\prime} \circ \tau_{0}=\varepsilon$. Tensorizando com $M$, obtemos um morfismo de complexos de cadeias

$$
\tau_{\bullet} \otimes_{G} f: P_{\bullet} \otimes_{G} M \rightarrow P_{\bullet}^{\prime} \otimes_{G^{\prime}} M^{\prime}
$$

Desta forma temos induzido para cada $n \geq 0$ um homomorfismo entre os $n$-ésimos grupos de homologia

$$
(\alpha, f)_{*}: H_{n}(G, M) \longrightarrow H_{n}\left(G^{\prime}, M^{\prime}\right), \quad \overline{z_{n}} \longmapsto \overline{\left(\tau_{n} \otimes f\right)\left(z_{n}\right)} .
$$

Se $H \subseteq G$ é um subgrupo de $G$ e $M$ é um $G$-módulo, o homomorfismo natural $H_{n}(H, M) \rightarrow$ $H_{n}(G, M)$ induzido pela inclusão $(H, M) \rightarrow(G, M)$, é chamado o homomorfismo correstrição, denotado por

$$
\operatorname{cor}_{G}^{H}: H_{n}(H, M) \rightarrow H_{n}(G, M) .
$$

Seja $H \subseteq G$ um subgrupo de $G$ e seja $E$ um conjunto de representantes das classes à direita $H g$. Para qualquer $g \in E$, seja $\mathbb{Z}(H g)$ o $\mathbb{Z}$-módulo livre com base indexada por elementos na classe à direita $H g$. Então $\mathbb{Z}(H g)$ é um $H$-módulo usando $h^{\prime} \cdot \sum a_{h} h_{g}=\sum a_{h} h^{\prime} h_{g}$ e portanto $\mathbb{Z} H \cong \mathbb{Z}(H g)$ (como $H$-módulos) via o homomorfismo $h \mapsto h g$. Segue-se

$$
\mathbb{Z} G=\bigoplus_{g \in E} \mathbb{Z}(H g) \cong \bigoplus_{g \in E} \mathbb{Z} H
$$

assim, $\mathbb{Z} G$ é um $\mathbb{Z} H$-módulo livre com base indexada em $E$. Agora, se $P_{\bullet} \rightarrow \mathbb{Z}$ é uma resolução livre de $\mathbb{Z}$ sobre $G$, dado que $\mathbb{Z} G$ é um $\mathbb{Z} H$-módulo livre, então $P_{\bullet} \rightarrow \mathbb{Z}$ é uma resolução livre de $\mathbb{Z}$ sobre $H$. Logo para todo $n \geq 0$ e todo $G-$ módulo $M$

$$
H_{n}(G, M)=H_{n}\left(P_{\bullet} \otimes_{G} M\right), \quad H_{n}(H, M)=H_{n}\left(P_{\bullet} \otimes_{H} M\right) .
$$

EXEMPLO 1.17. Seja $H \subseteq G$ subgrupo de $G$ e seja $\theta: G / H \rightarrow G$ qualquer seção (como aplicação de conjuntos) da aplicação $\pi: G \rightarrow G / H$ definida por $g \mapsto g H$. Sejam $C_{\bullet}(H) \rightarrow \mathbb{Z}, C_{\bullet}(G) \rightarrow \mathbb{Z}$ resoluções padrão de $\mathbb{Z}$ sobre $H$ e $G$ respetivamente. Para todo $g \in G$, definimos

$$
\bar{g}:=(\theta(\pi(g)))^{-1} g \in H .
$$

A aplicação

$$
\begin{aligned}
C_{n}(G) & \longrightarrow C_{n}(H) \\
\left(g_{0}, g_{1}, \ldots, g_{n}\right) & \longmapsto\left(\overline{g_{0}}, \overline{g_{1}}, \ldots, \overline{g_{n}}\right)
\end{aligned}
$$

induz um morfismo de complexos de cadeias $\left(C_{\bullet}(G), \delta_{\bullet}\right) \rightarrow\left(C_{\bullet}(H), \delta_{\bullet}^{\prime}\right)$ tal que para todo $n \in \mathbb{Z}$ o homomorfismo $H_{n}(H)=H_{n}\left(C_{\bullet}(G) \otimes_{H} \mathbb{Z}\right) \rightarrow H_{n}\left(C_{\bullet}(H) \otimes_{H} \mathbb{Z}\right)=H_{n}(H)$ coincide com a identidade $i d_{H_{n}(H)}$, sendo esta a inversa do homomorfismo induzido pela inclusão $i_{\bullet}: C_{\bullet}(H) \rightarrow$ $C \bullet(G)$

$$
H_{n}(H)=H_{n}\left(C_{\bullet}(H) \otimes_{H} \mathbb{Z}\right) \stackrel{i_{*}=i d_{H_{n}(H)}}{\longrightarrow} H_{n}\left(C_{\bullet}(G) \otimes_{H} \mathbb{Z}\right)=H_{n}(H)
$$


EXEMPLO 1.18. Seja $H \subseteq G$ subgrupo de $G$ e $M$ um $G$-módulo. Fixe $g_{0} \in G$ e considere o homomorfismo de grupos

$$
\alpha_{g_{0}}: H \longrightarrow g_{0} H g_{0}^{-1}, \quad h \longmapsto g_{0} h g_{0}^{-1},
$$

e o homomorfismo de grupos abelianos

$$
f_{g_{0}}: M \longrightarrow M, \quad m \longmapsto g_{0} \cdot m
$$

Seja $P_{\bullet} \rightarrow \mathbb{Z}$ uma resolução de $\mathbb{Z}$ sobre $G$ e seja $\left(\tau_{g_{0}}\right)_{n}: P_{n} \rightarrow P_{n}$ dada por $\left(\tau_{g_{0}}\right)_{n}(x)=g_{0} \cdot x$. Como para todo $h \in H$ e para todo $x \in M$

$$
\left(\tau_{g_{0}}\right)_{n}(h \cdot x)=g_{0} \cdot(h \cdot x)=\left(g_{0} h g_{0}^{-1}\right) \cdot\left(g_{0} \cdot x\right)=\alpha_{g_{0}}(h) \cdot \tau_{n}(x),
$$

temos o morfismo

$$
\begin{aligned}
\left(\tau_{g_{0}}\right) \bullet \otimes_{H} f_{g_{0}}: P \bullet \otimes_{H} M & \longrightarrow P_{\bullet} \otimes_{g_{0} H g_{0}^{-1}} M . \\
x \otimes m & \longmapsto g_{0} \cdot x \otimes g_{0} \cdot m=g_{0} \cdot(x \otimes m)
\end{aligned}
$$

Logo, para todo $n \geq 0$,

$$
\left(\alpha_{g_{0}}, f_{g_{0}}\right)_{*}: H_{n}(H, M) \longrightarrow H_{n}\left(g_{0} H g_{0}^{-1}, M\right), \quad \overline{z_{n}} \longmapsto \overline{g_{0} \cdot\left(z_{n}\right)} .
$$

Em particular, se $g_{0} \in H$ então para todo $\overline{z_{n}} \in H_{n}(H, M)$, temos $\left(\alpha_{g_{0}}, f_{g_{0}}\right)_{*}\left(\overline{z_{n}}\right)=\overline{z_{n}}$, portanto

$$
\left(\alpha_{g_{0}}, f_{g_{0}}\right)_{*}=i d_{H_{n}(H, M)}
$$

Assim, se $H$ é um subgrupo normal de $G$, então a ação por conjugação de $G$ sobre $(H, M)$ definida por

$$
G \times(H, M) \longrightarrow(H, M), \quad\left(g_{0},(h, m)\right) \longmapsto\left(g_{0} h g_{0}^{-1}, g_{0} \cdot m\right)
$$

induz, para todo $n \geq 0$, uma ação de $G / H$ sobre $H_{n}(H, M)$ dada por

$$
G / H \times H_{n}(H, M) \longrightarrow H_{n}(H, M), \quad\left(\overline{g_{0}}, x\right) \longmapsto\left(\alpha_{g_{0}}, f_{g_{0}}\right)(x)
$$

Logo, para cada $n \geq 0, H_{n}(H, M)$ é um $G / H$-módulo.

EXEMPLO 1.19. Seja $R$ um anel com unidade. Definimos o grupo linear geral de ordem $n$, denotado $G L_{n}(R)$, como o grupo de matrizes invertíveis de ordem $n \times n$ com coeficientes no anel $R$. Em particular, $R^{\times} \cong G L_{1}(R)$. Se $R$ é comutativo, definimos o subgrupo especial linear de ordem $n$, como sendo $S L_{n}(R):=\operatorname{Ker} \operatorname{det}_{n}$ onde $\operatorname{det}_{n}: G L_{n}(R) \longrightarrow R^{\times}$é o homomorfismo determinante. Note que para cada $n \geq 1$, o homomorfismo determinante tem uma seção dada por

$$
\begin{aligned}
\phi_{n}: R^{\times} & \longrightarrow G L_{n}(R) . \\
x & \longmapsto\left(\begin{array}{cc}
x & 0 \\
0 & I_{n-1}
\end{array}\right)
\end{aligned}
$$


Por outro lado, identificando cada matriz invertível $A$ de ordem $n \times n$ com a matriz $\left(\begin{array}{ll}A & 0 \\ 0 & 1\end{array}\right)$ de ordem $(n+1) \times(n+1)$, obtemos uma inclusão $G L_{n}(R) \rightarrow G L_{n+1}(R)$. A união da cadeia

$$
R^{\times}=G L_{1}(R) \subset G L_{2}(R) \subset \cdots \subset G L_{n}(R) \subset G L_{n+1}(R) \subset \cdots
$$

é chamada o grupo linear geral de $R$

$$
G L(R):=\bigcup_{n \geq 1} G L_{n}(R)
$$

Igualmente, definimos $S L(R)$ como a união dos grupos $S L_{n}(R)$ e estendemos o homomorfismo determinante det $_{n}$ para o homomorfismo det $: G L(R) \rightarrow R^{\times}$. Assim $S L(R)=\operatorname{Ker}$ det e $G L(R)$ é o produto semidireto $S L(R) \rtimes R^{\times}$. Pelo Exemplo (1.18), para cada $n \geq 1$ temos uma ação de $R^{\times}$ sobre $H_{n}(S L(R))$ induzida pela conjugação

$$
S L(R) \longrightarrow S L(R), \quad A \longmapsto x \cdot A \cdot x^{-1}=\left(\begin{array}{ccc}
x & 0 & \ldots \\
0 & 1 & \ldots \\
\vdots & \vdots & \ddots
\end{array}\right) A\left(\begin{array}{ccc}
x^{-1} & 0 & \ldots \\
0 & 1 & \ldots \\
\vdots & \vdots & \ddots
\end{array}\right)
$$

onde $x \in R^{\times}$. Se $A \in S L(R)$, então existe $n \in \mathbb{Z}$ tal que $A \in S L_{n}(R)$. Logo para cada $x \in R^{\times}$ definimos

$$
x_{n}:=\left(\begin{array}{cccc}
x & 0 & \cdots & 0 \\
0 & 1 & \cdots & 0 \\
\vdots & \vdots & \ddots & \vdots \\
0 & 0 & \cdots & 1
\end{array}\right) \in G L_{n}(R)
$$

Então

$$
x_{2 n}=\left(\begin{array}{c|c}
x_{n} & 0 \\
\hline 0 & x_{n}^{-1}
\end{array}\right)\left(\begin{array}{c|c}
I_{n} & 0 \\
\hline 0 & x_{n}
\end{array}\right), \quad x_{2 n}^{-1}=\left(\begin{array}{c|c}
I_{n} & 0 \\
\hline 0 & x_{n}^{-1}
\end{array}\right)\left(\begin{array}{c|c}
x_{n}^{-1} & 0 \\
\hline 0 & x_{n}
\end{array}\right)
$$

Assim

$$
\begin{aligned}
x \cdot A \cdot x^{-1} & =\left(\begin{array}{ccc}
x & 0 & \cdots \\
0 & 1 & \cdots \\
\vdots & \vdots & \ddots
\end{array}\right) A\left(\begin{array}{ccc}
x^{-1} & 0 & \cdots \\
0 & 1 & \cdots \\
\vdots & \vdots & \ddots
\end{array}\right) \\
& =x_{2 n}\left(\begin{array}{c|c}
A & 0 \\
\hline 0 & I_{n}
\end{array}\right) x_{2 n}^{-1}=\left(\begin{array}{c|c}
x_{n} & 0 \\
\hline 0 & x_{n}^{-1}
\end{array}\right)\left(\begin{array}{c|c}
A & 0 \\
\hline 0 & I_{n}
\end{array}\right)\left(\begin{array}{c|c}
x_{n} & 0 \\
\hline 0 & x_{n}^{-1}
\end{array}\right)^{-1} .
\end{aligned}
$$

Isto implica que a ação induzida por $x \in R^{\times}$é a mesma que a ação induzida por $\left(\begin{array}{c|c}x_{n} & 0 \\ \hline 0 & x_{n}^{-1}\end{array}\right) \in$ $S L(R)$. Portanto para cada $n \geq 1, R^{\times}$age trivialmente sobre $H_{n}(S L(R))$.

LEMA 1.4 (Lema de Shapiro). Seja $H \subseteq G$ subgrupo de G e seja M um H-módulo. Então

$$
H_{n}(H, M) \cong H_{n}\left(G, \mathbb{Z} G \otimes_{H} M\right)
$$

onde o isomorfismo é induzido por $($ inc,$\alpha):(H, M) \rightarrow\left(G, \mathbb{Z} G \otimes_{H} M\right), \alpha(m)=1 \otimes m$. 
Demonstração. Seja $P_{\bullet} \rightarrow \mathbb{Z}$ uma resolução projetiva de $\mathbb{Z}$ sobre $G$. Usando o isomorfismo $P \bullet \otimes_{G}\left(\mathbb{Z} G \otimes_{H} M\right) \cong P_{\bullet} \otimes_{H} M$, temos

$$
H_{n}\left(G, \mathbb{Z} G \otimes_{H} M\right) \cong H_{n}\left(P_{\bullet} \otimes_{G}\left(\mathbb{Z} G \otimes_{H} M\right)\right) \cong H_{n}\left(P_{\bullet} \otimes_{H} M\right) \cong H_{n}(H, M) .
$$

Se $G$ e $H$ são dois grupos, então temos os homomorfismos de anéis

$$
\begin{array}{ll}
\mathbb{Z} G \longrightarrow \mathbb{Z}[G \times H], & \sum_{g \in G} n_{g} g \longmapsto \sum_{g \in G} n_{g}(g, 1), \\
\mathbb{Z} H \longrightarrow \mathbb{Z}[G \times H], & \sum_{h \in H} n_{h} h \longmapsto \sum_{h \in H} n_{h}(1, h),
\end{array}
$$

que induzem o homomorfismo de anéis

$$
\mathbb{Z} G \otimes_{\mathbb{Z}} \mathbb{Z} H \longrightarrow \mathbb{Z}[G \times H]
$$

Como os dois anéis tem a $G \times H$ como $\mathbb{Z}$-base, então

$$
\mathbb{Z}[G \times H] \cong \mathbb{Z} G \otimes_{\mathbb{Z}} \mathbb{Z} H
$$

Assim, se $P_{\bullet}$ e $Q \bullet$ são complexos de cadeias de $\mathbb{Z} G$-módulos e $\mathbb{Z} H$-módulos, respetivamente, então

$$
\begin{aligned}
P_{\bullet} \otimes_{\mathbb{Z}} Q \bullet & \cong\left(P_{\bullet} \otimes_{G} \mathbb{Z} G\right) \otimes_{\mathbb{Z}}\left(\mathbb{Z} H \otimes_{H} Q \bullet\right) \cong P_{\bullet} \otimes_{G}\left(\mathbb{Z} G \otimes_{\mathbb{Z}} \mathbb{Z} H\right) \otimes_{H} Q_{\bullet} \\
& \cong P_{\bullet} \otimes_{G}\left(Q_{\bullet} \otimes_{H}\left(\mathbb{Z} G \otimes_{\mathbb{Z}} \mathbb{Z} H\right)\right) \cong\left(P_{\bullet} \otimes_{G} \mathbb{Z}\right) \otimes_{\mathbb{Z}}\left(Q_{\bullet} \otimes_{H} \mathbb{Z}[G \times H]\right) .
\end{aligned}
$$

Logo

$$
\left(P \bullet \otimes_{\mathbb{Z}} Q_{\bullet}\right) \otimes_{G \times H} \mathbb{Z} \cong\left[\left(P_{\bullet} \otimes_{G} \mathbb{Z}\right) \otimes_{\mathbb{Z}}\left(Q \bullet \otimes_{H} \mathbb{Z}[G \times H]\right)\right] \otimes_{G \times H} \mathbb{Z} \cong\left(P_{\bullet} \otimes_{G} \mathbb{Z}\right) \otimes_{\mathbb{Z}}\left(Q_{\bullet} \otimes_{H} \mathbb{Z}\right) .
$$

Em particular, se $P_{\bullet}$ é uma resolução livre de $\mathbb{Z}$ sobre $G$ e $Q_{\bullet}$ é uma resolução livre de $\mathbb{Z}$ sobre $H$, então pelo Corolário (1.1) o complexo $P_{\bullet} \otimes_{\mathbb{Z}} Q \bullet$ é uma resolução livre de $\mathbb{Z}$ sobre $G \times H$ e temos

$$
H_{n}(G \times H) \cong H_{n}\left(\left(P_{\bullet} \otimes_{\mathbb{Z}} Q_{\bullet}\right) \otimes_{G \times H} \mathbb{Z}\right)=H_{n}\left(\left(P_{\bullet} \otimes_{G} \mathbb{Z}\right) \otimes_{\mathbb{Z}}\left(Q_{\bullet} \otimes_{H} \mathbb{Z}\right)\right) .
$$

Dado que cada $P_{n} \otimes_{G} \mathbb{Z}$ é um $\mathbb{Z}$-módulo livre, então pelo Corolário (1.1) temos o seguinte resultado.

Proposição 1.3 (Fórmula de Künneth para Homología de grupos). Para todos os grupos $G e$ $H$, existe uma sequência exata

$$
0 \longrightarrow \bigoplus_{p+q=n} H_{p}(G) \otimes H_{q}(H) \stackrel{\cup}{\longrightarrow} H_{n}(G \times H) \longrightarrow \bigoplus_{p^{\prime}+q^{\prime}=n-1} \operatorname{Tor}_{1}^{\mathbb{Z}}\left(H_{p^{\prime}}(G), H_{q^{\prime}}(H)\right) \longrightarrow 0
$$

que cinde. O homomorfismo $\cup: H_{p}(G) \otimes H_{q}(H) \rightarrow H_{p+q}(G \times H)$ é chamado o produto cup.

EXEMPLO 1.20. Sejam $G$ e $H$ grupos (multiplicativos). 
- Se $n=1$, então

$$
\begin{aligned}
H_{1}(G \times H) & \cong\left(H_{0}(G) \otimes_{\mathbb{Z}} H_{1}(H) \oplus H_{1}(G) \otimes_{\mathbb{Z}} H_{0}(H)\right) \oplus \operatorname{Tor}_{1}^{\mathbb{Z}}\left(H_{0}(G), H_{0}(H)\right) \\
& \cong H_{1}(G) \oplus H_{1}(H) .
\end{aligned}
$$

- Se $n=2$, então $\operatorname{Tor}_{1}^{\mathbb{Z}}\left(\mathbb{Z}, H_{1}(H)\right)=\operatorname{Tor}_{1}^{\mathbb{Z}}\left(H_{1}(G), \mathbb{Z}\right)=0$, logo

$$
H_{2}(G \times H) \cong H_{2}(G) \oplus\left(H_{1}(G) \otimes_{\mathbb{Z}} H_{1}(H)\right) \oplus H_{2}(H) .
$$

Em particular, se $A$ é um grupo abeliano

$$
H_{2}(A \times A) \cong H_{2}(A) \oplus H_{2}(A) \oplus\left(A \otimes_{\mathbb{Z}} A\right)
$$

Seja $F$ um corpo e considere $\mu(F):=\lim _{\rightarrow} \mu_{n}(F)$, onde $\mu_{n}(F)=\left\{\xi \in F^{\times}: \xi^{n}=1\right\} \cong \mathbb{Z} / n \mathbb{Z}$ é um grupo cíclico finito. O seguinte lema dá-nos uma descrição da terceira homologia do grupo $F^{\times} \times F^{\times}$usando a terceira homologia do grupo $\mu(F) \times \mu(F)$, isto está muito relacionado com os cálculos que faremos no Capítulo 4 para demonstrar nosso teorema principal.

LEMA 1.5. Seja F um corpo. Então pela fórmula de Künneth temos uma decomposição canônica

$$
H_{3}\left(F^{\times} \times F^{\times}\right) \cong\left(\bigoplus_{i+j=3} H_{i}\left(F^{\times}\right) \otimes_{\mathbb{Z}} H_{j}\left(F^{\times}\right)\right) \oplus \operatorname{Tor}_{1}^{\mathbb{Z}}\left(F^{\times}, F^{\times}\right),
$$

onde o homomorfismo que cinde $\operatorname{Tor}_{1}^{\mathbb{Z}}\left(F^{\times}, F^{\times}\right) \cong \operatorname{Tor}_{1}^{\mathbb{Z}}(\mu(F), \mu(F)) \rightarrow H_{3}\left(F^{\times} \times F^{\times}\right)$está definido por $\langle\xi, n, \xi\rangle \mapsto \chi(\xi)$, onde $\mu_{n}(F)=\langle\xi\rangle, \xi^{n}=1,\langle\xi, n, \xi\rangle \in \operatorname{Tor}_{1}^{\mathbb{Z}}\left(\mu_{n}(F), \mu_{n}(F)\right)$ é a imagem de $\xi$ via o isomorfismo $\mu_{n}(F) \cong \operatorname{Tor}_{1}^{\mathbb{Z}}\left(\mu_{n}(F), \mu_{n}(F)\right)$ e

$$
\begin{aligned}
\chi(\xi):= & \sum_{i=1}^{n}\left(\left[(\xi, 1)|(1, \xi)|\left(1, \xi^{i}\right)\right]-\left[(1, \xi)|(\xi, 1)|\left(1, \xi^{i}\right)\right]+\left[(1, \xi)\left|\left(1, \xi^{i}\right)\right|(\xi, 1)\right]\right. \\
& \left.+\left[(\xi, 1)\left|\left(\xi^{i}, 1\right)\right|(1, \xi)\right]-\left[(\xi, 1)|(1, \xi)|\left(\xi^{i}, 1\right)\right]+\left[(1, \xi)|(\xi, 1)|\left(\xi^{i}, 1\right)\right]\right) .
\end{aligned}
$$

Demonstração. Como $\mu(F)=\lim _{\rightarrow} \mu_{n}(F)$, pela Proposição (1.2) e pelo Exemplo (1.11)

$$
H_{2}(\mu(F))=H_{2}\left(\lim _{\rightarrow} \mu_{n}(F)\right)=\lim _{\rightarrow} H_{2}\left(\mu_{n}(F)\right)=0 .
$$

Então pela Fórmula de Künneth (1.3) sobre $H_{3}(\mu(F) \times \mu(F))$ temos a seguinte sequência exata que cinde

$$
0 \longrightarrow H_{3}(\mu(F)) \oplus H_{3}(\mu(F)) \stackrel{\alpha}{\longrightarrow} H_{3}(\mu(F) \times \mu(F)) \longrightarrow \operatorname{Tor}_{1}^{\mathbb{Z}}(\mu(F), \mu(F)) \longrightarrow 0 .
$$

Aqui $\alpha: H_{3}(\mu(F)) \oplus H_{3}(\mu(F)) \rightarrow H_{3}(\mu(F) \times \mu(F))$ está definido como $\alpha=H_{3}\left(i_{1}\right)+H_{3}\left(i_{2}\right)$, onde $i_{j}: \mu(F) \rightarrow \mu(F) \times \mu(F)$ é a inclusão usual de $\mu(F)$ na $j$-ésima posição de $\mu(F) \times \mu(F)$. Agora considere as projeções

$$
\begin{aligned}
\beta_{1}: \mu(F) \times \mu(F) & \longrightarrow \mu(F), & \beta_{2}: \mu(F) \times \mu(F) & \longrightarrow \mu(F), \\
(\xi, \tau) & \longmapsto \xi & (\xi, \tau) & \longmapsto \tau
\end{aligned}
$$


Se $\beta=H_{3}\left(\beta_{1}\right) \oplus H_{3}\left(\beta_{2}\right)$, onde

$$
H_{3}\left(\beta_{1}\right): H_{3}(\mu(F) \times \mu(F)) \longrightarrow H_{3}(\mu(F)), \quad H_{3}\left(\beta_{2}\right): H_{3}(\mu(F) \times \mu(F)) \longrightarrow H_{3}(\mu(F)),
$$

então $\beta \circ \alpha=i d_{H_{3}(\mu(F)) \oplus H_{3}(\mu(F))}$. Assim, a sequência exata (1.9) cinde e temos a decomposição canônica

$$
H_{3}(\mu(F) \times \mu(F)) \cong H_{3}(\mu(F)) \oplus H_{3}(\mu(F)) \oplus \operatorname{Tor}_{1}^{\mathbb{Z}}(\mu(F), \mu(F)) .
$$

Por outro lado, seja $B_{\bullet}(\mu(F))$ a resolução barra de $\mathbb{Z}$ sobre $\mu(F)$. Como $\mu(F) \cong t\left(F^{\times}\right)$e

$$
\operatorname{Tor}_{1}^{\mathbb{Z}}(\mu(F), \mu(F)) \cong \lim _{\rightarrow} \operatorname{Tor}_{1}^{\mathbb{Z}}\left(\mu_{n}(F), \mu_{n}(F)\right)
$$

os elementos de $\operatorname{Tor}_{1}^{\mathbb{Z}}(\mu(F), \mu(F)) \cong \operatorname{Tor}_{1}^{\mathbb{Z}}\left(H_{1}\left(F^{\times}\right), H_{1}\left(F^{\times}\right)\right) \cong \operatorname{Tor}_{1}^{\mathbb{Z}}\left(F^{\times}, F^{\times}\right)$são da forma $\langle\xi, n, \xi\rangle:=\langle[\xi], n,[\xi]\rangle$, onde $\xi \in \mu_{n}(F)$, isto é $\xi^{n}=1$ e $[\xi] \in H_{1}\left(F^{\times}\right) \cong F^{\times}$. Note que o homomorfismo

$$
\begin{aligned}
\partial_{2}: B_{2}(\mu(F))_{\mu(F)} & \longrightarrow B_{1}(\mu(F))_{\mu(F)}, \\
\sum_{i=1}^{n}\left[\xi, \xi^{i}\right] & \longmapsto \sum_{i=1}^{n}\left[\xi^{i}\right]-\sum_{i=1}^{n}\left[\xi^{i}\right]+\sum_{i=1}^{n}[\xi]=n[\xi]
\end{aligned}
$$

assim, segundo [4, Proposição 10.6, Capítulo 5] obtemos um homomorfismo

$$
\begin{aligned}
\phi: \operatorname{Tor}_{1}^{\mathbb{Z}}\left(H_{1}(\mu(F)), H_{1}(\mu(F))\right) & \longrightarrow H_{3}\left(B_{\bullet}(\mu(F))_{\mu(F)} \otimes B_{\bullet}(\mu(F))_{\mu(F)}\right) \\
\langle[\xi], n,[\xi]\rangle & \longmapsto[\xi] \otimes \sum_{i=1}^{n}\left[\xi \mid \xi^{i}\right]+\sum_{i=1}^{n}\left[\xi \mid \xi^{i}\right] \otimes[\xi]
\end{aligned}
$$

que cinde a sequência (1.9). Agora pelo isomorfismo (1.7)

$$
B_{\bullet}(\mu(F))_{\mu(F)} \otimes_{\mathbb{Z}} B_{\bullet}(\mu(F))_{\mu(F)} \stackrel{\psi}{\cong}\left(B_{\bullet}(\mu(F)) \otimes_{\mathbb{Z}} B_{\bullet}(\mu(F))\right)_{\mu(F) \times \mu(F)}
$$

temos

$$
\begin{aligned}
\psi\left([\xi] \otimes \sum_{i=1}^{n}\left[\xi \mid \xi^{i}\right]\right) & =\sum_{i=1}^{n}[(\xi, 1)]\left[(1, \xi) \mid\left(1, \xi^{i}\right)\right] \\
& =\sum_{i=1}^{n}\left[(\xi, 1)|(1, \xi)|\left(1, \xi^{i}\right)\right]-\left[(1, \xi)|(\xi, 1)|\left(1, \xi^{i}\right)\right]+\left[(1, \xi)\left|\left(1, \xi^{i}\right)\right|(\xi, 1)\right], \\
\psi\left(\sum_{i=1}^{n}\left[\xi \mid \xi^{i}\right] \otimes[\xi]\right) & =\sum_{i=1}^{n}\left[(\xi, 1) \mid\left(\xi^{i}, 1\right)\right][(1, \xi)] \\
& =\sum_{i=1}^{n}\left[(\xi, 1)\left|\left(\xi^{i}, 1\right)\right|(1, \xi)\right]-\left[(\xi, 1)|(1, \xi)|\left(\xi^{i}, 1\right)\right]+\left[(1, \xi)|(\xi, 1)|\left(\xi^{i}, 1\right)\right] .
\end{aligned}
$$

Assim

$$
\begin{aligned}
\phi: \operatorname{Tor}_{1}^{\mathbb{Z}}\left(H_{1}(\mu(F)), H_{1}(\mu(F))\right) & \longrightarrow H_{3}(\mu(F) \times \mu(F)), \\
\langle[\xi], n,[\xi]\rangle & \longmapsto \chi(\xi),
\end{aligned}
$$

cinde a sequência (1.9), onde

$$
\begin{aligned}
\chi(\xi):= & \sum_{i=1}^{n}\left(\left[(\xi, 1)|(1, \xi)|\left(1, \xi^{i}\right)\right]-\left[(1, \xi)|(\xi, 1)|\left(1, \xi^{i}\right)\right]+\left[(1, \xi)\left|\left(1, \xi^{i}\right)\right|(\xi, 1)\right]\right. \\
& \left.+\left[(\xi, 1)\left|\left(\xi^{i}, 1\right)\right|(1, \xi)\right]-\left[(\xi, 1)|(1, \xi)|\left(\xi^{i}, 1\right)\right]+\left[(1, \xi)|(\xi, 1)|\left(\xi^{i}, 1\right)\right]\right) .
\end{aligned}
$$


Por outro lado, usando a fórmula de Künneth (1.3) sobre $H_{3}\left(F^{\times} \times F^{\times}\right)$temos a seguinte sequência exata

$$
0 \longrightarrow M \stackrel{\cup}{\longrightarrow} H_{3}\left(F^{\times} \times F^{\times}\right) \longrightarrow \operatorname{Tor}_{1}^{\mathbb{Z}}\left(F^{\times}, F^{\times}\right) \longrightarrow 0,
$$

onde $M:=H_{3}\left(F^{\times}\right) \oplus H_{3}\left(F^{\times}\right) \oplus\left(F^{\times} \otimes H_{2}\left(F^{\times}\right)\right) \oplus\left(H_{2}\left(F^{\times}\right) \otimes F^{\times}\right) \subseteq H_{3}\left(F^{\times} \times F^{\times}\right)$. Esta sequência cinde usando o homomorfismo $\phi: \operatorname{Tor}_{1}^{\mathbb{Z}}(\mu(F), \mu(F)) \rightarrow H_{3}(\mu(F) \times \mu(F))$ definido anteriormente para a decomposição canônica de $H_{3}(\mu(F) \times \mu(F))$ e usando o seguinte diagrama comutativo

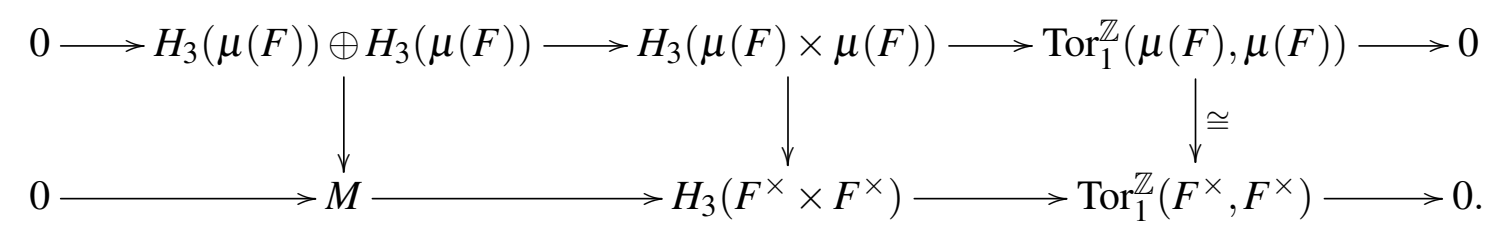

\section{$1.3 \mathrm{O}$ grupo $\operatorname{Ext}_{\mathbb{Z}}^{1}(B, A)$}

Sejam $A$ e $B$ grupos abelianos. Uma extensão de $B$ por $A$ é uma sequência exata de grupos abelianos $0 \longrightarrow A \stackrel{\chi}{\longrightarrow} C \stackrel{\sigma}{\longrightarrow} B \longrightarrow 0$. Denotaremos esta sequência como $E=(\chi, \sigma)$. Um morfismo entre $E=(\chi, \sigma)$ e $E^{\prime}=\left(\chi, \sigma^{\prime}\right)$ extensões de $B$ por $A$ é a terna $\Gamma=(\alpha, \beta, \gamma): E \rightarrow E^{\prime}$ de homomorfismos tal que o seguinte diagrama comuta

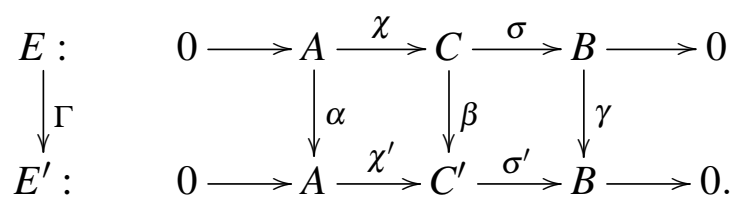

Dizemos que $E=(\chi, \sigma)$ e $E^{\prime}=\left(\chi^{\prime}, \sigma^{\prime}\right)$ são congruentes e denotamos $E \equiv E^{\prime}$, se existe um morfismo $\Gamma=\left(i d_{A}, \beta, i d_{B}\right): E \rightarrow E^{\prime}$ tal que $\beta$ é um isomorfismo. Esta relação definida entre extensões de $B$ por $A$ é uma relação de equivalencia e denotamos

$$
[E]:=\left\{E^{\prime}: E^{\prime}=\left(\chi^{\prime}, \sigma^{\prime}\right) \text { é uma extensão de } B \text { por } A \text { tal que } E \equiv E^{\prime}\right\} .
$$

Dada uma extensão $E=(\chi, \sigma)$ de $B$ por $A$ e homomorfismos $f: A \rightarrow A^{\prime}, g: B^{\prime} \rightarrow B$, então existem extensões $E^{\prime}$ de $B$ por $A^{\prime}$ e $E^{\prime \prime}$ de $B^{\prime}$ por $A$ (definidas únicamente por $f$ e $g$ ), e morfismos de extensões $\Gamma^{\prime}: E \rightarrow E^{\prime}$, e $\Gamma^{\prime \prime}: E^{\prime \prime} \rightarrow E$ tais que os seguintes diagramas comutam

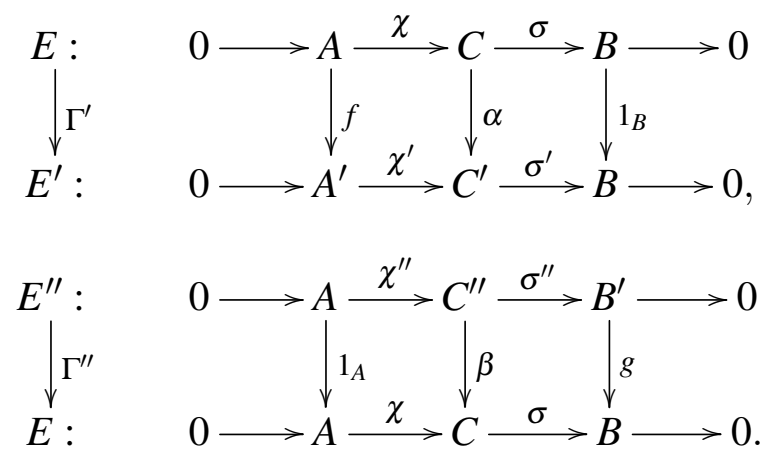


Denotaremos as extensões $E^{\prime}$ e $E^{\prime \prime}$ como sendo as composições da extensão $E$ com $f$ e $g$, isto é $E^{\prime}=f \circ E$ e $E^{\prime \prime}=E \circ g$. Agora dados $A, B$ grupos abelianos, defina os homomorfismos

$$
\begin{gathered}
\nabla_{A}: A \oplus A \rightarrow A, \quad \nabla_{A}\left(a_{1}, a_{2}\right)=a_{1}+a_{2}, \\
\Delta_{B}: B \rightarrow B \oplus B, \quad \Delta_{B}(b)=(b, b) .
\end{gathered}
$$

Dadas as seguintes extensões de $B$ por $A$

$$
\begin{array}{cl}
E: & 0 \longrightarrow A \stackrel{\chi}{\longrightarrow} C_{1} \stackrel{\sigma}{\longrightarrow} B \longrightarrow 0, \\
E^{\prime}: & 0 \longrightarrow A \stackrel{\chi^{\prime}}{\longrightarrow} C_{2} \stackrel{\sigma^{\prime}}{\longrightarrow} B \longrightarrow 0
\end{array}
$$

definimos a soma direta de $E$ e $E^{\prime}$ como sendo a extensão

$$
E \oplus E^{\prime}: \quad 0 \longrightarrow A \oplus A \stackrel{\chi \oplus \chi^{\prime}}{\longrightarrow} C_{1} \oplus C_{2} \stackrel{\sigma \oplus \sigma^{\prime}}{\longrightarrow} B \oplus B \longrightarrow 0,
$$

e definimos a soma nas classes de extensões $[E]$ e $\left[E^{\prime}\right]$ como sendo $[E]+\left[E^{\prime}\right]:=\left[E+E^{\prime}\right]$ onde $E+E^{\prime}:=\nabla_{A} \circ\left(E \oplus E^{\prime}\right) \circ \Delta_{B}$.

TEOREMA 1.9. Sejam A e B grupos abelianos. Então o conjunto

$$
\operatorname{Ext}_{\mathbb{Z}}^{1}(B, A):=\{[E]: E=(\chi, \sigma) \text { é uma extensão de B por } A\},
$$

é um grupo abeliano junto com a soma sobre as classes de extensões e é chamado o o grupo de extensões de B por A.

Demonstração. Veja-se [4, Teorema 2.1, Capítulo 3].

ObSERVAÇão 1.2. Seja $E=(i, p)$ a extensão de $B$ por $A$, onde $i$ é a inclusão de $A$ em $A \oplus B$ e $p$ é a projeção de $A \oplus B$ sobre $B$. Esta extensão é chamada extensão trivial, e a classe dele é o elemento trivial de $\operatorname{Ext}_{\mathbb{Z}}^{1}(B, A):[0]=[E] \in \operatorname{Ext}_{\mathbb{Z}}^{1}(B, A)$. Uma extensão $E^{\prime}=\left(\chi^{\prime}, \sigma^{\prime}\right)$ é dita que cinde se $E^{\prime}$ é congruente com a extensão trivial, equivalentemente $E^{\prime}$ é dita que cinde se $\sigma^{\prime}$ tem inversa a direita ou se $\chi^{\prime}$ tem inversa a esquerda. Por outro lado, dada uma extensão $[E] \in \operatorname{Ext}_{\mathbb{Z}}^{1}(B, A)$ definimos uma nova extensão de $B$ por $A$ como sendo $E^{\prime}:=\left(-1_{A}\right) \circ E$, onde $-1_{A}(a)=-a$. Esta extensão $E^{\prime}$ satisfaz: $[E]+\left[E^{\prime}\right]=[0]=\left[E^{\prime}\right]+[E]$, logo $E^{\prime}$ é a extensão inversa de $E$ em $\operatorname{Ext}_{\mathbb{Z}}^{1}(B, A)$, denotado como $\left[E^{\prime}\right]=-[E]$. Em diante denotaremos as classes de extensões de $E$ simplesmente por $E$.

Seja $A=2 \mathbb{Z}$ e $B=\mathbb{Z} / 2 \mathbb{Z}$. É fácil verificar que $\operatorname{Ext}_{\mathbb{Z}}^{1}(\mathbb{Z} / 2 \mathbb{Z}, 2 \mathbb{Z}) \cong \mathbb{Z} / 2 \mathbb{Z}$. Em geral temos o seguinte teorema.

TEOREMA 1.10. Para qualquer grupo abeliano A, temos que

$$
\operatorname{Ext}_{\mathbb{Z}}^{1}(\mathbb{Z} / n \mathbb{Z}, A) \cong A / n A,
$$

onde $n A=\{n a: a \in A\}$. 
Demonstração. Veja-se [4, Proposição 1.1, Capítulo 3].

EXEMPLO 1.21. Em particular tem-se que

$$
\operatorname{Ext}_{\mathbb{Z}}^{1}(\mathbb{Z} / 2 \mathbb{Z}, \mathbb{Z} / n \mathbb{Z}) \cong\left\{\begin{array} { l l } 
{ 0 } & { \text { se } 2 \nmid n } \\
{ \mathbb { Z } / 2 \mathbb { Z } } & { \text { se } 2 | n }
\end{array} \text { e } \quad \operatorname { E x t } _ { \mathbb { Z } } ^ { 1 } ( \mathbb { Z } / n \mathbb { Z } , \mathbb { Z } / 2 \mathbb { Z } ) \cong \left\{\begin{array}{ll}
0 & \text { se } 2 \nmid n \\
\mathbb{Z} / 2 \mathbb{Z} & \text { se } 2 \mid n
\end{array}\right.\right.
$$

Se $2 \mid n$, considere as seguintes sequências exatas

$$
E: \quad 0 \longrightarrow \mathbb{Z} / n \mathbb{Z} \stackrel{\alpha}{\longrightarrow} \mathbb{Z} /(2 n) \mathbb{Z} \stackrel{\beta}{\longrightarrow} \mathbb{Z} / 2 \mathbb{Z} \longrightarrow 0,
$$

onde $\alpha(\bar{j})=\overline{2 j}$ e $\beta(\bar{m})=\bar{m}$ e

$$
E^{\prime}: 0 \longrightarrow \mathbb{Z} / 2 \mathbb{Z} \stackrel{\alpha^{\prime}}{\longrightarrow} \mathbb{Z} /(2 n) \mathbb{Z} \stackrel{\beta^{\prime}}{\longrightarrow} \mathbb{Z} / n \mathbb{Z} \longrightarrow 0,
$$

onde $\alpha^{\prime}(\overline{1})=\bar{n}, \beta^{\prime}(\bar{a})=\bar{a}$. Então $E \in \operatorname{Ext}_{\mathbb{Z}}^{1}(\mathbb{Z} / 2 \mathbb{Z}, \mathbb{Z} / n \mathbb{Z})$ e $E^{\prime} \in \operatorname{Ext}_{\mathbb{Z}}^{1}(\mathbb{Z} / n \mathbb{Z}, \mathbb{Z} / 2 \mathbb{Z})$. Dado que $2 \mid n$, então $\mathbb{Z} /(2 n) \mathbb{Z} \nsucceq \mathbb{Z} / 2 \mathbb{Z} \oplus \mathbb{Z} / n \mathbb{Z}$. Portanto $E$ e $E^{\prime}$ são as únicas extensões não triviais de $\operatorname{Ext}_{\mathbb{Z}}^{1}(\mathbb{Z} / 2 \mathbb{Z}, \mathbb{Z} / n \mathbb{Z})$ e de $\operatorname{Ext}_{\mathbb{Z}}^{1}(\mathbb{Z} / n \mathbb{Z}, \mathbb{Z} / 2 \mathbb{Z})$ respetivamente.

EXEMPLO 1.22. Seja $F$ um corpo. Sabemos que

$$
\operatorname{Tor}_{1}^{\mathbb{Z}}(\mu(F), \mu(F)) \cong \lim _{\rightarrow} \operatorname{Tor}_{1}^{\mathbb{Z}}\left(\mu_{n}(F), \mu_{n}(F)\right) \cong \lim _{\rightarrow} \mu_{n}(F)=\mu(F)
$$

Então $\operatorname{Ext}_{\mathbb{Z}}^{1}\left(\mathbb{Z} / 2 \mathbb{Z}, \operatorname{Tor}_{1}^{\mathbb{Z}}(\mu(F), \mu(F))\right) \cong \operatorname{Ext}_{\mathbb{Z}}^{1}(\mathbb{Z} / 2 \mathbb{Z}, \mu(F))$. Dado que todo grupo abeliano de torção $A$ pode ser decomposto na forma $\bigoplus_{p \text { primo }} p^{\infty} A$, onde $p^{\infty} A=\left\{a \in A\right.$ : existe $r$ tal que $\left.a^{p^{r}}=1\right\}$, então pelo Teorema (1.10) temos

$$
\operatorname{Ext}_{\mathbb{Z}}^{1}(\mathbb{Z} / 2 \mathbb{Z}, \mu(F)) \cong \operatorname{Ext}_{\mathbb{Z}}^{1}\left(\mathbb{Z} / 2 \mathbb{Z}, \mu_{2^{\infty}}(F)\right) \cong \frac{\mu_{2^{\infty}}(F)}{\mu_{2^{\infty}}^{2}(F)} .
$$

Agora se char $(F)=2$, então $\mu_{2^{\infty}}(F)$ é trivial. Por outro lado, se char $(F) \neq 2$ e se $\mu_{2^{\infty}}(F)$ é infinito, então para qualquer $n$ temos que $\mu_{2^{n}}(F) \subseteq \mu_{2^{n+1}}(F)$, onde $\mu_{2^{n}}(F)=\langle\xi\rangle$ com $\xi \in F^{\times}$ uma $2^{n}$-ésima raiz primitiva da unidade e $\mu_{2^{n+1}}(F)=\langle\eta\rangle$ com $\eta \in F^{\times}$uma $2^{n+1}$-ésima raiz primitiva da unidade. É claro que $\xi=\eta^{2}, \log \frac{\mu_{2^{\infty}}(F)}{\mu_{2^{\infty}}^{2}(F)}=0$. Se char $(F) \neq 2$ e $\mu_{2^{\infty}}(F)$ é finito, então $\mu_{2^{\infty}}(F)=\mu_{2^{n}}(F)$ para algum inteiro $n$. Neste caso $\frac{\mu_{2^{\infty}}(F)}{\mu_{2^{\infty}}^{2}(F)} \cong \mathbb{Z} / 2 \mathbb{Z}$ e portanto

$$
\operatorname{Ext}_{\mathbb{Z}}^{1}\left(\mathbb{Z} / 2 \mathbb{Z}, \operatorname{Tor}_{1}^{\mathbb{Z}}(\mu(F), \mu(F))\right) \cong \begin{cases}0 & \text { se } \operatorname{char}(F)=2 \text { ou se } \mu_{2^{\infty}}(F) \text { for infinito } \\ \mathbb{Z} / 2 \mathbb{Z} & \text { se } \operatorname{char}(F) \neq 2 \text { e } \mu_{2^{\infty}}(F) \text { for finito }\end{cases}
$$

Definimos assim $\operatorname{Tor}_{1}^{\mathbb{Z}}(\mu(F), \mu(F))^{\sim}$ como sendo a única extensão não trivial de $\mathbb{Z} / 2 \mathbb{Z}$ por $\operatorname{Tor}_{1}^{\mathbb{Z}}(\mu(F), \mu(F))$ se char $(F) \neq 2$ e $\mu_{2^{\infty}}(F)$ for finito. Caso contrário definimos $\operatorname{Tor}_{1}^{\mathbb{Z}}(\mu(F), \mu(F))^{\sim}$ como sendo $\operatorname{Tor}_{1}^{\mathbb{Z}}(\mu(F), \mu(F))$. Voltaremos nesse exemplo no final do Capítulo 4. 


\subsection{Sequências Espectrais}

Uma sequência espectral começando em $a \in \mathbb{Z}, a \geq 0$ é uma família $\left\{E_{p, q}^{r}\right\}_{p, q \in \mathbb{Z}}$ de $R$-módulos com $r \geq a$, junto com $R$-homomorfismos

$$
d_{p, q}^{r}: E_{p, q}^{r} \longrightarrow E_{p-r, q+r-1}^{r}
$$

tais que $d_{p, q}^{r} \circ d_{p+r, q-r+1}^{r}=0$ e $E_{p, q}^{r+1} \cong \frac{\operatorname{Ker} d_{p, q}^{r}}{\operatorname{Im} d_{p+r, q-r+1}^{r}}$

Para cada $r \geq a$, a família $\left\{E_{p, q}^{r}\right\}_{p, q \in \mathbb{Z}}$ é chamada a $r$-ésima página ou folha da sequência espectral. Os homomorfismos $d_{p, q}^{r}$ são chamados as diferenciais da sequência espectral.

EXEMPLO 1.23. Seja $\left\{E_{p, q}^{r}\right\}_{p, q \in \mathbb{Z}}$ uma sequência espectral começando em 0. A folha zero $(r=0)$ desta sequência espectral é

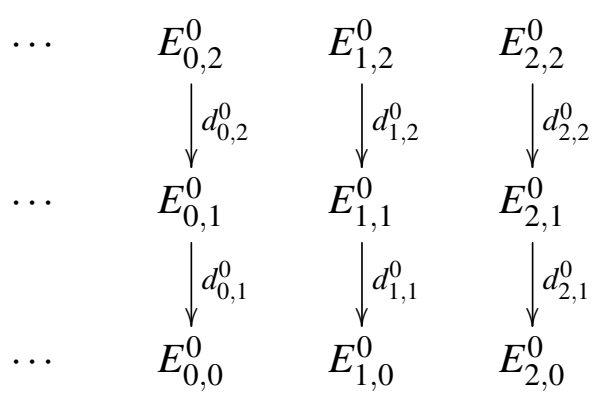

A primeira folha $(r=1)$ desta sequência espectral é

$$
\begin{aligned}
& \cdots \quad \stackrel{d_{0,2}^{1}}{\longleftarrow} E_{0,2}^{1} \stackrel{d_{1,2}^{1}}{\longleftarrow} E_{1,2}^{1} \stackrel{d_{2,2}^{1}}{\longleftarrow} E_{2,2}^{1} \longleftarrow \quad \cdots \\
& \cdots \quad \stackrel{d_{0,1}^{1}}{\longleftarrow} E_{0,1}^{1} \stackrel{d_{1,1}^{1}}{\longleftarrow} E_{1,1}^{1} \stackrel{d_{2,1}^{1}}{\longleftarrow} E_{2,1}^{1} \longleftarrow \quad \cdots \\
& \cdots \quad \stackrel{d_{0,0}^{1}}{\longleftarrow} E_{0,0}^{1} \stackrel{d_{1,0}^{1}}{\longleftarrow} E_{1,0}^{1} \stackrel{d_{2,0}^{1}}{\longleftarrow} E_{2,0}^{1} \longleftarrow \quad \cdots
\end{aligned}
$$

e a segunda folha $(r=2)$ desta sequência espectral é

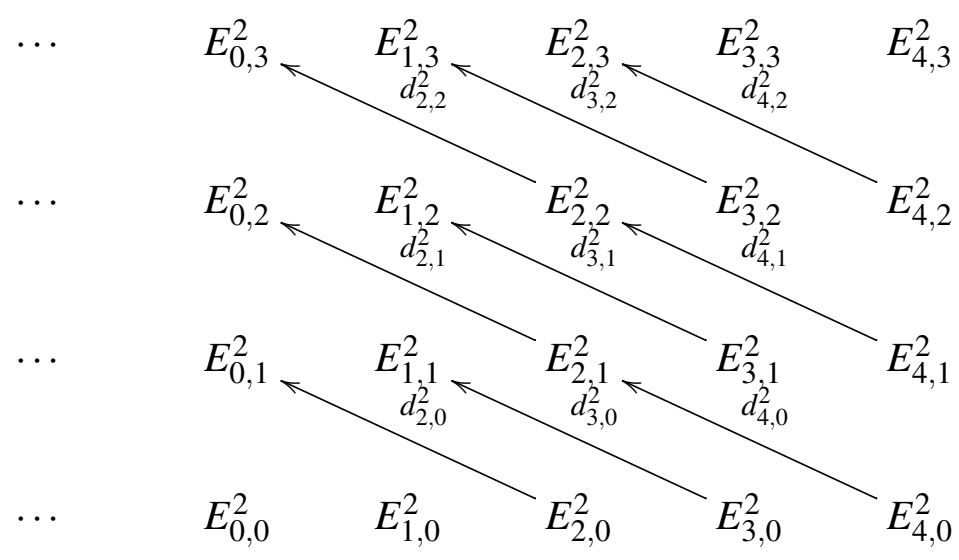


Uma sequência espectral é dita limitada se para cada $n \in \mathbb{Z}$ existem somente um número finito de termos $E_{p, q}^{r}$ diferentes de zero com $p+q=n$. Se assim for, então para cada $p$ e $q$ existe $r_{0} \in \mathbb{N}$ tal que $E_{p, q}^{r} \cong E_{p, q}^{r+1}$ para todo $r \geq r_{0}$. Denotamos o termo estável por $E_{p, q}^{\infty}$. A partir de agora para referir a uma sequência espectral usamos somente a notação $E_{p, q}^{r}$.

Seja $H_{\bullet}=\left\{H_{n}\right\}_{n \in \mathbb{Z}}$ uma família de $R$-módulos. Dizemos que uma sequência espectral limitada $E_{p, q}^{r}$ (começando em $a \geq 0$ ) converge a $H_{\bullet}$ se para cada $n \in \mathbb{Z}$, existem $s, t \in \mathbb{Z}$ tais que $H_{n}$ tem uma filtração da forma

$$
0=F_{s} H_{n} \subseteq \cdots \subseteq F_{p-1} H_{n} \subseteq F_{p} H_{n} \subseteq F_{p+1} H_{n} \subseteq \cdots \subseteq F_{t} H_{n}=H_{n}
$$

tal que para todo $p, q$,

$$
E_{p, q}^{\infty} \cong \frac{F_{p} H_{p+q}}{F_{p-1} H_{p+q}}
$$

No caso afirmativo, denotaremos esta sequência espectral como

$$
E_{p, q}^{a} \Longrightarrow H_{p+q}
$$

EXEMPLO 1.24. Seja $E_{p, q}^{r} \Longrightarrow H_{p+q}$ uma sequência espectral tal que $E_{p, q}^{2}=0$ para todo $p \neq 0,1$. É fácil verificar que $E_{p, q}^{\infty} \cong E_{p, q}^{2}$ para todo $p$ e $q$. Para cada $n$, a sequência espectral $E_{p, q}^{r}$ induz uma filtração de $H_{n}$ da forma

$$
0=F_{S} H_{n} \subseteq \cdots \subseteq F_{p-1} H_{n} \subseteq F_{p} H_{n} \subseteq F_{p+1} H_{n} \subseteq \cdots \subseteq F_{t} H_{n}=H_{n}
$$

de modo que

$$
E_{p, n-p}^{\infty} \cong \frac{F_{p} H_{n}}{F_{p-1} H_{n}}
$$

Se $p<0$, então $E_{p, n-p}^{\infty}=0$ e logo

$$
0=F_{s} H_{n}=\cdots=F_{p-1} H_{n}=F_{p} H_{n}=\cdots=F_{-1} H_{n} .
$$

Se $p=0$, então

$$
E_{0,2}^{2} \cong E_{0, n}^{\infty} \cong \frac{F_{0} H_{n}}{F_{-1} H_{n}}=F_{0} H_{n}
$$

Se $p>1$, então

$$
F_{1} H_{n}=\cdots=F_{p-1} H_{n}=F_{p} H_{n}=\cdots=F_{t} H_{n}=H_{n} .
$$

Se $p=1$, então

$$
E_{1, n-1}^{2} \cong E_{1, n-1}^{\infty} \cong \frac{F_{1} H_{n}}{F_{0} H_{n}}=\frac{H_{n}}{E_{0, n}^{2}} .
$$

Assim, para cada $n \in \mathbb{Z}$ obtemos a seguinte sequência exata curta

$$
0 \longrightarrow E_{0, n}^{2} \longrightarrow H_{n} \longrightarrow E_{1, n-1}^{2} \longrightarrow 0
$$


EXEMPLO 1.25. Seja $E_{p, q}^{r} \Longrightarrow H_{p+q}$ tal que $E_{p, q}^{2}=0$ para todo $q \neq 0,1$. É fácil verificar que

$$
E_{p, q}^{\infty} \cong\left\{\begin{array}{lc}
\operatorname{Ker} d_{p, 0}^{2} & \text { se } q=0 \\
\frac{E_{p, 1}^{2}}{\operatorname{Im} d_{p+2,0}^{2}} & \text { se } q=1 \\
0 & \text { se } q \neq 0,1 .
\end{array}\right.
$$

Para todo $n \in \mathbb{Z}, H_{n}$ tem uma filtração da forma

$$
0=F_{s} H_{n} \subseteq \cdots \subseteq F_{p-1} H_{n} \subseteq F_{p} H_{n} \subseteq F_{p+1} H_{n} \subseteq \cdots \subseteq F_{t} H_{n}=H_{n}
$$

tal que

$$
E_{n, n-p}^{\infty} \cong \frac{F_{p} H_{n}}{F_{p-1} H_{n}}
$$

Se $n<p$, então $E_{n, n-p}^{\infty}=0$ e logo

$$
F_{n} H_{n}=F_{n+1} H_{n}=\cdots=F_{p-1} H_{n}=F_{p} H_{n}=\cdots=F_{t} H_{n}=H_{n} .
$$

Se $n=p$, então

Se $n>p+1$, então

$$
\frac{F_{n} H_{n}}{F_{n-1} H_{n}}=\frac{H_{n}}{F_{n-1} H_{n}} \cong E_{n, 0}^{\infty} \cong \operatorname{Ker} d_{n, 0}^{2}
$$

$$
0=F_{s} H_{n}=\cdots=F_{p-1} H_{n}=F_{p} H_{n}=\cdots=F_{n-2} H_{n} .
$$

Se $n=p+1$, então

$$
\frac{F_{n-1} H_{n}}{F_{n-2} H_{n}}=F_{n-1} H_{n} \cong E_{n-1,1}^{\infty} \cong \frac{E_{n-1,1}^{2}}{\operatorname{Im} d_{n+1,0}^{2}} .
$$

Obtemos assim para cada $n \in \mathbb{Z}$ a sequência exata

$$
0 \rightarrow E_{n-1,1}^{3} \rightarrow H_{n} \rightarrow E_{n, 0}^{2} \rightarrow E_{n-2,1}^{2} \rightarrow E_{n-2,1}^{3} \rightarrow 0 .
$$

Fazendo variar $n$ e juntando as sequências obtemos a seguinte sequência exata longa

$$
\cdots \rightarrow H_{n-1} \rightarrow E_{n+1,0}^{2} \rightarrow E_{n-1,1}^{2} \rightarrow H_{n} \rightarrow E_{n, 0}^{2} \rightarrow E_{n-2,1}^{2} \rightarrow H_{n-1} \rightarrow \cdots .
$$

Uma sequência espectral tal que $E_{p, q}^{r}=0$ para todo $p<0$ ou $q<0$ é chamada a sequência espectral do primeiro quadrante. Logo para cada $n \geq 0$, os termos $E_{p, q}^{r}$ diferentes de zero tais que $p+q=n$ são ao máximo $n+1$. Portanto esta sequência espectral é limitada. Então para $p, q \in \mathbb{Z}$ fixos, se $r_{0}=\max \{p, q+1\}+1$, então $E_{p, q}^{\infty}=E_{p, q}^{r_{0}}$.

EXEMPLO 1.26. Seja $E_{p, q}^{r} \Longrightarrow H_{p+q}$ uma sequência espectral do primeiro quadrante começando em $a$. Então para cada $n, H_{n}$ tem uma filtração da forma

$$
0=F_{-1} H_{n} \subseteq F_{0} H_{n} \subseteq \cdots \subseteq F_{n-1} H_{n} \subseteq F_{n} H_{n}=H_{n},
$$

tal que $E_{p, n-p}^{\infty}=\frac{F_{p} H_{n}}{F_{p-1} H_{n}}$. Note que $E_{0, n}^{\infty} \cong F_{0} H_{n}$ é um quociente de $E_{0, n}^{a}$ e $E_{n, 0}^{\infty} \cong \frac{H_{n}}{F_{n-1} H_{n}}$ é um submódulo de $E_{n, 0}^{a}$. Os homomorfismos $E_{0, n}^{a} \rightarrow E_{0, n}^{\infty}$ e $E_{n, 0}^{\infty} \hookrightarrow E_{n, 0}^{a}$ são chamados os homomorfismos bordos. 
Sejam $E_{p, q}^{r}$ e $\mathscr{E}_{p, q}^{r}$ sequências espectrais com diferenciais $d_{p, q}^{r}$ e $\bar{d}_{p, q}^{r}$ respetivamente. Um morfismo entre estas sequências espectrais é uma família de aplicações $\left\{f_{p, q}^{r}: E_{p, q}^{r} \rightarrow \mathscr{E}_{p, q}^{r}\right\}_{p, q \in \mathbb{Z}}$ tais que

i) para todo $p, q \in \mathbb{Z}$ e $r \in \mathbb{Z}_{\geq 0}, f_{p-r, q+r-1}^{r} \circ d_{p, q}^{r}=\bar{d}_{p, q}^{r} \circ f_{p, q}^{r}$.

ii) para todo $p, q \in \mathbb{Z}$ e $r \in \mathbb{Z}_{\geq 0}, f_{p, q}^{r+1}$ é dada por

$$
\begin{aligned}
f_{p, q}^{r+1}: E_{p, q}^{r+1} & \longrightarrow \mathscr{E}_{p, q}^{r+1} . \\
x+\operatorname{Im} d_{p+r, q-r+1}^{r} & \longmapsto f_{p, q}^{r}(x)+\operatorname{Im} \bar{d}_{p+r, q-r+1}^{r}
\end{aligned}
$$

Nas sequências espectrais limitadas com $E_{p, q}^{\infty}=E_{p, q}^{r_{0}}$, definimos

$$
\begin{aligned}
Z_{p, q}^{\infty}=Z_{p, q}^{r_{0}} & :=\operatorname{Ker} d_{p, q}^{r_{0}}, \\
B_{p, q}^{\infty}=B_{p, q}^{r_{0}} & :=\operatorname{Im} d_{p+r_{0}, q-r_{0}+1}^{r_{0}} .
\end{aligned}
$$

Seja $\left\{f_{p, q}: E_{p, q}^{r} \rightarrow \mathscr{E}_{p, q}^{r}\right\}_{p, q \in \mathbb{Z}}$ um morfismo de sequências espectrais limitadas. Suponha que para algum $r_{0} \in \mathbb{N}$ fixo, $f_{p, q}^{r}: E_{p, q}^{r} \rightarrow \mathscr{E}_{p, q}^{r}$ é um isomorfismo para todo $p, q \in \mathbb{Z}$. Então para $s \geq r_{0}$ temos que $f_{p, q}^{s}: E_{p, q}^{s} \rightarrow \mathscr{E}_{p, q}^{s}$ é um isomorfismo. Definimos para todo $p, q \in \mathbb{Z}$ o homomorfismo $f_{p, q}^{\infty}: E_{p, q}^{\infty} \rightarrow \mathscr{E}_{p, q}^{\infty}$ como sendo $f_{p, q}^{\infty}\left(x+B_{p, q}^{\infty}\right)=f_{p, q}^{r_{0}}(x)+\mathscr{B}_{p, q}^{\infty}$, onde $\mathscr{E}_{p, q}^{\infty}=\frac{\mathscr{Z}_{p, q}^{\infty}}{\mathscr{B}_{p, q}^{\infty}}$. Note que $f_{p, q}^{\infty}$ é um isomorfismo.

Sejam $E_{p, q}^{a} \Longrightarrow H_{p+q}$ e $\mathscr{E}_{p, q}^{a} \Longrightarrow H_{p+q}^{\prime}$ sequências espectrais limitadas. Dizemos que uma família de homomorfismos $h_{\bullet}: H_{\bullet} \rightarrow H_{\bullet}^{\prime}$ é compatível com um morfismo de sequências espectrais $\left\{f_{p, q}: E_{p, q}^{r} \rightarrow \mathscr{E}_{p, q}^{r}\right\}_{p, q \in \mathbb{Z}}$ se

i) para todo $p \in \mathbb{Z}, h_{p}\left(F_{p} H_{n}\right) \subseteq F_{p}^{\prime} H_{n}^{\prime}$,

ii) para todo $p, n \in \mathbb{Z}$, se $E_{p, n-p}^{\infty} \stackrel{\beta_{p, n-p}}{\cong} \frac{F_{p} H_{n}}{F_{p-1} H_{n}}$ e $\mathscr{E}_{p, n-p} \stackrel{\beta_{p, n-p}^{\prime}}{\cong} \frac{F_{p}^{\prime} H_{n}^{\prime}}{F_{p-1}^{\prime} H_{n}^{\prime}}$, então temos um morfismo bem definido $\bar{h}_{p}: \frac{F_{p} H_{n}}{F_{p-1} H_{n}} \rightarrow \frac{F_{p}^{\prime} H_{n}^{\prime}}{F_{p-1}^{\prime} H_{n}^{\prime}}$ tal que

$$
f_{p, n-p}^{\infty} \circ \beta_{p, n-p}=\beta_{p, n-p}^{\prime} \circ \bar{h}_{p} .
$$

Neste caso escrevemos

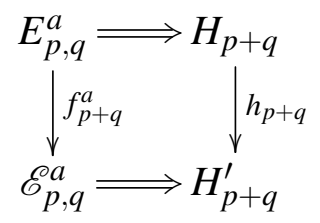

TEOREMA 1.11 (Teorema da comparação de Sequências Espectrais). Sejam $E_{p, q}^{a} \Rightarrow H_{p+q} e$ $\mathscr{E}_{p, q}^{a} \Rightarrow H_{p+q}^{\prime}$ sequências espectrais do primeiro quadrante. Suponha dada uma família $h_{\bullet}$ : $H_{\bullet} \rightarrow H_{\bullet}^{\prime}$ compativel com um morfismo de sequências espectrais $\left\{f_{p, q}: E_{p, q}^{r} \rightarrow \mathscr{E}_{p, q}^{r}\right\}_{p, q \in \mathbb{Z}}$. Se $f_{p, q}^{r}: E_{p, q}^{r} \rightarrow \mathscr{E}_{p, q}^{r}$ é um isomorfismo para $r \in \mathbb{N}$ fixo e para todo $p, q \in \mathbb{Z}$, então $h_{n}$ é um isomorfismo, para todo $n$. 
Demonstração. Sabemos que $H_{n}$ e $H_{n}^{\prime}$ têm filtrações

$$
\begin{aligned}
& 0=F_{-1} H_{n} \subseteq F_{0} H_{n} \subseteq \cdots \subseteq F_{n-1} H_{n} \subseteq F_{n} H_{n}=H_{n}, \\
& 0=F_{-1}^{\prime} H_{n}^{\prime} \subseteq F_{0}^{\prime} H_{n}^{\prime} \subseteq \cdots \subseteq F_{n-1}^{\prime} H_{n}^{\prime} \subseteq F_{n}^{\prime} H_{n}^{\prime}=H_{n}^{\prime},
\end{aligned}
$$

tais que $h_{n}\left(F_{p} H_{n}\right) \subseteq F_{p}^{\prime} H_{n}^{\prime} \mathrm{e}$

$$
E_{p, n-p}^{\infty} \cong \frac{F_{p} H_{n}}{F_{p-1} H_{n}}, \quad \mathscr{E}_{p, n-p}^{\infty} \cong \frac{F_{p}^{\prime} H_{n}^{\prime}}{F_{p-1}^{\prime} H^{\prime} n} .
$$

Como $f_{p, n-p}^{r}$ é um isomorfismo, $E_{p, n-p}^{\infty} \cong \mathscr{E}_{p, n-p}^{\infty}$. Temos assim o seguinte diagrama comutativo com linhas exatas

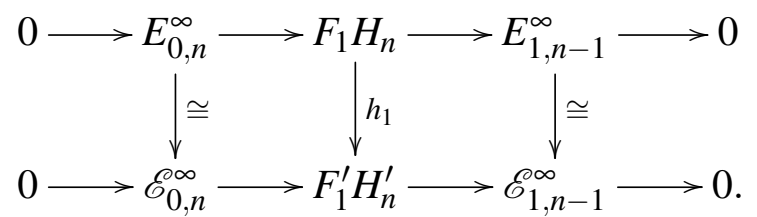

Logo, pelo Lema da serpente, $F_{1} H_{n} \cong F_{1}^{\prime} H_{n}^{\prime}$. Fazendo o mesmo tratamento para

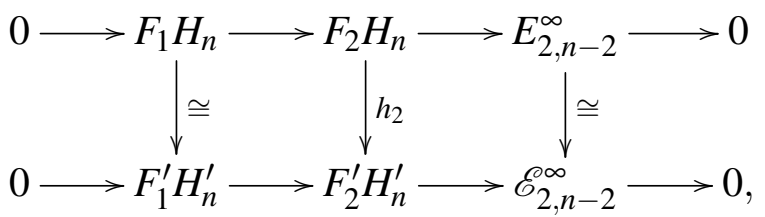

temos que $F_{2} H_{n} \cong F_{2}^{\prime} H_{n}^{\prime}$. Continuando este processo obtemos $H_{n}=F_{n} H_{n} \cong F_{n}^{\prime} H_{n}^{\prime}=H_{n}^{\prime}$.

Uma filtração $F$ sobre um complexo de cadeias $\left(C_{\bullet}, d_{\bullet}\right)$ é uma família de subcomplexos

$$
\cdots \subseteq\left(F_{p-1} C_{\bullet}, d_{\bullet}\right) \subseteq\left(F_{p} C_{\bullet}, d_{\bullet}\right) \subseteq\left(F_{p+1} C_{\bullet}, d_{\bullet}\right) \subseteq \cdots \subseteq\left(C_{\bullet}, d_{\bullet}\right)
$$

Dizemos que esta filtração de $\left(C_{\bullet}, d_{\bullet}\right)$ é limitada se para cada $n$, existem números inteiros $s \leq t$ tais que $F_{s} C_{n}=0$ e $F_{t} C_{n}=0$.

Teorema 1.12 (Teorema da Convergência Clásico). Se a filtração do complexo $\left(C_{\bullet}, d_{\bullet}\right)$ é limitada, então existe uma sequência espetral limitada

$$
E_{p, q}^{1}=H_{p+q}\left(\frac{F_{p} C_{\bullet}}{F_{p-1} C_{\bullet}}\right) \Longrightarrow H_{p+q}\left(C_{\bullet}\right)
$$

Além disso esta convergência é natural, isto é, se $f_{\bullet}:\left(C_{\bullet}, d_{\bullet}\right) \rightarrow\left(C_{\bullet}^{\prime}, d_{\bullet}^{\prime}\right)$ é um morfismo de complexos com filtrações limitadas, então $f_{*}: H_{*}\left(C_{\bullet}\right) \rightarrow H_{*}\left(C_{\bullet}^{\prime}\right)$ é compatível com o morfismo de sequências espectrais $E_{p, q}^{r} \rightarrow \mathscr{E}_{p, q}^{r}$ induzido por $f_{\bullet}$. 
Exemplo 1.27. Dizemos que uma filtração $F$ sobre $\left(C_{\bullet}, d_{\bullet}\right)$ é canonicamente limitada, se para todo $n, F_{-1} C_{n}=0$ e $F_{n} C_{n}=C_{n}$. Esta filtração gera uma sequência espectral com $E_{p, q}^{0}:=$ $\frac{F_{p} C_{p+q}}{F_{p-1} C_{p+q}}$. Note que se $p<0$ ou $q<0$, então $E_{p, q}^{0}=0$. Portanto temos uma sequência espectral do primeiro quadrante

$$
E_{p, q}^{1}=H_{p+q}\left(\frac{F_{p} C_{\bullet}}{F_{p-1} C_{\bullet}}\right) \Longrightarrow H_{p+q}\left(C_{\bullet}\right)
$$

Sejam $\left(C_{\bullet}, d_{\bullet}\right)$ e $\left(C_{\bullet}^{\prime}, d_{\bullet}^{\prime}\right)$ complexos de cadeias a esquerda de $R$-módulos. Considere $\left(D_{\bullet}, \partial_{\bullet}\right)$ como sendo o complexo produto de $C_{\bullet} \otimes C_{\bullet}^{\prime}$ definido em (1.1). Definamos as seguintes filtrações sobre $\left(D_{\bullet}, \partial_{\bullet}\right)$

$$
\begin{gathered}
{ }^{I} F_{n} D_{k}:=\bigoplus_{\substack{p+q=k \\
p \leq n \\
{ }^{I} F_{n} D_{k}}} C_{p} \otimes_{R} C_{q}^{\prime}, \\
\bigoplus_{\substack{p+q=k \\
q \leq n}} C_{p} \otimes_{R} C_{q}^{\prime} .
\end{gathered}
$$

Claramente estas duas filtrações são canonicamente limitadas. Logo pelo Teorema (1.12) e pelo Exemplo (1.27) temos duas sequências espectrais no primeiro quadrante

$$
{ }^{I} E_{p, q}^{1}=H_{p+q}\left(\frac{{ }^{I} F_{p} D_{\bullet}}{{ }^{I} F_{p-1} D_{\bullet}}\right) \Longrightarrow H_{p+q}\left(D_{\bullet}\right), \quad{ }^{I I} E_{p, q}^{1}=H_{p+q}\left(\frac{{ }^{I I} F_{p} D_{\bullet}}{{ }^{I I} F_{p-1} D_{\bullet}}\right) \Longrightarrow H_{p+q}\left(D_{\bullet}\right)
$$

onde

$$
\begin{gathered}
{ }^{I} E_{p, q}^{0}=\frac{{ }^{I} F_{p} D_{p+q}}{{ }^{I} F_{p-1} D_{p+q}}=\bigoplus_{\substack{i+j=p+q \\
i \leq p}} C_{i} \otimes_{R} C^{\prime}{ }_{j} / \bigoplus_{\substack{i^{\prime}+j^{\prime}=p+q \\
i^{\prime} \leq p-1}} C_{i^{\prime}} \otimes_{R} C_{j^{\prime}}^{\prime}=C_{p} \otimes_{R} C_{q}^{\prime}, \\
{ }^{I I} E_{p, q}^{0}=\frac{{ }^{I I} F_{p} D_{p+q}}{{ }^{I I} F_{p-1} D_{p+q}}=\bigoplus_{\substack{i+j=p+q \\
j \leq p}} C_{i} \otimes_{R} C^{\prime}{ }_{j} / \bigoplus_{\substack{i^{\prime}+j^{\prime}=p+q \\
j^{\prime} \leq p-1}} C_{i^{\prime}} \otimes_{R} C_{j^{\prime}}^{\prime}=C_{q} \otimes_{R} C_{p}^{\prime} .
\end{gathered}
$$

Note que ${ }^{I} E_{p, q}^{1}$ é a homologia na $(p+q)$-ésima posição do complexo $\frac{{ }^{I} F_{p} D_{\bullet}}{{ }^{I} F_{p-1} D_{\bullet}}$, isto é, a homologia de

$$
C_{p} \otimes_{R} C_{\bullet}^{\prime}: \cdots \rightarrow C_{p} \otimes_{R} C_{n-p}^{\prime} \rightarrow \cdots \rightarrow C_{p} \otimes_{R} C_{p-q}^{\prime} \rightarrow \cdots \rightarrow C_{p} \otimes_{R} C_{1-p}^{\prime} \rightarrow C_{p} \otimes_{R} C_{-p}^{\prime} \rightarrow \cdots
$$

na posição $p+q$. Assim, ${ }^{I} E_{p, q}^{1}=H_{q}\left(C_{p} \otimes_{R} C_{\bullet}^{\prime}\right)$ e os diferenciais são dados por

$$
{ }^{I} d_{p, q}^{1}=H_{q}\left(d_{p} \otimes i d_{C_{\bullet}^{\prime}}\right): H_{q}\left(C_{p} \otimes_{R} C_{\bullet}^{\prime}\right) \rightarrow H_{q}\left(C_{p-1} \otimes_{R} C_{\bullet}^{\prime}\right)
$$

Analogamente, ${ }^{I I} E_{p, q}^{1}=H_{q}\left(C \bullet \otimes_{R} C_{p}^{\prime}\right) \mathrm{e}$

$$
{ }^{I I} d_{p, q}^{1}=H_{q}\left(i d_{C \bullet} \otimes d_{p}^{\prime}\right): H_{q}\left(C_{\bullet} \otimes_{R} C_{p}^{\prime}\right) \rightarrow H_{q}\left(C_{\bullet} \otimes_{R} C_{p-1}^{\prime}\right) .
$$

Reescrevemos esse resultado no seguinte teorema. 
TEOREMA 1.13. Se $\left(C_{\bullet}, d_{\bullet}\right) e\left(C_{\bullet}^{\prime}, d_{\bullet}^{\prime}\right)$ são complexos à esquerda de R-módulos, então temos as seguintes sequências espectrais

$$
\begin{aligned}
& { }^{I} E_{p, q}^{1}=H_{q}\left(C_{p} \otimes_{R} C_{\bullet}^{\prime}\right) \Longrightarrow H_{p+q}\left(C_{\bullet} \otimes_{R} C_{\bullet}^{\prime}\right), \\
& { }^{I I} E_{p, q}^{1}=H_{q}\left(C_{\bullet} \otimes_{R} C_{p}^{\prime}\right) \Longrightarrow H_{p+q}\left(C_{\bullet} \otimes_{R} C_{\bullet}^{\prime}\right) \text {, }
\end{aligned}
$$

onde $^{I} d_{p, q}^{1}=H_{q}\left(d_{p} \otimes i d_{C_{\bullet}^{\prime}}\right) e^{I I} d_{p, q}^{1}=H_{q}\left(i d_{C_{\bullet}} \otimes d_{p}^{\prime}\right)$.

Seja $G$ um grupo e seja $\left(C_{\bullet}, d_{\bullet}\right)$ um complexo de $G$-módulos. Para $n \geq 0$, definimos o $n$-ésimo grupo de homologia de $G$ com coeficientes no complexo $C$ • como

$$
H_{n}\left(G, C_{\bullet}\right):=H_{n}\left(P_{\bullet} \otimes_{G} C_{\bullet}\right),
$$

onde $P_{\bullet} \rightarrow \mathbb{Z}$ é uma resolução projetiva de $\mathbb{Z}$ sobre $G$. Note que se $M$ é um $G$-módulo, então

$$
H_{n}\left(G, M_{\bullet}\right)=H_{n}(G, M)
$$

onde $M_{\bullet}$ é definido como no Exemplo (1.1). Definimos o complexo augmentado de $\left(C_{\bullet}, d_{\bullet}\right)$ por $M$ como

$$
\cdots \rightarrow C_{n} \stackrel{d_{n}}{\rightarrow} C_{n-1} \rightarrow \cdots \rightarrow C_{1} \rightarrow C_{0} \rightarrow M \rightarrow 0,
$$

e denotamos na forma $C_{\bullet} \rightarrow M$.

TEOrema 1.14. Seja $G$ um grupo e seja $C_{\bullet} \rightarrow M$ um complexo de G-módulos. Então existe uma sequência espectral no primeiro quadrante

$$
E_{p, q}^{1}=H_{q}\left(G, C_{p}\right) \Longrightarrow H_{p+q}\left(G, C_{\bullet}\right)
$$

tal que $d_{p, q}^{1}=H_{q}\left(d_{p+1}\right)$. Se o complexo $C_{\bullet} \rightarrow M$ é exato, então para todo $n, H_{n}\left(G, C_{\bullet}\right) \cong$ $H_{n}(G, M)$. Portanto

$$
E_{p, q}^{1}=H_{q}\left(G, C_{p}\right) \Longrightarrow H_{p+q}(G, M)
$$

Demonstração. Seja $P_{\bullet} \rightarrow \mathbb{Z}$ uma resolução projetiva de $\mathbb{Z}$ sobre $G$. Então pelo Teorema (1.13), temos duas sequências espectrais

$$
\begin{aligned}
& { }^{I} E_{p, q}^{1}=H_{q}\left(P_{p} \otimes_{G} C_{\bullet}\right) \Longrightarrow H_{p+q}\left(P_{\bullet} \otimes_{G} C_{\bullet}\right)=H_{p+q}\left(G, C_{\bullet}\right), \\
& { }^{I I} E_{p, q}^{1}=H_{q}\left(P_{\bullet} \otimes_{G} C_{p}\right) \Longrightarrow H_{p+q}\left(P_{\bullet} \otimes{ }_{G} C_{\bullet}\right)=H_{p+q}\left(G, C_{\bullet}\right) .
\end{aligned}
$$

Pela definição de homologia de grupos

$$
{ }^{I I} E_{p, q}^{1}=H_{q}\left(P \bullet \otimes_{G} C_{p}\right)=H_{q}\left(G, C_{p}\right) .
$$

Se $E_{p, q}^{1}:={ }^{I I} E_{p, q}^{1}$, então temos a sequência espectral

$$
E_{p, q}^{1}=H_{q}\left(G, C_{p}\right) \Longrightarrow H_{p+q}\left(G, C_{\bullet}\right),
$$


onde $d_{p, q}^{1}=H_{q}\left(d_{p+1}\right)$. Agora, seja $C_{\bullet} \rightarrow M$ um complexo exato. Como $P_{p}$ é projetivo, então o complexo $P_{p} \otimes_{G} C_{\bullet} \rightarrow P_{p} \otimes_{G} M$ é exato e logo

$$
{ }^{I} E_{p, q}^{1}= \begin{cases}P_{p} \otimes_{G} M & \text { se } q=0 \\ 0 & \text { se } q \neq 0\end{cases}
$$

Portanto

$$
{ }^{I} E_{p, q}^{2}=\left\{\begin{array}{ll}
H_{p}\left(P_{\bullet} \otimes_{G} M\right) & \text { se } q=0 \\
0 & \text { se } q \neq 0
\end{array}= \begin{cases}H_{p}(G, M) & \text { se } q=0, \\
0 & \text { se } q \neq 0 .\end{cases}\right.
$$

Pelo Exemplo (1.25), a sequência espectral ${ }^{I} E_{p, q}^{1} \Longrightarrow H_{p+q}\left(G, C_{\bullet}\right)$ implica que $H_{n}\left(G, C_{\bullet}\right) \cong$ $H_{n}(G, M)$ para todo $n$.

Seja $G$ um grupo e $H$ um subgrupo normal de $G$. Se $M$ é um $G$-módulo, então $M_{H}$ é um $H$-módulo trivial. Portanto $M_{H}$ é um $G / H$-módulo. Agora, em $\left(M_{H}\right)_{G / H}$

$$
g H(m+N)-(m+N)=(g m-m)+N
$$

para todo $m \in M$ e para todo $g \in G$, onde $N$ é o $H$-módulo gerado pelos elementos $h m-m$, $h \in H$. Logo, $\left(M_{H}\right)_{G / H}=M_{G}$.

TEOREMA 1.15 (Sequência Espectral de Lyndon/Hochschild-Serre). Para todo subgrupo normal $H$ do grupo $G$ e todo G-módulo $M$, existe uma sequência espectral do primeiro quadrante

$$
E_{p, q}^{2}=H_{p}\left(G / H, H_{q}(H, M)\right) \Longrightarrow H_{p+q}(G, M)
$$

Além disso, esta convêrgencia é natural. Esta sequência espectral implica a sequência exata

$$
H_{2}(G, M) \longrightarrow H_{2}\left(G / H, M_{H}\right) \longrightarrow H_{1}(G, M)_{G / H} \longrightarrow H_{1}(G, M) \longrightarrow H_{1}\left(G / H, M_{H}\right) \longrightarrow 0
$$

Demonstração. Seja $P \bullet \rightarrow \mathbb{Z}$ uma resolução projetiva de $\mathbb{Z}$ sobre $G$. Considere o complexo $C$ • de $G / H-$ módulos definido por $C_{p}:=P_{p} \otimes_{H} M \cong\left(P_{p} \otimes_{\mathbb{Z}} M\right)_{H}$. Seja $P_{\bullet}^{\prime} \rightarrow \mathbb{Z}$ uma resolução projetiva de $\mathbb{Z}$ sobre $G / H$. Pelo Teorema (1.13) temos duas sequências espectrais

$$
\begin{aligned}
& { }^{I} E_{p, q}^{1}=H_{q}\left(P_{p}^{\prime} \otimes_{G / H} C_{\bullet}\right) \Longrightarrow H_{p+q}\left(G / H, C_{\bullet}\right), \\
& { }^{I I} E_{p, q}^{1}=H_{q}\left(P_{\bullet}^{\prime} \otimes_{G / H} C_{p}\right) \Longrightarrow H_{p+q}\left(G / H, C_{\bullet}\right) \text {, }
\end{aligned}
$$

Como $P_{p}^{\prime}$ é um $G / H$-módulo projetivo, o funtor $P_{p}^{\prime} \otimes_{G / H}-$ é exato. $\log 0$

$$
{ }^{I} E_{p, q}^{1}=H_{q}\left(P_{p}^{\prime} \otimes_{G / H} C_{\bullet}\right) \cong P_{p}^{\prime} \otimes_{G / H} H_{q}\left(C_{\bullet}\right) \cong P_{p}^{\prime} \otimes_{G / H} H_{q}\left(P_{\bullet} \otimes_{H} M\right) \cong P_{p}^{\prime} \otimes_{G / H} H_{q}(H, M) .
$$

Tomando a homologia sobre $p$ temos

$$
{ }^{I} E_{p, q}^{2}=H_{p}\left(G / H, H_{q}(H, M)\right) \Longrightarrow H_{p+q}\left(G / H, C_{\bullet}\right)
$$


onde a ação de $G / H$ é definida como no Exemplo (1.18). Agora na outra sequência espectral temos

$$
{ }^{I I} E_{p, q}^{1}= \begin{cases}\left(C_{p}\right)_{G / H} & \text { se } q=0 \\ 0 & \text { se } q \neq 0\end{cases}
$$

Dado que $\left(C_{p}\right)_{G / H}=\left(\left(P_{p} \otimes M\right)_{H}\right)_{G / H}=\left(P_{p} \otimes M\right)_{G}=P_{p} \otimes_{G} M$, temos

$$
{ }^{I I} E_{p, q}^{2}=\left\{\begin{array}{ll}
H_{p}\left(P \bullet \otimes_{G} M\right) & \text { se } q=0 \\
0 & \text { se } q \neq 0
\end{array}= \begin{cases}H_{p}(G, M) & \text { se } q=0, \\
0 & \text { se } q \neq 0 .\end{cases}\right.
$$

Pelo Exemplo (1.25), a sequência espectral ${ }^{I I} E_{p, q}^{2} \Longrightarrow H_{p+q}\left(G / H, C_{\bullet}\right)$ implica que $H_{n}\left(G / H, C_{\bullet}\right) \cong$ $H_{n}(G, M)$ para todo $n$. Portanto se $E_{p, q}^{2}:={ }^{I} E_{p, q}^{2}$, então

$$
E_{p, q}^{2}=H_{p}\left(G / H, H_{q}(H, M)\right) \Longrightarrow H_{p+q}(G, M)
$$

Agora essa sequência espectral induz uma filtração sobre $H_{1}(G, M)$ da forma

$$
0=F_{-1} H_{1}(G, M) \subseteq F_{0} H_{1}(G, M) \subseteq F_{1} H_{1}(G, M)=H_{1}(G, M)
$$

tal que

$$
\begin{aligned}
& E_{0,1}^{\infty} \cong \frac{F_{0} H_{1}(G, M)}{F_{-1} H_{1}(G, M)}=F_{0} H_{1}(G, M), \\
& E_{1,0}^{\infty} \cong \frac{F_{1} H_{1}(G, M)}{F_{0} H_{1}(G, M)}=\frac{H_{1}(G, M)}{F_{0} H_{1}(G, M)} .
\end{aligned}
$$

Assim temos a sequência exata

$$
0 \rightarrow E_{0,1}^{\infty} \rightarrow H_{1}(G, M) \rightarrow E_{1,0}^{\infty} \rightarrow 0
$$

Por outro lado, é fácil verificar que $E_{1,0}^{\infty}=E_{1,0}^{2}$ e $E_{0,1}^{\infty}=E_{0,1}^{3}=$ coker $d_{2,0}^{2}$, obtendo assim sequência exata

$$
0 \longrightarrow E_{2,0}^{\infty} \longrightarrow E_{2,0}^{2} \stackrel{d_{2,0}^{2}}{\longrightarrow} E_{0,1}^{2} \longrightarrow E_{0,1}^{\infty} \longrightarrow 0 .
$$

Juntando as duas sequências e dado que $E_{2,0}^{\infty}$ é um quociente de $H_{2}(G, M)$, temos a sequência exata

$$
H_{2}(G, M) \rightarrow H_{2}\left(G / H, H_{0}(H, M)\right) \rightarrow H_{0}\left(G / H, H_{1}(H, M)\right) \rightarrow H_{1}(G, M) \rightarrow H_{1}\left(G / H, H_{0}(H, M)\right) \rightarrow 0
$$

Portanto temos a sequência exata

$$
H_{2}(G, M) \rightarrow H_{2}\left(G / H, M_{H}\right) \rightarrow H_{1}(H, M)_{G / H} \rightarrow H_{1}(G, M) \rightarrow H_{1}\left(G / H, M_{H}\right) \rightarrow 0 .
$$

EXEMPLO 1.28. Seja $H$ um subgrupo normal de $G$ tal que a seguinte sequência exata de grupos cinde

$$
1 \rightarrow H \stackrel{f}{\longrightarrow} G \stackrel{g}{\longrightarrow} G / H \rightarrow 1,
$$


onde $\alpha: G / H \rightarrow G$ é uma seção de $g$. Considere o diagrama comutativo

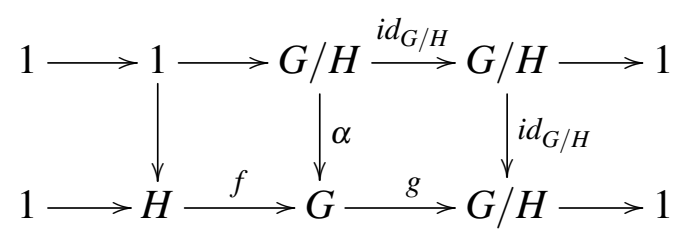

com linhas exatas. Pelo Teorema (1.15) temos um morfismo de sequências espectrais de Lyndon/Hochschild-Serre

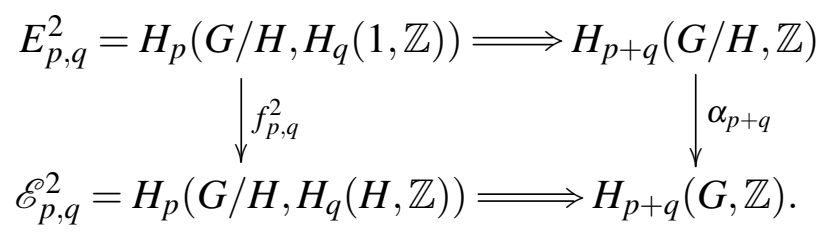

Como $H_{q}(1, \mathbb{Z})=0$ para todo $q \neq 0$ e $H_{0}(1, \mathbb{Z})=\mathbb{Z}$, temos que $E_{p, q}^{2}=0$ para todo $q \neq 0$ e $E_{p, 0}^{2}=H_{p}(G / H)$. Note que a aplicação $H_{0}(1) \rightarrow H_{0}(H)$ é um isomorfismo, $\operatorname{logo}$ para $r \geq 2$, temos que $f_{p, 0}^{r}: H_{p}(G / H) \rightarrow H_{p}(G / H)$ é um isomorfismo. Agora para $r \geq 2$ temos o seguinte diagrama comutativo

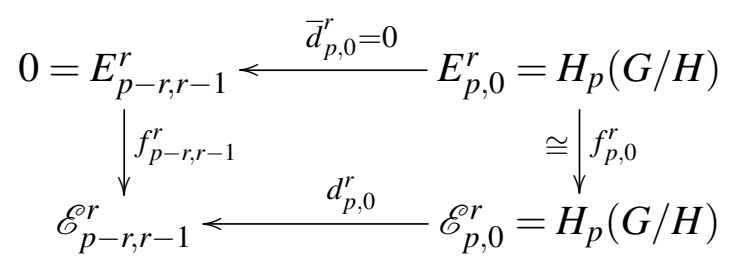

que implica que $d_{p, 0}^{r}=0$, para todo $r \geq 2$.

EXEMPLO 1.29. Seja $R$ um anel comutativo com unidade e considere $S L(R)$ como foi definido no Exemplo (1.19). Lembremos que se $A$ e $B$ são subgrupos de um grupo $G$, definimos $[A, B]$ como o subgrupo gerado por todos os elementos da forma $[a, b]:=a b a^{-1} b^{-1}$, onde $a \in A \mathrm{e}$ $b \in B$. Suponha que $R$ satisfaz que $S L(R)=[S L(R), S L(R)]$. A sequência exata de grupos

$$
1 \rightarrow S L(R) \stackrel{i}{\rightarrow} G L(R) \stackrel{\text { det }}{\rightarrow} R^{\times} \rightarrow 1,
$$

cinde pelo homomorfismo

$$
\phi: R^{\times} \longrightarrow G L(R), \quad x \longmapsto \operatorname{diag}(x, 1,1, \cdots) .
$$

Agora pelo Teorema (1.15) obtemos a sequência espectral de Lyndon/Hochschild-Serre

$$
\mathscr{E}_{p, q}^{2}=H_{p}\left(R^{\times}, H_{q}(S L(R))\right) \Longrightarrow H_{p+q}(G L(R))
$$

Assim temos que

$$
\begin{aligned}
& \mathscr{E}_{p, 0}^{2}=H_{p}\left(R^{\times}, H_{0}(S L(R))\right) \cong H_{p}\left(R^{\times}\right), \\
& \mathscr{E}_{p, 1}^{2}=H_{p}\left(R^{\times}, H_{1}(S L(R))\right) \cong H_{p}\left(R^{\times}, 0\right)=0,
\end{aligned}
$$


pois pelo Exemplo (1.10), $H_{1}(S L(R))=\frac{S L(R)}{[S L(R), S L(R)]}=0$. Por outro lado, pelo Exemplo (1.19), a ação de $R^{\times}$sobre $H_{2}(S L(R))$ é trivial, logo

$$
\mathscr{E}_{0,2}^{2}=H_{0}\left(R^{\times}, H_{2}(S L(R))\right) \cong H_{2}(S L(R))
$$

Assim a segunda folha desta sequência espectral é da forma

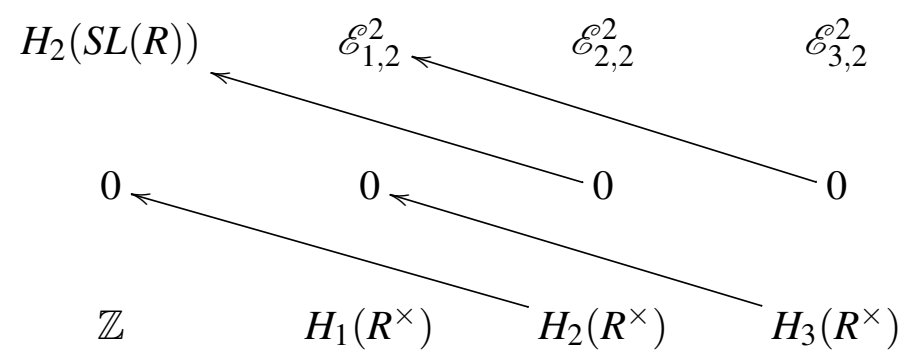

É fácil verificar que

$$
\mathscr{E}_{p, 0}^{3}=\frac{\operatorname{Ker} d_{p, 0}^{2}}{\operatorname{Im} d_{p+2,-1}^{2}}=\mathscr{E}_{p, 0}^{2}, \quad \mathscr{E}_{p, 1}^{3}=\frac{\operatorname{Ker} d_{p, 1}^{2}}{\operatorname{Im} d_{p+2,0}^{2}}=0
$$

e

$$
\mathscr{E}_{0,2}^{3}=\frac{\operatorname{Ker} d_{0,2}^{2}}{\operatorname{Im} d_{2,1}^{2}}=H_{2}(S L(R)) .
$$

Assim, a folha $E_{p, q}^{3}$ é da forma

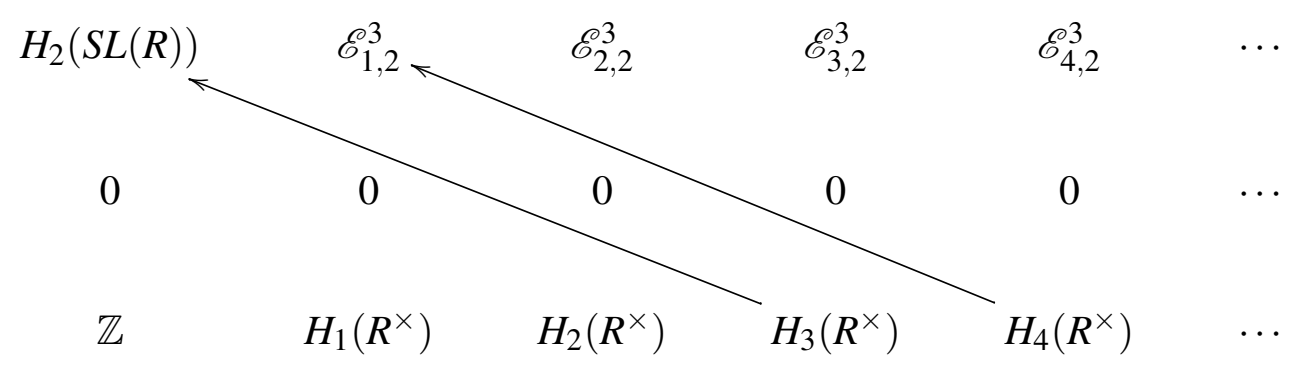

A sequência espectral $\mathscr{E}_{p, q}^{2}$ induz uma filtração sobre $H_{1}(G L(R))$ da forma

$$
0=F_{-1} H_{1}(G L(R)) \subseteq F_{0} H_{1}(G L(R)) \subseteq F_{1} H_{1}(G L(R))=H_{1}(G L(R)),
$$

de modo que

$$
\mathscr{E}_{0,1}^{\infty} \cong F_{0} H_{1}(G L(R)), \quad \mathscr{E}_{1,0} \cong \frac{H_{1}(G L(R))}{F_{0} H_{1}(G L(R))}
$$

isto implica

$$
H_{1}\left(R^{\times}\right) \cong H_{1}(G L(R)) .
$$

Note que pelo Exemplo (1.28), $d_{p, 0}^{3}=0$. Por outro lado, a sequência espectral $\mathscr{E}_{p, q}^{2}$ induz uma filtração sobre $H_{2}(G L(R))$ da forma

$$
0=F_{-1} H_{2}(G L(R)) \subseteq F_{0} H_{2}(G L(R)) \subseteq F_{1} H_{2}(G L(R)) \subseteq F_{2} H_{2}(G L(R))=H_{2}(G L(R)),
$$


de modo que

$$
\mathscr{E}_{0,2}^{\infty} \cong F_{0} H_{2}(G L(R)), \quad \mathscr{E}_{1,1}^{\infty} \cong \frac{F_{1} H_{2}(G L(R))}{F_{0} H_{2}(G L(R))}, \quad \mathscr{E}_{2,0}^{\infty} \cong \frac{H_{2}(G L(R))}{F_{1} H_{2}(G L(R))}
$$

Como $\mathscr{E}_{0,2}^{\infty} \cong H_{2}(S L(R)), \mathscr{E}_{1,1}^{\infty}=0$ e $\mathscr{E}_{2,0}^{\infty} \cong H_{2}\left(R^{\times}\right)$, obtemos a sequência exata

$$
0 \rightarrow H_{2}(S L(R)) \stackrel{i_{*}}{\longrightarrow} H_{2}(G L(R)) \stackrel{\operatorname{det}_{*}=\operatorname{cor}_{G L(R)}^{R^{\times}}}{\longrightarrow} H_{2}\left(R^{\times}\right) \rightarrow 0
$$

que cinde pelo homomorfismo induzido por $\phi: R^{\times} \rightarrow G L(R)$, pois $\operatorname{det}_{*} \circ \phi_{*}=(\operatorname{det} \circ \phi)_{*}=$ $\left(i d_{R^{\times}}\right)_{*}=i d_{H_{2}\left(R^{\times}\right)}$. Portanto temos a decomposição

$$
H_{2}(G L(R)) \cong H_{2}\left(R^{\times}\right) \oplus H_{2}(S L(R)) .
$$

Além disso, a sequência espectral $\mathscr{E}_{p, q}^{2}$ induz uma filtração sobre $H_{3}(G L(R))$ da forma

$$
0=F_{-1} H_{3}(G L(R)) \subseteq F_{0} H_{3}(G L(R)) \subseteq F_{1} H_{3}(G L(R)) \subseteq F_{2} H_{3}(G L(R)) \subseteq H_{3}(G L(R)),
$$

tal que

$$
\mathscr{E}_{0,3}^{\infty} \cong F_{0} H_{3}(G L(R)), \quad \mathscr{E}_{1,2}^{\infty} \cong \frac{F_{1} H_{3}(G L(R))}{F_{0} H_{3}(G L(R))}, \quad \mathscr{E}_{2,1}^{\infty} \cong \frac{F_{2} H_{3}(G L(R))}{F_{1} H_{3}(G L(R))}, \quad \mathscr{E}_{3,0}^{\infty} \cong \frac{H_{3}(G L(R))}{F_{2} H_{3}(G L(R))}
$$

É fácil verificar que $\mathscr{E}_{0,3}^{\infty} \cong H_{3}(S L(R)), \mathscr{E}_{2,1}^{\infty}=0$, e $\mathscr{E}_{3,0}^{\infty} \cong H_{3}\left(R^{\times}\right)$. Agora pelo Exemplo (1.14), temos que $\mathscr{E}_{1,2}^{\infty} \cong H_{1}\left(R^{\times}, H_{2}(S L(R))\right) \cong R^{\times} \otimes H_{2}(S L(R))$, obtendo assim as sequências exatas

$$
\begin{gathered}
0 \longrightarrow H_{3}(S L(R)) \longrightarrow F_{2} H_{3}(G L(R)) \longrightarrow R^{\times} \otimes H_{2}(S L(R)) \longrightarrow 0, \\
0 \longrightarrow F_{2} H_{3}(G L(R)) \longrightarrow H_{3}(G L(R)) \stackrel{\operatorname{det}_{*}}{\longrightarrow} H_{3}\left(R^{\times}\right) \longrightarrow 0 .
\end{gathered}
$$

Como $H_{3}(G L(R)) \cong F_{2} H_{3}(G L(R)) \oplus H_{3}\left(R^{\times}\right)$, temos a seguinte sequência exata

$$
0 \longrightarrow H_{3}(S L(R)) \longrightarrow \frac{H_{3}(G L(R))}{H_{3}\left(R^{\times}\right)} \longrightarrow R^{\times} \otimes H_{2}(S L(R)) \longrightarrow 0 .
$$

que cinde com uso do homomorfismo $\alpha_{*}: H_{3}(G L(R)) \rightarrow H_{3}(S L(R))$, onde $\alpha: G L(R) \rightarrow S L(R)$, $A \mapsto\left(\begin{array}{cc}(\operatorname{det} A)^{-1} & 0 \\ 0 & A\end{array}\right)$. Voltaremos nesse exemplo como parte da demonstração de nosso teorema principal no final do Capítulo 4.

\subsection{Homologia dos grupos lineares}

A continuação apresentaremos dois resultados muito importantes (sem demonstração) sobre homologia dos grupos lineares de ordem $n$. 
Proposição 1.4. Seja $R$ um anel local com ideal maximal $\mathfrak{m}$. Seja $G_{m}$ um subgrupo de $G L_{m}(R)$ e $G_{n}$ um subgrupo de $G L_{n}(R)$ tais que $R^{\times} I_{m} \subseteq G_{m}$ ou $R^{\times} I_{n} \subseteq G_{n}$. Seja $M(R)$ um submódulo de $M_{m, n}(R)$, tal que $G_{m} M(R)=M(R)=M(R) G_{n}$. Se $R / \mathfrak{m}$ é infinito, então a inclusão

$$
\left(\begin{array}{cc}
G_{m} & 0 \\
0 & G_{n}
\end{array}\right) \longrightarrow\left(\begin{array}{cc}
G_{m} & M(R) \\
0 & G_{n}
\end{array}\right)
$$

induz um isomorfismo de grupos

$$
H_{q}\left(G_{m} \times G_{n}\right) \stackrel{\cong}{\longrightarrow} H_{q}\left(\left(\begin{array}{cc}
G_{m} & M(R) \\
0 & G_{n}
\end{array}\right)\right)
$$

para todo $q \geq 0$.

Demonstração. Veja-se [11, Teorema 1.9].

EXEMPLO 1.30. Se $n=m=1$ e se $M(R)=R$, então para todo $q \geq 0$ temos o isomorfismo

$$
H_{q}\left(R^{\times} \times R^{\times}\right) \stackrel{\cong}{\longrightarrow} H_{q}\left(\left(\begin{array}{cc}
R^{\times} & R \\
0 & R^{\times}
\end{array}\right)\right) .
$$

TEOrema 1.16 (Teorema de Estabilidade Homológica para $G L_{n}(R)$ ). Seja $R$ um anel local com ideal maximal $\mathfrak{m}$. Se $R / \mathfrak{m}$ é um corpo infinito, então as inclusões

$$
G L_{n}(R) \longrightarrow G L_{n+1}(R), \quad A \longmapsto\left(\begin{array}{cc}
A & 0 \\
0 & 1
\end{array}\right)
$$

induzem os isomorfismos

$$
H_{n}\left(G L_{n}(R)\right) \stackrel{\cong}{\longrightarrow} H_{n}\left(G L_{n+1}(R)\right) \stackrel{\cong}{\longrightarrow} H_{n}\left(G L_{n+2}(R)\right) \stackrel{\cong}{\longrightarrow} H_{n}\left(G L_{n+3}(R)\right) \stackrel{\cong}{\longrightarrow} \cdots
$$

Em particular para todo $n, H_{n}\left(G L_{n}(R)\right) \stackrel{\cong}{\longrightarrow} H_{n}(G L(R))$. Além disso a inclusão

$$
i: R^{\times} \times G L_{n-1}(R) \longrightarrow G L_{n}(R), \quad(a, A) \longmapsto\left(\begin{array}{cc}
a & 0 \\
0 & A
\end{array}\right),
$$

induz o homomorfismo sobrejetor

$$
H_{n}\left(R^{\times} \times G L_{n-1}(R)\right) \stackrel{i_{*}=H_{n}(i)}{\longrightarrow} H_{n}\left(G L_{n}(R)\right) .
$$

Demonstração. Veja-se [7]. 



\section{$K_{2}$ DE ANÉIS E O TEOREMA DE} MATSUMOTO

A $K$-teoria algébrica associa para qualquer anel $R$ uma sequência de grupos abelianos

$K_{n}(R) n \geq 0$, chamados $K$-grupos de $R$. Neste capítulo estudaremos os $K$-grupos de ordem inferior de um anel comutativo com unidade $R$, a saber descreveremos os grupos $K_{0}(R), K_{1}(R)$ e $K_{2}(R)$. Como resultado principal, demonstraremos o teorema de Van der Kallen-Matsumoto sobre a estrutura do grupo $K_{2}(R)$ onde $R$ é um anel local com corpo residual infinito.

\section{$2.1 \quad K_{0}(R)$ e $K_{1}(R)$}

Para começar lembremos que um monoide abeliano é um conjunto $S$ munido com uma operação associativa e comutativa, denotada + , a qual tem elemento identidade $0 \in S$. Um exemplo de monoide abeliano é o conjunto dos números naturais $\mathbb{N}=\{0,1,2, \ldots\}$ com a adição usual.

Agora, para cada monoide abeliano $S$ associamos seu grupo completação, isto é um grupo abeliano $G$ junto com um homomorfismo de monoides $\phi: S \rightarrow G$ tal que para qualquer grupo abeliano $G^{\prime}$ e qualquer homomorfismo de monoides $\alpha: S \rightarrow G^{\prime}$ existe um único homomorfismo de grupos abelianos $\theta: G \rightarrow G^{\prime}$ tal que $\theta \circ \phi=\alpha$. Por exemplo, o grupo completação de $\mathbb{N}$ é o grupo dos inteiros $\mathbb{Z}$ com a adição usual.

Para todo monoide abeliano $S$ sempre podemos construir seu grupo completação como segue: Considere o grupo abeliano livre $\mathbb{Z}(S)$ gerado pelos símbolos $[s]$ com $s \in S$ e considere o grupo

$$
G=\frac{\mathbb{Z}(S)}{\left\langle\left[s+s^{\prime}\right]-[s]-\left[s^{\prime}\right]: s, s^{\prime} \in S\right\rangle},
$$

e defina o homomorfismo de monoides $\phi: S \rightarrow G$ dado por $s \rightarrow[s]$. É fácil verificar que $\phi: S \rightarrow G$ 
satisfaz a propriedade universal dada anteriormente.

DEFINIÇão 2.1. Seja $R$ um anel com unidade. Definimos o $K_{0}-$ grupo de $R$, denotado $K_{0}(R)$, como sendo o grupo completação de $\mathscr{P}(R)$

$$
K_{0}(R):=\frac{\mathbb{Z}(\mathscr{P}(R))}{\langle[P \oplus Q]-[P]-[Q]: P, Q \in \mathscr{P}(R)\rangle},
$$

onde $\mathscr{P}(R)$ é o monoide abeliano formado pelas classes de isomorfismo de R-módulos projetivos finitamente gerados $[P]$ junto com a soma direta $\oplus$ de $R$-módulos.

Note que em $K_{0}(R)$ temos a igualdade $[P \oplus Q]=[P]+[Q]$ onde $P$ e $Q$ são $R$-módulos projetivos finitamente gerados. Assim, $\left[R^{n}\right]=[R \oplus R \oplus \cdots \oplus R]=n[R] \in K_{0}(R)$ e $n[R]=m[R]$ se e somente se $n=m$ [5, Lema 1.1]. Isto permite descrever todo elemento em $K_{0}(R)$ como sendo $[P]-n[R]$ para algum $R$-módulo projetivo $P$ e algum $n \in \mathbb{N}$. Em geral, a descrição do grupo $K_{0}(R)$ é muito difícil, mas em alguns casos específicos temos uma melhor descrição.

Quando $R$ é um anel comutativo, no monoide $\mathscr{P}(R)$ temos que $P \otimes_{R} Q \cong Q \otimes_{R} P$. Além disso, se $P$ e $Q$ são $R$-módulos projetivos finitamente gerados, então também $P \otimes_{R} Q$ é um $R$-módulo projetivo finitamente gerado. Portanto $K_{0}(R)$ também é um anel comutativo com multiplicação $[P] \cdot[Q]:=\left[P \otimes_{R} Q\right]$ e $1_{K_{0}(R)}=[R]$.

Exemplo 2.1. Um $R$-módulo projetivo $P$ é dito estavelmente livre se existem $r, s \in \mathbb{N}$ tais que $P \oplus R^{r} \cong R^{s}$. Em $K_{0}(R)$ podemos dizer que esta caracterização é equivalente a dizer que $P=n[R]$ para algum $n \in \mathbb{N}$. Por exemplo se $R$ é um anel local ou um domínio de ideais principais, então os $R$-módulos projetivos finitamente gerados são livres [8, Teorema 1.3.1, Teorema 1.3.11] e portanto estávelmente livres. Assim temos um homomorfismo de grupos abelianos

$$
\varphi: K_{0}(R) \longrightarrow \mathbb{Z}, \quad[P] \longmapsto \operatorname{rank}_{R} P
$$

Dado que $\varphi([R])=1, \varphi$ é sobrejetor. Se $\varphi([P])=\varphi([Q])$, então $\operatorname{rank}_{R} P=\operatorname{rank}_{R} Q$. Assim $P \cong Q$, isto é $[P]=[Q]$, logo $\varphi$ é um isomorfismo. Em consequência, se $R$ é um anel local ou um domínio de ideais principais, então $K_{0}(R) \cong \mathbb{Z}$.

Seja $\varphi: R \rightarrow S$ um homomorfismo de anéis e seja $P$ um $R$-módulo projetivo finitamente gerado. Então existe um $R$-módulo $Q$ tal que $P \oplus Q \cong R^{n}$ para algum inteiro $n$, logo

$$
\left(P \otimes_{R} S\right) \oplus\left(Q \otimes_{R} S\right)=(P \oplus Q) \otimes_{R} S=R^{n} \otimes_{R} S=S^{n}
$$

Então o funtor

$$
-\otimes_{R} S:{ }_{R} \operatorname{Mod} \rightarrow{ }_{S} M o d \quad P \longmapsto P \otimes_{R} S
$$

induz um homomorfismo de monoides $\mathscr{P}(R) \rightarrow \mathscr{P}(S)$ e também induz um homomorfismo de grupos

$$
\varphi_{*}: K_{0}(R) \longrightarrow K_{0}(S), \quad[P] \longmapsto\left[P \otimes_{R} S\right]
$$


Trivialmente $\left(i d_{R}\right)_{*}=i d_{K_{0}(R)}$. Além disso, se $\psi: S \rightarrow T$ é outro homomorfismo de anéis, então $(\psi \circ \varphi)_{*}=\psi_{*} \circ \varphi_{*}$. Portanto $K_{0}(-)$ é um funtor da categoria de anéis na categoria de grupos abelianos. Assim, o homomorfismo natural de anéis $i: \mathbb{Z} \rightarrow R, n \rightarrow n .1$, induz um homomorfismo de grupos $i_{*}: \mathbb{Z} \rightarrow K_{0}(R), n \mapsto n[R]$. Se $R$ é um anel comutativo, o epimorfismo natural $\pi: R \rightarrow R / \mathfrak{m}, r \mapsto r+\mathfrak{m}$, onde $\mathfrak{m}$ é um ideal maximal de $R$, induz um homomorfismo de anéis $\pi_{*}: K_{0}(R) \rightarrow K_{0}(R / \mathfrak{m}) \cong \mathbb{Z},[P] \mapsto \operatorname{dim}_{R / \mathfrak{m}}(P / \mathfrak{m} P)$. Note que $\pi_{*}(n[R])=\pi_{*}\left(\left[R^{n}\right]\right)=$ $\operatorname{dim}_{R / \mathfrak{m}}\left(R^{n} / \mathfrak{m} R^{n}\right)=\operatorname{dim}_{R / \mathfrak{m}}(R / \mathfrak{m})^{n}=n, \log \mathrm{o} \pi_{*} \circ i_{*}(n)=\pi_{*}(n[R])=n$. Defina o grupo quociente $S K_{0}(R):=K_{0}(R) / \mathbb{Z}[R]$ chamado o $K_{0}$-grupo reduzido de $R$ e desta maneira temos que $K_{0}(R) \cong \mathbb{Z} \oplus S K_{0}(R)$.

DEFINIÇÃo 2.2. Seja $R$ um anel com unidade. Definimos o $K_{1}$-grupo de $R$, denotado $K_{1}(R)$, como sendo

$$
K_{1}(R):=\frac{G L(R)}{[G L(R), G L(R)]}
$$

Para todo $\lambda \in R$ e todo $i, j \in \mathbb{N}, 1 \leq i, j \leq n \operatorname{com} i \neq j$, definimos a matriz elementar $e_{i, j}^{n}(\lambda) \in$ $G L_{n}(R)$ como sendo a matriz com 1 na diagonal, $\lambda$ na posição $(i, j)$ e 0 nos outros lugares. $\mathrm{O}$ subgrupo de $G L_{n}(R)$ gerado pelas matrizes elementares é denotado $E_{n}(R)$.

Pela inclusão $G L_{n}(R) \subset G L_{n+1}(R)$, temos que $E_{n}(R) \subset E_{n+1}(R)$. A união infinita dos subgrupos $E_{n}(R)$ é denotada por $E(R)$ e chamada o grupo de matrizes elementares

$$
E(R):=\bigcup_{n \geq 1} E_{n}(R)
$$

Note que quando $R$ é comutativo, $E(R) \subset S L(R)$. Para $\lambda, \tau \in R$ temos

- se $i \neq j$, então $e_{i, j}^{n}(\lambda) e_{i, j}^{n}(\tau)=e_{i, j}^{n}(\lambda+\tau)$,

- se $j \neq k$ e $i \neq l$, então $e_{i, j}^{n}(\lambda) e_{k, l}^{n}(\tau)=e_{k, l}^{n}(\tau) e_{i, j}^{n}(\lambda)$,

- se $j=k$ e $i \neq l$, então $\left[e_{i, j}^{n}(\lambda), e_{k, l}^{n}(\tau)\right]=e_{i, l}^{n}(\lambda \tau)$,

- se $j \neq k$ e $i=l$, então $\left[e_{i, j}^{n}(\lambda), e_{k, l}^{n}(\tau)\right]=e_{k, j}^{n}(-\tau \lambda)$.

Portanto se $n \geq 3$, então $E_{n}(R)=\left[E_{n}(R), E_{n}(R)\right]$ e portanto $E(R)=[E(R), E(R)]$. Além disso, se $A \in G L_{n}(R)$ é uma matriz triangular superior (ou inferior) com 1 na diagonal, então $A \in E_{n}(R)$. Por exemplo para todo $B=\left(b_{i j}\right) \in G L_{n}(R)$

$$
\left(\begin{array}{cc}
I_{n} & B \\
0 & I_{n}
\end{array}\right)=\prod_{i, j=1}^{n} e_{n+i, j}^{2 n}\left(b_{i j}\right) \in E_{2 n}(R), \quad\left(\begin{array}{cc}
I_{n} & 0 \\
B & I_{n}
\end{array}\right)=\prod_{i, j=1}^{n} e_{i, n+j}^{2 n}\left(b_{i j}\right) \in E_{2 n}(R) .
$$

LEMA 2.1. Se $A \in G L_{n}(R)$, então $\left(\begin{array}{cc}A & 0 \\ 0 & A^{-1}\end{array}\right) \in E_{2 n}(R)$. 
Demonstração. Note que se $A \in G L_{n}(R)$, então

$$
\left(\begin{array}{cc}
0 & A \\
-A^{-1} & 0
\end{array}\right)=\left(\begin{array}{cc}
I_{n} & A \\
0 & I_{n}
\end{array}\right)\left(\begin{array}{cc}
I_{n} & 0 \\
-A^{-1} & I_{n}
\end{array}\right)\left(\begin{array}{cc}
I_{n} & A \\
0 & I_{n}
\end{array}\right) \in E_{2 n}(R)
$$

Em particular, $\left(\begin{array}{cc}0 & -I_{n} \\ I_{n} & 0\end{array}\right) \in E_{2 n}(R)$. Portanto

$$
\left(\begin{array}{cc}
A & 0 \\
0 & A^{-1}
\end{array}\right)=\left(\begin{array}{cc}
0 & A \\
-A^{-1} & 0
\end{array}\right)\left(\begin{array}{cc}
0 & -I_{n} \\
I_{n} & 0
\end{array}\right) \in E_{2 n}(R)
$$

Proposição 2.1 (Lema de Whitehead). Para todo anel com unidade $R$

$$
E(R)=[E(R), E(R)]=[G L(R), G L(R)]
$$

Demonstração. Já vimos que $E(R)=[E(R), E(R)], \log 0 E(R) \subseteq[G L(R), G L(R)]$. Se $A, B \in$ $G L_{n}(R)$, então pelo Lema (2.1)

$$
\left(\begin{array}{cc}
A B A^{-1} B^{-1} & 0 \\
0 & 1
\end{array}\right)=\left(\begin{array}{cc}
A B & 0 \\
0 & B^{-1} A^{-1}
\end{array}\right)\left(\begin{array}{cc}
A^{-1} & 0 \\
0 & A
\end{array}\right)\left(\begin{array}{cc}
B^{-1} & 0 \\
0 & B
\end{array}\right)
$$

Portanto $[G L(R), G L(R)] \subseteq E(R)$.

Se $R$ é um anel comutativo e $n \geq 3$, então $E_{n}(R)=\left[E_{n}(R), E_{n}(R)\right] \subseteq\left[G L_{n}(R), G L_{n}(R)\right] \subseteq S L_{n}(R)$. Assim, $E(R) \subseteq S L(R)$. Mas em geral a inclusão anterior nem sempre é uma igualdade. Defina o grupo quociente

$$
S K_{1}(R):=S L(R) / E(R),
$$

chamado o $K_{1}$-grupo reduzido de $R$. Por outro lado, o homomorfismo determinante, det $: G L(R) \rightarrow R^{\times}$, induz uma sobrejeção $\operatorname{det}_{*}: K_{1}(R) \rightarrow R^{\times}$, logo temos a sequência exata

$$
1 \rightarrow S K_{1}(R) \rightarrow K_{1}(R) \rightarrow R^{\times} \rightarrow 1
$$

Utilizando uma seção dada pela composição $R^{\times}=G L_{1}(R) \rightarrow G L(R) \rightarrow K_{1}(R)$ temos a decomposição

$$
K_{1}(R) \cong R^{\times} \oplus S K_{1}(R)
$$

Um vetor $v=\left(v_{1}, \ldots, v_{n}\right) \in R^{n}$ é dito unimodular se existe um vector $\left(x_{1}, \ldots, x_{n}\right) \in R^{n}$ tal que $\sum_{j=1}^{n} x_{j} v_{j}=1$. É fácil ver que $v=\left(v_{1}, \ldots, v_{n}\right) \in R^{n}$ é unimodular se e somente se $\left\langle v_{1}, \ldots, v_{n}\right\rangle=R$. Por exemplo, se $A=\left(a_{i j}\right) \in G L_{n}(R)$, usando o fato que $A A^{-1}=I_{n}$, temos que a $i$-ésima linha de $A,\left(a_{i 1}, a_{i 2}, \ldots, a_{i n}\right)$, é unimodular para todo $1 \leq i \leq n$. 
EXEMPLO 2.2. Seja $R$ um domínio euclidiano com aplicação norma ||$: R \rightarrow \mathbb{N}$. Vamos demonstrar que para todo $n \geq 2, \quad E_{n}(R)=S L_{n}(R)$. Claramente $E_{n}(R) \subseteq S L_{n}(R)$. Seja $A=\left(a_{i j}\right) \in S L_{n}(R)$ e seja $v=\left(a_{11}, \ldots, a_{n 1}\right) \in R^{n}$ a primeira coluna de $A$. Dado que $A \in G L_{n}(R)$ então não todos os elementos da primeira coluna podem ser nulos, logo existe $a_{k 1} \neq 0$ para algum $1 \leq k \leq n$. Escolha agora $a_{i 1} \in R$ tal que $\left|a_{i 1}\right|=\min \left\{\left|a_{11}\right|, \ldots,\left|a_{n 1}\right|\right\} . \operatorname{Se}\left|a_{i 1}\right|=1$, então pelo algoritmo euclidiano existem $q, r \in R$ tal que $1=q a_{i 1}+r \operatorname{com} 0 \leq|r|<\left|a_{i 1}\right|=1$. Isto implica que $r=0$ e portanto $a_{i 1} \in R^{\times}$. Por outro lado, se $\left|a_{i 1}\right|>1$, então $a_{i 1} \notin R^{\times} \mathrm{e}$ $\left\langle a_{i 1}\right\rangle \subsetneq R$. Logo existe $j \neq i$ tal que $a_{j 1} \notin\left\langle a_{i 1}\right\rangle$. Agora pelo algoritmo euclidiano existem $q_{j}, r_{j} \in R$ tal que $a_{j 1}=q_{j} a_{i 1}+r_{j} \operatorname{com} 0 \leq\left|r_{j}\right|<\left|a_{i 1}\right|$. Seja $A^{\prime}:=e_{j i}^{n}\left(-q_{j}\right) A \in S L_{n}(R)$, então $v^{\prime}=\left(v_{1}^{\prime}, \ldots, v_{n}^{\prime}\right)=\left(a_{11}, \ldots, a_{i 1}, \ldots, r_{j}, \ldots, a_{n 1}\right) \in R^{n}$ é a primeira coluna de $A^{\prime}$. Escolha novamente $v_{i}^{\prime} \in R$ tal que $\left|v_{i}^{\prime}\right|=\min \left\{\left|v_{1}^{\prime}\right|, \ldots,\left|v_{n}^{\prime}\right|\right\}<\left|a_{i 1}\right|$. Se $\left|v_{i}^{\prime}\right|=1$ então $v_{i}^{\prime} \in R^{\times}$e se $\left|v_{i}^{\prime}\right|>1$ fazendo o mesmo tratamento acima, podemos encontrar uma matriz $E \in E_{n}(R)$ tal que a primeira coluna $\left(w_{1}, \ldots, w_{n}\right)$ de $E A$ tem a propriedade que $\min \left\{\left|w_{1}\right|, \ldots,\left|w_{n}\right|\right\}<\left|v_{i}^{\prime}\right|$. Com este método podemos achar $E \in E_{n}(R)$ tal que a primeira coluna de $E A$ tenha um coeficiente $a_{i 1} \mathrm{em} R^{\times}$. Sem perda de generalidade podemos supor que $i=1$, isto é $a_{11} \in R^{\times}$, caso contrário consideramos $A^{\prime}=e_{1 i}^{n}(1) e_{i 1}^{n}(-1) e_{1 i}^{n}(1) A$, com isto obtemos que $a_{11}^{\prime} \in R^{\times}$. Assim, obtemos uma matriz em $S L_{n}(R)$ tal que a primeira coluna só tenha $a_{11} \in R^{\times}$e $a_{i 1}=0$ se $i \neq 1$. Desta maneira podemos encontrar matrizes $E, E^{\prime} \in E_{n}(R)$ tais que

$$
E A E^{\prime}=\left(\begin{array}{ccc}
r & 0 & 0 \\
0 & r^{-1} & 0 \\
0 & 0 & I_{n-2}
\end{array}\right)\left(\begin{array}{ll}
1 & 0 \\
0 & B
\end{array}\right)
$$

para algum $r \in R^{\times}$e $B \in S L_{n-1}(R)$. Fazendo indução sobre $n$, basta demonstrar que $\left(\begin{array}{cc}r & 0 \\ 0 & r^{-1}\end{array}\right) \in$ $E_{2}(R)$. Mas este é um caso especial do Lema (2.1). Portanto $S L_{n}(R) \subseteq E_{n}(R)$. Como consequência temos $S K_{1}(R)=1$ e logo $K_{1}(R) \cong R^{\times}$. Em particular, $K_{1}(\mathbb{Z}) \cong\{-1,+1\}$ e $K_{1}(F[x]) \cong F^{\times}$, onde $F$ é um corpo.

EXEMPLO 2.3. Seja $R$ um anel comutativo local com ideal maximal $\mathfrak{m}$. Vamos demonstrar que $E_{n}(R)=S L_{n}(R)$ para todo $n \geq 2$. Claramente $E_{n}(R) \subseteq S L_{n}(R)$ e de forma análoga como foi feito na última parte do exemplo anterior, podemos demonstrar que $S L_{n}(R) \subseteq E_{n}(R)$. Em consequência temos $S K_{1}(R)=1$ e portanto $K_{1}(R) \cong R^{\times}$.

Um homomorfismo de anéis $f: R \rightarrow S$ induz um homomorfismo de grupos $f_{n}: G L_{n}(R) \rightarrow$ $G L_{n}(S),\left(a_{i j}\right) \mapsto\left(f\left(a_{i j}\right)\right)$ que induz um homomorfismo de grupos $f_{\infty}: G L(R) \rightarrow G L(S)$. É claro que $f_{n}\left(E_{n}(R)\right) \subseteq E_{n}(S) \operatorname{logo} f_{\infty}(E(R)) \subseteq E(S)$. Assim obtemos um homomorfimos induzido de grupos abelianos

$$
K_{1}(f): K_{1}(R) \rightarrow K_{1}(S), \quad \overline{\left(a_{i j}\right)} \mapsto \overline{f\left(a_{i j}\right)}
$$

É fácil verificar que $K_{1}(-)$ é um funtor da categoria de anéis na categoria de grupos abelianos. 


\subsection{Extensões Universais}

Seja $G$ um grupo qualquer e seja $A$ um grupo abeliano. Uma extensão central de $G$ por $A$ é um par $(E, \varphi)$ onde $E$ é um grupo, $\varphi: E \rightarrow G$ é um homomorfismo sobrejetor de grupos tal que $A=\operatorname{Ker} \varphi \subseteq Z(E)$, onde $Z(E)$ é o centro de $E$. Normalmente denotamos esta extensão por

$$
1 \longrightarrow A \longrightarrow E \stackrel{\varphi}{\longrightarrow} G \longrightarrow 1 \text {. }
$$

Uma extensão central $(E, \varphi)$ de $G$ por $A$ cinde se existe um homomorfismo $s: G \rightarrow E$ tal que $\varphi \circ s=i d_{G}$. Uma extensão central $(E, \varphi)$ de $G$ por $A$ é dita universal se para qualquer outra extensão central $\left(E^{\prime}, \varphi^{\prime}\right)$ de $G$ por algum grupo abeliano $A^{\prime}$, existe um único homomorfismo $h: E \rightarrow E^{\prime}$ tal que $\varphi^{\prime} \circ h=\varphi$. Sejam $(E, \varphi),\left(E^{\prime}, \varphi^{\prime}\right)$ extensões centrais universais de $G$ por $A$ e $A^{\prime}$ respetivamente. Então existem homomorfismos $h: E \rightarrow E^{\prime}$ e $h^{\prime}: E^{\prime} \rightarrow E$ tais que $\varphi^{\prime} \circ h=\varphi$ e $\varphi \circ h^{\prime}=\varphi^{\prime}$. Portanto, $E \cong E^{\prime}$ e $A \cong A^{\prime}$.

Dizemos que um grupo $G$ é perfeito se $G=[G, G]$.

LEMA 2.2. Sejam $(E, \varphi)$ e $\left(E^{\prime}, \varphi^{\prime}\right)$ extensões centrais de G por A e $A^{\prime}$. Se E é perfeito, então só existe no máximo um homomorfismo de $h: E \rightarrow E^{\prime}$ tal que $\varphi^{\prime} \circ h=\varphi$.

Demonstração. Considere $h_{1}, h_{2}: E \rightarrow E^{\prime}$ homomorfismos tais que $\varphi^{\prime} \circ h_{1}=\varphi^{\prime} \circ h_{2}=\varphi$. Se $x, y \in E$, então

$$
\begin{aligned}
\varphi^{\prime}\left(h_{1}(x) h_{2}(x)^{-1}\right) & =\varphi(x) \varphi(x)^{-1}=1, \\
\varphi^{\prime}\left(h_{1}(y) h_{2}(y)^{-1}\right) & =\varphi(y) \varphi(y)^{-1}=1 .
\end{aligned}
$$

Logo existem $z, z^{\prime} \in A^{\prime} \subseteq Z\left(E^{\prime}\right)$ tais que $h_{1}(x)=h_{2}(x) z, h_{1}(y)=h_{2}(y) z^{\prime}$. Assim

$$
h_{1}\left(x y x^{-1} y^{-1}\right)=h_{1}(x) h_{1}(y) h_{1}(x)^{-1} h_{1}(y)^{-1}=h_{2}(x) h_{2}(y) h_{2}(x)^{-1} h_{2}(y)^{-1}=h_{2}\left(x y x^{-1} y^{-1}\right) .
$$

Dado que $h_{1}$ e $h_{2}$ coinciden em $[x, y]$ para todo $x, y \in E$ e já que $E$ é perfeito, então $h_{1}=h_{2}$.

LEMA 2.3. Seja $(E, \varphi)$ é uma extensão central de $G$ por $A$. Se $(E, \varphi)$ é universal, então $E$ é perfeito.

Demonstração. Suponha que $E$ não é perfeito. Considere $A^{\prime}=E /[E, E]$ e seja $\psi: E \rightarrow A^{\prime}$ o homomorfismo quociente. É claro que os homomorfismos

$$
\begin{aligned}
h_{1}: E \rightarrow G \times A^{\prime}, & x \longmapsto(\varphi(x), 1), \\
h_{2}: E \rightarrow G \times A^{\prime}, & x \longmapsto(\varphi(x), \psi(x)),
\end{aligned}
$$

são diferentes. Além disso, $\left(E^{\prime}=G \times A^{\prime}, \varphi^{\prime}\right)$ é uma extensão central de $G$ por $A^{\prime}$, onde $\varphi^{\prime}$ é a projeção de $G \times A^{\prime}$ sobre $G$, satisfazendo $\varphi^{\prime} \circ h_{1}=\varphi^{\prime} \circ h_{2}=\varphi$. Portanto, $(E, \varphi)$ não é universal.

LEMA 2.4. Seja $(E, \varphi)$ uma extensão central de G por A. Se G é perfeito, então o subgrupo comutador $E^{\prime}=[E, E]$ é perfeito e $\varphi\left(E^{\prime}\right)=G$. Portanto, $\left(E^{\prime},\left.\varphi\right|_{E^{\prime}}\right)$ é uma extensão central de $G$ por A. 
Demonstração. Se $g, g^{\prime} \in G$, então existem $x, x^{\prime} \in E$ tais que $\varphi(x)=g$ e $\varphi\left(x^{\prime}\right)=g^{\prime}$. Assim

$$
\varphi\left(\left[x, x^{\prime}\right]\right)=\varphi(x) \varphi\left(x^{\prime}\right) \varphi(x)^{-1} \varphi\left(x^{\prime}\right)^{-1}=\left[g, g^{\prime}\right] .
$$

Portanto $\varphi\left(E^{\prime}\right)=G$. Agora se $x \in E$ existe $x^{\prime} \in E^{\prime}$ tal que $\varphi(x)=\varphi\left(x^{\prime}\right)$. Logo $x=x^{\prime} z$ para algum $z \in \operatorname{Ker} \varphi \subseteq Z(E)$. Sejam $x=x^{\prime} z, y=y^{\prime} w \in E$, onde $z, w \in \operatorname{Ker} \varphi$. Então

$$
[x, y]=\left[x^{\prime} z, y^{\prime} w\right]=\left[x^{\prime}, y^{\prime}\right] \in\left[E^{\prime}, E^{\prime}\right]
$$

Portanto $E^{\prime}$ é perfeito.

Proposição 2.2. Uma extensão central $(E, \varphi)$ de G por A é universal se, e somente se, E é perfeito e toda extensão central de E cinde.

Demonstração. Seja $(E, \varphi)$ uma extensão central universal de $G$ por $A$. Pelo Lema (2.3), $E$ é perfeito. Seja $\left(E^{\prime \prime}, \psi\right)$ uma extensão central de $E$ por algum grupo abeliano $A^{\prime \prime}$. Defina $A^{\prime}:=$ $\operatorname{Ker}(\varphi \circ \psi)$. Se $x_{0} \in A^{\prime}$, então $\psi\left(x_{0}\right) \in A=\operatorname{Ker} \varphi \subset Z(E)$. Assim o homomorfismo

$$
h: E^{\prime \prime} \longrightarrow E^{\prime \prime}, \quad x \longmapsto x_{0} x x_{0}^{-1},
$$

satisfaz $\psi \circ h=\psi$. Se $X=\left[E^{\prime \prime}, E^{\prime \prime}\right]$, pelos Lemas (2.2) e (2.4) tem-se que $\left(X,\left.\psi\right|_{X}\right)$ é uma extensão central de $E$ e $\left.h\right|_{X}$ é a identidade. Portanto $x_{0}$ comuta com os elementos de $X$, pois $x=h(x)=x_{0} x x_{0}^{-1}$. Pelo Lema (2.4), se $x \in E^{\prime \prime}$, existem $x^{\prime} \in X$ e $z \in \operatorname{Ker} \psi \subseteq Z\left(E^{\prime \prime}\right)$ tais que $x=x^{\prime} z$. Logo $x_{0}$ comuta também com os elementos de $E^{\prime \prime}$. Portanto $A^{\prime} \subseteq Z\left(E^{\prime \prime}\right)$ e $\left(E^{\prime \prime}, \varphi \circ \psi\right)$ é uma extensão central de $G$ por $A^{\prime}$. Dado que $(E, \varphi)$ é universal existe um único homomorfismo $s: E \rightarrow E^{\prime \prime}$ tal que $\varphi=(\varphi \circ \psi) \circ s=\varphi \circ(\psi \circ s)$. Assim pelo Lema (2.2), $\psi \circ s=i d_{E}$ e logo $\left(E^{\prime \prime}, \psi\right)$ cinde. Recíprocamente, seja $\left(E^{\prime}, \varphi^{\prime}\right)$ uma extensão central de $G$ por $A$ e seja $E^{\prime \prime}=\{(x, y)$ : $\left.\varphi(x)=\varphi^{\prime}(y)\right\} \subset E \times E^{\prime}$. Considere a projecão $\pi_{1}: E^{\prime \prime} \rightarrow E,(x, y) \mapsto x$. Note que $\left(E^{\prime \prime}, \pi_{1}\right)$ é uma extensão central de $E$, $\operatorname{logo}$ existe um homomorfismo $s: E \rightarrow E^{\prime \prime}$ tal que $\pi_{1} \circ s=i d_{E}$. Considere agora a projecão $\pi_{2}: E^{\prime \prime} \rightarrow E^{\prime},(x, y) \mapsto y$ e defina $h: E \rightarrow E^{\prime}$ como sendo $h=\pi_{2} \circ s$. Se $x \in E$ e $s(x)=\left(x^{\prime}, y^{\prime}\right)$, então $\varphi\left(x^{\prime}\right)=\varphi^{\prime}\left(y^{\prime}\right)$ e $x=\pi_{1}(s(x))=\pi_{1}\left(x^{\prime}, y^{\prime}\right)=x^{\prime}$. Portanto

$$
\left(\varphi^{\prime} \circ h\right)(x)=\varphi^{\prime}\left(\pi_{2}(s(x))\right)=\varphi^{\prime}\left(\pi_{2}\left(x^{\prime}, y^{\prime}\right)\right)=\varphi^{\prime}\left(y^{\prime}\right)=\varphi\left(x^{\prime}\right)=\varphi(x) .
$$

Pelo Lema (2.2) e dado que $E$ é perfeito, temos que $(E, \varphi)$ é universal.

Proposição 2.3. Um grupo $G$ admite uma extensão central universal se, e somente se, $G$ é perfeito.

Demonstração. Seja $(E, \varphi)$ uma extensão central universal de $G$ por algum grupo abeliano $A$. Pela Proposição (2.2), $E$ é perfeito e dado que $\varphi(E)=G$, então

$$
[G, G]=[\varphi(E), \varphi(E)]=\varphi([E, E])=\varphi(E)=G
$$


Logo, $G$ é perfeito. Recíprocamente, suponha que $G$ é perfeito e escolha um grupo livre $F$ e um homomorfismo sobrejetor $\phi: F \rightarrow G$ e seja $R=\operatorname{Ker} \phi$. Note que $[R, F]$ é normal em $F$. Logo temos um homomorfismo sobrejetor $\varphi: F /[R, F] \rightarrow G \cong F / R$. Claramente, $(F /[R, F], \varphi)$ é uma extensão central de $G$ por $\operatorname{Ker} \varphi$. Note que $[F /[R, F], F /[R, F]]=[F, F] /[R, F]$ e pelo Lema (2.4), $\left([F, F] /[R, F],\left.\varphi\right|_{[F, F] /[R, F]}\right)$ é uma extensão central de $G$ por $\operatorname{Ker} \varphi$, onde $[F, F] /[R, F]$ é perfeito. Seja $(X, \psi)$ uma extensão central de $G$ por algum grupo abeliano $A^{\prime}$. Dado que $F$ é um grupo livre, existe um homomorfismo $h: F \rightarrow X$ tal que $\psi \circ h=\phi$. Dado que $(X, \psi)$ é uma extensão central, então $h([R, F])=1$. Logo temos um homomorfismo $h^{\prime}: F /[R, F] \rightarrow X$ tal que $\psi \circ h^{\prime}=\varphi$. Restringindo $\left.h^{\prime}\right|_{[F, F] /[R, F]}$ obtemos um homomorfismo $[F, F] /[R, F] \rightarrow G$ que satisfaz as condições do Lema (2.2) e é único. Portanto a extensão $\left([F, F] /[R, F],\left.\varphi\right|_{[F, F] /[R, F]}\right)$ é universal.

Na demonstração anterior, obtemos a sequência exata

$$
\left.0 \longrightarrow \operatorname{Ker} \varphi\right|_{[F, F] /[R, F]} \longrightarrow[F, F] /[R, F] \stackrel{\varphi}{\longrightarrow} G \rightarrow 1 .
$$

Note que $\left.\operatorname{Ker} \varphi\right|_{[F, F] /[R, F]}=(R \cap[F, F]) /[R, F]$. Note também que $(R /[R, R])_{G}=R /[R, F]$. Agora aplicando o Teorema (1.15), sobre a sequência exata

$$
1 \longrightarrow R \longrightarrow F \stackrel{\phi}{\longrightarrow} G \longrightarrow 1
$$

obtemos a sequência exata

$$
H_{2}(F) \longrightarrow H_{2}(G) \longrightarrow H_{1}(R)_{G} \longrightarrow H_{1}(F) \longrightarrow H_{1}(G) \longrightarrow 0 .
$$

Já que $G$ é perfeito, por (1.2) temos $H_{1}(G)=G /[G, G]=0$. Dado que $F$ é livre, por (1.4) temos que $H_{2}(F)=0$. Assim temos a sequência exata

$$
0 \longrightarrow H_{2}(G) \longrightarrow R /[R, F] \longrightarrow F /[F, F] \longrightarrow 0 \text {. }
$$

Portanto

$$
H_{2}(G) \cong \operatorname{Ker}(R /[R, F] \rightarrow F /[F, F])=(R \cap[F, F]) /[R, F]
$$

Assim obtemos a sequência exata que define a extensão central universal

$$
0 \longrightarrow H_{2}(G) \longrightarrow[F, F] /[R, F] \stackrel{\varphi}{\longrightarrow} G \longrightarrow 1 \text {. }
$$

LEMA 2.5. Seja $G$ um grupo perfeito. Então $H_{2}(G)=0$ se, e somente se, toda extensão central de $G$ cinde.

Demonstração. Dado que $G$ é perfeito, então temos a extensão central universal

$$
0 \longrightarrow H_{2}(G) \longrightarrow[F, F] /[R, F] \stackrel{\varphi}{\longrightarrow} G \longrightarrow 1,
$$

onde $1 \rightarrow R \rightarrow F \rightarrow G \rightarrow 1$ é uma presentação de $G$. Se $H_{2}(G)=0$, então a extensão central universal está dada pela sequência exata $1 \rightarrow 1 \rightarrow G \stackrel{i d_{G}}{\longrightarrow} G \rightarrow 1$. Seja $(E, \varphi)$ uma extensão 
central de $G$. Então existe um homomorfismo $h: G \rightarrow E$ tal que $\varphi \circ h=i d_{G}$, $\log$ o $(E, \varphi)$ cinde. Recíprocamente, suponha que toda extensão central de $G$ cinde. Seja $(E, \varphi)$ uma extensão central de $G$. Então existe um homomorfismo $s: G \rightarrow E$ tal que $\varphi \circ s=i d_{G}$. Logo a extensão central de $G$ dada por

$$
1 \rightarrow 1 \rightarrow G \stackrel{i d_{G}}{\longrightarrow} G \rightarrow 1
$$

é universal. Portanto $H_{2}(G)=0$.

COROLÁRIO 2.1. Seja $G$ um grupo perfeito. Então uma extensão central $(E, \varphi)$ de G é universal se, e somente se, $H_{1}(E)=H_{2}(E)=0$.

Demonstração. Seja $(E, \varphi)$ uma extensão central de $G$. Pela Proposição (2.2), $(E, \varphi)$ é universal se, e somente se, $E$ é perfeito e toda extensão central de $E$ cinde. Note que $E$ é perfeito se, e somente se, $H_{1}(E)=0$ e pelo Lema (2.5), $H_{2}(E)=0$ se, e somente se toda extensão central de $E$ cinde. $\mathrm{O}$ resultado segue de forma direita.

\section{$2.3 \quad K_{2}(R)$}

Com estas ferramentas já definidas, começaremos o estudo do $K_{2}-$ grupo do anel $R$. Para $n \geq 3$, defina o $n$-ésimo grupo Steinberg do anel $R$, denotado $S t_{n}(R)$, como sendo o grupo livre gerado pelos símbolos $x_{i j}^{n}(\lambda)$ com $1 \leq i, j \leq n, i \neq j$ e $\lambda \in R$, satisfazendo as relações de Steinberg

- $\quad x_{i j}^{n}(\lambda) x_{i j}^{n}(\tau)=x_{i j}^{n}(\lambda+\tau)$, se $i \neq j$

$$
\text { - }\left[x_{i j}^{n}(\lambda), x_{k l}^{n}(\tau)\right]= \begin{cases}1 & \text { se } j \neq k \text { e } i \neq l \\ x_{i l}^{n}(\lambda \tau) & \text { se } j=k \text { e } i \neq l \\ x_{k j}^{n}(-\tau \lambda) & \text { se } j \neq k \text { e } i=l\end{cases}
$$

Dado que as matrizes elementares $e_{i, j}^{n}(\lambda) \in E_{n}(R)$ satisfazem as relações anteriores, então existe um homomorfismo sobrejetor $\phi_{n}: S t_{n}(R) \rightarrow E_{n}(R)$ definido por $x_{i j}^{n}(\lambda) \mapsto e_{i, j}^{n}(\lambda)$. As relações de Steinberg para $n+1$ contêm as relações de Steinberg para $n$, assim existe um homomorfismo $S t_{n}(R) \rightarrow S t_{n+1}(R)$ definido por $x_{i j}^{n}(\lambda) \mapsto x_{i j}^{n+1}(\lambda)$. Definimos o grupo Steinberg do anel $R$ como sendo

$$
S t(R):=\underset{\longrightarrow}{\lim S t_{n}}(R),
$$

induzindo assim um morfismo sobrejetor $\phi: S t(R) \rightarrow E(R)$. Note que pelas relações de Steinberg, $S t(R)$ é um grupo perfeito.

DEFINIÇão 2.3. Seja $R$ um anel com unidade. Definimos o $K_{2}-$ grupo de $R$, denotado por $K_{2}(R)$, como sendo

$$
K_{2}(R):=\operatorname{Ker}(\phi: S t(R) \rightarrow E(R))
$$


Temos assim a sequência exata

$$
1 \longrightarrow K_{2}(R) \longrightarrow S t(R) \stackrel{\phi}{\longrightarrow} E(R) \longrightarrow 1 \text {. }
$$

Agora se $\alpha: R \rightarrow S$ é um homomorfismo de anéis, então temos um homomorfismo de grupos

$$
\alpha^{\prime}: S t(R) \longrightarrow S t(S), \quad x_{i j}^{n}(\lambda) \longmapsto x_{i j}^{n}(\alpha(\lambda))
$$

É claro que $\alpha^{\prime}\left(K_{2}(R)\right) \subseteq K_{2}(S)$. Então restringindo o homomorfismo anterior a $K_{2}(R)$ obtemos um homomorfismo de grupos bem definido $K_{2}(\alpha): K_{2}(R) \rightarrow K_{2}(S)$. É fácil verificar que $K_{2}(-)$ é um funtor da categoria de anéis na categoria de grupos.

Proposição 2.4. Seja $R$ um anel. Então $K_{2}(R)=Z(S t(R))$.

Demonstração. Se $A \in Z(E(R))$, então existe $n>0$ tal que $A \in E_{n}(R)$. Assim, em $E_{2 n}(R)$ temos

$$
\left(\begin{array}{cc}
A & A \\
0 & I_{n}
\end{array}\right)=\left(\begin{array}{cc}
A & 0 \\
0 & I_{n}
\end{array}\right)\left(\begin{array}{cc}
I_{n} & I_{n} \\
0 & I_{n}
\end{array}\right)=\left(\begin{array}{cc}
I_{n} & I_{n} \\
0 & I_{n}
\end{array}\right)\left(\begin{array}{cc}
A & 0 \\
0 & I_{n}
\end{array}\right)=\left(\begin{array}{cc}
A & I_{n} \\
0 & I_{n}
\end{array}\right)
$$

Logo, $A=I$ e portanto $Z(E(R))=1$. Se $B \in Z(S t(R))$, então $\phi(B) \in Z(E(R))=1$. Assim, temos que $B \in K_{2}(R)$. Por outro lado, fixe $m>0$ e defina os subgrupos abelianos de $S t(R)$,

$$
\begin{aligned}
& A_{m}:=\left\langle x_{i m}^{n}(\lambda): i \neq m, \lambda \in R, n>0\right\rangle, \\
& B_{m}:=\left\langle x_{m j}^{n}(\lambda): j \neq m, \lambda \in R, n>0\right\rangle .
\end{aligned}
$$

Se $p \neq q$ e $n>0$, então

$$
\begin{aligned}
x_{p q}^{n}(\lambda) \in A_{m} & \text { se } q=m, \\
x_{p q}^{n}(\lambda) \in B_{m} & \text { se } p=m, \\
x_{p q}^{n}(\lambda)=\left[x_{p m}^{n}(\lambda), x_{m q}^{n}(1)\right] \in\left[A_{m}, B_{m}\right] & \text { se } p \neq m, q \neq m .
\end{aligned}
$$

Note que $\phi$ restringida a $A_{m}$ e $B_{m}$ é injetora. Agora pelas relações de Steinberg, temos que

- se $p \neq m$ e $n>0$, então

$$
x_{p q}^{n}(\lambda) x_{i m}^{n}(\tau)\left(x_{p q}^{n}(\lambda)\right)^{-1}= \begin{cases}x_{i m}^{n}(\tau) & \text { se } q \neq i \\ x_{p m}^{n}(\lambda \tau) x_{i m}^{n}(\tau) & \text { se } q=i\end{cases}
$$

- se $q \neq m$ e $n>0$, então

$$
x_{p q}^{n}(\lambda) x_{m j}^{n}(\tau)\left(x_{p q}^{n}(\lambda)\right)^{-1}= \begin{cases}x_{m j}^{n}(\tau) & \text { se } p \neq j \\ x_{m q}^{n}(-\tau \lambda) x_{m j}^{n}(\tau) & \text { se } p=j .\end{cases}
$$


Portanto para $p \neq m, q \neq m$ e $n>0$, obtemos

$$
\begin{array}{ll}
x_{p q}^{n}(\lambda) A_{m}\left(x_{p q}^{n}(\lambda)\right)^{-1} & \subseteq A_{m}, \\
x_{p q}^{n}(\lambda) B_{m}\left(x_{p q}^{n}(\lambda)\right)^{-1} & \subseteq B_{m} .
\end{array}
$$

Agora, seja $x \in K_{2}(R)$ e seja

$$
x=\prod_{l=1}^{s} x_{i_{l} j_{l}}^{n}\left(\lambda_{l}\right), \quad \text { onde } \quad \prod_{l=1}^{s} e_{i_{l}, j_{l}}^{n}\left(\lambda_{l}\right)=1 \quad \text { em } E(R) .
$$

Se $N>i_{l}, j_{l}$, para todo $1 \leq l \leq s$, então para todo $\alpha \in A_{N}$ e $\beta \in B_{N}$ temos que $x \alpha x^{-1} \in A_{N}$ e $x \beta x^{-1} \in B_{N}$. Logo

$$
\phi\left(x \alpha x^{-1}\right)=\phi(\alpha), \quad \phi\left(x \beta x^{-1}\right)=\phi(\beta) .
$$

Dado que $\left.\phi\right|_{A_{N}}$ e $\left.\varphi\right|_{B_{N}}$ são injetoras, $x \in Z\left(A_{N}\right)$ e $x \in Z\left(B_{N}\right)$. Portanto $x \in Z(S t(R))$.

Assim, $K_{2}(R)$ é um grupo abeliano. É fácil ver que $K_{2}(-)$ é um funtor da categoria de anéis na categoria de grupos abelianos.

Proposição 2.5. Seja $R$ um anel com unidade. Então $(S t(R), \phi)$ é uma extensão central universal de $E(R)$ por $K_{2}(R)$.

Demonstração. Pela Proposição (2.2), é suficiente provar que toda extensão central de $S t(R)$ cinde. Seja $(E, \psi)$ uma extensão central de $S t(R)$. Definimos o homomorfismo

$$
s: S t(R) \longrightarrow E, \quad x_{i j}^{n}(\lambda) \longmapsto\left[\psi^{-1}\left(x_{i k}^{n}(1)\right), \psi^{-1}\left(x_{k j}^{n}(\lambda)\right)\right]
$$

para algum $k \neq i, j$. Notemos que se $i, j, k, l$ são distintos e $\lambda, \tau \in R$, então

$$
\left[\psi^{-1}\left(x_{i k}^{n}(\lambda)\right), \psi^{-1}\left(x_{k j}^{n}(\tau)\right)\right]=\left[\psi^{-1}\left(x_{i l}^{n}(\lambda)\right), \psi^{-1}\left(x_{l j}^{n}(\tau)\right)\right] .
$$

Logo $s$ é independente da escolha de $k$. Note que se $x, y \in \operatorname{St}(R)$ e $p, p^{\prime}, q, q^{\prime} \in E$ tais que $\psi(p)=$ $\psi\left(p^{\prime}\right)=x$ e $\psi(q)=\psi\left(q^{\prime}\right)=y$, então $[p, q]=\left[p^{\prime}, q^{\prime}\right]$. Logo tem sentido definir $\left[\psi^{-1}(x), \psi^{-1}(y)\right]$. Note que $s\left(x_{i j}^{n}(\lambda)\right)$ satisfaz as relacões de Steinberg e $s\left(x_{i j}^{n}(\lambda)\right) \in \psi^{-1}\left(x_{i j}^{n}(\lambda)\right)$. Portanto $(\psi \circ s)=i d_{S t(R)}$ e portanto $(E, \psi)$ cinde.

Temos assim a sequência exata que define a extensão central universal de $E(R)$ por $K_{2}(R)$

$$
1 \longrightarrow K_{2}(R) \longrightarrow S t(R) \stackrel{\phi}{\longrightarrow} E(R) \longrightarrow 1
$$

Pela Proposição (2.1) temos que $E(R)$ é perfeito e pelo Corolário (2.1) temos que

$$
H_{1}(S t(R))=0, \quad H_{2}(S t(R))=0 .
$$

TEOREMA 2.1. Seja $R$ um anel com unidade. Então $K_{2}(R) \cong H_{2}(E(R))$. 
Demonstração. A sequência exata

$$
1 \longrightarrow K_{2}(R) \longrightarrow S t(R) \stackrel{\phi}{\longrightarrow} E(R) \longrightarrow 1
$$

induz a sequência espectral de Lyndon/Hochschild-Serre

$$
E_{p, q}^{2}=H_{p}\left(E(R), H_{q}\left(K_{2}(R)\right)\right) \Longrightarrow H_{p+q}(S t(R)) .
$$

Como $K_{2}(R)$ é o centro de $S t(R), E(R)$ age trivialmente sobre $H_{q}\left(K_{2}(R)\right)$. Calculamos alguns elementos da sequência espectral:

- Se $q=0$, então

$$
E_{p, 0}^{2}=H_{p}\left(E(R), H_{0}\left(K_{2}(R)\right)\right)=H_{p}(E(R))
$$

- Se $p=1$, então pelo Exemplo 1.14 e do fato que $E(R)$ é perfeito, temos

$$
E_{1, q}^{2}=H_{1}\left(E(R), H_{q}\left(K_{2}(R)\right)\right)=H_{1}(E(R)) \otimes H_{q}\left(K_{2}(R)\right)=0 .
$$

- Se $p=0$ e $q=1$, então

$$
E_{0,1}^{2}=H_{0}\left(E(R), H_{1}\left(K_{2}(R)\right)\right)=H_{0}\left(E(R), K_{2}(R)\right)=K_{2}(R) .
$$

Então a segunda folha desta sequência espectral é dada por

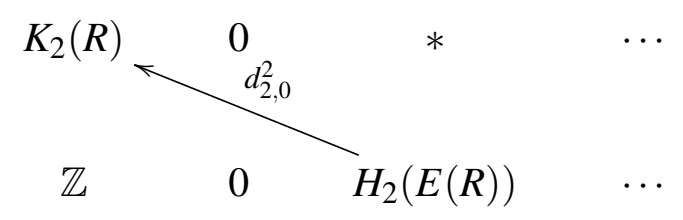

$\operatorname{Assim} E_{0,1}^{\infty}=E_{0,1}^{3}=\operatorname{Coker} d_{2,0}^{2}$ e $E_{2,0}^{\infty} \cong E_{2,0}^{3}=\operatorname{Ker} d_{2,0}^{2}$. Considere as filtrações

$$
\begin{gathered}
0=F_{-1} H_{1}(S t(R)) \subseteq F_{0} H_{1}(S t(R)) \subseteq F_{1} H_{1}(S t(R))=H_{1}(S t(R)) \\
0=F_{-1} H_{2}(\operatorname{St}(R)) \subseteq F_{0} H_{2}(\operatorname{St}(R)) \subseteq F_{1} H_{2}(\operatorname{St}(R)) \subseteq F_{2} H_{2}(\operatorname{St}(R))=H_{2}(\operatorname{St}(R)),
\end{gathered}
$$

induzidas pela sequência espectral $E_{p, q}^{2}$. Como $H_{1}(S t(R))=H_{2}(\operatorname{St}(R))=0, E_{0,1}^{\infty}=E_{2,0}^{\infty}=0$. Assim $E_{0,1}^{3}=E_{2,0}^{3}=0$ e $\operatorname{logo} d_{2,0}^{2}: H_{2}(E(R)) \rightarrow K_{2}(R)$ é um isomorfismo. Portanto $K_{2}(R) \cong$ $\mathrm{H}_{2}(\mathrm{E}(\mathrm{R}))$.

\subsection{Sequência Espectral Principal}

Seja $R$ um anel local com ideal maximal $\mathfrak{m}$. Para $n \geq 0$ seja

$$
X_{n}:=\left\{\left(\left\langle v_{0}\right\rangle, \ldots,\left\langle v_{n}\right\rangle\right): v_{i} \in R^{2}, 0 \neq \overline{v_{i}} \in R / \mathfrak{m} \text { e }\left\langle\overline{v_{i}}\right\rangle \neq\left\langle\overline{v_{j}}\right\rangle, \text { se } i \neq j\right\}
$$


onde $\left\langle v_{i}\right\rangle:=R v_{i}$. Definimos para $n \geq 0$ os $\mathbb{Z}$-módulos livres $C_{n}\left(R^{2}\right):=\mathbb{Z}\left(X_{n}\right)$ e defina os homomorfismos

$$
\partial_{0}: C_{0}\left(R^{2}\right) \longrightarrow \mathbb{Z}, \quad \sum_{j} m_{j}\left(\left\langle v_{0}^{j}\right\rangle\right) \longmapsto \sum_{j} m_{j}
$$

e para $n \geq 1$,

$$
\begin{aligned}
\partial_{n}: C_{n}\left(R^{2}\right) & \longrightarrow C_{n-1}\left(R^{2}\right) \\
\sum_{j} m_{j}\left(\left\langle v_{0}^{j}\right\rangle, \ldots,\left\langle v_{n}^{j}\right\rangle\right) & \longmapsto \sum_{j} m_{j}\left(\sum_{k=0}^{n}(-1)^{k}\left(\left\langle v_{0}^{j}\right\rangle, \ldots, \widehat{\left\langle v_{k}^{j}\right\rangle}, \ldots,\left\langle v_{n}^{j}\right\rangle\right)\right),
\end{aligned}
$$

onde

$$
\left(\left\langle v_{0}^{j}\right\rangle, \ldots, \widehat{\left\langle v_{k}^{j}\right\rangle}, \ldots,\left\langle v_{n}^{j}\right\rangle\right)=\left(\left\langle v_{0}^{j}\right\rangle, \ldots,\left\langle v_{k-1}^{j}\right\rangle,\left\langle v_{k+1}^{j}\right\rangle, \ldots,\left\langle v_{n}^{j}\right\rangle\right) .
$$

LEMA 2.6. Seja $R$ um anel local com ideal maximal $\mathfrak{m}$. Então a sequência $C_{\bullet}\left(R^{2}\right) \rightarrow \mathbb{Z}: \quad \cdots \rightarrow C_{n+1}\left(R^{2}\right) \stackrel{\partial_{n+1}}{\longrightarrow} C_{n}\left(R^{2}\right) \stackrel{\partial_{n}}{\longrightarrow} C_{n-1}\left(R^{2}\right) \rightarrow \cdots \rightarrow C_{0}\left(R^{2}\right) \stackrel{\partial_{0}}{\longrightarrow} \mathbb{Z} \rightarrow 0$, é um complexo de cadeias. Além disso, se $R / \mathfrak{m}$ for infinito, então $C_{\bullet}\left(R^{2}\right) \rightarrow \mathbb{Z}$ é exato.

Demonstração. Dado que $\partial_{0}\left(\left\langle v_{0}^{j}\right\rangle\right)=1$, então $\partial_{0}$ é sobrejetor. Além disso

$$
\partial_{0} \circ \partial_{1}\left(\left\langle v_{0}\right\rangle,\left\langle v_{1}\right\rangle\right)=\partial_{0}\left(\left\langle v_{1}\right\rangle-\left\langle v_{0}\right\rangle\right)=1-1=0 .
$$

Assim, Im $\partial_{1} \subset \operatorname{Ker} \partial_{0}$. Agora para $n \geq 1$, temos

$$
\begin{aligned}
\partial_{n} \circ \partial_{n+1}\left(\left\langle v_{0}\right\rangle, \ldots,\left\langle v_{n+1}\right\rangle\right) & =\partial_{n}\left(\sum_{j=0}^{n+1}(-1)^{j}\left(\left\langle v_{0}\right\rangle, \ldots, \widehat{\left\langle v_{j}\right\rangle}, \ldots,\left\langle v_{n+1}\right\rangle\right)\right) \\
& =\sum_{j=0}^{n+1}(-1)^{j} \partial_{n}\left(\left\langle v_{0}\right\rangle, \ldots, \widehat{\left\langle v_{j}\right\rangle}, \ldots,\left\langle v_{n+1}\right\rangle\right) \\
& =(-1)^{j+k} \sum_{j=1}^{n+1} \sum_{k=0}^{j-1}\left(\left\langle v_{0}\right\rangle, \ldots, \widehat{\left\langle v_{k}\right\rangle}, \ldots, \widehat{\left\langle v_{j}\right\rangle}, \ldots,\left\langle v_{n+1}\right\rangle\right) \\
& -(-1)^{j+k} \sum_{k=1}^{n+1} \sum_{j=0}^{k-1}\left(\left\langle v_{0}\right\rangle, \ldots, \widehat{\left\langle v_{j}\right\rangle}, \ldots, \widehat{\left\langle v_{k}\right\rangle}, \ldots,\left\langle v_{n+1}\right\rangle\right) \\
& =(-1)^{j+k} \sum_{j=1}^{n+1} \sum_{k=0}^{j-1}\left(\left\langle v_{0}\right\rangle, \ldots, \widehat{\left\langle v_{k}\right\rangle}, \ldots, \widehat{\left\langle v_{j}\right\rangle}, \ldots,\left\langle v_{n+1}\right\rangle\right) \\
& -(-1)^{j+k} \sum_{k=1}^{n+1} \sum_{j=0}^{k-1}\left(\left\langle v_{0}\right\rangle, \ldots, \widehat{\left\langle v_{j}\right\rangle}, \ldots, \widehat{\left\langle v_{k}\right\rangle}, \ldots,\left\langle v_{n+1}\right\rangle\right) \\
& =0 .
\end{aligned}
$$

Assim para $n \geq 1$, Im $\partial_{n+1} \subset \operatorname{Ker} \partial_{n}$. Portanto $C_{\bullet}\left(R^{2}\right) \rightarrow \mathbb{Z}$ é um complexo de cadeias. Seja $R / \mathfrak{m}$ um corpo infinito e seja $x=\sum_{j} m_{j}\left(\left\langle v_{0}^{j}\right\rangle, \ldots,\left\langle v_{n}^{j}\right\rangle\right) \in C_{n}\left(R^{2}\right)$ tal que $\partial_{n}(x)=0$, isto é,

$$
\sum_{j} m_{j}\left(\sum_{k=0}^{n}(-1)^{k}\left(\left\langle v_{0}^{j}\right\rangle, \ldots, \widehat{\left\langle v_{k}^{j}\right\rangle}, \ldots,\left\langle v_{n}^{j}\right\rangle\right)\right)=0 .
$$


Como $R / \mathfrak{m}$ é infinito, existe $w \in R^{2}$ tal que $\langle\bar{w}\rangle \neq\left\langle\bar{v}_{i}^{j}\right\rangle$, para todo $i=0, \ldots, n$ e para todo $j$. Se

$$
y=(-1)^{n+1} \sum_{j} m_{j}\left(\left\langle v_{0}^{j}\right\rangle, \ldots,\left\langle v_{n}^{j}\right\rangle,\langle w\rangle\right) \in C_{n+1}\left(R^{2}\right),
$$

então

$$
\begin{aligned}
\partial_{n+1}(y) & =(-1)^{n+1} \sum_{j} m_{j}\left(\sum_{k=0}^{n}(-1)^{k}\left(\left\langle v_{0}^{j}\right\rangle, \ldots, \widehat{\left\langle v_{k}^{j}\right\rangle}, \ldots,\left\langle v_{n}^{j}\right\rangle,\langle w\rangle\right)\right) \\
& +(-1)^{n+1} \sum_{j} m_{j}(-1)^{n+1}\left(\left\langle v_{0}^{j}\right\rangle, \ldots,\left\langle v_{n}^{j}\right\rangle\right) \\
& =(-1)^{n+1}(-1)^{n+1} \sum_{j} m_{j}\left(\left\langle v_{0}^{j}\right\rangle, \ldots,\left\langle v_{n}^{j}\right\rangle\right) \\
& =x \in \operatorname{Im} \partial_{n+1} .
\end{aligned}
$$

Portanto, $C_{\bullet}\left(R^{2}\right) \rightarrow \mathbb{Z}$ é um complexo exato.

Seja $G$ um grupo agindo sobre o conjunto $X$. Definimos a órbita e o subgrupo estabilizador de $x \in X$ como sendo

$$
\begin{aligned}
o(x) & :=\{g \cdot x: g \in G\} \subseteq X, \\
G_{x} & :=\{g: g \cdot x=x\} \subseteq G .
\end{aligned}
$$

Dizemos que um subconjunto $T$ de $X$ é um conjunto de representantes das órbitas de $X$ se $T$ corta cada órbita da ação de $G$ em um único ponto. Assim podemos escrever $X$ como uma reunião disjunta das órbitas dos elementos de $T$, isto é

$$
X=\bigsqcup_{x \in T} o(x) .
$$

LEMA 2.7. Seja $G$ um grupo agindo sobre o conjunto $X$ e seja $T$ um conjunto de representantes das órbitas de X. Então

$$
\mathbb{Z} X \cong \bigoplus_{x \in T}\left(\mathbb{Z} G \otimes_{G_{x}} \mathbb{Z}\right)
$$

Demonstração. Considere o $G$-homomorfismo

$$
\begin{aligned}
\phi: \bigoplus_{x \in T}\left(\mathbb{Z} G \otimes_{G_{x}} \mathbb{Z}\right) & \longrightarrow \mathbb{Z} X \\
\left(\sum_{g_{x} \in G} n_{g_{x}} g_{x} \otimes m_{x}\right)_{x \in T} & \longmapsto \sum_{x \in T}\left(\sum_{g_{x} \in G}\left(m_{x} n_{g_{x}}\right) \cdot\left(g_{x} x\right)\right) .
\end{aligned}
$$

Seja $y^{\prime}=\sum_{y \in X} n_{y} y \in \mathbb{Z} X$. Então para todo $y \in X$, existe $g_{y} \in G$ e um único $x_{y} \in T$ tal que $g_{y} x_{y}=y$. Definimos a aplicação $\phi$ como sendo

$$
\begin{aligned}
\psi: \mathbb{Z} X & \longrightarrow \bigoplus_{x \in T}\left(\mathbb{Z} G \otimes_{G_{x}} \mathbb{Z}\right), \\
y^{\prime} & \longmapsto\left(g_{y} \otimes n_{y}\right)_{x \in T} .
\end{aligned}
$$

Suponha que existem $g_{y}, g_{y}^{\prime} \in G$ tais que $g_{y} x_{y}=y=g_{y}^{\prime} x_{y}$. Então $g_{y}^{-1} g_{y}^{\prime} \in G_{x_{y}}$ e portanto

$$
g_{y}^{\prime} \otimes n_{y}=\left(g_{y} g_{y}^{-1}\right) g_{y}^{\prime} \otimes n_{y}=g_{y}\left(g_{y}^{-1} g_{y}^{\prime}\right) \otimes n_{y}=g_{y} \otimes g_{y}^{-1} g_{y}^{\prime} \cdot n_{y}=g_{y} \otimes n_{y} .
$$

Logo a aplicação anterior é um $G$-homomorfismo bem definido e é a inversa de $\phi$. 
EXEMPLO 2.4. Definimos para todo $n \geq 0$, uma ação do grupo $G L_{2}(R)$ sobre $X_{n}$ como segue

$$
A \cdot\left(\left\langle v_{0}\right\rangle, \ldots,\left\langle v_{n}\right\rangle\right):=\left(\left\langle A v_{0}\right\rangle, \ldots,\left\langle A v_{n}\right\rangle\right),
$$

onde $A \in G L_{2}(R)$ e $\left(\left\langle v_{0}\right\rangle, \ldots,\left\langle v_{n}\right\rangle\right) \in X_{n}$. Assim

$$
C_{n}\left(R^{2}\right)=\mathbb{Z} X_{n} \cong \bigoplus_{x \in T_{n}}\left(\mathbb{Z} G L_{2}(R) \otimes_{\left(G L_{2}(R)\right)_{x}} \mathbb{Z}\right)
$$

onde $T_{n}$ é um conjunto de representantes das órbitas da ação de $G L_{2}(R)$ sobre $X_{n}$. Portanto, se $R / \mathfrak{m}$ for um corpo infinito, então $C_{\bullet}\left(R^{2}\right) \rightarrow \mathbb{Z}$ é um complexo exato de $G L_{2}(R)$-módulos.

Proposição 2.6. Seja $R$ um anel local com ideal maximal $\mathfrak{m}$. Então existe uma sequência espectral do primeiro quadrante

$$
E_{p, q}^{1}=H_{q}\left(G L_{2}(R), C_{p}\left(R^{2}\right)\right) \Longrightarrow H_{p+q}\left(G L_{2}(R), C_{\bullet}\left(R^{2}\right)\right)
$$

$S e R / \mathfrak{m}$ for infinito, então

$$
E_{p, q}^{1}=H_{q}\left(G L_{2}(R), C_{p}\left(R^{2}\right)\right) \Longrightarrow H_{p+q}\left(G L_{2}(R)\right)
$$

Demonstração. Segue-se do Teorema (1.14), aplicado para o complexo $C_{\bullet}\left(R^{2}\right) \rightarrow \mathbb{Z}$.

\subsection{O teorema de Van der Kallen-Matsumoto}

Seja $R$ um anel local com ideal maximal $\mathfrak{m}$ tal que $R / \mathfrak{m}$ é um corpo infinito. Primero vamos demonstrar que o grupo $G L_{2}(R)$ age transitivamente sobre $X_{n}$ para $n=0,1,2$.

Seja $n=0$ e seja $v=\left(\begin{array}{l}v_{1} \\ v_{2}\end{array}\right) \in R^{2}$ tal que $\bar{v} \neq 0$. Sem perda de generalidade podemos supor $\overline{v_{1}} \neq 0$. Então é suficiente provar que existe $A_{0} \in G L_{2}(R)$ tal que $A_{0} \cdot\left(\left\langle e_{1}\right\rangle\right)=(\langle v\rangle)$, onde $e_{1}=\left(\begin{array}{l}1 \\ 0\end{array}\right)$. Se $A_{0}=\left(\begin{array}{ll}v_{1} & 0 \\ v_{2} & 1\end{array}\right) \in G L_{2}(R)$, então

$$
A_{0} \cdot\left(\left\langle e_{1}\right\rangle\right)=\left(\left\langle A_{0} e_{1}\right\rangle\right)=(\langle v\rangle)
$$

Seja $n=1$ e seja $(\langle v\rangle,\langle w\rangle) \in X_{1}$, onde $v=\left(\begin{array}{l}v_{1} \\ v_{2}\end{array}\right)$ e $w=\left(\begin{array}{l}w_{1} \\ w_{2}\end{array}\right)$. Então é suficiente provar que existe $A_{1} \in G L_{2}(R)$ tal que

$$
A_{1} \cdot\left(\left\langle e_{1}\right\rangle,\left\langle e_{2}\right\rangle\right)=(\langle v\rangle,\langle w\rangle)
$$

onde $e_{2}=\left(\begin{array}{l}0 \\ 1\end{array}\right)$. Considere $A_{1}=\left(\begin{array}{ll}v_{1} & w_{1} \\ v_{2} & w_{2}\end{array}\right)$. Como $\langle\bar{v}\rangle \neq\langle\bar{w}\rangle$, então $A_{1} \in G L_{2}(R)$ e temos que

$$
A_{1} \cdot\left(\left\langle e_{1}\right\rangle,\left\langle e_{2}\right\rangle\right)=\left(\left\langle A_{1} e_{1}\right\rangle,\left\langle A_{1} e_{2}\right\rangle\right)=(\langle v\rangle,\langle w\rangle)
$$


Se $\left(\left\langle v_{0}\right\rangle,\left\langle v_{1}\right\rangle,\left\langle v_{2}\right\rangle\right) \in X_{2}$, então é suficiente provar que existe $A_{2} \in G L_{2}(R)$ tal que

$$
A_{2} \cdot\left(\left\langle v_{0}\right\rangle,\left\langle v_{1}\right\rangle,\left\langle v_{2}\right\rangle\right)=\left(\left\langle e_{1}\right\rangle,\left\langle e_{2}\right\rangle,\left\langle e_{1}+e_{2}\right\rangle\right) .
$$

Dado que $\left\{v_{0}, v_{1}\right\}$ é uma base para $R^{2}$, existe $a \in R^{\times}$tal que $v_{2}=v_{0}+a v_{1}$. Se $A_{2}=\left(\begin{array}{cc}v_{0}^{1} & a v_{1}^{1} \\ v_{0}^{2} & a v_{1}^{2}\end{array}\right) \in$ $G L_{2}(R)$, onde $v_{0}=\left(\begin{array}{l}v_{0}^{1} \\ v_{0}^{2}\end{array}\right), v_{1}=\left(\begin{array}{l}v_{1}^{1} \\ v_{1}^{2}\end{array}\right)$, então $A_{2} \cdot\left(\left\langle e_{1}\right\rangle,\left\langle e_{2}\right\rangle,\left\langle e_{1}+e_{2}\right\rangle\right)=\left(\left\langle v_{0}\right\rangle,\left\langle v_{1}\right\rangle,\left\langle v_{2}\right\rangle\right)$.

Para facilitar nossos cálculos, sejam

$$
x_{0}:=\left(\left\langle e_{1}\right\rangle\right) \in X_{0}, \quad x_{1}:=\left(\left\langle e_{1}\right\rangle,\left\langle e_{2}\right\rangle\right) \in X_{1}, \quad x_{2}:=\left(\left\langle e_{1}\right\rangle,\left\langle e_{2}\right\rangle,\left\langle e_{1}+e_{2}\right\rangle\right) \in X_{2} .
$$

Agora é fácil verificar que para o caso $n=3$ e $n=4$ temos

$$
X_{3}=\bigsqcup_{a \in A} o\left(x_{a}\right), \quad X_{4}=\bigsqcup_{(a, b) \in B} o\left(x_{a, b}\right),
$$

onde $x_{a}:=\left(\left\langle e_{1}\right\rangle,\left\langle e_{2}\right\rangle,\left\langle e_{1}+e_{2}\right\rangle,\left\langle e_{1}+a e_{2}\right\rangle\right), x_{a, b}:=\left(\left\langle e_{1}\right\rangle,\left\langle e_{2}\right\rangle,\left\langle e_{1}+e_{2}\right\rangle,\left\langle e_{1}+a e_{2}\right\rangle,\left\langle e_{1}+b e_{2}\right\rangle\right)$, $A:=\left\{a \in R: a, 1-a \in R^{\times}\right\}$e $B:=\left\{(a, b) \in R^{2}: a, 1-a, b, 1-b, a-b \in R^{\times}\right\}$.

Agora pelo Lema (2.7), temos

$$
\begin{aligned}
& C_{0}\left(R^{2}\right) \cong \mathbb{Z} G L_{2}(R) \otimes_{\left(G L_{2}(R)\right)_{x_{0}}} \mathbb{Z}, \\
& C_{1}\left(R^{2}\right) \cong \mathbb{Z} G L_{2}(R) \otimes_{\left(G L_{2}(R)\right)_{x_{1}}} \mathbb{Z}, \\
& C_{2}\left(R^{2}\right) \cong \mathbb{Z} G L_{2}(R) \otimes_{\left(G L_{2}(R)\right)_{x_{2}}} \mathbb{Z}, \\
& C_{3}\left(R^{2}\right) \cong \bigoplus_{a \in A}\left(\mathbb{Z} G L_{2}(R) \otimes_{\left(G L_{2}(R)\right)_{x_{a}}} \mathbb{Z}\right), \\
& C_{4}\left(R^{2}\right)=\bigoplus_{(a, b) \in B}\left(\mathbb{Z} G L_{2}(R) \otimes_{\left(G L_{2}(R)\right)_{x_{a, b}}} \mathbb{Z}\right) .
\end{aligned}
$$

Fazendo os cálculos, obtemos os seguintes subgrupos estabilizadores de $x_{0}, x_{1}, x_{2}, x_{a}, x_{a, b}$ :

$$
\begin{aligned}
\left(G L_{2}(R)\right)_{x_{0}} & =B_{2}:=\left\{\left(\begin{array}{ll}
a & b \\
0 & c
\end{array}\right): a, c \in R^{\times}, b \in R\right\}, \\
\left(G L_{2}(R)\right)_{x_{1}} & =T_{2}:=\left\{\left(\begin{array}{ll}
a & 0 \\
0 & b
\end{array}\right): a, b \in R^{\times}\right\} \cong R^{\times} \times R^{\times}, \\
\left(G L_{2}(R)\right)_{x_{2}} & =R^{\times} I_{2} \cong R^{\times}, \\
\left(G L_{2}(R)\right)_{x_{a}} & =R^{\times} I_{2} \cong R^{\times}, \\
\left(G L_{2}(R)\right)_{x_{a, b}} & =R^{\times} I_{2} \cong R^{\times} .
\end{aligned}
$$

Logo pelo Lema de Shapiro (1.4) e o Exemplo(1.7) temos

$$
\begin{aligned}
H_{q}\left(G L_{2}(R), C_{p}\left(R^{2}\right)\right) & =H_{q}\left(G L_{2}(R), \bigoplus_{x \in T_{p}}\left(\mathbb{Z} G L_{2}(R) \otimes_{\left(G L_{2}(R)\right)_{x}} \mathbb{Z}\right)\right) \\
& =\bigoplus_{x \in T_{p}} H_{q}\left(G L_{2}(R), \mathbb{Z} G L_{2}(R) \otimes_{\left(G L_{2}(R)\right)_{x}} \mathbb{Z}\right) \\
& =\bigoplus_{x \in T_{p}} H_{q}\left(\left(G L_{2}(R)\right)_{x}\right),
\end{aligned}
$$


onde $T_{p}$ é o conjunto de representantes das órbitas da ação de $G L_{2}(R)$ sobre $X_{p}$ e $\left(G L_{2}(R)\right)_{x}$ é o subgrupo estabilizador de $x \in T_{p}$. Então temos

$$
H_{q}\left(G L_{2}(R), C_{p}\left(R^{2}\right)\right)= \begin{cases}H_{q}\left(B_{2}\right), & p=0, \\ H_{q}\left(T_{2}\right), & p=1, \\ H_{q}\left(R^{\times}\right), & p=2, \\ \bigoplus_{a \in A} H_{q}\left(R^{\times}\right)[a], & p=3, \\ \bigoplus_{(a, b) \in B} H_{q}\left(R^{\times}\right)[a, b], & p=4,\end{cases}
$$

onde $B_{2}=\left(\begin{array}{cc}R^{\times} & R \\ 0 & R^{\times}\end{array}\right)$e $T_{2}=\left(\begin{array}{cc}R^{\times} & 0 \\ 0 & R^{\times}\end{array}\right) \cong R^{\times} \times R^{\times}$. Defina

$$
Q(R):=\bigoplus_{a \in A} \mathbb{Z}[a], \quad R(R):=\bigoplus_{(a, b) \in B} \mathbb{Z}[a, b] .
$$

Agora pela Proposição (1.4), $H_{i}\left(T_{2}\right) \cong H_{i}\left(B_{2}\right)$. Assim a primeira folha da sequência dada na Proposição (2.6) está dada por

$$
\begin{aligned}
& H_{2}\left(T_{2}\right) \stackrel{d_{1,2}^{1}}{\longleftarrow} H_{2}\left(T_{2}\right) \stackrel{d_{2,2}^{1}}{\longleftarrow} H_{2}\left(R^{\times}\right) \stackrel{d_{3,2}^{1}}{\longleftarrow} E_{3,2}^{1} \longleftarrow * \\
& R^{\times} \times R^{\times} \stackrel{d_{1,1}^{1}}{\longleftarrow} R^{\times} \times R^{\times} \stackrel{d_{2,1}^{1}}{\longleftarrow} R^{\times} \stackrel{d_{3,1}^{1}}{\longleftarrow} E_{3,1}^{1} \longleftarrow *
\end{aligned}
$$

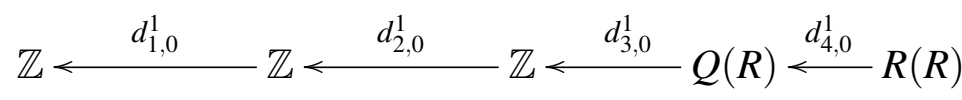

EXEMPLO 2.5. Estudaremos os diferenciais $d_{p, q}^{1}$ para $p=1,2,3$ e 4. Para isto, considere a resolução padrão $C_{\bullet}\left(G L_{2}(R)\right) \rightarrow \mathbb{Z}$ de $\mathbb{Z}$ sobre $G L_{2}(R)$.

- $d_{1, q}^{1}: H_{q}\left(T_{2}\right) \rightarrow H_{q}\left(T_{2}\right) \cong H_{q}\left(B_{2}\right):$ Note que

$$
\begin{aligned}
E_{0, q}^{1} & =H_{q}\left(C_{\bullet}\left(G L_{2}(R)\right) \otimes_{G L_{2}(R)} C_{0}\left(R^{2}\right)\right)=H_{q}\left(C_{\bullet}\left(G L_{2}(R)\right) \otimes_{B_{2}} \mathbb{Z}\right) \\
& \cong H_{q}\left(C_{\bullet}\left(G L_{2}(R)\right) \otimes_{T_{2}} \mathbb{Z}\right) \cong H_{q}\left(T_{2}\right), \\
E_{1, q}^{1} & =H_{q}\left(C_{\bullet}\left(G L_{2}(R)\right) \otimes_{G L_{2}(R)} C_{1}\left(R^{2}\right)\right)=H_{q}\left(C_{\bullet}\left(G L_{2}(R)\right) \otimes_{T_{2}} \mathbb{Z}\right) \cong H_{q}\left(T_{2}\right) .
\end{aligned}
$$

Usando o diagrama

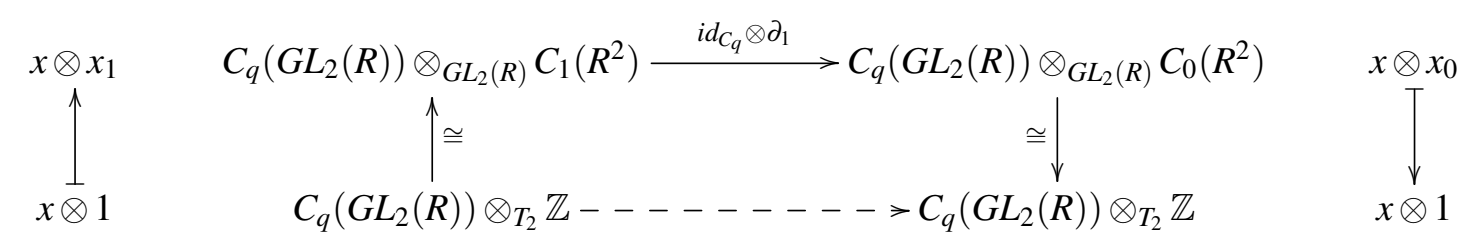

obtemos que

$$
\begin{aligned}
C_{q}\left(G L_{2}(R)\right) \otimes_{T_{2}} \mathbb{Z} & \longrightarrow C_{q}\left(G L_{2}(R)\right) \otimes_{T_{2}} \mathbb{Z}, \\
x \otimes 1 & \longmapsto(\omega x-x) \otimes 1=\omega x \otimes 1-x \otimes 1
\end{aligned}
$$


onde $\omega=\left(\begin{array}{ll}0 & 1 \\ 1 & 0\end{array}\right)$. Portanto pelo Exemplo (1.18) tomando $g_{0}=\omega$, temos

$$
d_{1, q}^{1}=H_{q}\left(\alpha_{\omega}\right)-H_{q}\left(i d_{T_{2}}\right)
$$

com $\alpha_{\omega}: T_{2} \rightarrow T_{2} \subseteq B_{2}$ definida como sendo $\alpha_{\omega}(a, b)=\omega\left(\begin{array}{ll}a & 0 \\ 0 & b\end{array}\right) \omega^{-1}=\left(\begin{array}{ll}b & 0 \\ 0 & a\end{array}\right)=$ $(b, a)$.

- $d_{2, q}^{1}: H_{q}\left(R^{\times}\right) \rightarrow H_{q}\left(T_{2}\right):$ Note que

$$
E_{2, q}^{1}=H_{q}\left(C_{\bullet}\left(G L_{2}(R)\right) \otimes_{G L_{2}(R)} C_{2}\left(R^{2}\right)\right)=H_{q}\left(C \bullet\left(G L_{2}(R)\right) \otimes_{R^{\times} I_{2}} \mathbb{Z}\right) \cong H_{q}\left(R^{\times}\right) .
$$

Usando o diagrama

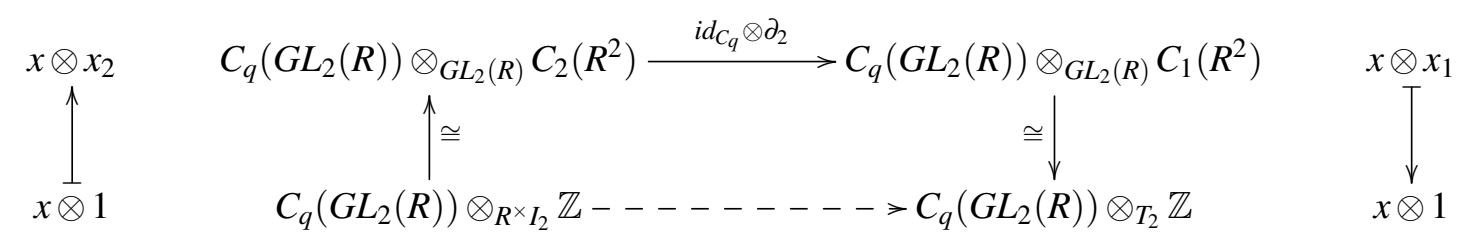

obtemos que

$$
\begin{aligned}
C_{q}\left(G L_{2}(R)\right) \otimes_{R^{\times} I_{2}} \mathbb{Z} & \longrightarrow C_{q}\left(G L_{2}(R)\right) \otimes_{T_{2}} \mathbb{Z}, \\
x \otimes 1 & \longmapsto\left(B^{\prime} \cdot x-B^{\prime \prime} \cdot x+I_{2} x\right) \otimes 1
\end{aligned}
$$

onde $B^{\prime}=\left(\begin{array}{ll}0 & 1 \\ 1 & 1\end{array}\right), B^{\prime \prime}=\left(\begin{array}{ll}1 & 1 \\ 0 & 1\end{array}\right)$. Pelo Exemplo (1.18)

$$
d_{2, q}^{1}=H_{q}\left(\alpha_{B^{\prime}}\right)-H_{q}\left(\alpha_{B^{\prime \prime}}\right)+H_{q}\left(\alpha_{I_{2}}\right)
$$

onde $\alpha_{B^{\prime}}, \alpha_{B^{\prime \prime}}, \alpha_{I_{2}}: R^{\times} \rightarrow T_{2}$ são definidas como $\alpha_{B^{\prime}}(a)=B^{\prime}\left(\begin{array}{ll}a & 0 \\ 0 & a\end{array}\right)\left(B^{\prime}\right)^{-1}=a I_{2}=(a, a)$, $\alpha_{B^{\prime \prime}}(a)=B^{\prime \prime}\left(\begin{array}{ll}a & 0 \\ 0 & a\end{array}\right)\left(B^{\prime \prime}\right)^{-1}=a I_{2}=(a, a), \alpha_{I_{2}}(a)=a I_{2}=(a, a)$. Portanto

$$
d_{2, q}^{1}=H_{q}(\Delta)-H_{q}(\Delta)+H_{q}(\Delta)=H_{q}(\Delta)
$$

onde $\Delta: R^{\times} \rightarrow T_{2}$ definida como $\Delta(a)=(a, a)$. É claro que $d_{2, q}^{1}$ é injetora.

- $d_{3, q}^{1}: \bigoplus_{a \in A} H_{q}\left(R^{\times}\right)[a] \rightarrow H_{q}\left(R^{\times}\right)$: Seja $a \in R^{\times}$tal que $1-a \in R^{\times}$. Note que

$$
\begin{aligned}
E_{3, q}^{1} & =H_{q}\left(C_{\bullet}\left(G L_{2}(R)\right) \otimes_{G L_{2}(R)} C_{3}\left(R^{2}\right)\right)=\bigoplus_{a \in A} H_{q}\left(C_{\bullet}\left(G L_{2}(R)\right) \otimes_{R^{\times} I_{2}} \mathbb{Z}\right)[a] \\
& \cong \bigoplus_{a \in A} H_{q}\left(R^{\times}\right)[a] .
\end{aligned}
$$


Usando o diagrama

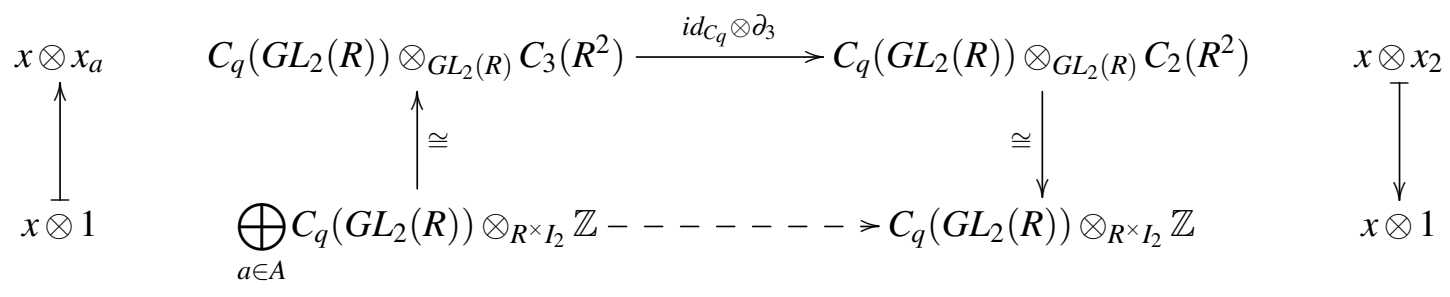

obtemos o homomorfismo

$$
\begin{aligned}
C_{q}\left(G L_{2}(R)\right) \otimes_{R^{\times} I_{2}} \mathbb{Z} & \longrightarrow C_{q}\left(G L_{2}(R)\right) \otimes_{R^{\times} I_{2}} \mathbb{Z}, \\
x_{a} \otimes 1 & \longmapsto\left(A_{a} \cdot x-B_{a} \cdot x+C_{a} \cdot x-x\right) \otimes 1
\end{aligned}
$$

onde $A_{a}=\left(\begin{array}{cc}0 & 1 \\ 1-a & 1\end{array}\right), B_{a}=\left(\begin{array}{cc}1-a & a \\ 0 & a\end{array}\right), C_{a}=\left(\begin{array}{cc}1 & 0 \\ 0 & a^{-1}\end{array}\right)$. Pelo Exemplo (1.18)

$$
d_{3, q}^{1}=H_{q}\left(\alpha_{A_{a}}\right)-H_{q}\left(\alpha_{B_{a}}\right)+H_{q}\left(\alpha_{C_{a}}\right)-H_{q}\left(\alpha_{i d_{R^{\times} I_{2}}}\right),
$$

onde $\alpha_{A_{a}}, \alpha_{B_{a}}, \alpha_{C_{a}}: R^{\times} \rightarrow R^{\times}$são definidas como $\alpha_{A_{a}}(x)=A_{a}\left(\begin{array}{ll}x & 0 \\ 0 & x\end{array}\right) A_{a}^{-1}=x I_{2}=x$,

$$
\begin{gathered}
\alpha_{B_{a}}(x)=B_{a}\left(\begin{array}{ll}
x & 0 \\
0 & x
\end{array}\right)\left(B_{a}\right)^{-1}=x I_{2}=x, \alpha_{C_{a}}(x)=C_{a}\left(\begin{array}{ll}
x & 0 \\
0 & x
\end{array}\right)\left(C_{a}\right)^{-1}=x I_{2}=x . \text { Assim } \\
d_{3, q}^{1}=H_{q}\left(i d_{R^{\times} I_{2}}\right)-H_{q}\left(i d_{R^{\times} I_{2}}\right)+H_{q}\left(i d_{R^{\times} I_{2}}\right)-H_{q}\left(i d_{R^{\times} I_{2}}\right)=0 .
\end{gathered}
$$

Portanto, $d_{3, q}^{1}=0$.

- $\bigoplus_{(a, b) \in B} H_{q}\left(R^{\times}\right)[a, b] \rightarrow \bigoplus_{a \in A} H_{q}\left(R^{\times}\right)[a]:$ Note que

$$
\begin{aligned}
E_{4, q}^{1} & =H_{q}\left(C_{\bullet}\left(G L_{2}(R)\right) \otimes_{G L_{2}(R)} C_{4}\left(R^{2}\right)\right)=\bigoplus_{(a, b) \in B} H_{q}\left(C_{\bullet}\left(G L_{2}(R)\right) \otimes_{R^{\times} I_{2}} \mathbb{Z}\right)[a, b], \\
& =\bigoplus_{(a, b) \in B} H_{q}\left(R^{\times}\right)[a, b]
\end{aligned}
$$

e $d_{4, q}^{1}$ é induzido pelo diagrama

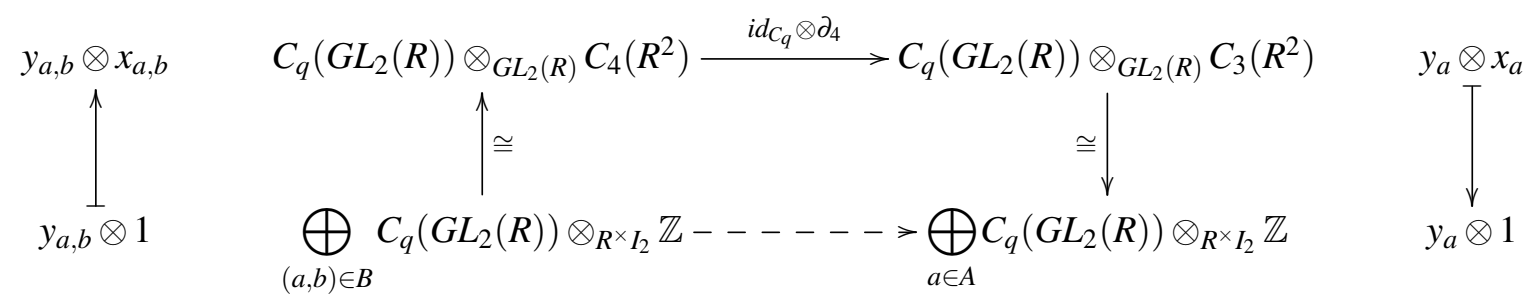

Agora por cálculo direto, temos

$$
y_{a, b} \otimes x_{a, b} \longmapsto y_{a, b} \otimes\left(A_{a, b} \cdot x_{\frac{1-a}{1-b}}-B_{a, b} \cdot x_{\frac{1-a^{-1}}{1-b^{-1}}}+C_{a, b} \cdot x_{\frac{b}{a}}-x_{b}+x_{a}\right),
$$


onde

$$
A_{a, b}=\left(\begin{array}{cc}
0 & \frac{1-b}{1-a} \\
-(1-b) & \frac{1-b}{1-a}
\end{array}\right), \quad B_{a, b}=\left(\begin{array}{cc}
1-b & -\frac{1-b}{1-a^{-1}} \\
0 & -\frac{1-b}{1-a^{-1}}
\end{array}\right), \quad C_{a, b}=\left(\begin{array}{ll}
1 & 0 \\
0 & a
\end{array}\right) .
$$

Portanto

$$
[a, b] \longmapsto\left[\frac{1-a}{1-b}\right]-\left[\frac{1-a^{-1}}{1-b^{-1}}\right]+\left[\frac{b}{a}\right]-[b]+[a]
$$

Em particular

$$
\begin{gathered}
d_{1,0}^{1}=0, \quad d_{2,0}^{1}=i d_{\mathbb{Z}}, \quad d_{3,0}^{1}=0 \\
d_{4,0}^{1}([a, b])=[a]-[b]+\left[\frac{b}{a}\right]-\left[\frac{1-a^{-1}}{1-b^{-1}}\right]+\left[\frac{1-a}{1-b}\right],
\end{gathered}
$$

$\mathrm{e}$

$$
\begin{aligned}
& d_{1,1}^{1}: R^{\times} \times R^{\times} \longrightarrow R^{\times} \times R^{\times}, \quad d_{2,1}^{1}: R^{\times} \longrightarrow R^{\times} \times R^{\times}, \quad d_{3,1}^{1}=0 . \\
& (a, b) \longmapsto\left(b a^{-1}, a b^{-1}\right) \quad a \longmapsto(a, a)
\end{aligned}
$$

Usando o isomorfismo $H_{2}\left(R^{\times}\right) \oplus H_{2}\left(R^{\times}\right) \oplus\left(R^{\times} \otimes_{\mathbb{Z}} R^{\times}\right) \cong H_{2}\left(T_{2}\right)$, temos

$$
\begin{aligned}
& H_{2}\left(R^{\times}\right) \longrightarrow H_{2}\left(T_{2}\right), \quad H_{2}\left(R^{\times}\right) \longrightarrow H_{2}\left(T_{2}\right), \\
& \mathbf{c}\left(a, a^{\prime}\right) \longmapsto \mathbf{c}\left((a, 1),\left(a^{\prime}, 1\right)\right) \quad \mathbf{c}\left(b, b^{\prime}\right) \longmapsto \mathbf{c}\left((1, b),\left(1, b^{\prime}\right)\right) \\
& \cup: H_{2}\left(R^{\times}\right) \longrightarrow H_{2}\left(T_{2}\right) . \\
& a \otimes b \longmapsto a \cup b:=\mathbf{c}((a, 1),(1, b))
\end{aligned}
$$

Agora é fácil verificar que

$$
d_{1,2}^{1}: H_{2}\left(R^{\times}\right) \oplus H_{2}\left(R^{\times}\right) \oplus\left(R^{\times} \otimes_{\mathbb{Z}} R^{\times}\right) \longrightarrow H_{2}\left(R^{\times}\right) \oplus H_{2}\left(R^{\times}\right) \oplus\left(R^{\times} \otimes_{\mathbb{Z}} R^{\times}\right)
$$

é dado por

$$
d_{1,2}^{1}(x, y, a \otimes b)=(y-x, x-y,-a \otimes b-b \otimes a) .
$$

Além disso,

$$
\begin{aligned}
d_{2,2}^{1}: H_{2}\left(R^{\times}\right) & \longrightarrow H_{2}\left(T_{2}\right)=H_{2}\left(R^{\times}\right) \oplus H_{2}\left(R^{\times}\right) \oplus\left(R^{\times} \otimes_{\mathbb{Z}} R^{\times}\right), \\
\mathbf{c}(a, b) & =\mathbf{c}((a, a),(b, b)) .
\end{aligned}
$$

Definimos o pre-grupo de Bloch do anel $R$, denotado $\mathfrak{p}(R)$, como o módulo quociente de $Q(R)$ por o submódulo gerado pelos elementos da forma $[a]-[b]+\left[\frac{b}{a}\right]-\left[\frac{1-a^{-1}}{1-b^{-1}}\right]+\left[\frac{1-a}{1-b}\right]$, onde $a, 1-a, b, 1-b, a-b \in R^{\times}$. Considere agora o homomorfismo

$$
\begin{aligned}
\sigma: R^{\times} \otimes_{\mathbb{Z}} R^{\times} & \longrightarrow R^{\times} \otimes_{\mathbb{Z}} R^{\times} \\
a \otimes b & \longmapsto-b \otimes a
\end{aligned}
$$


e defina os seguintes módulos

$$
\begin{aligned}
\left(R^{\times} \otimes_{\mathbb{Z}} R^{\times}\right)^{\sigma} & :=\{a \otimes b: \sigma(a \otimes b)=a \otimes b\}, \\
\left(R^{\times} \otimes_{\mathbb{Z}} R^{\times}\right)_{\sigma} & :=\frac{R^{\times} \otimes_{\mathbb{Z}} R^{\times}}{\left\langle\sigma(a \otimes b)-(a \otimes b): a, b \in R^{\times}\right\rangle}=\frac{R^{\times} \otimes_{\mathbb{Z}} R^{\times}}{\left\langle a \otimes b+b \otimes a: a, b \in R^{\times}\right\rangle} \\
H_{2}\left(T_{2}\right)^{\sigma} & :=\left\{(x, x, a \otimes b): x \in H_{2}\left(R^{\times}\right), \sigma(a \otimes b)=a \otimes b\right\}, \\
H_{2}\left(T_{2}\right)_{\sigma} & :=\frac{H_{2}\left(R^{\times}\right) \oplus H_{2}\left(R^{\times}\right) \oplus R^{\times} \otimes_{\mathbb{Z}} R^{\times}}{\left\langle(x,-x,-a \otimes b-b \otimes a): x \in H_{2}\left(R^{\times}\right), a, b, \in R^{\times}\right\rangle} \cong H_{2}\left(R^{\times}\right) \oplus\left(R^{\times} \otimes_{\mathbb{Z}} R^{\times}\right)_{\sigma} .
\end{aligned}
$$

O elemento de $\mathfrak{p}(R)$ representado por $[a]$ será denotado novamente por $[a]$ e o elemento de $\left(R^{\times} \otimes_{\mathbb{Z}} R^{\times}\right)_{\sigma}$ representado por $a \otimes b$ será denotado por $a \otimes b$. Logo a segunda e terceira folha da sequência espectral dada na Proposição (2.6) são da forma

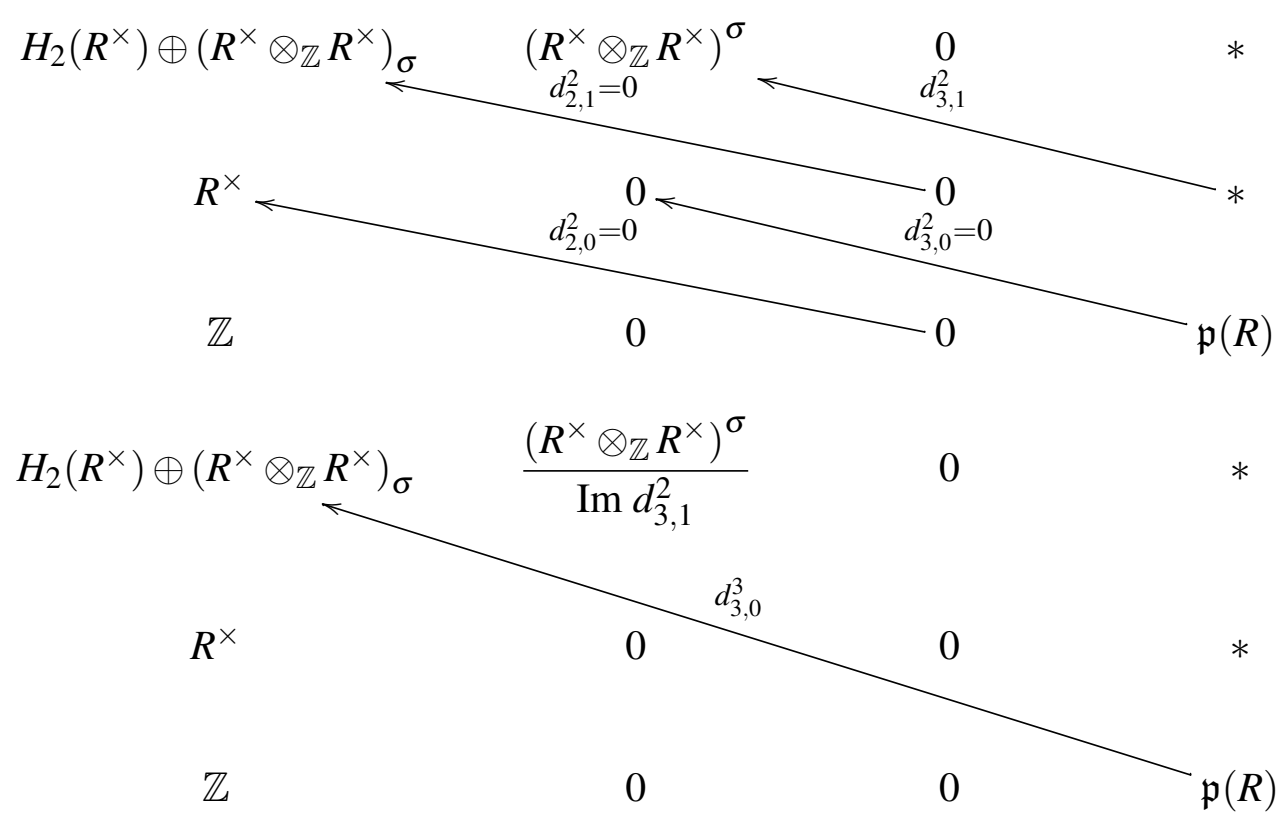

LEMA 2.8. O diferencial $d_{3,0}^{3}: \mathfrak{p}(R) \rightarrow H_{2}\left(R^{\times}\right) \oplus\left(R^{\times} \otimes_{\mathbb{Z}} R^{\times}\right)_{\sigma}$ é dado por

$$
d_{3,0}^{3}([a])=(a \wedge(1-a),-a \otimes(1-a)) .
$$

Demonstração. Considere o seguinte diagrama

$$
\begin{gathered}
C_{2}\left(G L_{2}(R)\right) \otimes C_{0}\left(R^{2}\right) \stackrel{i d C_{C_{2}} \otimes \partial_{1}}{\longleftarrow} C_{2}\left(G L_{2}(R)\right) \otimes C_{1}\left(R^{2}\right) \\
\delta_{2} \otimes i d_{C_{1}} \downarrow \\
C_{1}\left(G L_{2}(R)\right) \otimes C_{1}\left(R^{2}\right) \stackrel{i d d_{C_{1}} \otimes \partial_{2}}{\longrightarrow} C_{1}\left(G L_{2}(R)\right) \otimes C_{2}\left(R^{2}\right) \\
\delta_{1} \otimes i d_{C_{2}} \downarrow \\
C_{0}\left(G L_{2}(R)\right) \otimes C_{2}\left(R^{2}\right) \stackrel{i d C_{C_{0}} \otimes \partial_{3}}{=} C_{0}\left(G L_{2}(R)\right) \otimes C_{3}\left(R^{2}\right) .
\end{gathered}
$$

O elemento $[a] \in \mathfrak{p}(R)$ é representado pelo elemento $(1) \otimes x_{a} \in C_{0}\left(G L_{2}(R)\right) \otimes_{G L_{2}(R)} C_{3}\left(R^{2}\right)$. Pelo diagrama, o elemento $(1) \otimes x_{a} \in C_{0}\left(G L_{2}(R)\right) \otimes_{G L_{2}(R)} C_{3}\left(R^{2}\right)$ vai para o elemento

$$
\left[\left(g_{1}\right)-\left(g_{2}\right)+\left(g_{3}\right)-(1)\right] \otimes x_{2},
$$


onde $g_{1}=\left(\begin{array}{cc}0 & 1 \\ a-1 & 1\end{array}\right), g_{2}=\left(\begin{array}{cc}1-a & a \\ 0 & a\end{array}\right), g_{3}=\left(\begin{array}{ll}1 & 0 \\ 0 & a\end{array}\right)$. Note que o elemento

$$
y_{a}:=\left[\left(g_{2}, g_{1}\right)-\left(g_{3}, 1\right)\right] \in C_{1}\left(G L_{2}(R)\right)
$$

satisfaz

$$
\delta_{1} \otimes i d_{C_{2}}\left(y_{a} \otimes x_{2}\right)=\left[\left(g_{1}\right)-\left(g_{2}\right)+\left(g_{3}\right)-(1)\right] \otimes x_{2} .
$$

Agora dado que

$$
\left(a^{-1} g_{2}^{2} g_{3}^{2}, g_{1} g_{2}\right)=\left(g_{3} g_{2}, g_{3} g_{1}\right)\left(\begin{array}{cc}
a^{-1}(1-a) & 0 \\
0 & a
\end{array}\right)
$$

se

$$
\begin{aligned}
z_{a}:= & \left(g_{3} g_{1}, g_{2}, g_{1}\right)-\left(g_{3} g_{2}, g_{3} g_{1}, g_{2}\right)-\left(a^{-1} g_{2}^{2} g_{3}^{2}, g_{2}^{2}, g_{1} g_{2}\right) \\
& +\left(a^{-3} g_{2}^{2} g_{3}^{2}, a^{-2} g_{2}^{2}, 1\right)-\left(a^{-3} g_{2}^{2} g_{3}^{2}, a^{-1} g_{3}^{2}, 1\right) \\
& +\left(g_{1}^{2} g_{3}^{-1}, a^{-1} g_{1}^{2}, 1\right)-\left(g_{1}^{2} g_{3}^{-1}, a g_{3}^{-1}, 1\right)+\left(g_{3}, a^{-1} g_{3}^{2}, 1\right)
\end{aligned}
$$

então temos $\delta_{2} \otimes i d_{C_{1}}\left(z_{a} \otimes x_{1}\right)=i d_{C_{1}} \otimes \partial_{2}\left(y_{a} \otimes x_{2}\right)$. Logo o elemento $z_{a} \otimes x_{1}$ vai para o elemento $\left(z_{a} \omega-z_{a}\right) \otimes x_{0} \in C_{2}\left(G L_{2}(R)\right) \otimes_{G L_{2}(R)} C_{0}\left(R^{2}\right)$, onde $\omega=\left(\begin{array}{cc}0 & 1 \\ 1 & 0\end{array}\right)$. Note que $\left(z_{a} \omega-z_{a}\right) \otimes x_{0}$ é um representante do elemento $d_{3,0}^{3}([a])$. Vamos estudar esse elemento e para isso seja $s$ : $G L_{2}(R) / B_{2} \rightarrow G L_{2}(R)$ uma seção da projeção canônica $\pi: G L_{2}(R) \rightarrow G L_{2}(R) / B_{2}$, definida como sendo

$$
s\left(g B_{2}\right)= \begin{cases}1 & \text { se } g \cdot\left(\left\langle e_{1}\right\rangle\right)=\left(\left\langle e_{1}\right\rangle\right), \\
\omega & \text { se } g \cdot\left(\left\langle e_{1}\right\rangle\right)=\left(\left\langle e_{2}\right\rangle\right), \\
\left(\begin{array}{ll}
1 & 0 \\
x & 1
\end{array}\right) & \text { se } g \cdot\left(\left\langle e_{1}\right\rangle\right)=\left(\left\langle e_{1}+x e_{2}\right\rangle\right) .\end{cases}
$$

Para cada $g \in G L_{2}(R)$, defina

$$
\bar{g}:=(s \circ \pi(g))^{-1} g .
$$

Logo temos um homomorfismo de complexos de cadeias como foi dado no Exemplo (1.17)

$$
\begin{aligned}
\phi_{\bullet}: C_{\bullet}\left(G L_{2}(R)\right) \otimes_{G L_{2}(R)} C_{0}\left(R^{2}\right) & \longrightarrow C_{\bullet}\left(B_{2}\right) \otimes_{B_{2}} \mathbb{Z} \\
\left(g_{0}, g_{1}, \ldots, g_{n}\right) \otimes x_{0} & \longmapsto\left(\overline{g_{0}}, \overline{g_{1}}, \ldots, \overline{g_{n}}\right) \otimes 1
\end{aligned}
$$

que induz o isomorfismo

$$
H_{2}\left(G L_{2}(R), C_{0}\left(R^{2}\right)\right)=H_{2}\left(C_{2}\left(G L_{2}(R)\right) \otimes_{G L_{2}(R)} C_{0}\left(R^{2}\right)\right) \stackrel{\phi_{*}}{\longrightarrow} H_{2}\left(C_{2}\left(B_{2}\right) \otimes_{B_{2}} \mathbb{Z}\right)=H_{2}\left(B_{2}\right) .
$$

Considere o isomorfismo $\mathrm{H}_{2}\left(B_{2}\right) \stackrel{\alpha_{*}}{\longrightarrow} H_{2}\left(T_{2}\right)$ induzido pelo homomorfismo

$$
\begin{aligned}
\alpha: B_{2} & \longrightarrow T_{2} \\
\left(\begin{array}{ll}
a & c \\
0 & b
\end{array}\right) & \longmapsto(a, b)
\end{aligned}
$$


Usando o isomorfismo $H_{2}\left(T_{2}\right)=H_{2}\left(C_{\bullet}\left(T_{2}\right) \otimes_{T_{2}} \mathbb{Z}\right) \stackrel{\cong}{\longrightarrow} H_{2}\left(B_{\bullet}\left(T_{2}\right) \otimes_{T_{2}} \mathbb{Z}\right)=H_{2}\left(T_{2}\right)$, induzido pelo morfismo

$$
\begin{aligned}
\theta_{\bullet}: C_{\bullet}\left(T_{2}\right) & \longrightarrow B_{\bullet}\left(T_{2}\right) \\
\left(g_{0}, g_{1}, \ldots, g_{n}\right) & \longmapsto\left[g_{0} g_{1}^{-1}|\cdots| g_{n-1} g_{n}^{-1}\right]
\end{aligned}
$$

temos que o isomorfismo $H_{2}\left(G L_{2}(R), C_{0}\left(R^{2}\right)\right) \cong H_{2}\left(T_{2}\right)$ é induzido por

$$
C_{2}\left(G L_{2}(R)\right) \otimes_{G L_{2}(F)} C_{0}\left(R^{2}\right) \stackrel{\theta_{2} \circ \alpha_{2} \circ \phi_{2}}{\longrightarrow} B_{2}\left(T_{2}\right) \otimes_{T_{2}} \mathbb{Z} .
$$

Com uma cálculo direto (e um pouco complicado) podemos ver que esta aplicação leva $\left(z_{a} \omega-\right.$ $\left.z_{a}\right) \otimes x_{0}$ no elemento $u_{a} \otimes 1$, onde

$$
\begin{aligned}
u_{a}= & +\left[\left(a^{-1}, a\right) \mid(a, 1)\right]-\left[\left(-a, a^{-1}\right) \mid(-1, a)\right] \\
& +\left[\left(-a^{-1}, a^{2}\right) \mid\left(-a, a^{-1}\right)\right]-\left[(a, 1) \mid\left(a^{-1}, a\right)\right] \\
& +\left[\left(a^{-1}, a\right) \mid(-1, a)\right]-\left[\left(a, a^{-1}\right) \mid(1, a)\right] \\
& +\left[\left(a, a^{-1}\right) \mid\left(a^{-1},-a^{-1}(1-a)^{2}\right)\right]-\left[\left(a^{-1}, a\right) \mid\left(a^{-2}(1-a)^{2}, 1\right)\right] \\
& -\left[\left(a^{-1},-a^{-1}(1-a)^{2}\right) \mid\left(a, a^{-1}\right)\right]+\left[\left(a^{-2}(1-a)^{2}, 1\right) \mid\left(a^{-1}, a\right)\right] \\
& +\left[(1, a) \mid\left(a^{-1},-a^{-1}(1-a)^{2}\right)\right]-\left[(a, 1) \mid\left(-a^{-1}(1-a),-a^{-1}(1-a)\right)\right] \\
& -\left[\left(a^{-1},-a^{-1}(1-a)^{2}\right) \mid(1, a)\right]+\left[\left(-a^{-1}(1-a),-a^{-1}(1-a)\right) \mid(a, 1)\right] \\
& +\left[(1, a) \mid\left(a, a^{-1}\right)\right]-\left[(a, 1) \mid\left(a^{-1}, a\right)\right] .
\end{aligned}
$$

Nas homologias temos que $d_{3,0}^{3}: \mathfrak{p}(R) \rightarrow H_{2}\left(R^{\times}\right) \oplus\left(R^{\times} \otimes_{\mathbb{Z}} R^{\times}\right)_{\sigma}$ leva $[a]$ no elemento

$$
\begin{aligned}
& \mathbf{c}\left((-1, a),\left(-a, a^{-1}\right)\right)+2 \mathbf{c}\left(\left(a^{-1}, a\right),(a, 1)\right) \\
& +\mathbf{c}\left((1, a),\left(a, a^{-1}\right)\right)+\mathbf{c}\left(\left(a^{-2}(1-a)^{2}, 1\right),\left(a^{-1}, a\right)\right) \\
& +\mathbf{c}\left((a, 1),\left(a^{-1},-a^{-1}(1-a)^{2}\right)\right)+\mathbf{c}\left(\left(-a^{-1}(1-a),-a^{-1}(1-a)\right),(a, 1)\right),
\end{aligned}
$$

que é igual ao elemento $\mathbf{c}\left((a, 1),\left(1-a,(1-a)^{-1}\right)\right)$. Portanto

$$
\begin{aligned}
d_{3,0}^{3}: E_{3,0}^{3} \cong \mathfrak{p}(R) & \longrightarrow E_{0,2}^{3}=H_{2}\left(T_{2}\right)_{\sigma} \cong \bigwedge_{\mathbb{Z}}^{2} R^{\times} \oplus\left(R^{\times} \otimes_{\mathbb{Z}} R^{\times}\right)_{\sigma} \\
{[a] } & \longmapsto \mathbf{c}\left((a, 1),\left(1-a,(1-a)^{-1}\right)\right)=(a \wedge(1-a),-a \otimes(1-a))
\end{aligned}
$$

O Lema (2.8) implica que

$$
E_{0,2}^{4}=\frac{H_{2}\left(R^{\times}\right) \oplus\left(R^{\times} \otimes_{\mathbb{Z}} R^{\times}\right)_{\sigma}}{\operatorname{Im} d_{3,0}^{3}}=\frac{H_{2}\left(R^{\times}\right) \oplus\left(R^{\times} \otimes_{\mathbb{Z}} R^{\times}\right)_{\sigma}}{\left\langle(a \wedge(1-a),-a \otimes(1-a)): a, 1-a \in R^{\times}\right\rangle} .
$$

A sequência espectral dada na Proposição (2.6) dá-nos uma filtração de $H_{2}\left(G L_{2}(R)\right)$

$$
0=F_{-1} H_{2}\left(G L_{2}(R)\right) \subseteq F_{0} H_{2}\left(G L_{2}(R)\right) \subseteq F_{1} H_{2}\left(G L_{2}(R)\right) \subseteq F_{2} H_{2}\left(G L_{2}(R)\right)=H_{2}\left(G L_{2}(R)\right),
$$

tal que

$$
\begin{aligned}
& E_{0,2}^{4} \cong E_{0,2}^{\infty} \cong F_{0} H_{2}\left(G L_{2}(R)\right), \\
& E_{1,1}^{3} \cong E_{1,1}^{\infty} \cong \frac{F_{1} H_{2}\left(G L_{2}(R)\right)}{F_{0} H_{2}\left(G L_{2}(R)\right)}, \\
& E_{2,0}^{3} \cong E_{2,0}^{\infty} \cong \frac{H_{2}\left(G L_{2}(R)\right)}{F_{1} H_{2}\left(G L_{2}(R)\right)} .
\end{aligned}
$$


Como $E_{1,1}^{3}=E_{2,0}^{3}=0$, temos que $F_{0} H_{2}\left(G L_{2}(R)\right)=F_{1} H_{2}\left(G L_{2}(R)\right)=H_{2}\left(G L_{2}(R)\right)$ e portanto

$$
H_{2}\left(G L_{2}(R)\right) \cong E_{0,2}^{\infty} \cong \frac{H_{2}\left(R^{\times}\right) \oplus\left(R^{\times} \otimes_{\mathbb{Z}} R^{\times}\right)_{\sigma}}{\left\langle(a \wedge(1-a),-a \otimes(1-a)): a, 1-a \in R^{\times}\right\rangle}
$$

COROLÁRIO 2.2. O homomorfismo $H_{2}\left(T_{2}\right) \rightarrow H_{2}\left(G L_{2}(R)\right)$, induzido por $T_{2} \hookrightarrow G L_{2}(R)$ é sobrejetor.

Demonstração. Como $E_{0,2}^{\infty}$ é quociente de $H_{2}\left(T_{2}\right)$, o isomorfismo (2.6) implica que $H_{2}\left(T_{2}\right) \rightarrow$ $H_{2}\left(G L_{2}(R)\right)$ é sobrejetor.

Corolário 2.3. Seja $R$ um anel local com ideal maximal $\mathfrak{m}$ e seja $T_{3}:=\left\{\operatorname{diag}(a, b, c) \in G L_{3}(R): a, b, c \in R^{\times}\right\}$. Se $R / \mathfrak{m}$ é infinito, então as inclusões

$$
\begin{aligned}
\text { inc }_{1}: T_{3} & \longrightarrow G L_{3}(R), & \text { inc }: G L_{2} & \longrightarrow G L_{3}(R) \\
(a, b, c) & \longmapsto\left(\begin{array}{lll}
a & 0 & 0 \\
0 & b & 0 \\
0 & 0 & c
\end{array}\right) & A & \longmapsto\left(\begin{array}{cc}
A & 0 \\
0 & 1
\end{array}\right)
\end{aligned}
$$

induzem o homomorfismo sobrejetor

$$
H_{3}\left(T_{3}\right) \oplus H_{3}\left(G L_{2}(R)\right) \rightarrow H_{3}\left(G L_{3}(R)\right) .
$$

Demonstração. Pela fórmula de Künneth (Proposição 1.3), $H_{3}\left(R^{\times} \times G L_{2}(R)\right) \cong H_{3}\left(G L_{2}(R)\right) \oplus$ $M$, onde

$$
M \cong H_{3}\left(R^{\times}\right) \oplus\left(H_{2}\left(R^{\times}\right) \otimes H_{1}\left(G L_{2}(R)\right)\right) \oplus\left(H_{1}\left(R^{\times}\right) \otimes H_{2}\left(G L_{2}(R)\right)\right) \oplus \operatorname{Tor}_{1}^{\mathbb{Z}}\left(H_{1}\left(R^{\times}\right), H_{1}\left(G L_{2}(R)\right)\right) .
$$

Como pelo Teorema de Estabilidade (1.16), $H_{1}\left(G L_{1}(R)\right) \cong H_{1}\left(G L_{2}(R)\right)$ e com uso do Lema (1.5), é fácil verificar que esta decomposição é canônica. Note que

$$
\begin{aligned}
H_{3}\left(T_{3}\right) & =H_{3}\left(R^{\times} \times T_{2}\right) \\
& =H_{3}\left(R^{\times}\right) \oplus\left(H_{2}\left(R^{\times}\right) \otimes H_{1}\left(T_{2}\right)\right) \oplus\left(H_{1}\left(R^{\times}\right) \otimes H_{2}\left(T_{2}\right)\right) \oplus H_{3}\left(T_{2}\right) \oplus \operatorname{Tor}_{1}^{\mathbb{Z}}\left(H_{1}\left(R^{\times}\right), H_{1}\left(T_{2}\right)\right) .
\end{aligned}
$$

Como $H_{1}\left(G L_{1}(R)\right) \cong H_{1}\left(G L_{2}(R)\right)$, temos

$$
\begin{gathered}
H_{2}\left(R^{\times}\right) \otimes H_{1}\left(G L_{2}(R)\right) \cong H_{2}\left(R^{\times}\right) \otimes H_{1}\left(R^{\times}\right) \subseteq\left(\operatorname{inc}_{1}\right)_{*}\left(H_{3}\left(T_{3}\right)\right), \\
\operatorname{Tor}_{1}^{\mathbb{Z}}\left(H_{1}\left(R^{\times}\right), H_{1}\left(G L_{2}(R)\right)\right) \cong \operatorname{Tor}_{1}^{\mathbb{Z}}\left(H_{1}\left(R^{\times}\right), H_{1}\left(R^{\times}\right)\right) \subseteq\left(\text { inc }_{1}\right)_{*}\left(H_{3}\left(T_{3}\right)\right) .
\end{gathered}
$$

Além disso pelo Corolário (2.2), o homomorfismo $H_{1}\left(F^{\times}\right) \otimes H_{2}\left(T_{2}\right) \rightarrow H_{1}\left(F^{\times}\right) \otimes H_{2}\left(G L_{2}(F)\right)$ é sobrejetor. Dado o seguinte diagrama comutativo

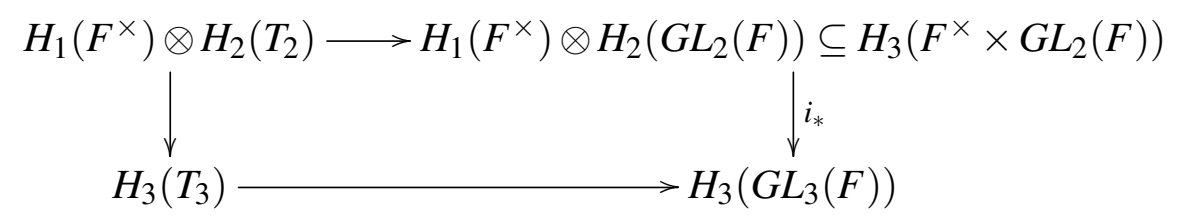

temos que $i_{*}\left(H_{1}\left(R^{\times}\right) \otimes H_{2}\left(G L_{2}(R)\right)\right) \subseteq(\text { inc })_{*}\left(H_{3}\left(T_{3}\right)\right)$. Portanto $i_{*}(M) \subseteq(\text { inc })_{*}\left(H_{3}\left(T_{3}\right)\right)$ e o resultado segue do Teorema de Estabilidade (1.16). 
TeOrema 2.2 (Teorema de Van der Kallen-Matsumoto). Seja $R$ um anel local com ideal maximal $\mathfrak{m}$ tal que $R / \mathfrak{m}$ é um corpo infinito. Então

$$
K_{2}(R) \cong K_{2}^{M}(R)
$$

onde $K_{2}^{M}(R)$ é o grupo abeliano aditivo gerado pelos símbolos $\{a, b\}$ com $a, b \in R^{\times}$satisfazendo para todo $a, a_{1}, a_{2}, b, b_{1}, b_{2}, c, 1-c \in R^{\times}$

$$
\left\{a_{1} a_{2}, b\right\}=\left\{a_{1}, b\right\}+\left\{a_{2}, b\right\}, \quad\left\{a, b_{1} b_{2}\right\}=\left\{a, b_{1}\right\}+\left\{a, b_{2}\right\}, \quad\{c, 1-c\}=0, \quad\{a, b\}=-\{b, a\} .
$$

Demonstração. Pelo Exemplo (2.3) temos que $E(R)=[S L(R), S L(R)]=S L(R)$. Agora pela decomposição (1.14) e o Teorema (2.1) temos

$$
H_{2}(G L(R))=H_{2}\left(R^{\times}\right) \oplus H_{2}(S L(R))=H_{2}\left(R^{\times}\right) \oplus H_{2}(E(R))=H_{2}\left(R^{\times}\right) \oplus K_{2}(R) .
$$

É fácil verificar que

$$
K_{2}^{M}(R) \cong \frac{R^{\times} \otimes_{\mathbb{Z}} R^{\times}}{\left\langle a \otimes(1-a), b \otimes c+c \otimes b: a, 1-a, b, c \in R^{\times}\right\rangle}
$$

Considere a sequência espectral que estudamos no Exemplo (2.5). Definimos os seguintes homomorfismos

$$
\begin{array}{rlrl}
f: H_{2}\left(R^{\times}\right) & \longrightarrow E_{0,2}^{\infty}, & g: E_{0,2}^{\infty} & \longrightarrow K_{2}^{M}(R), \\
x \longmapsto(x, 0) & (x, a \otimes b) & \longmapsto-\{a, b\} \\
\alpha: E_{0,2}^{\infty} \longrightarrow H_{2}\left(R^{\times}\right), & \beta: K_{2}^{M}(R) & \longrightarrow E_{0,2}^{\infty} . \\
(x, a \otimes b) & \{x+a \wedge b & \{a, b\} & \longmapsto(a \wedge b,-a \otimes b)
\end{array}
$$

Como

$$
\begin{gathered}
\alpha \circ f=i d_{H_{2}\left(R^{\times}\right)}, \quad f \circ \alpha+\beta \circ g=i d_{E_{0,2}^{\infty},}, \quad g \circ \beta=i d_{K_{2}^{M}(R)}, \\
g \circ f=0, \quad \alpha \circ \beta=0,
\end{gathered}
$$

obtemos assim uma sequência exata que cinde

$$
0 \longrightarrow H_{2}\left(R^{\times}\right) \stackrel{f}{\longrightarrow} E_{0,2}^{\infty} \stackrel{g}{\longrightarrow} K_{2}^{M}(R) \longrightarrow 0 .
$$

Logo segue a seguinte decomposição

$$
H_{2}\left(G L_{2}(R)\right)=E_{0,2}^{\infty} \cong H_{2}\left(R^{\times}\right) \oplus K_{2}^{M}(R) .
$$

Finalmente o resultado é dado pelo Teorema de Estabilidade (1.16) para $G L_{2}(R)$

$$
H_{2}\left(G L_{2}(R)\right) \cong H_{2}(G L(R))
$$

junto com as decomposições feitas em (2.7) e (2.8). 
OBSERVAÇÃo 2.1. Detalharemos o homomorfismo $\beta: K_{2}^{M}(R) \rightarrow E_{0,2}^{\infty} \cong H_{2}(G L(R))$. Para isto note que

$$
\begin{aligned}
\beta(\{a, b\}) & =(a \wedge b,-a \otimes b)=\mathbf{c}\left(\operatorname{diag}(a, 1), \operatorname{diag}\left(b, b^{-1}\right)\right) \in H_{2}\left(G L_{2}(R)\right) \\
& =\mathbf{c}\left(\operatorname{diag}(a, 1,1), \operatorname{diag}\left(b, b^{-1}, 1\right)\right) \in H_{2}(G L(R)) \\
& =\mathbf{c}\left(\operatorname{diag}\left(a, 1, a^{-1}\right), \operatorname{diag}\left(b, b^{-1}, 1\right)\right)+\mathbf{c}\left(\operatorname{diag}(1,1, a), \operatorname{diag}\left(b, b^{-1}, 1\right)\right) \\
& =\mathbf{c}\left(\operatorname{diag}\left(a, 1, a^{-1}\right), \operatorname{diag}\left(b, b^{-1}, 1\right)\right)+\mathbf{c}(\operatorname{diag}(1,1, a), \operatorname{diag}(b, 1,1)) \\
& -\mathbf{c}(\operatorname{diag}(1,1, a), \operatorname{diag}(1, b, 1)) \\
& =\mathbf{c}\left(\operatorname{diag}\left(a, 1, a^{-1}\right), \operatorname{diag}\left(b, b^{-1}, 1\right)\right) \in H_{2}(S L(R))
\end{aligned}
$$

Portanto o isomorfismo

$$
K_{2}^{M}(R) \longrightarrow K_{2}(R)=H_{2}(S L(R))
$$

é dado por $\{a, b\} \mapsto \mathbf{c}\left(\operatorname{diag}\left(a, 1, a^{-1}\right), \operatorname{diag}\left(b, b^{-1}, 1\right)\right)$. Isto também prova que o isomorfismo obtido na demonstração do Teorema anterior (2.8) leva $K_{2}^{M}(R)$ no $K_{2}$-grupo do anel $R$.

OBSERVAÇÃo 2.2. No próximo capítulo no Lema (3.1), demonstraremos que na verdade

$$
K_{2}^{M}(R) \cong \frac{R^{\times} \otimes_{\mathbb{Z}} R^{\times}}{\left\langle a \otimes(1-a): a, 1-a \in R^{\times}\right\rangle}
$$

quando $R$ for um anel local com corpo residual infinito. 


\section{$K$-TEORIA ALGÉBRICA DE ANÉIS}

Neste capítulo primeiro apresentamos algumas definições importantes da topologia algébrica como os $C W$-complexos, os grupos de homologia e os grupos de homotopia de espaços topológicos, o espaço classificante de um grupo, etc. Depois definimos a +- construção de um espaço topológico e usamos isso para definir e estudar os $K$-grupos de anéis. No final, definimos os $K$-grupos de Milnor e comparamos eles com os $K$-grupos de anéis locais.

\section{1 $C W$-complexos}

Sejam $X, Y$ espaços topológicos, $A$ um subespaço fechado de $X$ e seja $\varphi: A \rightarrow Y$ uma aplicação contínua. Definimos o espaço adjunto de $X$ e $Y$ com respeito a $f$, denotado $Y \cup_{\varphi} X$, como sendo $Y \cup_{\varphi} X:=(Y \bigsqcup X) / \sim$, onde $Y \bigsqcup X$ é a união disjunta de $X$ e $Y$ e " $\sim$ " é uma relação de equivalência sobre $Y \bigsqcup X$ tal que $a \sim f(a)$ para todo $a \in A$. O espaço adjunto forma parte do seguinte diagrama comutativo

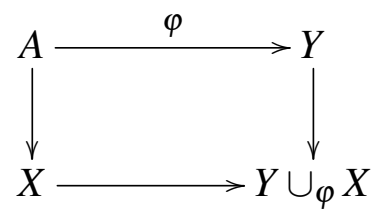

e contém $Y$ como subespaço fechado com aplicação injetiva $Y \rightarrow Y \cup_{\varphi} X, y \rightarrow \bar{y}$ e $Y$ tem complementar homeomorfo a $X-A$. Desta maneira, uma aplicação contínua $Y \cup_{\varphi} X \rightarrow Z$ é decomposta como uma aplicação contínua $h: Y \rightarrow Z$ e uma extensão $g: X \rightarrow Z$ da composta $h \circ \varphi: A \rightarrow Z$.

Seja $X$ um espaço topológico. Seja $n \geq 1$ e seja $\varphi=\bigsqcup_{\alpha \in \mathscr{A}} \varphi_{\alpha}: \bigsqcup_{\alpha \in \mathscr{A}} S_{\alpha}^{n-1} \rightarrow X$ uma aplicação contínua, onde $\bigsqcup_{\alpha \in \mathscr{A}} S_{\alpha}^{n-1}$ é a união disjunta de esferas de dimensão $n-1, S_{\alpha}^{n-1} \subseteq D_{\alpha}^{n}, \alpha \in \mathscr{A}$ e $D_{\alpha}^{n}$ é o disco unitário $n$-dimensional. Definimos a $n$-ésima extensão celular de $X$ com aplicação de colagem $\varphi$ e aplicação característica $\Phi$ como sendo o espaço adjunto $X \cup_{\varphi}\left(\bigsqcup_{\alpha} D_{\alpha}^{n}\right)$. A 
n-ésima extensão celular de $X$ forma parte do seguinte diagrama comutativo

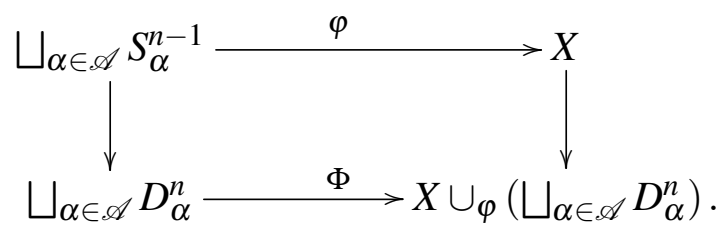

Note que a $n$-ésima extensão celular contém o espaço $X$ como subespaço fechado e contém como subespaços $n$-células abertas $e_{\alpha}^{n}:=\Phi\left(D_{\alpha}^{n}-S_{\alpha}^{n-1}\right), \alpha \in \mathscr{A}$ entendidas como $n$-ésimas bolas abertas. Note que

$$
\bigsqcup_{\alpha \in \mathscr{A}} e_{\alpha}^{n}=\left(X \cup_{\varphi}\left(\bigsqcup_{\alpha \in \mathscr{A}} D_{\alpha}^{n}\right)\right)-X
$$

isto é, as $n$-células abertas são as componentes conexas do complementar do espaço $X$. Em 1949 J.H.C. Whitehead introduziu a definição dos espaços $C W$-complexos de forma indutiva. Um espaço $C W$-complexo é um espaço topológico $X$ junto com uma filtração ascendente de subespaços

$$
\emptyset=X^{-1} \subseteq X^{0} \subseteq X^{1} \subseteq \cdots \subseteq X^{n-1} \subseteq X^{n} \subseteq \cdots \subseteq X=\bigcup_{n=-1}^{\infty} X^{n}
$$

tais que

i) o subespaço $X^{0}$ é um espaço topológico discreto,

ii) para todo $n \geq 1, X^{n}$ é homeomorfo à uma $n$-ésima extensão celular de $X^{n-1}$,

iii) a topologia de $X$ é coherente com a filtração anterior, isto é um subconjunto $U \subseteq X$ é aberto (ou fechado resp.) em $X$ se e somente se $U \cap X^{n}$ é aberto (ou fechado resp.) em $X^{n}$ para todo $n$.

Note-se que o segundo item implica que para todo $n \geq 1$ existem aplicações colagem $\varphi_{\alpha}: S^{n-1} \rightarrow$ $X^{n-1}$ e aplicações característica $\Phi_{\alpha}: D^{n} \rightarrow X^{n}$, para todo $\alpha \in \mathscr{A}_{n}$, tais que

- $X^{n} \cong X^{n-1} \bigcup_{\sqcup_{\alpha \in \mathscr{A}} \varphi_{\alpha}}\left(\bigsqcup_{\alpha \in \mathscr{A}} D_{\alpha}^{n}\right)$ e $X^{n-1}$ é um subespaço topológico fechado de $X^{n}$ para todo $n$,

- o complementar de $X^{n-1}$ em $X^{n}$ está dado por

$$
X^{n}-X^{n-1}=\bigsqcup_{\alpha \in \mathscr{A}_{n}} \Phi_{\alpha}\left(\operatorname{Int} D^{n}\right),
$$

onde $\Phi_{\alpha}\left(\right.$ Int $\left.D^{n}\right)$ são as $n$-ésimas células abertas de $X$.

Desta maneira o $C W$-complexo (como conjunto) pode ser expressado como a união disjunta

$$
X=\bigcup_{n=-1}^{\infty} X^{n}=\bigcup_{n=0}^{\infty}\left(X^{n}-X^{n-1}\right)=\bigsqcup_{n=0}^{\infty} \bigsqcup_{\alpha \in \mathscr{A}_{n}} \Phi_{\alpha}\left(\operatorname{Int} D^{n}\right)
$$


O objectivo do terceiro item da definição é munir a união de todos os espaços $X^{n}$ com a maior topologia de $X$ que torna todas as inclusões $X^{n} \rightarrow X$ aplicações contínuas.

Dizemos que um $C W$-complexo $X$ é de dimensão finita se $X=X^{n}$ para algum $n$. Caso contrário dizemos que $X$ é de dimensão infinita.

EXEMPLO 3.1. Os pontos da $n$-ésima esfera $S^{n} \subset \mathbb{R}^{n+1}$ tem coordenadas da forma $(x, u)$ onde $x \in \mathbb{R}^{n}$ e $u \in \mathbb{R}$. Sejam $D_{ \pm}^{n}$ as imagens dos mergulhos $D^{n} \rightarrow S^{n}, x \mapsto\left(x, \pm \sqrt{1-|x|^{2}}\right)$. Então

$$
S^{n}=S^{n-1} \cup D_{+}^{n} \cup D_{-}^{n}=S^{n-1} \cup_{\left(\operatorname{Id}_{S^{n-1}} \sqcup \mathrm{Id}_{S^{n-1}}\right)}\left(D^{n} \bigsqcup D^{n}\right),
$$

isto é, $S^{n}$ é obtida de $S^{n-1}$ colando duas $n$-células. Portanto indutivamente, a esfera infinita $S^{\infty}$

$$
S^{\infty}:=\bigcup_{n=0}^{\infty} S^{n}
$$

é um $C W$-complexo de dimensão infinita

$$
\emptyset=S^{-1} \subset S^{0} \subset S^{1} \subset S^{2} \subset \cdots \subset S^{n-1} \subset S^{n} \subset \cdots \subset S^{\infty} .
$$

Note que um subespaço $A \subset S^{\infty}$ é fechado se e somente se $A \cap S^{n}$ é um subespaço fechado de $S^{n}$ para todo $n \geq 0$.

EXEMPLO 3.2. O $n$-ésimo espaço projetivo real $\mathbb{R} P^{n}$ é definido como sendo o espaço de todas as retas em $\mathbb{R}^{n+1}$ que passam pelo origem. Cada uma destas retas é determinada por um único vetor em $\mathbb{R}^{n+1}$, a menos de multiplicação por escalares. Logo $\mathbb{R} P^{n}$ pode ser entendido como o espaço quociente do espaço $\mathbb{R}^{n+1}-\{0\}$ pela relação de equivalência $v \sim \lambda v$ para escalares $\lambda \in \mathbb{R}-\{0\}$. Restringindo $v$ para vetores de comprimento 1 , entendemos $\mathbb{R} P^{n}$ como o espaço quociente $S^{n} /(v \sim-v)$ com aplicação quociente $\pi_{n}: S^{n} \rightarrow \mathbb{R} P^{n}$. Isto é equivalente a dizer que $\mathbb{R} P^{n}$ é o espaço quociente de um hemisfério de $D^{n}$ com pontos antipodais de $\partial D^{n}$ identificados. Agora dado que $\partial D^{n}=S^{n-1}$ com pontos antipodais identificados é $\mathbb{R} P^{n-1}$, temos que $\mathbb{R} P^{n}$ é obtido de $\mathbb{R} P^{n-1}$ colando uma $n$-célula com aplicação de colagem $\pi_{n-1}: S^{n-1} \rightarrow \mathbb{R} P^{n-1}$ :

$$
\mathbb{R} P^{n}=\mathbb{R} P^{n-1} \cup_{\pi_{n-1}} D^{n}
$$

Portanto, $\mathbb{R} P^{n}$ é um $C W$-complexo de dimensão $n$ com uma célula em cada dimensão entre 0 e $n$. Em particular, $\mathbb{R} P^{0} \cong * \mathrm{e} \mathbb{R} P^{1} \cong S^{1}$. A união infinita $\mathbb{R} P^{\infty}=\bigcup_{n=0}^{\infty} \mathbb{R} P^{n}$ é um espaço $C W$-complexo de dimensão infinita com uma célula em cada dimensão.

Pode-se provar que todo $C W$-complexo é um espaco topológico normal (em particular é um espaço Hausdorff) [2, Proposição A.3]. Além disso, qualquer subespaço compacto num $C W$ complexo $X$ está contido em $X^{n}$ para algum $n \geq 0$ [2, Proposição A.1]. Também mostra-se que os $C W$-complexos são localmente contráteis e portanto localmente conexos por caminhos [2, Proposição A.4]. Consequentemente as definições de conexidade e conexidade por caminhos 
coincidem sobre os $C W$-complexos. Além disso, uma aplicação $f: X \rightarrow Y$ entre $C W$-complexos é dita celular se respeita a filtração, isto é $f\left(X^{n}\right) \subseteq Y^{n}$ para todo $n \geq 0$.

Um subcomplexo de um $C W$-complexo é um subespaço fechado que é união de células abertas. Note que o fecho das $n$-células abertas $e_{\alpha}^{n}:=\Phi\left(\operatorname{Int} D^{n}\right)$ está dado por $\overline{e_{\alpha}^{n}}=\Phi\left(D^{n}\right)$. Uma aplicação $f: X \rightarrow Y$ onde $X$ é um $C W$-complexo, é contínua se e somente se $\left.f\right|_{e_{\alpha}^{n}}$ são contínuas para todas as $n$-células abertas $e_{\alpha}^{n}, \alpha \in \mathscr{A}$ [2, Apêndice pág. 523]. Isto justifica o nome destes espaços já que o nome $C W$ representa as duas propriedades mais importantes dos $C W$-complexos, a saber $C$ : Closure-finiteness (ou fechamento finito) devido a que o fecho de cada célula aberta intercepta somente um número finito de outras células abertas; e $W$ : Weak topology (ou topologia fraca) devido a que um conjunto é fechado se, e somente se, intercepta o fecho de cada célula aberta num conjunto fechado.

Um espaço topológico $X$ é dito gerado por uma coleção de subespaços $X_{\alpha}$ se $X=\bigcup_{\alpha \in \mathscr{A}} X_{\alpha} \mathrm{e}$ qualquer conjunto $A \subseteq X$ é fechado em $X$ se, e somente se, $A \cap X_{\alpha}$ é fechado em $X_{\alpha}$ para cada $\alpha \in \mathscr{A}$. Desta forma, um $C W$-complexo é gerado pela coleção dos subespaços $\overline{e_{\alpha}^{n}}$ de todas suas $n$-células abertas $e_{\alpha}^{n}$. Como $X$ também é gerado por seus subespaços $X^{n}$ isto implica que $X$ é gerado por seus subespaços compactos, isto é todo $C W$-complexo é compactamente gerado [2, Apêndice pág. 523].

Seja $A$ um espaço topológico. Um espaço $C W$-complexo relativo a $A$ é um espaço topológico $X$ junto com uma filtração ascendente de subespaços

$$
A=X^{-1} \subset X^{0} \subset X^{1} \subset \cdots \subset X^{n-1} \subset X^{n} \subset \cdots \subset X=\bigcup_{n=-1}^{\infty} X^{n}
$$

tais que

i) o subespaço $X^{0}$ é a união de $A$ e um espaço topológico discreto,

ii) para todo $n \geq 1, X^{n}$ é homeomorfo à $n$-ésima extensão celular de $X^{n-1}$,

iii) a topologia de $X$ é coherente com a filtração anterior, isto é, um subconjunto $U \subseteq X$ é aberto (ou fechado resp.) em $X$ se e somente se $U \cap X^{n}$ é aberto (ou fechado resp.) em $X^{n}$ para todo $n$.

Denotaremos o $C W$ - complexos $X$ relativo a $A$ como sendo o par $(X, A)$. Note que se $A$ é um subcomplexo de um $C W$-complexo $X$, então $A$ é um $C W$-complexo com decomposição $A^{n}=A \cap X^{n}$. Além disso, o par $(X, A)$ é um espaço $C W$ - complexo relativo a $A$, e o espaço quociente $X / A$ é um espaço $C W$-complexo [2, Capítulo 0, pág. 8].

Na continuação apresentamos algumas ferramentas da topologia algébrica que usaremos neste capítulo. Sejam $f, g: X \rightarrow Y$ funções contínuas. Dizemos que $f$ e $g$ são homotópicas se existe uma 
função contínua (chamada homotopia) $F: X \times[0,1] \rightarrow Y$ tal que $F(x, 0)=f(x)$ e $F(x, 1)=g(x)$ para todo $x \in X$. No caso afirmativo escrevemos $f \simeq g$. Seja $A \subseteq X$ um subespaọ topológico de $X$ e seja $F: X \times[0,1] \rightarrow Y$ uma homotopia entre funções contínuas $f, g: X \rightarrow Y$. Se $\left.f\right|_{A}=\left.g\right|_{A}=$ $\left.F\right|_{A \times[0,1]}$, então dizemos que $F$ é uma homotopia relativa à $A$ e escrevemos $f \simeq_{A} g$. Dizemos que o espaço $X$ é contrátil se $i d_{X} \simeq c$, onde $c: X \rightarrow X$ é alguma função constante $c(x)=x_{0}$, para todo $x \in X$. Uma aplicação contínua $f: X \rightarrow Y$ é dita uma equivalência homotópica se existe uma aplicação contínua $g: Y \rightarrow X$ tal que $f \circ g \simeq i d_{Y}$ e $g \circ f \simeq i d_{X}$. Neste caso os espaços são chamados homotópicamente equivalentes ou que têm o mesmo tipo de homotopia e são denotamos por $X \simeq Y$.

Um $p$-simplexo $\Delta_{p}$ em $\mathbb{R}^{p+1}$ para $p \geq 0$ é o subconjunto

$$
\Delta_{p}:=\left\{\left(t_{0}, t_{1}, \ldots, t_{p}\right) \in \mathbb{R}^{n+1}: \sum_{i=0}^{p} t_{i}=1,0 \leq t_{i} \leq 1, i=0,1, \ldots, p\right\}
$$

Dado um espaço topológico $X$, um $p$-simplexo singular em $X$ é uma função $\phi: \Delta_{p} \rightarrow X$. Para um inteiro $0 \leq i \leq p$, definimos a $i$-ésima face de $\phi$, denotada $d_{i} \phi$, como sendo o $(p-1)$ simplexo singular em $X$ dado por $d_{i} \phi\left(t_{0}, \ldots, t_{p-1}\right)=\phi\left(t_{0}, \ldots, t_{i-1}, 0, t_{i}, \ldots, t_{p-1}\right)$. Denotamos por $S_{n}(X)$ o grupo abeliano livre cuja base é o conjunto de todos os $n$-simplexos singulares em $X$. Definimos a aplicação bordo $\partial_{n}: S_{n}(X) \rightarrow S_{n-1}(X)$ como sendo o homomorfismo definido por $\partial_{n}=\sum_{i=0}^{n}(-1)^{i} d_{i}$. É fácil verificar que $\left(S_{\bullet}(X), \partial_{\bullet}\right)$

$$
S_{\bullet}(X): \quad \cdots \rightarrow S_{n}(X) \stackrel{\partial_{n}}{\longrightarrow} S_{n-1}(X) \longrightarrow \cdots \longrightarrow S_{1}(X) \stackrel{\partial_{1}}{\longrightarrow} S_{0}(X) \rightarrow 0,
$$

é um complexo de cadeias. Definimos o $n$-ésimo grupo de homologia singular de $X$ com coeficientes em $\mathbb{Z}$, denotado $H_{n}(X, \mathbb{Z})$, como sendo

$$
H_{n}(X, \mathbb{Z}):=H_{n}\left(S_{\bullet}(X)\right) .
$$

EXEMPLO 3.3. Se $X \neq \emptyset$ e é conexo por caminhos, então $H_{0}(X, \mathbb{Z}) \cong \mathbb{Z}$. Para $n>0$, usando a sequência de Mayer-Vietoris [2, Exemplo 2.46], podemos mostrar que $H_{n}\left(S^{n}, \mathbb{Z}\right) \cong \mathbb{Z}$ e $H_{k}\left(S^{n}, \mathbb{Z}\right)=0$ para $k \neq n$ e $k \neq 0$.

Proposição 3.1. Seja $X$ um espaço topológico. Se $\left\{X_{\alpha}: \alpha \in I\right\}$ são as componentes conexas por caminho de $X$, então para todo $n \geq 0$,

$$
H_{n}(X, \mathbb{Z}) \cong \bigoplus_{\alpha \in I} H_{n}\left(X_{\alpha}, \mathbb{Z}\right)
$$

Demonstração. Veja-se [2, Proposição 2.6]

Se $(X, A)$ é um par de espaços topológicos com $A \subseteq X$, então $S_{\bullet}(A)$ é um subcomplexo de $S_{\bullet}(X)$. Além disso, dado que $\partial_{n}\left(S_{n}(A)\right) \subseteq S_{n-1}(A)$, definimos para todo $n \geq 0$ o grupo quociente

$$
S_{n}(X, A):=\frac{S_{n}(X)}{S_{n}(A)}
$$


É fácil verificar que $\left(S_{\bullet}(X, A), \bar{\partial}_{\bullet}\right)$ é um complexo de cadeias e definimos o $n$-ésimo grupo de homologia singular de $X$ relativo de $A$ como sendo

$$
H_{n}(X, A, \mathbb{Z}):=H_{n}\left(S_{\bullet}(X, A)\right)
$$

Note que $H_{n}(X, \mathbb{Z})=H_{n}(X, \emptyset, \mathbb{Z})$.

Exemplo 3.4. Seja $D^{n} \subseteq \mathbb{R}^{n}$ o disco unitário de dimensão $n$. Segundo [2, Exemplo 2.17], temos

$$
H_{i}\left(D^{n}, \partial D^{n}, \mathbb{Z}\right) \cong \begin{cases}\mathbb{Z} & \text { se } i=n \\ 0 & \text { se } i \neq n\end{cases}
$$

Seja $G$ um grupo abeliano e seja $(X, A)$ um par de espaços topológicos. Para todo $n \geq 0$, definimos

$$
S_{n}(X, A, G):=S_{n}(X, A) \otimes_{\mathbb{Z}} G
$$

$\mathrm{e}$

$$
\partial_{n}^{\prime}:=\partial_{n} \otimes i d_{G}
$$

Claramente $\left(S_{\bullet}(X, A, G), \partial_{\bullet}^{\prime}\right)$ é um complexo de cadeias e definimos a homologia singular de $X$ relativa de $A$ com coeficientes em $G$ como sendo

$$
H_{n}(X, A, G):=H_{n}\left(S_{\bullet}(X, A, G)\right)
$$

Seja $X$ um espaço topológico e seja $x_{0} \in X$ (chamado ponto base). Para $n \geq 1$, definimos o $n$-ésimo grupo de homotopia de $X$ respeito ao ponto base $x_{0}$, denotado $\pi_{n}\left(X, x_{0}\right)$, como sendo o grupo de todas as classes de homotopia $f: I^{n} \rightarrow X$ tal que $f\left(\partial I^{n}\right)=x_{0}$, onde $I^{n}=[0,1]^{n} \subseteq \mathbb{R}^{n} \mathrm{e}$ $\partial I^{n}$ é o bordo de $I^{n}$, com a operação soma + definida sobre as classes de homotopia como sendo $[f]+[g]:=[h] \in \pi_{n}\left(X, x_{0}\right)$, onde

$$
h\left(x_{1}, x_{2}, \ldots, x_{n}\right):= \begin{cases}f\left(2 x_{1}, x_{2}, \ldots, x_{n}\right) & \text { se } x_{1} \in\left[0, \frac{1}{2}\right] \\ g\left(2 x_{1}-1, x_{2}, \ldots, x_{n}\right) & \text { se } x_{1} \in\left[\frac{1}{2}, 1\right] .\end{cases}
$$

para todo $[f],[g] \in \pi_{n}\left(X, x_{0}\right)$. Para $n=0$, consideramos $\pi_{0}\left(X, x_{0}\right)$ como sendo o conjunto das componentes conexas por caminhos do espaço $X$. Quando o ponto base $x_{0}$ é conhecido denotamos $\pi_{n}\left(X, x_{0}\right)$ simplesmente por $\pi_{n}(X)$. Não é difícil demonstrar que $\pi_{n}\left(X, x_{0}\right)$ são grupos abelianos para $n \geq 2$ [2, Capítulo 4].

Um espaço $X$ é dito 0 -conexo se é conexo por caminhos, é dito 1-conexo se é conexo por caminhos e $\pi_{1}\left(X, x_{0}\right)=0$ para qualquer ponto $x_{0} \in X$. Em geral, $X$ é dito $n$-conexo se é conexo por caminhos e $\pi_{j}\left(X, x_{0}\right)=0$ para todo $j \leq n$ e para todo $x_{0} \in X$. Pode-se provar que se $X$ é um $C W$-complexo com células somente de dimensão maior ou igual a $n(n \geq 1)$, então $X$ é $(n-1)$-conexo e o homomorfismo induzido pela inclusão $\pi_{n}\left(X^{k}\right) \rightarrow \pi_{n}(X)$ é sobrejetivo se $k=n$ e é um isomorfismo se $k>n$ [8, Teorema 5.1.10, Corolário 5.1.11]. 
EXEMPLO 3.5. Dado que a esfera unitária de dimensão $n, S^{n} \subseteq \mathbb{R}^{n+1}$, como $C W$-complexo tem uma estrutura celular formada por duas células: $e^{0}$ e $e^{n}$. Então $S^{n}$ é $(n-1)$-conexo e portanto $\pi_{j}\left(S^{n}\right)=0$ para todo $j<n$.

Em seguida, apresentaremos os teoremas mais importantes dos espaços $C W$-complexos que permitem a definição do espaço classificante.

TEOREMA 3.1 (Whitehead). Um espaço $C W$-complexo $X$ é contrátil se e somente se $X$ é conexo e $\pi_{n}\left(X, x_{0}\right)=0$ para todo $n$ e para quaisquer $x_{0} \in X$. Uma aplicação $f: X \rightarrow Y$ entre espaços $C W$-complexos é uma equivalência homotópica se e somente se $f$ induz um isomorfismo $\pi_{n}\left(X, x_{0}\right) \stackrel{\cong}{\longrightarrow} \pi_{n}\left(Y, f\left(x_{0}\right)\right)$ para todo $x_{0} \in X$ e para todo $n \geq 1$.

Demonstração. Veja-se [8, Teorema 5.1.13].

Seja $X$ um espaço topológico e seja $x_{0} \in X$ um ponto base. Então para todo $n \geq 1$ existe um homomorfismo natural

$$
h_{n}: \pi_{n}\left(X, x_{0}\right) \rightarrow H_{n}(X, \mathbb{Z})
$$

definido como segue: Se $f: I^{n} \rightarrow X$ é um representante de uma classe de homotopía $[f] \in$ $\pi_{n}\left(X, x_{0}\right)$, então $f$ induz uma aplicação $S^{n}=\frac{I^{n}}{\partial I^{n}} \rightarrow X$ e portanto uma aplicação $f_{n}: H_{n}\left(S^{n}, \mathbb{Z}\right) \rightarrow$ $H_{n}(X, \mathbb{Z})$. Dado que $H_{n}\left(S^{n}, \mathbb{Z}\right)$ é um grupo cíclico infinito (Exemplo 3.5), para cada $n \geq 1$ definimos $h_{n}(f):=f_{n}\left(\left[S^{n}\right]\right)$, onde $\left[S^{n}\right]$ é o gerador do grupo $H_{n}\left(S^{n}, \mathbb{Z}\right)$. Os homomorfismos $h_{n}$ são chamados homomorfismos de Hurewicz. Pode-se provar que $h_{n}$ de fato é um homomorfismo de grupos [2, Proposição 4.36].

TEOREMA 3.2 (The Hurewicz Theorem). Se $X$ é $n$-conexo para $n \geq 1$, então o homomorfismo de Hurewicz induz um isomorfismo $\pi_{n+1}\left(X, x_{0}\right) \rightarrow H_{n+1}(X, \mathbb{Z})$ e $H_{j}(X, \mathbb{Z})=0$ para todo $0 \leq$ $j \leq n$.

Demonstração. Veja-se [2, Teorema 4.37].

EXEMPlo 3.6. Pelo Exemplo (3.5), a esfera unitaria $S^{n}$ é $(n-1)$-conexo. Então para $n \geq 2$ temos que $\pi_{n}\left(S^{n}\right) \cong H_{n}\left(S^{n}, \mathbb{Z}\right) \cong \mathbb{Z}$.

Seja $G$ um grupo agindo sobre o espaço $X$. Dizemos que $G$ age livre e descontinua propriamente sobre $X$ se para todo $x \in X$ existe uma vizinhança $U$ de $x$ tal que $g \cdot U \cap U=\emptyset$ para todo $g \in G-\{1\}$.

TEOREMA 3.3. Seja $G$ um grupo. Então existe um $C W$-complexo contrátil $X_{G}$ tal que $G$ age livre e celularmente sobre $X_{G}$ (isto é a ação de G mapeia homeomorficamente cada célula numa outra célula, portanto $G$ age descontinua propriamente) e $X_{G} / G$ é um $C W$-complexo. Em particular, a aplicação quociente $p: X_{G} \rightarrow X_{G} / G$ é um recobrimento. 
O seguinte teorema mostra que $X_{G} / G$ é único ao menos de homotopia.

TEOREMA 3.4. Seja $G$ um grupo e sejam $X_{1}, X_{2}$ espaços topológicos Hausdorff contráteis tais que $G$ age livre e descontinua propriamente sobre $X_{1}$ e $X_{2}$. Se $X_{1} / G$ e $X_{2} / G$ são espaços paracompactos, (isto é são espaços Hausdorff tais que toda cobertura aberta admite um refinamento aberto localmente finito) então $X_{1} / G$ e $X_{2} / G$ são homotópicamente equivalentes.

Demonstração. Veja-se [8, Teorema 5.1.5].

Note que em particular os espaços $C W$-complexos são paracompactos [2, Proposição 4G.2].

DEFINIÇÃo 3.1 (Espaço Classificante). Seja $G$ um grupo. $O$ espaço $B G:=X_{G} / G$ é chamado o espaço classificante de $G$, e é único ao menos de homotopia.

Se $E G:=X_{G}$, então a aplicação quociente $p: E G \rightarrow B G$ é um recobrimento. Como $E G$ é contrátil [2, Proposição 4.1], temos que

$$
\pi_{n}\left(B G, p\left(x_{0}\right)\right)=\pi_{n}\left(E G, x_{0}\right)=0
$$

para todo $n \geq 2$. Além disso,

$$
G \cong \frac{\pi_{1}\left(B G, p\left(x_{0}\right)\right)}{p_{*}\left(\pi_{1}\left(E G, x_{0}\right)\right)}=\pi_{1}\left(B G, p\left(x_{0}\right)\right),
$$

[2, Proposição 1.40]. Assim

$$
\pi_{n}(B G)=\pi_{n}\left(B G, p\left(x_{0}\right)\right) \cong \begin{cases}G & \text { se } n=1 \\ 0 & \text { se } n \neq 1\end{cases}
$$

Reciprocamente, se $X^{\prime}$ é um $C W$-complexo conexo tal que $\pi_{1}\left(X^{\prime}, x_{0}^{\prime}\right)=G$ e para $n>1$ $\pi_{n}\left(X^{\prime}, x_{0}^{\prime}\right)=0$ em algum ponto base $x_{0}^{\prime} \in X$, então o espaço de recobrimento universal de $X^{\prime}$ tem todos seus grupos de homotopia triviais e portanto é contrátil. Assim $X^{\prime}$ é um espaço classificante de $G$.

O seguinte teorema garante que a homologia singular do espaço classificante de um grupo $G$ não difiere da homologia integral de $G$.

TEOREMA 3.5. Se $M$ é um $G$-módulo trivial, então existe um isomorfismo natural entre $H_{\bullet}(G, M)$ e $H_{\bullet}(B G, M)$. Em particular para todo $n \geq 0, H_{n}(G) \cong H_{n}(B G, \mathbb{Z})$.

Demonstração. Veja-se [8, Teorema 5.1.27].

A construção do espaço classificante dá um funtor $B(-)$ da categoria dos grupos na categoria dos espaços topológicos. Então todo homomorfismo $\alpha: G \rightarrow H$, dá-nos uma aplicação contínua $B \alpha: B G \rightarrow B H$. 


\subsection{A +-construção}

Seja $X$ um espaço topológico. Um sistema local de coeficientes sobre $X$ consiste da seguinte informação

(i) para cada $x \in X$ temos um grupo $G_{x}$ dado,

(ii) para cada $\alpha:[0,1] \rightarrow X \operatorname{com} \alpha(0)=x, \alpha(1)=y$, temos um isomorfismo $\alpha^{*}: G_{x} \rightarrow G_{y}$ que depende únicamente da classe de homotopia de $\alpha$ que une $x$ e $y$.

(iii) sejam $\alpha_{1}, \alpha_{2}:[0,1] \rightarrow X$ caminhos que unem $x \operatorname{com} y$ e $y \operatorname{com} z$, respetivamente. Se $\alpha_{1} \cdot \alpha_{2}$ é a concatenação dos caminhos $\alpha_{1}$ e $\alpha_{2}$ unindo $x \operatorname{com} z$, então

$$
\left(\alpha_{1} \cdot \alpha_{2}\right)^{*}=\alpha_{2}^{*} \circ \alpha_{1}^{*}: G_{x} \rightarrow G_{z}
$$

Se $X$ é um espaço conexo por caminhos com ponto base $x_{0} \in X$, então as condições anteriores são equivalentes a ter um grupo $G$ e uma ação de $\pi_{1}\left(X, x_{0}\right)$ sobre $G$. Em geral, para qualquer espaço $X$ e qualquer inteiro $n \geq 1$, a aplicação $x \mapsto \pi_{n}(X, x)$ é um sistema local de coeficientes sobre $X$ [10, Apêndice A.13].

Desejamos agora definir grupos de homologias $H_{\bullet}(X ; G)$ para o espaço topológico $X$ (tal que $X$ tem espaço de recobrimento universal) relativo ao sistema local de coeficientes $G$ sobre $X$ e ao ponto base $x_{0} \in X$. Desejamos que esta homologia satisfaça uma propriedade similar à Proposição (3.1), isto é se $\left\{X_{\alpha}: \alpha \in I\right\}$ são as componentes conexas por caminho de $X$, então para todo $n \geq 0$

$$
H_{n}(X ; G) \cong \bigoplus_{\alpha \in I} H_{n}\left(X_{\alpha} ;\left.G\right|_{X_{\alpha}}\right) .
$$

Assim sem perda de generalidade, podemos supor que $X$ é conexo por caminos e $p: \tilde{X} \rightarrow X$ é um espaço de recobrimento universal de $X$ com grupo fundamental $\Pi=\pi_{1}\left(X, x_{0}\right)$. Logo $X$ é o quociente de $\tilde{X}$ pela ação livre de $\Pi$ sobre $\tilde{X}$. Note que a ação de $\Pi$ sobre $\tilde{X}$ induz uma ação de $\Pi$ sobre o grupo $S_{n}(\tilde{X})$ para todo $n \geq 0$. Assim o complexo $\left(S_{\bullet}(\tilde{X}), \partial_{\bullet}\right)$ (3.1) é um complexo de cadeias de $\Pi$-módulos livres [2, Capítulo 3, Seção H]. Se $G$ é um sistema local de coeficientes sobre $X$ e $G_{0}:=G_{x_{0}}$, definimos o grupo de homologia do espaço $X$ sobre o sistema local de coeficientes $G$ como sendo

$$
H_{n}(X, A ; G):=H_{n}\left(G_{0} \otimes_{\Pi} S_{\bullet}(\tilde{X})\right)
$$

Na continuação definimos a +-construção de um espaço $X$. Isso vai permitir-nos introduzir a definição dos $K$-grupos de anéis dada por Daniel Quillen.

TEOREMA 3.6 (+-construção). Seja $X$ um espaço topológico conexo e seja $N \subseteq \pi_{1}(X)$ um subgrupo normal e perfeito de $\pi_{1}(X)$. Então existe um $C W$-complexo $X^{+}$, chamado a + -construção de $X$ relativo a $N$, tal que $X^{+}$contém $X$ e a inclusão $i: X \longrightarrow X^{+}$satisfaz as seguintes condições 
1) $\pi_{1}\left(X^{+}\right) \cong \pi_{1}(X) / N$ e o homomorfismo $i_{*}: \pi_{1}(X) \rightarrow \pi_{1}\left(X^{+}\right)$é a aplicacão quociente $\pi_{1}(X) \rightarrow \pi_{1}(X) / N$.

2) A inclusão i induz um isomorfismo $H_{n}\left(X ; i^{*}(G)\right) \rightarrow H_{n}\left(X^{+} ; G\right)$ para todo $n \geq 0$ e para quaisquer sistema local de coeficientes $G$ sobre $X^{+}$.

3) Se $f: X \rightarrow Y$ é uma aplição contínua entre espaços topológicos conexos tais que $f_{*}(N)$ é trivial em $\pi_{1}(Y)$, então existe uma aplicação contínua $f^{+}: X^{+} \rightarrow Y$, única a menos de homotopia, tal que o seguinte diagrama comuta

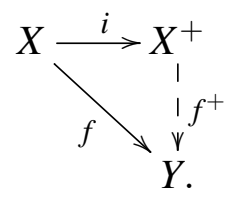

Em particular, pela segunda condição temos que

$$
H_{n}(X, \mathbb{Z}) \cong H_{n}\left(X^{+}, \mathbb{Z}\right)
$$

para todo $n \geq 0$, isto é $X$ e $X^{+}$não diferem homologicamente. Também pela terceira condição, $X^{+}$é único ao menos de homotopia.

EXEMPLO 3.7. Seja $R$ um anel com unidade e seja $X=B G L(R)$, o espaço classificante do grupo linear geral infinito $G L(R)$. Pela Proposição (2.1), o subgrupo de matrizes elementares $N=E(R)$ é um subgrupo normal e perfeito de $G L(R)=\pi_{1}(B G L(R))$. Então temos garantido a existência da +-construção do espaço $B G L(R)$ relativa a $E(R)$, denotada $B G L(R)^{+}$. Portanto para todo $n \geq 0$

$$
H_{n}\left(B G L(R)^{+}, \mathbb{Z}\right) \cong H_{n}(B G L(R), \mathbb{Z}) \cong H_{n}(G L(R)) .
$$

Além disso, se $Z$ é o espaço de recobrimento de $X$ tal que $\pi_{1}(Z)=N$, então $Z^{+}$é, a menos de homotopia, o espaço de recobrimento de $X^{+}$. Isto implica que o espaço de recobrimento de $B G L(R)^{+}$é $B E(R)^{+}$, onde estas construções são tomadas relativas ao subgrupo $E(R)$.

EXEMPLO 3.8. Considere $G=G L(R) \times G L(R)$. É fácil verificar que $B G \cong B G L(R) \times B G L(R)$ usando a caracterização dada depois da definição do espaço classificante. Por outro lado, dado que $E:=E(R) \times E(R)$ é um subgrupo normal e perfeito do grupo fundamental $\pi_{1}(B G)=G$, pelas propriedades do espaço classificante $B G L(R)^{+}$temos que a + -construção de $B G$ relativo a $E$ coincide com $B G L(R)^{+} \times B G L(R)^{+}$a menos de homotopia.

\section{3 $K$-teoria de Quillen e Milnor}

DEFINIÇão 3.2. Seja $R$ um anel com unidade. Para $n \geq 1$, o $n$-ésimo $K$-grupo do anel $R$ é o $n$-ésimo grupo de homotopia da +-construção do espaço clasificante $B G L(R)$ relativo ao grupo $E(R)$, isto é

$$
K_{n}(R):=\pi_{n}\left(B G L(R)^{+}\right)
$$


Usando a definição de $X^{+}$, se $n=1$, então

$$
K_{1}(R):=\pi_{1}\left(B G L(R)^{+}\right) \cong \frac{\pi_{1}(B G L(R))}{E(R)}=G L(R) / E(R) .
$$

Dado que $B E(R)^{+}$é um espaço de recobrimento de $B G L(R)^{+}$, então

$$
\pi_{n}\left(B G L(R)^{+}\right) \cong \pi_{n}\left(B E(R)^{+}\right)
$$

para todo $n \geq 2$. Por outro lado, dado que $B E(R)^{+}$é 1 - conexo então pelo Teorema de Hurewicz (3.2)

$$
\pi_{2}\left(B E(R)^{+}\right) \cong H_{2}\left(B E(R)^{+}\right) .
$$

Devido a (3.2) temos que homologicamente $B E(R)$ e $B E(R)^{+}$não diferem, isto é $H_{2}\left(B E(R)^{+}\right) \cong$ $H_{2}(B E(R))$. Então por (3.3) obtemos

$$
K_{2}(R):=\pi_{2}\left(B G L(R)^{+}\right) \cong H_{2}(B E(R)) \cong H_{2}(E(R)) .
$$

Portanto os novos grupos $K_{1}(R)$ e $K_{2}(R)$ coincidem com os antigos grupos $K_{1}(R)$ e $K_{2}(R)$ descritos no Capítulo 2. Se $S t(R)$ é o grupo Steinberg do anel $R$, definido no Capítulo 2, então para $n=3$ temos o seguinte resultado.

TEOREMA 3.7 (Loday). Seja $R$ um anel com unidade. Então

$$
K_{3}(R)=\pi_{3}\left(B G L(R)^{+}\right) \cong H_{3}(S t(R))
$$

Demonstração. Veja-se [3, Teorema 1.4.2].

Como $B G L(R)^{+}$é conexo por caminhos, $\pi_{0}\left(B G L(R)^{+}\right)$é trivial. Logo para incluir $K_{0}(R)$ na definição dos $K$-grupos, temos que modificar o espaço dado na Definição (3.2).

DEFINIÇão 3.3 (Quillen). Seja $R$ um anel com unidade e considere $M$ como sendo um espaço topológico discreto dado. Para $n \geq 0$, o n-ésimo $K$-grupo do anel $R$ é definido como sendo o n-ésimo grupo de homotopia do espaço $B G L(R)^{+} \times K_{0}(R)$, onde $B G L(R)^{+}$é construído relativo ao grupo $E(R)$, isto é

$$
K_{n}(R):=\pi_{n}\left(B G L(R)^{+} \times K_{0}(R)\right) .
$$

Desta maneira, $K_{0}(R):=\pi_{0}\left(B G L(R)^{+} \times M\right) \cong M$ e para $n \geq 1$ temos que $K_{n}(R):=\pi_{n}\left(B G L(R)^{+} \times\right.$ $\left.K_{0}(R)\right) \cong \pi_{n}\left(B G L(R)^{+}\right)$, recuperando assim a primeira definição.

Proposição 3.2 (Quillen, Gersten). Seja $R$ um anel com unidade. Então para $i \geq 2$ temos $K_{i}(R) \cong \pi_{i}\left(B E(R)^{+}\right)$, e para $i \geq 3, K_{i}(R) \cong \pi_{i}\left(B S t(R)^{+}\right)$. 
Note que para $n \geq 2, H_{n}\left(B E(R)^{+}\right) \cong H_{n}(E(R))$ e $H_{n}\left(B G L(R)^{+}\right) \cong H_{n}(G L(R))$, e usando o Teorema de Hurewicz, obtemos o seguinte diagrama comutativo

$$
\pi_{n}\left(B E(R)^{+}\right) \cong K_{n}(R)=\pi_{n} \underbrace{}_{H_{n}\left(E G L(R)^{+}\right)} \frac{h_{n}^{G L(R)}}{h_{n}^{E(R)}} \underset{H_{n}(G L(R))}{\longrightarrow}
$$

Para os níveis inferiores pela Proposição (2.1) e o Teorema (2.1), temos os seguintes isomorfismos

$$
K_{1}(R) \stackrel{h_{1}^{G L(R)}}{\cong} H_{1}(G L(R)), \quad K_{2}(R) \stackrel{h_{2}^{E(R)}}{\cong} H_{2}(E(R)) .
$$

Mas para o caso $n=3$, temos a seguinte proposição.

Proposição 3.3. O homomorfismo $h_{3}^{E(R)}: K_{3}(R) \rightarrow H_{3}(E(R))$ é sobrejetor.

Demonstração. O resultado segue-se ao estudar a sequência espectral

$$
E_{p, q}^{2}=H_{p}\left(E(R), H_{q}\left(K_{2}(R)\right)\right) \Rightarrow H_{p+q}(S t(R))
$$

e usando os resultados obtidos em (2.2) e (2.1), isto é $H_{1}(S t(R))=H_{2}(S t(R))=0$ e $H_{1}(E(R))=$ $\frac{E(R)}{[E(R), E(R)]}=0$.

DEFINIÇão 3.4. Seja $R$ um anel comutativo com unidade. Para $n \geq 1$, definimos o n-ésimo $K$-grupo de Milnor, denotado $K_{n}^{M}(R)$, como o grupo abeliano aditivo gerado pelos símbolos $\left\{a_{1}, \ldots, a_{n}\right\}$ com $a_{i} \in R^{\times}$para $i=1, \ldots, n$ satisfazendo as seguintes relações

i) Para todo $1 \leq i \leq n,\left\{a_{1}, \ldots, a_{i} a_{i}^{\prime}, \ldots, a_{n}\right\}=\left\{a_{1}, \ldots, a_{i}, \ldots, a_{n}\right\}+\left\{a_{1}, \ldots, a_{i}^{\prime}, \ldots, a_{n}\right\}$.

ii) Se existem $i, j(i \neq j)$ tais que $a_{i}+a_{j}=0$ ou $a_{i}+a_{j}=1$, então $\left\{a_{1}, \ldots, a_{n}\right\}=0$.

Além disso definimos $K_{0}^{M}(R):=\mathbb{Z}$.

Vejamos os níveis inferiores dos $K$-grupos de Milnor.

- Seja $n=1: K_{1}^{M}(R)$ é o grupo abeliano gerado por todos os símbolos $\{a\}$ com $a \in R^{\times}$ satisfazendo

$$
\{a b\}=\{a\}+\{b\}, \quad\{1\}=0 .
$$

Isto implica que $\left\{a^{-1}\right\}=-\{a\}$. Portanto o homomorfismo de grupos abelianos

$$
\begin{aligned}
\varphi: K_{1}^{M}(R) & \longrightarrow R^{\times} \\
\{a\} & \longmapsto a
\end{aligned}
$$

induz o isomorfismo $K_{1}^{M}(R) \cong R^{\times}$. 
- Seja $n=2: K_{2}^{M}(R)$ é o grupo abeliano gerado por todos os símbolos $\{a, b\} \operatorname{com} a, b \in R^{\times}$ tal que para todo $a, 1-a, a_{1}, a_{2}, b, b_{1}, b_{2} \in R^{\times}$,

$$
\begin{aligned}
\left\{a_{1} a_{2}, b\right\} & =\left\{a_{1}, b\right\}+\left\{a_{2}, b\right\}, \\
\left\{a, b_{1} b_{2}\right\} & =\left\{a, b_{1}\right\}+\left\{a, b_{2}\right\}, \\
\{a, 1-a\} & =0 \\
\{a,-a\} & =0 .
\end{aligned}
$$

Se $a \in R^{\times}$, é fácil ver que $\{a, 1\}=\{1, a\}=0$. Logo

$$
\left\{a^{-1}, b\right\}=-\{a, b\}, \quad\left\{a, b^{-1}\right\}=-\{a, b\} .
$$

Portanto o homomorfismo de grupos abelianos

$$
\begin{aligned}
\varphi: K_{2}^{M}(R) & \longrightarrow \frac{R^{\times} \otimes_{\mathbb{Z}} R^{\times}}{\left\langle a \otimes(1-a), b \otimes(-b): a, b, 1-a \in R^{\times}\right\rangle}, \\
\{a, b\} & \longmapsto \frac{\langle a \otimes b}{a \otimes b}
\end{aligned}
$$

é um isomorfismo.

- Seja $n=3$ : Seguindo o mesmo tratamento pode-se mostrar

$$
K_{3}^{M}(R) \cong \frac{R^{\times} \otimes_{\mathbb{Z}} R^{\times} \otimes_{\mathbb{Z}} R^{\times}}{\left\langle a_{1} \otimes a_{2} \otimes a_{3}: a_{i} \in R^{\times}, a_{i}+a_{j}=0 \text { ou } a_{i}+a_{j}=1 \text { para algum } i, j(i \neq j)\right\rangle} .
$$

LEMA 3.1. Seja $R$ um anel local com ideal maximal $\mathfrak{m}$. Se o corpo residual $R / \mathfrak{m}$ é infinito, então

$$
K_{2}^{M}(R) \cong \frac{R^{\times} \otimes_{\mathbb{Z}} R^{\times}}{\left\langle a \otimes(1-a): a, 1-a \in R^{\times}\right\rangle} .
$$

Em particular, $K_{n}^{M}(R) \cong\left(\otimes_{\mathbb{Z}}^{n} R^{\times}\right) / S$, onde $S$ é o subgrupo gerado pelos elementos $a_{1} \otimes \cdots \otimes a_{n}$ tais que existem $i, j$ com $i \neq j$ e $a_{i}+a_{j}=1$.

Demonstração. Seja $\{a, b\}^{\prime}:=\overline{a \otimes b} \in\left(R^{\times} \otimes_{\mathbb{Z}} R^{\times}\right) /\left\langle a \otimes(1-a): a, 1-a \in R^{\times}\right\rangle$. É suficiente demonstrar que $\{a,-a\}^{\prime}=0$ para todo $a \in R^{\times}$. Seja $a \in R^{\times}$tal que $1-a \in R^{\times}$. Dado que $-a=(1-a) /\left(1-a^{-1}\right)$, então

$$
\{a,-a\}^{\prime}=\left\{a,(1-a)\left(1-a^{-1}\right)^{-1}\right\}^{\prime}=\{a, 1-a\}^{\prime}-\left\{a,\left(1-a^{-1}\right)\right\}^{\prime}=\left\{a^{-1}, 1-a^{-1}\right\}^{\prime}=0 .
$$

Se $a, b \in R^{\times}$são tais que $1-a, 1-b, 1-a b \in R^{\times}$, então pelo resultado anterior

$$
\{a, b\}^{\prime}+\{b, a\}^{\prime}=\{a b,-a b\}^{\prime}-\{a,-a\}^{\prime}-\{b,-b\}^{\prime}=0 .
$$

Se $a, b \in R^{\times}$são tais que $1-a \in R^{\times}$e dado que $R / \mathfrak{m}$ é infinito, então existe $t \in R$ tal que $t, 1-t, 1-t a, 1-t b, 1-t a b \in R^{\times}$. Então pelo resultado anterior, temos

$$
\{a, b\}^{\prime}+\{b, a\}^{\prime}=\{a, t b\}^{\prime}+\{t b, a\}^{\prime}-\{a, t\}^{\prime}-\{t, a\}^{\prime}=0
$$


Se $a, b \in R^{\times}$e dado que $R / \mathfrak{m}$ é infinito, então existe $s \in R$ tal que $s, 1-s, 1-a s \in R^{\times}$. Pelo anterior, temos

$$
\{a, b\}^{\prime}+\{b, a\}^{\prime}=\{a s, b\}^{\prime}+\{b, a s\}^{\prime}-\{a, s\}^{\prime}-\{s, a\}^{\prime}=0 .
$$

Finalmente, se $a \in R^{\times}$e dado que $R / \mathfrak{m}$ é infinito, então existe $v \in R$ tal que $v, 1-v, 1-a v \in R^{\times}$. Portanto

$$
\{a,-a\}^{\prime}=\{a v,-a v\}^{\prime}-\{v,-v\}^{\prime}-\{a, v\}^{\prime}-\{v, a\}^{\prime}=0
$$

Em particular, se $R$ é um anel local com corpo residual infinito, então

$$
K_{3}^{M}(R) \cong \frac{R^{\times} \otimes_{\mathbb{Z}} R^{\times} \otimes_{\mathbb{Z}} R^{\times}}{\left\langle a_{1} \otimes a_{2} \otimes a_{3}: a_{i} \in R^{\times}, \text {existem } i, j(i \neq j) \text { tais que } a_{i}+a_{j}=1\right\rangle} .
$$

Assim, se $R$ é um anel local pelos Exemplos (2.1), (2.3) e o Teorema (2.2), os $K$-grupos de Quillen e os $K$-grupos de Milnor de $R$ coinciden para $n=0,1$ e 2;

$$
\begin{aligned}
& K_{0}^{M}(R) \cong K_{0}(R) \cong \mathbb{Z} \\
& K_{1}^{M}(R) \cong K_{1}(R) \cong R^{\times}, \\
& K_{2}^{M}(R) \cong K_{2}(R) \cong \frac{R^{\times} \otimes_{\mathbb{Z}} R^{\times}}{\left\langle a \otimes(1-a): a, 1-a \in R^{\times}\right\rangle}
\end{aligned}
$$

Definimos o homomorfismo produto entre os $K$-grupos de Milnor da seguinte forma

$$
\begin{aligned}
K_{m}^{M}(R) \otimes_{\mathbb{Z}} K_{n}^{M}(R) & \longrightarrow K_{m+n}^{M}(R) . \\
\left\{a_{1}, \ldots, a_{m}\right\} \otimes\left\{b_{1}, \ldots, b_{n}\right\} & \longmapsto\left\{a_{1}, \ldots, a_{m}\right\} \cdot\left\{b_{1}, \ldots, b_{n}\right\}:=\left\{a_{1}, \ldots, a_{m}, b_{1}, \ldots, b_{n}\right\}
\end{aligned}
$$

Como para todo $a_{1}, \ldots, a_{n} \in R^{\times}$,

$$
\left\{a_{1}, \ldots, a_{i-1}, a_{i}, a_{i+1}, a_{i+2}, \ldots, a_{n}\right\}=-\left\{a_{1}, \ldots, a_{i-1}, a_{i+1}, a_{i}, a_{i+2}, \ldots, a_{n}\right\} \in K_{n}^{M}(R),
$$

o produto definido anteriormente é anti-comutativo, isto é

$$
\left\{a_{1}, \ldots, a_{m}\right\} \cdot\left\{b_{1}, \ldots, b_{n}\right\}=(-1)^{n m}\left\{b_{1}, \ldots, b_{n}\right\} \cdot\left\{a_{1}, \ldots, a_{m}\right\} \in K_{m+n}^{M}(R)
$$

\section{4 $O$ produto nos $K$-grupos de Quillen}

Nosso objetivo nesta seção é dar um produto aos $K$-grupos de Quillen que também seja anticomutativo e estabelecer uma relação entre os $K$-grupos de Quillen e os $K$-grupos de Milnor.

Um espaço topológico $X$ com ponto base $x_{0} \in X$ é dito $H$-espaço se existe uma função contínua, chamada multiplicação, $\mu: X \times X \rightarrow X$ tal que $\mu\left(x_{0}, x_{0}\right)=x_{0}$ e as aplicações

$$
q_{1}: X \rightarrow X, \quad x \mapsto \mu\left(x, x_{0}\right), \quad q_{2}: X \rightarrow X, \quad x \mapsto \mu\left(x_{0}, x\right)
$$


são homotópicas a identidade, isto é $q_{1} \simeq_{\left\{x_{0}\right\}} i d_{X}$ e $q_{2} \simeq_{\left\{x_{0}\right\}} i d_{X}$. Além disso um $H$-espaço $X$ é dito um $H$-grupo se a multiplicação $\mu$ é associativa a menos de homotopia, isto é $\mu \circ$ $\left(\mu \times i d_{X}\right) \simeq_{\left\{\left(x_{0}, x_{0}, x_{0}\right)\right\}} \mu \circ\left(i d_{X} \times \mu\right)$ e se existe uma aplicação contínua $\eta: X \rightarrow X \operatorname{com} \eta\left(x_{0}\right)=$ $x_{0}$ tal que $\mu \circ\left(\eta, i d_{X}\right) \simeq_{\left\{x_{0}\right\}} c_{x_{0}}$ e $\mu \circ\left(i d_{X}, \eta\right) \simeq_{\left\{x_{0}\right\}} c_{x_{0}}$. onde $c_{x_{0}}(x)=x_{0}$ para todo $x \in X$.

Se $X$ é um $H$-espaço e se a aplicação $t: X \times X \rightarrow X \times X,\left(x, x^{\prime}\right) \mapsto\left(x^{\prime}, x\right)$ tem a propriedade $\mu \circ t \simeq \mu$, então dizemos que a multiplicação $\mu$ é homotopicamente comutativa. Por exemplo, se $R$ é um anel qualquer e se o homomorfismo $\mu^{\prime}: G L(R) \times G L(R) \rightarrow G L(R)$ é definido como segue

$$
\mu^{\prime}\left(\left(a_{i j}\right),\left(b_{i j}\right)\right)= \begin{cases}0 & \text { se } i \not \equiv j \quad \bmod 2 \\ a_{\frac{i+1}{2}, \frac{j+1}{2}} & \text { se } i \equiv j \equiv 1 \quad \bmod 2 \\ b_{\frac{i}{2}, \frac{j}{2}} & \text { se } i \equiv j \equiv 0 \quad \bmod 2\end{cases}
$$

tomando $\mu: B G L(R)^{+} \times B G L(R)^{+} \rightarrow B G L(R)^{+}$como sendo a aplicação contínua induzida por $B \mu^{\prime}: B G L(R) \times B G L(R) \rightarrow B G L(R)$ prova-se que $B G L(R)^{+}$é um $H$-grupo com a multiplicação $\mu$ homotópicamente comutativa [3, Teorema 1.2.6], onde $x_{0}=I_{G L(R)}$ e $I_{G L(R)}$ é a identidade de $G L(R)$.

Dados os espaços com ponto base $\left(X, x_{0}\right)$ e $\left(Y, y_{0}\right)$, definimos o espaço topológico $X \wedge Y$ como sendo o quociente $(X \times Y) / X \vee Y$, onde $X \vee Y:=\left(X \times\left\{y_{0}\right\}\right) \cup\left(\left\{x_{0}\right\} \times Y\right) \subset X \times Y$. Denotamos $x_{0} \wedge y_{0} \in X \wedge Y$ como sendo a imagem de $\left(x_{0}, y_{0}\right)$ e escolhamos $x_{0} \wedge y_{0}$ como ponto base de $X \wedge Y$.

Dadas aplicações contínuas $f: I^{n} \rightarrow X$ e $g: I^{m} \rightarrow Y$. Se $f\left(\partial I^{n}\right)=x_{0}$ e $g\left(\partial I^{m}\right)=y_{0}$, então o produto $f \times g: I^{m+n} \rightarrow X \times Y$ leva $\partial I^{m+n}=\left(\partial I^{n} \times I^{m}\right) \cup\left(I^{n} \times \partial I^{m}\right)$ para $X \vee Y$. Logo $f \times g$ induz uma aplicação contínua $f \wedge g: I^{m+n} \rightarrow X \wedge Y$ tal que $(f \wedge g)\left(\partial I^{m+n}\right)=x_{0} \wedge y_{0}$. Portanto $f \wedge g$ representa uma classe no $(m+n)$-ésimo grupo de homotopia de $X \wedge Y$. Pode-se provar que a aplicação

$$
\pi_{n}\left(X, x_{0}\right) \times \pi_{m}\left(Y, y_{0}\right) \longrightarrow \pi_{m+n}\left(X \wedge Y, x_{0} \wedge y_{0}\right), \quad([f],[g]) \mapsto[f \wedge g]
$$

está bem definida e é bilinear para todo $n, m \geq 1$ [2, Apêndice A.7]. Assim, dado um espaço $\left(X, x_{0}\right)$ e uma aplicação contínua $\left(X \wedge X, x_{0} \wedge x_{0}\right) \rightarrow\left(X, x_{0}\right)$ temos um produto bilinear induzido

$$
\star^{\prime}: \pi_{n}\left(X, x_{0}\right) \times \pi_{m}\left(X, x_{0}\right) \rightarrow \pi_{n+m}\left(X, x_{0}\right)
$$

que satisfaz

$$
x \star^{\prime} y=(-1)^{n m} y \star^{\prime} x \in \pi_{m+n}\left(X, x_{0}\right)
$$

para todo $x \in \pi_{n}\left(X, x_{0}\right)$ e para todo $y \in \pi_{m}\left(X, x_{0}\right)$. 
Sejam $R$ e $S$ dois anéis com unidade e sejam $A=\left(a_{i j}\right) \in G L_{m}(R)$ e $B \in G L_{n}(S)$. Usando o isomorfismo de $R \otimes_{\mathbb{Z}} S$-módulos $\gamma: R^{m} \otimes_{\mathbb{Z}} S^{n} \rightarrow\left(R \otimes_{\mathbb{Z}} S\right)^{m n}$ junto com o produto tensorial de matrizes, definimos

$$
A \otimes B:=\left(\begin{array}{cccc}
a_{11} B & a_{12} B & \cdots & a_{1 n} B \\
a_{21} B & a_{22} B & \cdots & a_{2 n} B \\
\vdots & \vdots & \ddots & \vdots \\
a_{n 1} B & a_{n 2} B & \cdots & a_{n n} B .
\end{array}\right) \in G L_{m n}\left(R \otimes_{\mathbb{Z}} S\right)
$$

Claramente

$$
G L_{m}(R) \times G L_{n}(S) \rightarrow G L_{m n}\left(R \otimes_{\mathbb{Z}} S\right), \quad(A, B) \mapsto A \otimes B,
$$

é um homomorfismo de grupos e induz um homomorfismo $G L(R) \times G L(S) \rightarrow G L\left(R \otimes_{\mathbb{Z}} S\right)$ que leva $E(R) \times E(S)$ para $E\left(R \otimes_{\mathbb{Z}} S\right)$. Logo temos uma aplicação contínua induzida [3, Proposição 2.1.6]

$$
\gamma^{R, S}: B G L(R)^{+} \times B G L(S)^{+} \rightarrow B G L\left(R \otimes_{\mathbb{Z}} S\right)^{+} .
$$

De acordo com [3, Proposição 2.1.8], temos uma aplicação contínua

$$
\tilde{\gamma}^{R, S}: B G L(R)^{+} \wedge B G L(S)^{+} \rightarrow B G L\left(R \otimes_{\mathbb{Z}} S\right)^{+}
$$

associativa, comutativa, única a menos de homotopia e que faz comutar o seguinte diagrama a menos de homotopia,

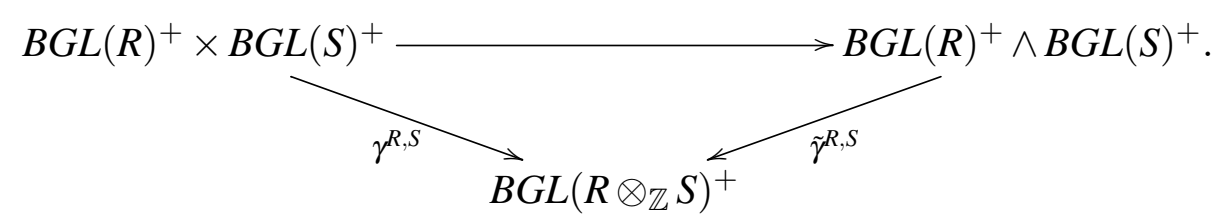

Pelo resultado anterior, para $n, m \geq 1$, a aplicação contínua $\tilde{\gamma}^{R, S}$ permite definir uma aplicação bilinear associativa [3, Teorema 2.1.11]

$$
\begin{aligned}
\star^{\prime}: \pi_{n}\left(B G L(R)^{+}\right) \times \pi_{m}\left(B G L(S)^{+}\right) & \longrightarrow \pi_{n+m}\left(B G L\left(R \otimes_{\mathbb{Z}} S\right)^{+}\right) . \\
\left([f],\left[f^{\prime}\right]\right) & \longmapsto[f] \star^{\prime}\left[f^{\prime}\right]:=\left[\tilde{\gamma}^{R, S} \circ\left(f \wedge f^{\prime}\right)\right]
\end{aligned}
$$

Se $R$ for comutativo, o homomorfismo $\alpha: R \otimes_{\mathbb{Z}} R \rightarrow R, a \otimes b \mapsto a b$ induz uma aplicação bilinear associativa dada pela composição

$$
K_{n}(R) \times K_{m}(R) \stackrel{\star^{\prime}}{\longrightarrow} K_{n+m}\left(R \otimes_{\mathbb{Z}} R\right) \stackrel{\alpha_{*}}{\longrightarrow} K_{n+m}(R),
$$

tal que para todo $x \in K_{n}(R)$ e para todo $y \in K_{m}(R)$ tem-se que $x \star y=(-1)^{n m} y \star x \in K_{n+m}(R)$, onde $\star=\alpha_{*} \circ \star^{\prime}$. Portanto temos o seguinte teorema.

TEOREMA 3.8. Se R é um anel comutativo, então temos uma multiplicação anti-comutativa associativa entre os $K$-grupos de Quillen denotada por

$$
\star: K_{n}(R) \otimes_{\mathbb{Z}} K_{m}(R) \rightarrow K_{n+m}(R) .
$$

Em particular, para todo $x \in K_{n}(R)$ e $y \in K_{m}(R)$, temos $x \star y=(-1)^{n m} y \star x$. 
Agora usando as estruturas multiplicativas dos $K$-grupos de Quillen e de Milnor, de maneira indutiva, podemos estabelecer um homomorfismo nestes grupos da seguinte maneira.

Proposição 3.4. Seja $R$ um anel local comutativo com corpo residual infinito. Então para todo $n \geq 1$ temos uma aplicação natural

$$
\tau_{n}: K_{n}^{M}(R) \rightarrow K_{n}(R)
$$

Demonstração. Para $n=1$, seja $\tau_{1}: K_{1}^{M}(R) \rightarrow K_{1}(R)$ sendo definida para todo $a \in R^{\times}$como $\tau_{1}(\{a\})=\mathbf{c}(a) \in H_{1}\left(R^{\times}\right)$. Pelo Exemplo (1.29) temos que $H_{1}\left(R^{\times}\right) \cong H_{1}(G L(R))$ (1.13) e portanto $\tau_{1}$ esta bem definida. É fácil ver que $\tau_{1}$ é um isomorfismo. O homomorfismo $\tau_{2}$ : $K_{2}^{M}(R) \rightarrow K_{2}(R)$ foi construído no Teorema de Van der Kallen-Matsumoto (2.2) e está dado por

$$
\tau_{2}(\{a, b\})=\mathbf{c}\left(\operatorname{diag}\left(a, 1, a^{-1}\right), \operatorname{diag}\left(b, b^{-1}, 1\right)\right) .
$$

Agora suponha por indução que já temos definida a aplicação $\tau_{n}: K_{n}^{M}(R) \rightarrow K_{n}(R)$. Definimos o homomorfismo $\tau_{n+1}: K_{n+1}^{M}(R) \rightarrow K_{n+1}(R)$ como sendo

$$
\tau_{n+1}\left(\left\{a_{1}, a_{2}, \ldots, a_{n+1}\right\}\right):=\tau_{1}\left(\left\{a_{1}\right\}\right) \star \tau_{n}\left(\left\{a_{2}, \ldots, a_{n+1}\right\}\right) .
$$

Pela multiplicação anti-comutativa sobre os $K$-grupos de Milnor e Quillen, é fácil verificar que esta aplicação está bem definida. Logo o seguinte diagrama comuta

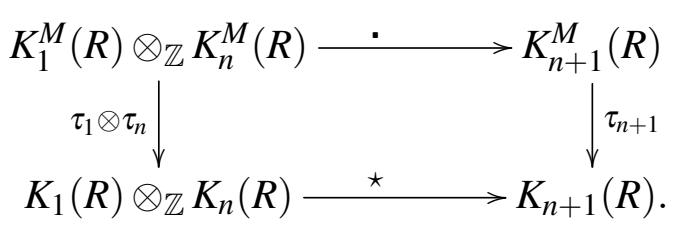

TEOREMA 3.9 (Suslin). Se R é um anel com unidade, então o núcleo do homomorfismo

$$
h_{3}^{E(R)}: K_{3}(R) \rightarrow H_{3}(E(R))
$$

coincide com a imagem do homomorfismo

$$
K_{1}(\mathbb{Z}) \otimes_{\mathbb{Z}} K_{2}(R) \rightarrow K_{3}(R) .
$$

Portanto temos a sequência exata

$$
K_{1}(\mathbb{Z}) \otimes_{\mathbb{Z}} K_{2}(R) \rightarrow K_{3}(R) \stackrel{h_{3}^{E(R)}}{\longrightarrow} H_{3}(E(R)) \rightarrow 0 .
$$

Em particular, o núcleo de $h_{3}^{E(R)}$ é 2-torção.

Demonstração. Veja-se [12, Lema 5.2]. 
Com isto descreveremos agora o homomorfismo da composição

$$
K_{n}^{M}(R) \stackrel{\tau_{n}}{\longrightarrow} K_{n}(R) \stackrel{h_{n}^{G L(R)}}{\longrightarrow} H_{n}(G L(R))
$$

quando $R$ for um anel local com corpo residual infinito.

Proposição 3.5 (Suslin). Seja $R$ um anel local com corpo residual infinito. Se $x \in K_{m}(R) e$ $y \in K_{n}(R)$, então

$$
h_{m+n}^{G L(R)}(x \star y)=\left(\psi_{m, n}\right)_{*}\left(h_{m}^{G L(R)}(x) \times h_{n}^{G L(R)}(y)\right)-m n\left(h_{m}^{G L(R)}(x) \cup h_{n}^{G L(R)}(y)\right),
$$

onde $\psi_{m, n}: G L_{m}(R) \times G L_{n}(R) \rightarrow G L_{m n}(R)$ é o produto tensorial de matrizes e o produto cup é induzido por

$$
G L_{m}(R) \times G L_{n}(R) \rightarrow G L_{m+n}(R), \quad(A, B) \longmapsto\left(\begin{array}{cc}
A & 0 \\
0 & B
\end{array}\right)
$$

Demonstração. Veja-se [11, Lema 4.2].

Corolário 3.1. Seja $R$ é um anel local com corpo residual infinito. Então o homomorfismo

$$
l_{n}:=h_{n}^{G L(R)} \circ \tau_{n}: K_{n}^{M}(R) \rightarrow H_{n}(G L(R)) \cong H_{n}\left(G L_{n}(R)\right)
$$

é dado por

$$
l_{n}\left(\left\{a_{1}, \ldots, a_{n}\right\}\right)=\left[a_{1}, \ldots, a_{n}\right]:=\boldsymbol{c}\left(A_{1, n}, \ldots, A_{n, n}\right) \in H_{n}(G L(R)),
$$

onde $A_{1, n}:=\operatorname{diag}\left(a_{1}, I_{n-1}\right) \in G L_{n}(R)$ e para $i \geq 2, A_{i, n}:=\operatorname{diag}\left(a_{i}, \ldots, a_{i}, a_{i}^{-(i-1)}, I_{n-i}\right) \in S L_{n}(R)$.

Demonstração. Usaremos indução sobre $n$. No caso $n=1$, temos $K_{1}(R) \cong K_{1}^{M}(R)=R^{\times}, \operatorname{logo}$ $l_{1}(\{a\})=\mathbf{c}(a)=[a], a \in R^{\times}$e o resultado é válido. Suponha por indução sobre $n-1$ que $l_{n-1}\left(\left\{a_{1}, \ldots, a_{n-1}\right\}\right)=\left[a_{1}, \ldots, a_{n-1}\right]$. Como $\psi_{n-1,1}: G L_{n-1}(R) \times G L_{1}(R) \rightarrow G L_{n-1}(R)$ é dado por $(A, a) \mapsto a A$, pela Proposição (3.5) temos

$$
\begin{aligned}
l_{n}\left(\left\{a_{1}, \ldots, a_{n}\right\}\right)= & h_{n}^{G L(R)}\left(\tau_{n}\left(\left\{a_{1}, \ldots a_{n}\right\}\right)\right) \\
= & h_{n}^{G L(R)}\left(\tau_{n-1}\left(\left\{a_{1}, \ldots, a_{n-1}\right\}\right) \star \tau_{1}\left(a_{n}\right)\right) \\
= & \left(\psi_{n-1,1}\right)_{*}\left(h_{n-1}\left(\tau_{n-1}\left(\left\{a_{1}, \ldots, a_{n-1}\right\}\right)\right) \times h_{1}\left(\tau_{1}\left(\left\{a_{n}\right\}\right)\right)\right) \\
& -(n-1) h_{n-1}\left(\tau_{n-1}\left(\left\{a_{1}, \ldots, a_{n-1}\right\}\right)\right) \cup h_{1}\left(\tau_{1}\left(\left\{a_{n}\right\}\right)\right) \\
= & \left(\psi_{n-1,1}\right)_{*}\left(l_{n-1}\left(\left\{a_{1}, \ldots, a_{n-1}\right\}\right) \times l_{1}\left(\left\{a_{n}\right\}\right)\right) \\
& -(n-1) l_{n-1}\left(\left\{a_{1}, \ldots, a_{n-1}\right\}\right) \cup l_{1}\left(\left\{a_{n}\right\}\right) \\
= & \left(\psi_{n-1,1}\right)_{*}\left(\mathbf{c}\left(A_{1, n-1}, \ldots, A_{n-1, n-1}\right) \times \mathbf{c}\left(a_{n}\right)\right) \\
& -(n-1) \mathbf{c}\left(A_{1, n-1}, \ldots, A_{n-1, n-1}\right) \cup \mathbf{c}\left(a_{n}\right)
\end{aligned}
$$




$$
\begin{aligned}
l_{n}\left(\left\{a_{1}, \ldots, a_{n}\right\}\right)= & \mathbf{c}\left(A_{1, n-1}, \ldots, A_{n-1, n-1}, a_{n} I_{n-1}\right) \\
& -(n-1) \mathbf{c}\left(A_{1, n}, \ldots, A_{n-1, n}, \operatorname{diag}\left(I_{n-1}, a_{n}\right)\right) \\
= & \mathbf{c}\left(A_{1, n}, \ldots, A_{n-1, n}, \operatorname{diag}\left(a_{n} I_{n-1}, 1\right)\right) \\
& +\mathbf{c}\left(A_{1, n}, \ldots, A_{n-1, n}, \operatorname{diag}\left(I_{n-1}, a_{n}^{-(n-1)}\right)\right) \\
= & \mathbf{c}\left(A_{1, n}, \ldots, A_{n-1, n}, \operatorname{diag}\left(a_{n} I_{n-1}, a_{n}^{-(n-1)}\right)\right) \\
= & \mathbf{c}\left(A_{1, n}, \ldots, A_{n, n}\right) \\
= & {\left[a_{1}, \ldots, a_{n}\right] \in H_{n}\left(G L_{n}(R)\right) \cong H_{n}(G L(R)) }
\end{aligned}
$$

OBSERVAÇ̃̃̃o 3.1. Para $i \geq 2 \operatorname{defina} A_{i, n}^{\prime}:=\operatorname{diag}\left(A_{i, n}, 1\right) \in S L_{n+1}(R)$, pelo Teorema de Estabilidade Homológica para $G L_{n}(R)$ (1.16), em $H_{n}\left(G L_{n}(R)\right)$ temos

$$
\begin{aligned}
{\left[a_{1}, \ldots, a_{n}\right]=} & \mathbf{c}\left(\operatorname{diag}\left(a_{1}, I_{n-1}, 1\right), A_{2, n}^{\prime}, \ldots, A_{n, n}^{\prime}\right) \in H_{n}\left(G L_{n+1}(R)\right) \\
= & \mathbf{c}\left(\operatorname{diag}\left(a_{1}, I_{n-1}, a_{1}^{-1}\right), A_{2, n}^{\prime}, \ldots, A_{n, n}^{\prime}\right)+\mathbf{c}\left(\operatorname{diag}\left(1, I_{n-1}, a_{1}\right), A_{2, n}^{\prime}, \ldots, A_{n, n}^{\prime}\right) \\
= & \mathbf{c}\left(\operatorname{diag}\left(a_{1}, I_{n-1}, a_{1}^{-1}\right), A_{2, n}^{\prime}, \ldots, A_{n, n}^{\prime}\right) \\
& +\mathbf{c}\left(\operatorname{diag}\left(1, I_{n-1}, a_{1}\right), \operatorname{diag}\left(a_{2}, 1, I_{n-1}\right), A_{3, n}^{\prime}, \ldots, A_{n, n}^{\prime}\right) \\
& -\mathbf{c}\left(\operatorname{diag}\left(1, I_{n-1}, a_{1}\right), \operatorname{diag}\left(1, a_{2}, I_{n-1}\right), A_{3, n}^{\prime}, \ldots, A_{n, n}^{\prime}\right) \\
= & \mathbf{c}\left(\operatorname{diag}\left(a_{1}, I_{n-1}, a_{1}^{-1}\right), A_{2, n}^{\prime}, \ldots, A_{n, n}^{\prime}\right) \\
& +\mathbf{c}\left(\operatorname{diag}\left(1, I_{n-1}, a_{1}\right), \operatorname{diag}\left(a_{2}, 1, I_{n-1}\right), A_{3, n}^{\prime}, \ldots, A_{n, n}^{\prime}\right) \\
& -\mathbf{c}\left(\operatorname{diag}\left(1, I_{n-1}, a_{1}\right), \operatorname{diag}\left(a_{2}, 1, I_{n-1}\right), A_{3, n}^{\prime}, \ldots, A_{n, n}^{\prime}\right) \\
= & \mathbf{c}\left(\operatorname{diag}\left(a_{1}, I_{n-1}, a_{1}^{-1}\right), A_{2, n}^{\prime}, \ldots, A_{n, n}^{\prime}\right) .
\end{aligned}
$$

Logo $\left[a_{1}, \ldots, a_{n}\right] \in H_{n}(S L(R))$. Em particular para $a, b, c \in R^{\times}$temos

$$
\begin{aligned}
& {[a, b]=\mathbf{c}\left(\operatorname{diag}\left(a, 1, a^{-1}\right), \operatorname{diag}\left(b, b^{-1}, 1\right)\right) \in H_{2}(S L(R)) } \\
{[a, b, c]=} & \mathbf{c}\left(\operatorname{diag}\left(a, 1,1, a^{-1}\right), \operatorname{diag}\left(b, b^{-1}, 1,1\right), \operatorname{diag}\left(c, c, c^{-2}, 1\right)\right) \\
= & \mathbf{c}\left(\operatorname{diag}\left(a, 1,1, a^{-1}, 1\right), \operatorname{diag}\left(b, b^{-1}, 1,1,1\right), \operatorname{diag}\left(c, c, c^{-2}, 1,1\right)\right) \\
= & \mathbf{c}\left(\operatorname{diag}\left(a, 1, a^{-1}, 1,1\right), \operatorname{diag}\left(b, b^{-1}, 1,1,1\right), \operatorname{diag}\left(c, c, c^{-2}, 1,1\right)\right) \\
& +\mathbf{c}\left(\operatorname{diag}\left(1,1, a, a^{-1}, 1\right), \operatorname{diag}\left(b, b^{-1}, 1,1,1\right), \operatorname{diag}\left(c, c, c^{-2}, 1,1\right)\right) \\
= & \mathbf{c}\left(\operatorname{diag}\left(a, 1, a^{-1}, 1,1\right), \operatorname{diag}\left(b, b^{-1}, 1,1,1\right), \operatorname{diag}\left(c, c, c^{-2}, 1,1\right)\right) \\
= & \mathbf{c}\left(\operatorname{diag}\left(a, 1, a^{-1}, 1,1\right), \operatorname{diag}\left(b, b^{-1}, 1,1,1\right), \operatorname{diag}\left(c, 1, c^{-1}, 1,1\right)\right) \\
& +\mathbf{c}\left(\operatorname{diag}\left(a, 1, a^{-1}, 1,1\right), \operatorname{diag}\left(b, b^{-1}, 1,1,1\right), \operatorname{diag}\left(1, c, c^{-1}, 1,1\right)\right) \\
= & \mathbf{c}\left(\operatorname{diag}\left(a, 1, a^{-1}\right), \operatorname{diag}\left(b, b^{-1}, 1\right), \operatorname{diag}\left(c, 1, c^{-1}\right)\right) \in H_{3}(S L(R))
\end{aligned}
$$

DEFINIÇÃo 3.5. Seja $R$ um anel local comutativo. Definimos o grupo

$$
K_{3}^{\text {ind }}(R):=\operatorname{coker}\left(K_{3}^{M}(R) \stackrel{\tau_{3}}{\longrightarrow} K_{3}(R)\right)
$$

chamado a parte indecomponível do terceiro $K$-grupo de $R$.

COROLÁRIO 3.2. Se Ré um anel local comutativo com corpo residual infinito, então $h_{3}^{S L(R)}$ : $K_{3}(R) \rightarrow H_{3}(S L(R))$ induz um isomorfismo $K_{3}^{\text {ind }}(R) \cong H_{3}(S L(R)) / T$, onde $T$ é o subgrupo gerado pelos elementos $[a, b, c]$ com $a, b, c \in R^{\times}$. 
Demonstração. Pelo Corolário (3.1), temos um homomorfismo bem definido

$$
\bar{h}_{3}^{S L(R)}: K_{3}^{\text {ind }}(R) \rightarrow H_{3}(S L(R)) / T, \quad x+\operatorname{Im} \tau_{3} \longmapsto h_{3}^{S L(R)}(x)+T .
$$

Dado que $h_{3}^{S L(R)}: K_{3}(R) \rightarrow H_{3}(S L(R))$ é sobrejetor, temos que $\bar{h}_{3}^{S L(R)}$ também é sobrejetor. Considere o seguinte diagrama comutativo

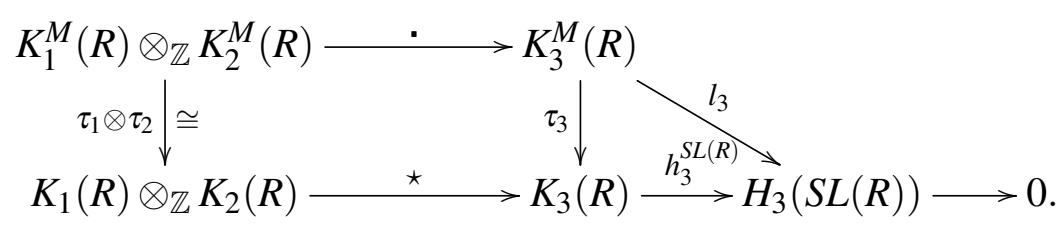

Usando a sequência exata (3.4), temos que $\operatorname{Ker} h_{3}^{S L(R)} \subseteq \operatorname{Im} l_{3}$. Agora se $x+\operatorname{Im} \tau_{3} \in \operatorname{Ker} \bar{h}_{3}^{S L(R)}$, então $h_{3}^{S L(R)}(x) \in T$ e existe $\{a, b, c\} \in K_{3}^{M}(R)$ tal que $h_{3}^{S L(R)}(x)=h_{3}^{S L(R)}\left(\tau_{3}(\{a, b, c\})\right)$, logo $x \in \operatorname{Im} \tau_{3}$. Portanto $\bar{h}_{3}^{S L(R)}$ é injetor e $K_{3}^{\text {ind }}(R) \cong H_{3}(S L(R)) / T$. 
CAPÍTULO

\section{4}

\section{$K_{3}$ DE CORPOS E O TEOREMA DE BLOCH-WIGNER}

Como resultado principal neste capítulo vamos demonstrar a existência da sequência exata de Bloch-Wigner para corpos infinitos e para isso precisaremos dos cálculos feitos no Capítulo 1 e 2 e a caracterização obtida no Corolário (3.2).

\subsection{Terceira homologia de $G L_{2}$ e o grupo de Bloch}

Seja $R$ um anel comutativo com unidade. Seja $Q(R)$ o $\mathbb{Z}$-módulo livre com base nos símbolos $[a]$, onde $a, 1-a \in R^{\times}$e seja $\left(R^{\times} \otimes_{\mathbb{Z}} R^{\times}\right)_{\sigma}$ o quociente de $R^{\times} \otimes_{\mathbb{Z}} R^{\times}$pelo subgrupo gerado por elementos da forma $a \otimes b+b \otimes a$, com $a, b \in R^{\times}$. Considere o homomorfismo

$$
\lambda^{\prime}: Q(R) \longrightarrow R^{\times} \otimes_{\mathbb{Z}} R^{\times}, \quad[a] \longmapsto a \otimes(1-a) .
$$

Seja $\mathfrak{p}(R)$ o pré-grupo de Bloch de $R$, isto é o quociente de $Q(R)$ pelo submódulo gerado pelos elementos da forma $[a]-[b]+\left[\frac{b}{a}\right]-\left[\frac{1-a^{-1}}{1-b^{-1}}\right]+\left[\frac{1-a}{1-b}\right]$. Por cálculos diretos podemos ver que

$$
\lambda^{\prime}\left([a]-[b]+\left[\frac{b}{a}\right]-\left[\frac{1-a^{-1}}{1-b^{-1}}\right]+\left[\frac{1-a}{1-b}\right]\right)=a \otimes\left(\frac{1-a}{1-b}\right)+\left(\frac{1-a}{1-b}\right) \otimes a .
$$

Então temos um homomorfismo induzido bem definido

$$
\lambda: \mathfrak{p}(R) \longrightarrow\left(R^{\times} \otimes_{\mathbb{Z}} R^{\times}\right)_{\sigma}, \quad[a] \longmapsto a \otimes(1-a) .
$$

Note que o elemento representado por $a \otimes b$ em $\left(R^{\times} \otimes_{\mathbb{Z}} R^{\times}\right)_{\sigma}$ será denotado novamente por $a \otimes b$.

DEFINIÇão 4.1. Definimos o grupo de Bloch de um anel comutativo $R$, denotado por $B(R)$, como sendo

$$
B(R):=\operatorname{Ker}\left(\mathfrak{p}(R) \stackrel{\lambda}{\longrightarrow}\left(R^{\times} \otimes_{\mathbb{Z}} R^{\times}\right)_{\sigma}\right) .
$$


Se $R$ é um anel local com corpo residual infinito, então pelo Teorema de Van der KallenMatsumoto (2.2) e o Lema (3.1) temos

$$
K_{2}(R) \cong K_{2}^{M}(R)=\frac{R^{\times} \otimes_{\mathbb{Z}} R^{\times}}{\left\langle a \otimes(1-a): a, 1-a \in R^{\times}\right\rangle} .
$$

Observe que

$$
\operatorname{coker}(\lambda)=\frac{R^{\times} \otimes_{\mathbb{Z}} R^{\times}}{\left\langle a \otimes(1-a), b \otimes c+c \otimes b: a, 1-a, b, c \in R^{\times}\right\rangle} \cong K_{2}^{M}(R) .
$$

Portanto temos a sequência exata

$$
0 \longrightarrow B(R) \longrightarrow \mathfrak{p}(R) \stackrel{\lambda}{\longrightarrow}\left(R^{\times} \otimes_{\mathbb{Z}} R^{\times}\right)_{\sigma} \longrightarrow K_{2}(R) \longrightarrow 0 .
$$

Neste capítulo sempre assumiremos que $F$ é um corpo infinito e usaremos os resultados dos capítulos anteriores em forma particular para $F$. Agora consideremos a sequência espectral (2.3) que introduzimos e estudamos no Capítulo 2.

$$
E_{p, q}^{1}=H_{q}\left(G L_{2}(F), C_{p}\left(F^{2}\right)\right) \Longrightarrow H_{p+q}\left(G L_{2}(F)\right)
$$

Esta sequência espectral induz uma filtração de $H_{3}\left(G L_{2}(F)\right)$

$$
0 \subseteq F_{0} H_{3}\left(G L_{2}(F)\right) \subseteq F_{1} H_{3}\left(G L_{2}(F)\right) \subseteq F_{2} H_{3}\left(G L_{2}(F)\right) \subseteq H_{3}\left(G L_{2}(F)\right)
$$

tal que

$$
\begin{aligned}
& E_{0,3}^{\infty} \cong F_{0} H_{3}\left(G L_{2}(F)\right), \\
& E_{1,2}^{\infty} \cong \frac{F_{1} H_{3}\left(G L_{2}(F)\right)}{F_{0} H_{3}\left(G L_{2}(F)\right)}, \\
& E_{2,1}^{\infty} \cong \frac{F_{2} H_{3}\left(G L_{2}(F)\right)}{F_{1} H_{3}\left(G L_{2}(F)\right)}, \\
& E_{3,0}^{\infty} \cong \frac{H_{3}\left(G L_{2}(F)\right)}{F_{2} H_{3}\left(G L_{2}(F)\right)} .
\end{aligned}
$$

Note que pelo Lema (2.8), $E_{3,0}^{\infty}=\operatorname{Ker} d_{3,0}^{3}=B(F)$. Assim obtemos a seguinte sequência exata

$$
0 \longrightarrow F_{2} H_{3}\left(G L_{2}(F)\right) \longrightarrow H_{3}\left(G L_{2}(F)\right) \longrightarrow B(F) \longrightarrow 0 \text {. }
$$

Dado que $E_{2,1}^{3}=0$ temos que $F_{1} H_{3}\left(G L_{2}(F)\right)=F_{2} H_{3}\left(G L_{2}(F)\right)$. Além disso temos o homomorfismo sobrejetor

$$
H_{3}\left(T_{2}\right) \rightarrow E_{0,3}^{\infty} \cong F_{0} H_{3}\left(G L_{2}(F)\right) \subseteq H_{3}\left(G L_{2}(F)\right),
$$

que implica

$$
F_{0} H_{3}\left(G L_{2}(F)\right)=\operatorname{Im}\left(H_{3}\left(T_{2}\right) \longrightarrow H_{3}\left(G L_{2}(F)\right)\right),
$$

Desejamos estudar o homomorfismo

$$
H_{2}\left(T_{2}\right)^{\sigma} \rightarrow E_{1,2}^{2}=\left(F^{\times} \otimes_{\mathbb{Z}} F^{\times}\right)^{\sigma} \rightarrow E_{1,2}^{\infty}=\frac{F_{1} H_{3}\left(G L_{2}(F)\right)}{F_{0} H_{3}\left(G L_{2}(F)\right)} \subseteq \frac{H_{3}\left(G L_{2}(F)\right)}{H_{3}\left(T_{2}\right)} .
$$


Seja $G$ um grupo, $g \in G$ e seja $M$ um $G$-módulo. Considere o automomorfismo de complexos de cadeias

$$
\begin{aligned}
\tau_{g}: B_{\bullet}(G) \otimes_{G} M & \longrightarrow B_{\bullet}(G) \otimes_{G} M, \\
{\left[g_{1}\left|g_{2}\right| \cdots \mid g_{n}\right] \otimes m } & \longmapsto\left[g g_{1} g^{-1}\left|g g_{2} g^{-1}\right| \cdots \mid g g_{n} g^{-1}\right] \otimes g m
\end{aligned}
$$

e para cada $n \geq 0$ considere o homomorfismo

$$
\begin{aligned}
\varphi_{g}: B_{n}(G) \otimes_{G} M & \longrightarrow B_{n+1}(G) \otimes_{G} M \\
{\left[g_{1}\left|g_{2}\right| \cdots \mid g_{n}\right] \otimes m } & \longmapsto \sum_{j=0}^{n}(-1)^{j}\left[g_{1}|\cdots| g_{j}\left|g^{-1}\right| g g_{j+1} g^{-1}|\cdots| g g_{n} g^{-1}\right] \otimes m
\end{aligned}
$$

Note que se $n=0$, então

$$
\left(d_{1} \otimes i d\right) \circ \varphi_{g}([] \otimes m)=\left(g^{-1}[]-[]\right) \otimes m=[] \otimes g m-[] \otimes m=\left(\tau_{g}-i d\right)([] \otimes m),
$$

e se $n \geq 1$, então

$$
\begin{aligned}
\left(\left(d_{n+1} \otimes i d\right) \circ \varphi_{g}\right)\left(\left[g_{1}|\cdots| g_{n}\right] \otimes m\right)= & \left(d_{n+1} \otimes i d\right)\left(\left[g^{-1}\left|g g_{1} g^{-1}\right| \cdots \mid g g_{n} g^{-1}\right] \otimes m\right. \\
& -\left[g_{1}\left|g^{-1}\right| g g_{2} g^{-1}|\cdots| g g_{n} g^{-1}\right] \otimes m+\cdots \\
& \left.+(-1)^{n}\left[g_{1}\left|g_{2}\right| \cdots\left|g_{n}\right| g^{-1}\right] \otimes m\right) \\
= & g^{-1}\left[g g_{1} g^{-1}|\cdots| g g_{n} g^{-1}\right] \otimes m \\
& -\left[g_{1}|\cdots| g_{n}\right] \otimes m-\varphi_{g}\left(g_{1}\left[g_{2}|\cdots| g_{n}\right] \otimes m\right. \\
& -\left[g_{1} g_{2}|\cdots| g_{n}\right] \otimes m+\left[g_{1}\left|g_{2} g_{3}\right| \cdots \mid g_{n}\right] \otimes m+\cdots+ \\
& +(-1)^{n-1}\left[g_{1}\left|g_{2}\right| \cdots \mid g_{n-1} g_{n}\right] \otimes m \\
& \left.+(-1)^{n}\left[g_{1}|\cdots| g_{n-1}\right] \otimes m\right) \\
= & {\left[g g_{1} g^{-1}|\cdots| g g_{n} g^{-1}\right] \otimes g m-\left[g_{1}|\cdots| g_{n}\right] \otimes m } \\
& -\left(\varphi_{g} \circ\left(d_{n} \otimes i d\right)\right)\left(\left[g_{1}|\cdots| g_{n}\right] \otimes m\right) .
\end{aligned}
$$

Logo

$$
\left(\left(d_{n+1} \otimes i d\right) \circ \varphi_{g}+\varphi_{g} \circ\left(d_{n} \otimes i d\right)\right)\left(\left[g_{1}|\cdots| g_{n}\right] \otimes m\right)=\left(\tau_{g}-i d\right)\left(\left[g_{1} \mid \cdots g_{n}\right] \otimes m\right)
$$

Portanto, $\tau_{g}$ é homotópica ao morfismo de complexos identidade.

LEMA 4.1. Seja $u \in H_{2}\left(T_{2}\right)^{\sigma}$ e seja $h \in B_{2}\left(G L_{2}(F)\right)_{T_{2}}$ um ciclo representante para $u$. Considere $\omega=\left(\begin{array}{ll}0 & 1 \\ 1 & 0\end{array}\right) \in G L_{2}(F)$ e seja $b \in B_{3}\left(G L_{2}(F)\right)_{T_{2}}$ tal que $\tau_{\omega}(h)-h=d_{3}(b)$. Então a imagem de u pelo homomorfismo

$$
H_{2}\left(T_{2}\right)^{\sigma} \longrightarrow \frac{F_{2} H_{3}\left(G L_{2}(F)\right)}{H_{3}\left(T_{2}\right)} \subseteq \frac{H_{3}\left(G L_{2}(F)\right)}{H_{3}\left(T_{2}\right)}
$$

coincide com a classe do ciclo $b-\varphi_{\omega}(h)$.

Demonstração. Seja $B_{\bullet}\left(G L_{2}(F)\right) \stackrel{\varepsilon}{\longrightarrow} \mathbb{Z}$ a resolução barra de $\mathbb{Z}$ sobre $G L_{2}(F)$. Então a sequência espectral (2.3) coincide com a sequência espectral induzida pelo complexo duplo dado por $B_{\bullet}\left(G L_{2}(F)\right) \otimes_{G L_{2}(F)} C_{\bullet}\left(F^{2}\right)$. Note que o morfismo de augmentação $B_{\bullet}\left(G L_{2}(F)\right) \stackrel{\varepsilon}{\longrightarrow} \mathbb{Z}$ 
induz uma equivalência fraca $B_{\bullet}\left(G L_{2}(F)\right) \otimes_{G L_{2}(F)} C_{\bullet}\left(F^{2}\right) \longrightarrow B_{\bullet}\left(G L_{2}(F)\right)_{G L_{2}(F)}$. Considere o seguinte diagrama

$$
\begin{aligned}
& B_{3}\left(G L_{2}(F)\right)_{G L_{2}(F)} \stackrel{\varepsilon}{\longleftarrow} B_{3}\left(G L_{2}(F)\right) \otimes_{G L_{2}(F)} C_{0}\left(F^{2}\right) \\
& d_{3} \otimes i d \downarrow \\
& B_{2}\left(G L_{2}(F)\right) \otimes_{G L_{2}(F)} C_{0}\left(F^{2}\right) \stackrel{i d \otimes \partial_{1}}{\longleftarrow} B_{2}\left(G L_{2}(F)\right) \otimes_{G L_{2}(F)} C_{1}\left(F^{2}\right) .
\end{aligned}
$$

Se $h=\left[g_{1} \mid g_{2}\right]-\left[g_{2} \mid g_{1}\right]$ para algum par $g_{1}, g_{2} \in G L_{2}(F)$, então $h \otimes\left(\left\langle e_{1}\right\rangle,\left\langle e_{2}\right\rangle\right)$ representa $u$ em $B_{2}\left(G L_{2}(F)\right) \otimes_{G L_{2}(F)} C_{1}\left(F^{2}\right)$. Temos

$$
\begin{aligned}
\varphi_{\omega}(h)= & \varphi_{\omega}\left[g_{1} \mid g_{2}\right]-\varphi_{\omega}\left[g_{2} \mid g_{1}\right] \\
= & {\left[\omega^{-1}\left|\omega g_{1} \omega^{-1}\right| \omega g_{2} \omega^{-1}\right]-\left[g_{1}\left|\omega^{-1}\right| \omega g_{2} \omega^{-1}\right]+\left[g_{1}\left|g_{2}\right| \omega^{-1}\right] } \\
& -\left[\omega^{-1}\left|\omega g_{2} \omega^{-1}\right| \omega g_{1} \omega^{-1}\right]+\left[g_{2}\left|\omega^{-1}\right| \omega g_{1} \omega^{-1}\right]-\left[g_{2}\left|g_{1}\right| \omega^{-1}\right]
\end{aligned}
$$

$\mathrm{e}$

$$
\begin{aligned}
d_{3}\left(\varphi_{\omega}(h)\right)= & d_{3}\left(\left[\omega^{-1}\left|\omega g_{1} \omega^{-1}\right| \omega g_{2} \omega^{-1}\right]-\left[g_{1}\left|\omega^{-1}\right| \omega g_{2} \omega^{-1}\right]+\left[g_{1}\left|g_{2}\right| \omega^{-1}\right]\right) \\
& -d_{3}\left(\left[\omega^{-1}\left|\omega g_{2} \omega^{-1}\right| \omega g_{1} \omega^{-1}\right]-\left[g_{2}\left|\omega^{-1}\right| \omega g_{1} \omega^{-1}\right]+\left[g_{2}\left|g_{1}\right| \omega^{-1}\right]\right) \\
= & \omega^{-1} \tau_{\omega}(h)-h .
\end{aligned}
$$

Assim

$$
\left(d_{3} \otimes i d\right)\left(b \otimes\left(\left\langle e_{2}\right\rangle\right)-\varphi_{\omega}(h) \otimes\left(\left\langle e_{1}\right\rangle\right)\right)=h \otimes\left(\left\langle e_{2}\right\rangle\right)-h \otimes\left(\left\langle e_{1}\right\rangle\right)=\left(i d \otimes \partial_{1}\right)\left(h \otimes\left(\left\langle e_{1}\right\rangle,\left\langle e_{2}\right\rangle\right)\right) .
$$

Note que

$$
\varepsilon\left(b \otimes\left(\left\langle e_{2}\right\rangle\right)-\varphi_{\omega}(h) \otimes\left(\left\langle e_{1}\right\rangle\right)\right)=b-\varphi_{\omega}(h)
$$

representa a imagem de $u$ em $B_{3}\left(G L_{2}(F)\right)_{G L_{2}(F)}$. Então a classe do ciclo $b-\varphi_{\omega}(h)$ representa a imagem de $u$ em $H_{3}\left(G L_{2}(F)\right)=H_{3}\left(B_{\bullet}\left(G L_{2}(F)\right)_{G L_{2}(F)}\right)$.

\subsection{Terceira homologia de $G M_{2}$}

Seja $G M_{2}(F)$ o subgrupo das matrizes monomiais de $G L_{2}(F)$, isto é

$$
G M_{2}(F):=\left\{\left(\begin{array}{ll}
a & 0 \\
0 & b
\end{array}\right),\left(\begin{array}{cc}
0 & b^{\prime} \\
a^{\prime} & 0
\end{array}\right): a, b, a^{\prime}, b^{\prime} \in F^{\times}\right\} \subseteq G L_{2}(F) .
$$

Considerando $\Sigma_{2} \cong\left\{\left(\begin{array}{ll}1 & 0 \\ 0 & 1\end{array}\right),\left(\begin{array}{ll}0 & 1 \\ 1 & 0\end{array}\right)\right\}$, então $G M_{2}(F) \cong T_{2} \rtimes \Sigma_{2}$. A sequência exata

$$
1 \longrightarrow T_{2} \longrightarrow G M_{2}(F) \longrightarrow \Sigma_{2} \longrightarrow 1
$$

dá-nos a sequência espectral de Lyndon/Hochschild-Serre

$$
\mathscr{E}_{p, q}^{2}=H_{p}\left(\Sigma_{2}, H_{q}\left(T_{2}\right)\right) \Longrightarrow H_{p+q}\left(G M_{2}(F)\right)
$$


Calculamos algumos termos desta sequência espectral. Pelo Exemplo (1.11),

$$
\mathscr{E}_{p, 0}^{2}=H_{p}\left(\Sigma_{2}, H_{0}\left(T_{2}\right)\right)=H_{p}\left(\Sigma_{2}\right) \cong \begin{cases}\mathbb{Z} & \text { se } p=0 \\ \mathbb{Z} / 2 \mathbb{Z} & \text { se } p \text { é impar } \\ 0 & \text { se } p \text { é par. }\end{cases}
$$

e pelo Exemplo (1.15),

$$
\mathscr{E}_{p, 1}^{2}=H_{p}\left(\Sigma_{2}, H_{1}\left(T_{2}\right)\right)=H_{p}\left(\Sigma_{2}, F^{\times} \oplus F^{\times}\right) \cong \begin{cases}F^{\times}, & p=0, \\ 0, & p \neq 0 .\end{cases}
$$

Pelo isomorfismo (1.8) e pelos Exemplos (1.7) e (1.15), temos

$$
\begin{aligned}
\mathscr{E}_{p, 2}^{2}= & H_{p}\left(\Sigma_{2}, H_{2}\left(T_{2}\right)\right)=H_{p}\left(\Sigma_{2}, H_{2}\left(F^{\times}\right) \oplus H_{2}\left(F^{\times}\right) \oplus\left(F^{\times} \otimes_{\mathbb{Z}} F^{\times}\right)\right) \\
& =H_{p}\left(\Sigma_{2}, H_{2}\left(F^{\times}\right) \oplus H_{2}\left(F^{\times}\right)\right) \oplus H_{p}\left(\Sigma_{2}, F^{\times} \otimes_{\mathbb{Z}} F^{\times}\right) \\
& =H_{p}\left(\Sigma_{2}, F^{\times} \otimes_{\mathbb{Z}} F^{\times}\right)= \begin{cases}\left(F^{\times} \otimes_{\mathbb{Z}} F^{\times}\right)_{\Sigma_{2}} & \text { se } p=0, \\
H_{p}\left(\Sigma_{2}, \mu_{2^{\infty}}(F) \otimes_{\mathbb{Z}} \mu_{2^{\infty}}(F)\right) & \text { se } p \text { é impar, } \\
\left\langle\overline{r \otimes r} \in\left(F^{\times} \otimes_{\mathbb{Z}} F^{\times}\right)_{\Sigma_{2}}: r \in F^{\times}\right\rangle & \text {se } p \text { é par. }\end{cases}
\end{aligned}
$$

Note que na notação das definições (2.5)

$$
\begin{gathered}
\left(F^{\times} \otimes_{\mathbb{Z}} F^{\times}\right)_{\sigma}=\frac{F^{\times} \otimes_{\mathbb{Z}} F^{\times}}{\left\langle a \otimes b-\sigma(a \otimes b): a, b \in F^{\times}\right\rangle}=\left(F^{\times} \otimes_{\mathbb{Z}} F^{\times}\right)_{\Sigma_{2}}, \\
\left(F^{\times} \otimes_{\mathbb{Z}} F^{\times}\right)^{\sigma}=\{a \otimes b: \sigma(a \otimes b)=a \otimes b\}=\left(F^{\times} \otimes_{\mathbb{Z}} F^{\times}\right)^{\Sigma_{2}} .
\end{gathered}
$$

Denotaremos $\mathscr{E}_{0,3}^{2}=H_{3}\left(T_{2}\right)_{\Sigma_{2}}$ como $H_{3}\left(T_{2}\right)_{\sigma}$. Então a segunda folha da sequência espectral $\mathscr{E}_{p, q}^{2}$ é dada por

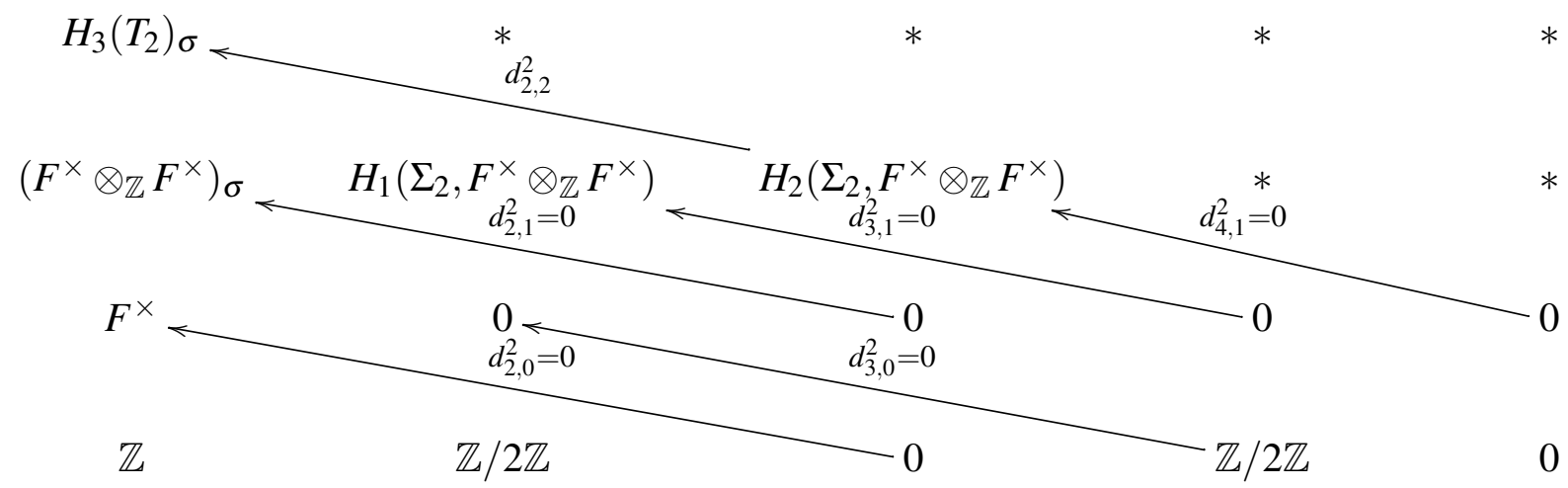

LEMA 4.2. Na sequência espectral anterior, o diferencial $d_{2,2}^{2}: H_{2}\left(\Sigma_{2}, F^{\times} \otimes_{\mathbb{Z}} F^{\times}\right) \rightarrow H_{3}\left(T_{2}\right)_{\sigma}$ é trivial. 
Demonstração. A sequência espectral coincide com a sequência induzida pelo complexo duplo $B \bullet\left(\Sigma_{2}\right) \otimes_{\Sigma_{2}} B \bullet\left(G M_{2}(F)\right)_{T_{2}}$. Considere o seguinte diagrama

$$
\begin{aligned}
B_{3}\left(G M_{2}(F)\right)_{T_{2}} \longleftarrow & B_{1}\left(\Sigma_{2}\right) \otimes \Sigma_{2} B_{3}\left(G M_{2}(F)\right)_{T_{2}} \\
i d \otimes d_{3} & \downarrow \\
& B_{1}\left(\Sigma_{2}\right) \otimes \Sigma_{2} B_{2}\left(G M_{2}(F)\right)_{T_{2}} \stackrel{d_{2} \otimes i d}{=} B_{2}\left(\Sigma_{2}\right) \otimes_{\Sigma_{2}} B_{2}\left(G M_{2}(F)\right)_{T_{2}} .
\end{aligned}
$$

Pelo Exemplo (1.15), $H_{2}\left(\Sigma_{2}, F^{\times} \otimes_{\mathbb{Z}} F^{\times}\right)=\left\langle\overline{a \otimes a} \in\left(F^{\times} \otimes_{\mathbb{Z}} F^{\times}\right)_{\Sigma_{2}}: r \in F^{\times}\right\rangle$. Sabemos que usando o homomorfismo $F^{\times} \otimes_{\mathbb{Z}} F^{\times} \stackrel{\cup}{\longrightarrow} H_{2}\left(T_{2}\right)$, temos $a \otimes a \longmapsto a \cup a:=c(\operatorname{diag}(a, 1), \operatorname{diag}(1, a))$. Se $\omega=\left(\begin{array}{ll}0 & 1 \\ 1 & 0\end{array}\right) \in \Sigma_{2}$, então

$$
\left(i d \otimes d_{3}\right)\left(-[\omega] \otimes \varphi_{\omega}^{\prime}(a \cup a)\right)=\omega[\omega] \otimes(a \cup a)+[\omega] \otimes(a \cup a)=\left(d_{2} \otimes i d\right)([\omega] \otimes(a \cup a)),
$$

onde $\varphi_{\omega}^{\prime}: B_{2}\left(G M_{2}(F)\right)_{T_{2}} \longrightarrow B_{3}\left(G M_{2}(F)\right)_{T_{2}}$ é definido de igual maneira que o homomorfismo (4.6). Agora

$$
\begin{aligned}
\left(d_{1} \otimes i d\right)\left(-[\omega] \otimes \varphi_{\omega}^{\prime}(a \cup a)\right) & =[] \otimes \varphi_{\omega}^{\prime}(a \cup a)-\omega[] \otimes \varphi_{\omega}^{\prime}(a \cup a) \in B_{0}\left(\Sigma_{2}\right) \otimes_{\Sigma_{2}} B_{3}\left(G M_{2}(F)\right)_{T_{2}} \\
& =\varphi_{\omega}^{\prime}(a \cup a)-\omega \cdot \varphi_{\omega}^{\prime}(a \cup a) \in B_{3}\left(G M_{2}(F)\right)_{T_{2}} .
\end{aligned}
$$

Assim, $d_{2,2}^{2}(\overline{a \otimes a})=\overline{\varphi_{\omega}^{\prime}(a \cup a)-\omega \cdot \varphi_{\omega}^{\prime}(a \cup a)}=\overline{0} \in H_{3}\left(T_{2}\right)_{\sigma}$.

Portanto, a terceira folha da sequência espectral $\mathscr{E}_{p, q}^{2}$ é dada por

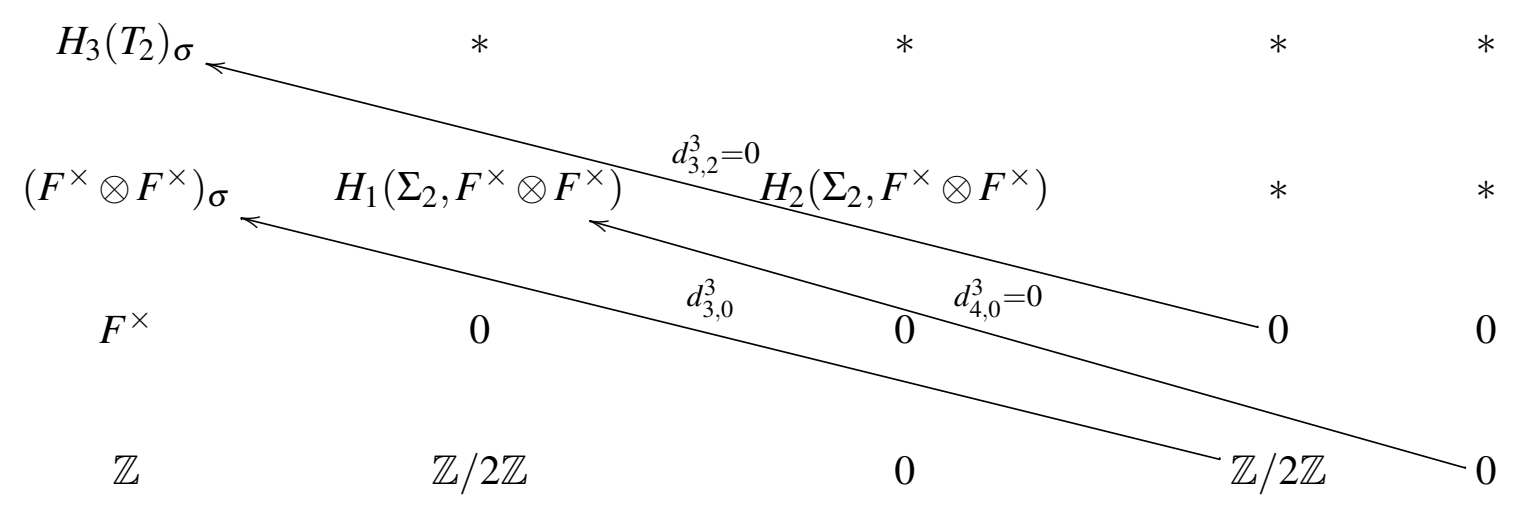

Já que o homomorfismo $G M_{2}(F) \rightarrow \Sigma_{2}$ cinde com a inclusão $\Sigma_{2} \stackrel{i}{\rightarrow} G M_{2}(F)$, então pelo Exemplo (1.28) temos que $d_{3,0}^{3}=0$. A sequência espectral $\mathscr{E}_{p, q}^{2}$ induz uma filtração de $H_{3}\left(G M_{2}(F)\right)$

$0=F_{-1}^{\prime} H_{3}\left(G M_{2}(F)\right) \subseteq F_{0}^{\prime} H_{3}\left(G M_{2}(F)\right) \subseteq F_{1}^{\prime} H_{3}\left(G M_{2}(F)\right) \subseteq F_{2}^{\prime} H_{3}\left(G M_{2}(F)\right) \subseteq H_{3}\left(G M_{2}(F)\right)$, tal que

$$
\begin{aligned}
& \mathscr{E}_{0,3}^{\infty}=F_{0}^{\prime} H_{3}\left(G M_{2}(F)\right), \\
& \mathscr{E}_{1,2}^{\infty}=\frac{F_{1}^{\prime} H_{3}\left(G M_{2}(F)\right)}{F_{0}^{\prime} H_{3}\left(G M_{2}(F)\right)}, \\
& \mathscr{E}_{2,1}^{\infty}=\frac{F_{2}^{\prime} H_{3}\left(G M_{2}(F)\right)}{F_{1}^{\prime} H_{3}\left(G M_{2}(F)\right)}, \\
& \mathscr{E}_{3,0}^{\infty}=\frac{H_{3}\left(G M_{2}(F)\right)}{F_{2}^{\prime} H_{3}\left(G M_{2}(F)\right)} .
\end{aligned}
$$


Agora é fácil verificar que

$$
\begin{aligned}
F_{2}^{\prime} H_{3}\left(G M_{2}(F)\right) & =F_{1}^{\prime} H_{3}\left(G M_{2}(F)\right), \\
H_{3}\left(T_{2}\right)_{\sigma} & =F_{0}^{\prime} H_{3}\left(G M_{2}(F)\right), \\
H_{1}\left(\Sigma_{2}, F^{\times} \otimes F^{\times}\right) & =\frac{F_{2}^{\prime} H_{3}\left(G M_{2}(F)\right)}{H_{3}\left(T_{2}\right)_{\Sigma_{2}}}, \\
H_{3}\left(\Sigma_{2}\right)=\mathbb{Z} / 2= & \frac{H_{3}\left(G M_{2}(F)\right)}{F_{2}^{\prime} H_{3}\left(G M_{2}(F)\right)} .
\end{aligned}
$$

Assim temos as seguintes sequência exatas

$$
\begin{gathered}
0 \longrightarrow H_{3}\left(T_{2}\right)_{\sigma} \longrightarrow F_{2}^{\prime} H_{3}\left(G M_{2}(F)\right) \longrightarrow H_{1}\left(\Sigma_{2}, F^{\times} \otimes F^{\times}\right) \longrightarrow 0, \\
0 \longrightarrow F_{2}^{\prime} H_{3}\left(G M_{2}(F)\right) \longrightarrow H_{3}\left(G M_{2}(F)\right) \longrightarrow H_{3}\left(\Sigma_{2}\right) \longrightarrow 0 .
\end{gathered}
$$

Como (4.9) cinde pela inclusão $\Sigma_{2} \stackrel{i}{\rightarrow} G M_{2}(F)$, obtemos a decomposição

$$
H_{3}\left(G M_{2}(F)\right) \cong H_{3}\left(\Sigma_{2}\right) \oplus F_{2}^{\prime} H_{3}\left(G M_{2}(F)\right) .
$$

Dado que $F_{0}^{\prime} H_{3}\left(G M_{2}(F)\right)=\operatorname{Im}\left(H_{3}\left(T_{2}\right) \longrightarrow H_{3}\left(G M_{2}(F)\right)\right)$, então pela igualdade (4.4) e pela comutatividade do seguinte diagrama

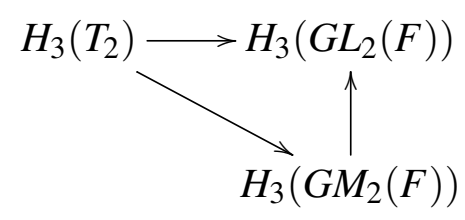

temos um homomorfismo sobrejetor

$$
H_{3}\left(T_{2}\right)_{\sigma}=F_{0}^{\prime} H_{3}\left(G M_{2}(F)\right) \longrightarrow F_{0} H_{3}\left(G L_{2}(F)\right) .
$$

Agora podemos estudar o homomorfismo

$$
H_{2}\left(T_{2}\right)^{\sigma} \rightarrow \mathscr{E}_{1,2}^{2}=H_{1}\left(\Sigma_{2}, H_{2}\left(T_{2}\right)\right) \rightarrow \mathscr{E}_{1,2}^{\infty}=\frac{F_{2}^{\prime} H_{3}\left(G M_{2}(F)\right)}{F_{0}^{\prime} H_{3}\left(G M_{2}(F)\right)} \subseteq \frac{H_{3}\left(G M_{2}(F)\right)}{H_{3}\left(T_{2}\right)}
$$

de forma similar ao Lema (4.1).

LEMA 4.3. Seja $u \in H_{2}\left(T_{2}\right)^{\sigma}$ e seja $h \in B_{2}\left(T_{2}\right)_{T_{2}}$ um ciclo representante para $u$. Considere em $G M_{2}(F)$ a matriz $\omega=\left(\begin{array}{ll}0 & 1 \\ 1 & 0\end{array}\right)$ e seja $b \in B_{3}\left(T_{2}\right)_{T_{2}}$ tal que $\tau_{\omega}^{\prime}(h)-h=d_{3}(b)$, onde

$$
\tau_{\omega}^{\prime}: B_{2}\left(G M_{2}(F)\right)_{T_{2}} \rightarrow B_{2}\left(G M_{2}(F)\right)_{T_{2}}
$$

é o automorfismo induzido por $\omega$. Então a imagem de u pelo homomorfismo

$$
H_{2}\left(T_{2}\right)^{\sigma} \longrightarrow \frac{H_{3}\left(G M_{2}(F)\right)}{H_{3}\left(T_{2}\right)}
$$

coincide com a classe do ciclo $b-\varphi_{\omega}^{\prime}(h)$. 
Demonstração. Considere o seguinte diagrama

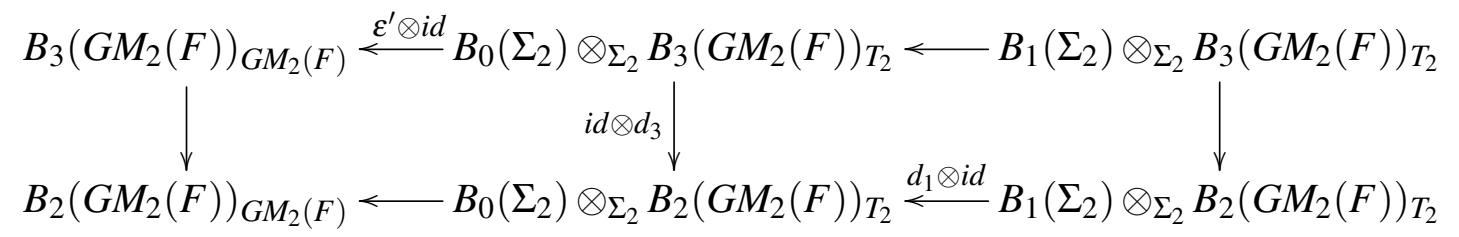

Se $h=\left[g_{1} \mid g_{2}\right]-\left[g_{2} \mid g_{1}\right]$ para algums $g_{1}, g_{2} \in T_{2}$, então $\omega \otimes h$ representa $u$ e temos

$$
\left(i d \otimes d_{3}\right)\left(\omega[] \otimes b-[] \otimes \varphi_{\omega}^{\prime}(h)\right)=\omega[] \otimes h-[] \otimes h=\left(d_{1} \otimes i d\right)([\omega] \otimes h) .
$$

Agora tem-se $\varepsilon^{\prime} \otimes i d\left(\omega[] \otimes b-[] \otimes \varphi_{\omega}^{\prime}(h)\right)$ representa a imagem de $u$ em $\frac{F_{2}^{\prime} H_{3}\left(G M_{2}(F)\right)}{H_{3}\left(T_{2}\right)}$. Mas

$$
\left(\varepsilon^{\prime} \otimes i d\right)\left(\omega[] \otimes b-[] \otimes \varphi_{\omega}^{\prime}(h)\right)=b-\varphi_{\omega}^{\prime}(h) \in B_{3}\left(G M_{2}(F)\right)_{G M_{2}(F)} .
$$

Então $b-\varphi_{\omega}^{\prime}(h)$ representa a imagem de $u$ em $\frac{H_{3}\left(G M_{2}(F)\right)}{H_{3}\left(T_{2}\right)}$.

TEOREMA 4.1. Seja F um corpo infinito. Então temos a sequência exata

$$
H_{3}\left(G M_{2}(F)\right) \rightarrow H_{3}\left(G L_{2}(F)\right) \rightarrow B(F) \rightarrow 0 .
$$

Demonstração. Pelo Lema (4.3) temos que $\frac{F_{2}^{\prime} H_{3}\left(G M_{2}(F)\right)}{H_{3}\left(T_{2}\right)}=\operatorname{Im}\left(H_{2}\left(T_{2}\right)^{\sigma} \rightarrow \frac{H_{3} G M_{2}(F)}{H_{3}\left(T_{2}\right)}\right)$ e pelos Lemas (4.1), (4.3) e considerando o homomorfismo induzido pela inclusão $G M_{2}(F) \rightarrow$ $G L_{2}(F)$, obtemos um homomorfismo sobrejetor

$$
F_{2}^{\prime} H_{3}\left(G M_{2}(F)\right) \longrightarrow F_{2} H_{3}\left(G L_{2}(F)\right)
$$

Com isto, usando a sequência exata (4.3), obtemos a sequência exata

$$
F_{2}^{\prime} H_{3}\left(G M_{2}(F)\right) \longrightarrow H_{3}\left(G L_{2}(F)\right) \longrightarrow B(F) \longrightarrow 0 \text {. }
$$

Por outro lado, considerando o homomorfismo

$$
\begin{aligned}
i_{*}: H_{3}\left(\Sigma_{2}\right)=H_{3}\left(B \bullet\left(\Sigma_{2}\right)_{\Sigma_{2}}\right) & \longrightarrow H_{3}\left(G L_{2}(F)\right)=H_{3}\left(B \bullet\left(G L_{2}(F)\right)_{G L_{2}(F)}\right), \\
{[\omega|\omega| \omega] } & \longmapsto[\omega|\omega| \omega]
\end{aligned}
$$

induzido pela inclusão $\Sigma_{2} \stackrel{i}{\rightarrow} G L_{2}(F)$, onde $\omega=\left(\begin{array}{ll}0 & 1 \\ 1 & 0\end{array}\right)$ e o automorfismo (4.5)

$$
\begin{aligned}
\left(\tau_{A}\right) \bullet: B \bullet\left(G L_{2}(F)\right)_{G L_{2}(F)} & \longrightarrow B \bullet\left(G L_{2}(F)\right)_{G L_{2}(F)}, \\
{\left[g_{1}|\cdots| g_{n}\right] } & \longmapsto\left[A g_{1} A^{-1}|\cdots| A g_{n} A^{-1}\right]
\end{aligned}
$$

onde $A=\left(\begin{array}{cc}0 & 1 \\ 1 & -1\end{array}\right) \in G L_{2}(F)$, temos em particular

$$
\tau_{A}([\omega|\omega| \omega])=\left[A \omega A^{-1}\left|A \omega A^{-1}\right| A \omega A^{-1}\right]=\left[A^{\prime}\left|A^{\prime}\right| A^{\prime}\right],
$$


onde $A^{\prime}=\left(\begin{array}{cc}1 & 1 \\ 0 & -1\end{array}\right) \in B_{2}$. Usando o isomorfismo do Exemplo (1.30) tem-se que $H_{3}\left(B_{2}\right) \cong$ $H_{3}\left(T_{2}\right)$. Isto implica que $\operatorname{Im}\left(H_{3}\left(B_{2}\right) \rightarrow H_{3}\left(G L_{2}(F)\right)\right)=\operatorname{Im}\left(H_{3}\left(T_{2}\right) \rightarrow H_{3}\left(G L_{2}(F)\right)\right)$. Então $\operatorname{Im}\left(H_{3}\left(\Sigma_{2}\right) \stackrel{i_{*}}{\rightarrow} H_{3}\left(G L_{2}(F)\right)\right) \subseteq \operatorname{Im}\left(H_{3}\left(T_{2}\right) \rightarrow H_{3}\left(G L_{2}(F)\right)\right) \subseteq \operatorname{Im}\left(F_{2}^{\prime} H_{3}\left(G M_{2}(F)\right) \longrightarrow H_{3}\left(G L_{2}(F)\right)\right)$.

Logo, pela decomposição (4.10) obtemos a sequência exata

$$
H_{3}\left(G M_{2}(F)\right) \longrightarrow H_{3}\left(G L_{2}(F)\right) \longrightarrow B(F) \longrightarrow 0 .
$$

Pelo Lema (1.5), temos a seguinte sequência exata

$$
0 \longrightarrow M \stackrel{\cup}{\longrightarrow} H_{3}\left(T_{2}\right) \longrightarrow \operatorname{Tor}_{1}^{\mathbb{Z}}\left(F^{\times}, F^{\times}\right) \longrightarrow 0,
$$

que cinde canonicamente, onde

$$
M:=H_{3}\left(F^{\times}\right) \oplus H_{3}\left(F^{\times}\right) \oplus\left(F^{\times} \otimes_{\mathbb{Z}} H_{2}\left(F^{\times}\right)\right) \oplus\left(H_{2}\left(F^{\times}\right) \otimes_{\mathbb{Z}} F^{\times}\right) \subseteq H_{3}\left(T_{2}\right) .
$$

Agora pelo Lema (1.3), $\operatorname{Tor}_{1}^{\mathbb{Z}}\left(F^{\times}, F^{\times}\right) \cong \operatorname{Tor}_{1}^{\mathbb{Z}}(\mu(F), \mu(F))$, lembrando que

$$
\mu(F)=\left\{a \in F: \text { existe } n \text { tal que } a^{n}=1\right\}
$$

Aplicando o funtor $-\Sigma_{2}$ sobre a sequência (4.13) obtemos a sequência exata

$$
0 \longrightarrow M_{\Sigma_{2}} \longrightarrow H_{3}\left(T_{2}\right)_{\sigma} \longrightarrow \operatorname{Tor}_{1}^{\mathbb{Z}}(\mu(F), \mu(F))_{\Sigma_{2}} \longrightarrow 0
$$

e usando o Lema da serpente sobre o diagrama comutativo

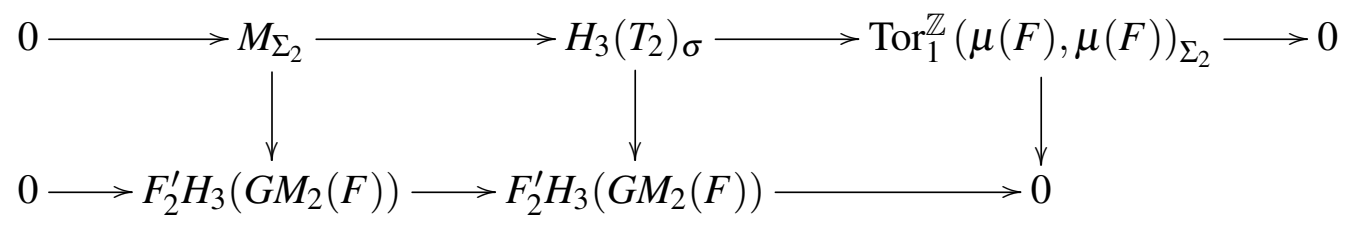

obtemos a seguinte sequência exata

$$
\operatorname{Tor}_{1}^{\mathbb{Z}}(\mu(F), \mu(F))_{\Sigma_{2}} \longrightarrow \frac{F_{2}^{\prime} H_{3}\left(G M_{2}(F)\right)}{M} \longrightarrow \frac{F_{2}^{\prime} H_{3}\left(G M_{2}(F)\right)}{H_{3}\left(T_{2}\right)} \longrightarrow 0 .
$$

Definimos o quociente

$$
T_{F}:=\frac{F_{2}^{\prime} H_{3}\left(G M_{2}(F)\right)}{M} .
$$

Dado que $H_{1}\left(\Sigma_{2}, F^{\times} \otimes_{\mathbb{Z}} F^{\times}\right)=\frac{F_{2}^{\prime} H_{3}\left(G M_{2}(F)\right)}{H_{3}\left(T_{2}\right)}$, então pelo Exemplo (1.15) temos a sequência exata

$$
\operatorname{Tor}_{1}^{\mathbb{Z}}(\mu(F), \mu(F))_{\Sigma_{2}} \longrightarrow T_{F} \longrightarrow H_{1}\left(\Sigma_{2}, \mu_{2^{\infty}}(F) \otimes_{\mathbb{Z}} \mu_{2^{\infty}}(F)\right) \longrightarrow 0 .
$$


Agora pela sequência (4.8) e usando a sequência (4.14) temos a injetividade no lado esquerdo

$$
0 \longrightarrow \operatorname{Tor}_{1}^{\mathbb{Z}}(\mu(F), \mu(F))_{\Sigma_{2}} \longrightarrow T_{F} \longrightarrow H_{1}\left(\Sigma_{2}, \mu_{2^{\infty}}(F) \otimes_{\mathbb{Z}} \mu_{2^{\infty}}(F)\right) \longrightarrow 0 .
$$

Note também que pelo Exemplo (1.5)

$$
\operatorname{Tor}_{1}^{\mathbb{Z}}(\mu(F), \mu(F))_{\Sigma_{2}}=\lim _{\rightarrow} \operatorname{Tor}_{1}^{\mathbb{Z}}\left(\mu_{n}(F), \mu_{n}(F)\right)_{\Sigma_{2}} \cong \lim _{\rightarrow} \operatorname{Tor}_{1}^{\mathbb{Z}}\left(\mu_{n}(F), \mu_{n}(F)\right) \cong \operatorname{Tor}_{1}^{\mathbb{Z}}(\mu(F), \mu(F)),
$$

pois $\quad \Sigma_{2}$ age trivialmente sobre $\operatorname{Tor}_{1}^{\mathbb{Z}}\left(\mu_{n}(F), \mu_{n}(F)\right) \cong \mu_{n}(F)$. Por outro lado, se $a \otimes b \in \mu_{2^{\infty}}(F) \otimes_{\mathbb{Z}} \mu_{2^{\infty}}(F)$, então existe $n$ inteiro tal que $a^{2^{n}}=b^{2^{n}}=1$, isto é, $a, b \in\langle\xi\rangle$, onde $\xi \in F^{\times}$é uma $2^{n}$-raiz primitiva da unidade. Como

$$
a \otimes b=\xi^{k} \otimes \xi^{l}=k l(\xi \otimes \xi),
$$

para alguns inteiros $k$ e $l$, então $\sigma(a \otimes b)=-k l(\xi \otimes \xi)=-a \otimes b$. Assim pelo Exemplo (1.15)

$$
H_{1}\left(\Sigma_{2}, \mu_{2^{\infty}}(F) \otimes_{\mathbb{Z}} \mu_{2^{\infty}}(F)\right)={ }_{2}\left(\mu_{2^{\infty}}(F) \otimes_{\mathbb{Z}} \mu_{2^{\infty}}(F)\right) .
$$

Portanto obtemos a sequência exata

$$
0 \longrightarrow \operatorname{Tor}_{1}^{\mathbb{Z}}(\mu(F), \mu(F)) \longrightarrow T_{F} \longrightarrow 2\left(\mu_{2^{\infty}}(F) \otimes_{\mathbb{Z}} \mu_{2^{\infty}}(F)\right) \longrightarrow 0 .
$$

Pelo Exemplo (1.22) se char $(F)=2$, então ${ }_{2}\left(\mu_{2^{\infty}}(F) \otimes_{\mathbb{Z}} \mu_{2^{\infty}}(F)\right)=0$. Seja $\mu_{2^{\infty}}(F)$ infinito. Então $\mu_{2^{n}}(F) \subseteq \mu_{2^{n+1}}(F)=\langle\eta\rangle \operatorname{com} \eta \in F^{\times}$uma $2^{n+1}$-ésima raiz primitiva da unidade e $\xi=\eta^{2}$ e $\log 0$

$$
a \otimes b=k l(\xi \otimes \xi)=k l\left(\eta^{2} \otimes \eta^{2}\right)=2 k l(2 \eta \otimes \eta)=0
$$

obtendo assim

$$
{ }_{2}\left(\mu_{2^{\infty}}(F) \otimes_{\mathbb{Z}} \mu_{2^{\infty}}(F)\right)=0 .
$$

Agora se $\operatorname{char}(F) \neq 2$ e se $\mu_{2^{\infty}}(F)$ é finito, então $\mu_{2^{\infty}}(F)=\mu_{2^{n}}(F)$ para algum inteiro $n$. Considere $m$ um inteiro tal que $2^{n}=2 m$, então

$$
2\left(\mu_{2^{\infty}}(F) \otimes_{\mathbb{Z}} \mu_{2^{\infty}}(F)\right)=\{0,(-1) \otimes \xi\} \cong \mathbb{Z} / 2 \mathbb{Z},
$$

onde $-1=\xi^{m}$. Portanto

$$
{ }_{2}\left(\mu_{2^{\infty}}(F) \otimes_{\mathbb{Z}} \mu_{2^{\infty}}(F)\right) \cong \begin{cases}0 & \text { se } \operatorname{char}(F)=2 \text { ou se } \mu_{2^{\infty}}(F) \text { for infinito, } \\ \mathbb{Z} / 2 \mathbb{Z} & \text { se } \operatorname{char}(F) \neq 2 \text { e } \mu_{2^{\infty}}(F) \text { for finito. }\end{cases}
$$

LEMA 4.4. Seja $F$ um corpo infinito tal que char $(F) \neq 2$ e $\mu_{2^{\infty}}(F)$ é finito. Então a sequência exata

$$
0 \longrightarrow \operatorname{Tor}_{1}^{\mathbb{Z}}(\mu(F), \mu(F)) \longrightarrow T_{F} \longrightarrow \mathbb{Z} / 2 \mathbb{Z} \longrightarrow 0
$$

não cinde e $T_{F}$ é a única extensão não trivial de $\mathbb{Z} / 2 \mathbb{Z}$ por $\operatorname{Tor}_{1}^{\mathbb{Z}}(\mu(F), \mu(F))$. 
Demonstração. A sequência (4.17) é dada por

$$
0 \longrightarrow \frac{H_{3}\left(T_{2}\right)}{M} \longrightarrow \frac{F_{2}^{\prime} H_{3}\left(G M_{2}(F)\right)}{M} \longrightarrow \frac{F_{2}^{\prime} H_{3}\left(G M_{2}(F)\right)}{H_{3}\left(T_{2}\right)} \longrightarrow 0
$$

Seja $\mu_{2^{\infty}}(F)=\mu_{n}(F)=\langle\xi\rangle$ e seja $\xi^{m}=-1$, onde $n=2 m=2^{r}$. Se $B \bullet\left(G M_{2}(F)\right)$ é a resolução barra de $\mathbb{Z}$ sobre $G M_{2}(F)$, então a imagem de $(-1) \otimes \xi$ em $H_{2}\left(T_{2}\right)^{\sigma}$ será representada pelo ciclo $h:=[(-1,1) \mid(1, \xi)]-[(1, \xi) \mid(-1,1)] \in B_{2}\left(T_{2}\right)_{T_{2}}$. Considere o automorfismo (4.5), $\tau_{\omega}^{\prime}$ : $B_{2}\left(G M_{2}(F)\right)_{T_{2}} \rightarrow B_{3}\left(G M_{2}(F)\right)_{T_{2}}$ onde $\omega=\left(\begin{array}{ll}0 & 1 \\ 1 & 0\end{array}\right)$. É claro que

$$
\tau_{\omega}^{\prime}(h)=[(1,-1) \mid(\xi, 1)]-[(\xi, 1) \mid(1,-1)] .
$$

Por outro lado, se $b \in B_{3}\left(T_{2}\right)_{2}$,

$$
\begin{aligned}
b:= & {[(1,-1)|(1,-1)|(\xi, 1)]-[(1,-1)|(\xi, 1)|(1,-1)]+[(\xi, 1)|(1,-1)|(1,-1)] } \\
& +\sum_{i=1}^{m-1}\left(\left[(\xi, 1)|(1, \xi)|\left(1, \xi^{i}\right)\right]-\left[(1, \xi)|(\xi, 1)|\left(1, \xi^{i}\right)\right]+\left[(1, \xi)\left|\left(1, \xi^{i}\right)\right|(\xi, 1)\right]\right. \\
& \left.+\left[(\xi, 1)\left|\left(\xi^{i}, 1\right)\right|(1, \xi)\right]-\left[(1, \xi)|(1, \xi)|\left(\xi^{i}, 1\right)\right]+\left[(1, \xi)|(\xi, 1)|\left(\xi^{i}, 1\right)\right]\right) .
\end{aligned}
$$

então

$$
\begin{aligned}
d_{3}(b)= & \tau_{\omega}^{\prime}(h)-h+[(-1,1) \mid(1, \xi)]-[(1, \xi) \mid(-1,1)]+[(1,-1) \mid(\xi, 1)]-[(\xi, 1) \mid(1,-1)] \\
& +\sum_{i=1}^{m-1} d_{3}\left(\left[(\xi, 1)|(1, \xi)|\left(1, \xi^{i}\right)\right]-\left[(1, \xi)|(\xi, 1)|\left(1, \xi^{i}\right)\right]+\left[(1, \xi)\left|\left(1, \xi^{i}\right)\right|(\xi, 1)\right]\right. \\
& \left.+\left[(\xi, 1)\left|\left(\xi^{i}, 1\right)\right|(1, \xi)\right]-\left[(1, \xi)|(1, \xi)|\left(\xi^{i}, 1\right)\right]+\left[(1, \xi)|(\xi, 1)|\left(\xi^{i}, 1\right)\right]\right) \\
= & \tau_{\omega}^{\prime}(h)-h .
\end{aligned}
$$

Agora pelo Lema (4.3), o ciclo $b-\varphi_{\omega}^{\prime}(h)$ é a imagem de $(-1) \otimes \xi$ em $\frac{F_{2}^{\prime} H_{3}\left(G M_{2}(F)\right)}{H_{3}\left(T_{2}\right)}$. Note que

$$
\begin{aligned}
\varphi_{\omega}^{\prime}(h)= & {[\omega|(1,-1)|(\xi, 1)]-[(-1,1)|\omega|(\xi, 1)]+[(-1,1)|(1, \xi)| \omega] } \\
& -[\omega|(\xi, 1)|(1,-1)]+[(1, \xi)|\omega|(1,-1)]-[(1, \xi)|(-1,1)| \omega] .
\end{aligned}
$$

Para provar que a sequência exata não cinde é suficiente provar que

$$
2 \overline{\left(b-\varphi_{\omega}^{\prime}(h)\right)}=\overline{\chi(\xi)} \in F_{2}^{\prime} H_{3}\left(G M_{2}(F)\right) \subseteq H_{3}\left(G M_{2}(F)\right)
$$

onde pelo Lema (1.5), $\chi(\xi)$ é a imagem de $\langle\xi, n, \xi\rangle \in \operatorname{Tor}_{1}^{\mathbb{Z}}(\mu(F), \mu(F))$ via a inclusão $\operatorname{Tor}_{1}^{\mathbb{Z}}(\mu(F), \mu(F)) \cong H_{3}\left(T_{2}\right) / M \hookrightarrow F_{2}^{\prime} H_{3}\left(G M_{2}(F)\right) / M$. Se consideramos os seguintes elementos em $B_{4}\left(G M_{2}(F)\right)_{G M_{2}(F)}$

$$
\begin{aligned}
a_{\xi}:= & {[\omega|(1,-1)|(1,-1) \mid(\xi, 1)]-[\omega|(1,-1)|(\xi, 1) \mid(1,-1)]+[(-1,1)|\omega|(\xi, 1) \mid(1,-1)] } \\
& -[(-1,1)|(1, \xi)| \omega \mid(1,-1)]+[(-1,1)|(1, \xi)|(-1,1) \mid \omega]-[(1, \xi)|\omega|(1,-1) \mid(1,-1)] \\
& +[\omega|(\xi, 1)|(1,-1) \mid(1,-1)]-[(-1,1)|\omega|(1,-1) \mid(\xi, 1)]+[(1, \xi)|(-1,1)| \omega \mid(1,-1)] \\
& -[(1, \xi)|(-1,1)|(-1,1) \mid \omega]+[(-1,1)|(-1,1)| \omega \mid(\xi, 1)]-[(-1,1)|(-1,1)|(1, \xi) \mid \omega],
\end{aligned}
$$




$$
\begin{aligned}
b_{\xi}:= & \sum_{i=1}^{m-1}\left(\left[(\xi, 1)|(1, \xi)|\left(1, \xi^{i}\right) \mid(1,-1)\right]-\left[(1, \xi)|(\xi, 1)|\left(1, \xi^{i}\right) \mid(1,-1)\right]\right. \\
& -\left[(1, \xi)\left|\left(1, \xi^{i}\right)\right|(1,-1) \mid(\xi, 1)\right]+\left[(1, \xi)\left|\left(1, \xi^{i}\right)\right|(\xi, 1) \mid(1,-1)\right] \\
& +\left[(1, \xi)|(\xi, 1)|\left(\xi^{i}, 1\right) \mid(-1,1)\right]-\left[(\xi, 1)|(1, \xi)|\left(\xi^{i}, 1\right) \mid(-1,1)\right] \\
& \left.-\left[(\xi, 1)\left|\left(\xi^{i}, 1\right)\right|(-1,1) \mid(1, \xi)\right]+\left[(\xi, 1)\left|\left(\xi^{i}, 1\right)\right|(1, \xi) \mid(-1,1)\right]\right),
\end{aligned}
$$

então temos

$$
\begin{aligned}
d_{4}\left(a_{\xi}\right)= & \left.b-2 \varphi_{\omega}^{\prime}(h)\right)+[(-1,1)|(1, \xi)|(-1,1)]-[(-1,1)|(-1,1)|(1, \xi)]-[(1, \xi)|(-1,1)|(-1,1)] \\
& -\sum_{i=1}^{m-1}\left(\left[(\xi, 1)|(1, \xi)|\left(1, \xi^{i}\right)\right]-\left[(1, \xi)|(\xi, 1)|\left(1, \xi^{i}\right)\right]+\left[(1, \xi)\left|\left(1, \xi^{i}\right)\right|(\xi, 1)\right]\right. \\
& {\left.\left[(\xi, 1)\left|\left(\xi^{i}, 1\right)\right|(1, \xi)\right]-\left[(\xi, 1)|(1, \xi)|\left(\xi^{i}, 1\right)\right]+\left[(1, \xi)|(\xi, 1)|\left(\xi^{i}, 1\right)\right]\right), } \\
d_{4}\left(b_{\xi}\right)= & b-\chi(\xi)-[(-1,1)|(1, \xi)|(-1,1)]+[(-1,1)|(-1,1)|(1, \xi)]+[(1, \xi)|(-1,1)|(-1,1)] \\
& +\sum_{i=1}^{m-1}\left(\left[(\xi, 1)|(1, \xi)|\left(1, \xi^{i}\right)\right]-\left[(1, \xi)|(\xi, 1)|\left(1, \xi^{i}\right)\right]+\left[(1, \xi)\left|\left(1, \xi^{i}\right)\right|(\xi, 1)\right]\right. \\
& {\left.\left[(\xi, 1)\left|\left(\xi^{i}, 1\right)\right|(1, \xi)\right]-\left[(\xi, 1)|(1, \xi)|\left(\xi^{i}, 1\right)\right]+\left[(1, \xi)|(\xi, 1)|\left(\xi^{i}, 1\right)\right]\right), }
\end{aligned}
$$

Logo $\left.d_{4}\left(a_{\xi}+b_{\xi}\right)+\chi(\xi)=2\left(b-\varphi_{\omega}^{\prime}(h)\right)\right)$, isto é, $2 \overline{\left.\left(b-\varphi_{\omega}^{\prime}(h)\right)\right)}=\overline{\chi(\xi)} \in F_{2}^{\prime} H_{3}\left(G M_{2}(F)\right)$. Portanto a sequência (4.17) não cinde e pelo Exemplo (1.22), é a única a menos de isomorfismo.

\subsection{Terceira homologia de $G L_{3}$ de um corpo}

Seja $F$ um corpo. Para $n \geq 0$ seja $X_{n}$ o conjunto de todas as $(n+1)-$ uplas $\left(\left\langle v_{0}\right\rangle, \ldots,\left\langle v_{n}\right\rangle\right)$ tais que cada $\min \{n, 3\}$ vetores de $v_{0}, \ldots, v_{n} \in F^{3}$ são linearmente independentes. Seja $C_{n}\left(F^{3}\right):=\mathbb{Z} X_{n} \mathrm{o}$ $\mathbb{Z}$-módulo livre gerado por $X_{n}$. Considere o homomorfismo

$$
\begin{aligned}
\varepsilon: C_{0}\left(F^{3}\right) & \longrightarrow \mathbb{Z}, \\
\sum_{j} m_{j}\left(\left\langle v_{0}^{j}\right\rangle\right) & \longmapsto \sum_{j} m_{j}
\end{aligned}
$$

e para $n \geq 1$, os homomorfismos

$$
\begin{aligned}
\partial_{n}: C_{n}\left(F^{3}\right) & \longrightarrow C_{n-1}\left(F^{3}\right), \\
\sum_{j} m_{j}\left(\left\langle v_{0}^{j}\right\rangle, \ldots,\left\langle v_{n}^{j}\right\rangle\right) & \longmapsto \sum_{j} m_{j}\left(\sum_{k=0}^{n}(-1)^{k}\left(\left\langle v_{0}^{j}\right\rangle, \ldots, \widehat{\left\langle v_{k}^{j}\right\rangle}, \ldots,\left\langle v_{n}^{j}\right\rangle\right)\right),
\end{aligned}
$$

onde

$$
\left(\left\langle v_{0}^{j}\right\rangle, \ldots, \widehat{\left\langle v_{k}^{j}\right\rangle}, \ldots,\left\langle v_{n}^{j}\right\rangle\right)=\left(\left\langle v_{0}^{j}\right\rangle, \ldots,\left\langle v_{k-1}^{j}\right\rangle,\left\langle v_{k+1}^{j}\right\rangle, \ldots,\left\langle v_{n}^{j}\right\rangle\right)
$$

LEMA 4.5. A sequência

$$
C_{\bullet}\left(F^{3}\right) \stackrel{\varepsilon}{\longrightarrow} \mathbb{Z}: \quad \cdots \rightarrow C_{n}\left(F^{3}\right) \stackrel{\partial_{n}}{\longrightarrow} C_{n-1}\left(F^{3}\right) \rightarrow \cdots \rightarrow C_{0}\left(F^{3}\right) \stackrel{\varepsilon}{\longrightarrow} \mathbb{Z} \rightarrow 0,
$$

é um complexo de cadeias. Além disso, se $F$ for infinito, então o complexo $C_{\bullet}\left(F^{3}\right) \stackrel{\varepsilon}{\longrightarrow} \mathbb{Z}$ é exato. 
Demonstração. De forma análoga ao Lema (2.6).

Definimos para todo $n \geq 0$, uma ação do grupo $G L_{3}(F)$ sobre $X_{n}$ como segue,

$$
A \cdot\left(\left\langle v_{0}\right\rangle, \ldots,\left\langle v_{n}\right\rangle\right):=\left(\left\langle A v_{0}\right\rangle, \ldots,\left\langle A v_{n}\right\rangle\right),
$$

onde $A \in G L_{3}(F),\left(\left\langle v_{0}\right\rangle, \ldots,\left\langle v_{n}\right\rangle\right) \in X_{n}$. Logo

$$
C_{n}\left(F^{3}\right)=\mathbb{Z} X_{n} \cong \bigoplus_{x \in T_{n}}\left(\mathbb{Z} G L_{3}(F) \otimes_{\left(G L_{3}(F)\right)_{x}} \mathbb{Z}\right)
$$

onde $T_{n}$ é um conjunto de representantes das órbitas da ação de $G L_{3}(F)$ sobre $X_{n}$ e $\left(G L_{3}(F)\right)_{x}$ é o subgrupo estabilizador de $x \in T_{n}$. É fácil verificar que o grupo $G L_{3}(F)$ age transitivamente sobre $X_{n}$ para $n=0,1,2,3$. Sejam

$$
\begin{aligned}
& x_{0}:=\left(\left\langle e_{1}\right\rangle\right) \in X_{0} \operatorname{com} e_{1}=\left(\begin{array}{l}
1 \\
0 \\
0
\end{array}\right), \\
& x_{1}:=\left(\left\langle e_{1}\right\rangle,\left\langle e_{2}\right\rangle\right) \in X_{1} \operatorname{com} e_{2}=\left(\begin{array}{l}
0 \\
1 \\
0
\end{array}\right), \\
& x_{2}:=\left(\left\langle e_{1}\right\rangle,\left\langle e_{2}\right\rangle,\left\langle e_{3}\right\rangle\right) \in X_{2} \operatorname{com} e_{3}=\left(\begin{array}{l}
0 \\
0 \\
1
\end{array}\right), \\
& x_{3}:=\left(\left\langle e_{1}\right\rangle,\left\langle e_{2}\right\rangle,\left\langle e_{3}\right\rangle,\left\langle e_{1}+e_{2}+e_{3}\right\rangle\right) \in X_{3} .
\end{aligned}
$$

Então para $0 \leq i \leq 3, X_{i}=o\left(x_{i}\right)$. Note que para o caso $n=4$ e $n=5$ facilmente podemos mostrar que

$$
X_{4}=\bigsqcup_{\substack{a, x \in F^{\times}-\{1\} \\ a \neq x}} o\left(x_{a, x}\right), \quad X_{5}=\bigsqcup_{\substack{a, b, x, y \in F^{\times}-\{1\} \\ a \neq x, b \neq y, a \neq b, x \neq y, a y \neq b x}} o\left(x_{a, b, x, y}\right),
$$

onde

$$
\begin{gathered}
x_{a, x}=\left(\left\langle e_{1}\right\rangle,\left\langle e_{2}\right\rangle,\left\langle e_{1}+e_{2}+e_{3}\right\rangle,\left\langle e_{1}+a e_{2}+x e_{3}\right\rangle,\left\langle e_{3}\right\rangle\right) \in X_{4}, \\
x_{a, b, x, y}=\left(\left\langle e_{1}\right\rangle,\left\langle e_{2}\right\rangle,\left\langle e_{1}+e_{2}+e_{3}\right\rangle,\left\langle e_{1}+a e_{2}+x e_{3}\right\rangle,\left\langle e_{1}+b e_{2}+y e_{3}\right\rangle,\left\langle e_{3}\right\rangle\right) \in X_{5} .
\end{gathered}
$$

Portanto para $0 \leq n \leq 3, C_{n}\left(F^{3}\right)_{G L_{3}(F)} \cong \mathbb{Z}$. Para $n=4$, temos

$$
C_{4}\left(F^{3}\right)_{G L_{3}(F)} \cong \bigoplus_{\substack{a, x \in F^{\times}-\{1\} \\
a \neq x}} \mathbb{Z}\left[\begin{array}{l}
a \\
x
\end{array}\right]
$$

onde $\left[\begin{array}{l}a \\ x\end{array}\right]$ representa a órbita de $x_{a, x}$ e para $n=5$, temos

$$
C_{5}\left(F^{3}\right)_{G L_{3}(F)} \cong \bigoplus_{\substack{a, b, x, y \in F^{\times}-\{1\} \\
a \neq x, b \neq y, a \neq b, x \neq y, a y \neq b x}} \mathbb{Z}\left[\begin{array}{ll}
a & b \\
x & y
\end{array}\right]
$$


onde $\left[\begin{array}{ll}a & b \\ x & y\end{array}\right]$ representa a órbita de $x_{a, b, x, y}$.

Por outro lado, defina o complexo $C_{\bullet}^{a}\left(F^{3}\right)$ de $G L_{3}(F)$ - módulos como sendo, para cada $n \geq 0$, $C_{n}^{a}\left(F^{3}\right):=C_{n}\left(F^{3}\right)$ e defina para $n \geq 1$ os $G L_{3}(F)$-homomorfismos $\partial_{n}^{a}: C_{n}^{a}\left(F^{3}\right) \rightarrow C_{n-1}^{a}\left(F^{3}\right)$ como sendo

$$
\begin{aligned}
\partial_{n}^{a}: C_{n}^{a}\left(F^{3}\right) & \longrightarrow C_{n-1}^{a}\left(F^{3}\right), \\
\left(\left\langle v_{0}\right\rangle, \ldots,\left\langle v_{n}\right\rangle\right) & \longmapsto \sum_{i=0}^{n-1}(-1)^{i}\left(\left\langle v_{0}\right\rangle, \ldots, \widehat{\left\langle v_{i}\right\rangle}, \ldots,\left\langle v_{n}\right\rangle\right) .
\end{aligned}
$$

LEMA 4.6. A sequência:

$$
C_{\bullet}^{a}\left(F^{3}\right): \quad \cdots \rightarrow C_{n}^{a}\left(F^{3}\right) \stackrel{\partial_{n}^{a}}{\longrightarrow} C_{n-1}^{a}\left(F^{3}\right) \rightarrow \cdots \rightarrow C_{1}^{a}\left(F^{3}\right) \stackrel{\partial_{1}^{a}}{\longrightarrow} C_{0}^{a}\left(F^{3}\right) \rightarrow 0,
$$

é um complexo de cadeias. Além disso, se $F$ for infinito, então o complexo $C_{\bullet}^{a}\left(F^{3}\right)$ é exato.

Demonstração. De forma análoga ao Lema (2.6).

Defina para $n \geq 1$ os $G L_{3}(F)$-homomorfismos

$$
\begin{aligned}
\gamma_{n}: C_{n}\left(F^{3}\right) & \longrightarrow C_{n}^{a}\left(F^{3}\right), \\
\left(\left\langle v_{0}\right\rangle, \ldots,\left\langle v_{n}\right\rangle\right) & \longmapsto \sum_{k=0}^{n}(-1)^{k n}\left(\left\langle v_{k}\right\rangle,\left\langle v_{k+1}\right\rangle, \ldots,\left\langle v_{k+n}\right\rangle\right),
\end{aligned}
$$

onde cada $k+i$ é considerado módulo $n+1$. Note que para $n \geq 2$, temos

$$
\begin{aligned}
\left(\partial_{n}^{a} \circ \gamma_{n}\right)\left(\left\langle v_{0}\right\rangle, \ldots,\left\langle v_{n}\right\rangle\right)= & \partial_{n}^{a}\left(\sum_{k=0}^{n}(-1)^{k n}\left(\left\langle v_{k}\right\rangle, \ldots,\left\langle v_{k+n}\right\rangle\right)\right) \\
= & \sum_{k=0}^{n}(-1)^{k n} \partial_{n}^{a}\left(\left\langle v_{k}\right\rangle, \ldots,\left\langle v_{k+n}\right\rangle\right) \\
= & \sum_{k=0}^{n}(-1)^{k n} \sum_{i=0}^{n-1}(-1)^{i}\left(\widehat{\left\langle v_{k+i}\right\rangle}, \ldots,\left\langle v_{k+n}\right\rangle\right) \\
= & \gamma_{n-1}\left(\left\langle v_{1}\right\rangle,\left\langle v_{2}\right\rangle, \ldots,\left\langle v_{n}\right\rangle\right)-\gamma_{n-1}\left(\left\langle v_{0}\right\rangle,\left\langle v_{2}\right\rangle, \ldots,\left\langle v_{n}\right\rangle\right)+\ldots \\
& +(-1)^{n} \gamma_{n-1}\left(\left\langle v_{0}\right\rangle,\left\langle v_{1}\right\rangle, \ldots,\left\langle v_{n-2}\right\rangle,\left\langle v_{n-1}\right\rangle\right) \\
= & \gamma_{n-1}\left(\left(\left\langle v_{1}\right\rangle,\left\langle v_{2}\right\rangle, \ldots,\left\langle v_{n}\right\rangle\right)-\left(\left\langle v_{0}\right\rangle,\left\langle v_{2}\right\rangle, \ldots,\left\langle v_{n}\right\rangle\right)+\ldots\right. \\
& \left.+(-1)^{n}\left(\left\langle v_{0}\right\rangle,\left\langle v_{1}\right\rangle, \ldots,\left\langle v_{n-1}\right\rangle\right)\right) \\
= & \gamma_{n-1}\left(\partial_{n}\left(\left\langle v_{0}\right\rangle,\left\langle v_{1}\right\rangle, \ldots,\left\langle v_{n}\right\rangle\right)\right)=\left(\gamma_{n-1} \circ \partial_{n}\right)\left(\left\langle v_{0}\right\rangle, \ldots,\left\langle v_{n}\right\rangle\right) .
\end{aligned}
$$

Portanto temos o seguinte diagrama comutativo

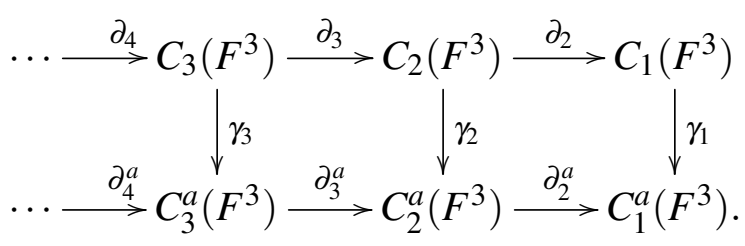


Considere $D_{\bullet}\left(F^{3}\right)$ o mapping cone do complexo $C_{\bullet}^{a}\left(F^{3}\right)$, isto é $D_{\bullet}\left(F^{3}\right)$ é complexo de cadeias feito dos seguintes $G L_{3}(F)$-módulos

$$
D_{n}\left(F^{3}\right):=0, \text { se } n<-1 \quad D_{-1}\left(F^{3}\right):=\mathbb{Z}, \quad D_{0}\left(F^{3}\right):=C_{1}^{a}\left(F^{3}\right),
$$

e para $n \geq 1$,

$$
D_{n}\left(F^{3}\right):=C_{n}\left(F^{3}\right) \oplus C_{n+1}^{a}\left(F^{3}\right) .
$$

junto com os $G L_{3}(F)$-homomorfismos definidos como segue

$$
\begin{aligned}
\varepsilon_{D}: D_{0}\left(F^{3}\right) & \longrightarrow \mathbb{Z}, \\
\sum_{i} n_{i}\left(\left\langle v_{0}^{i}\right\rangle,\left\langle v_{1}^{i}\right\rangle\right) & \longmapsto \sum_{i} n_{i}, \\
\delta_{1}=\gamma_{1}+\partial_{2}^{a}: D_{1}\left(F^{3}\right) & \rightarrow D_{0}\left(F^{3}\right),
\end{aligned}
$$

e para $n \geq 2$,

$$
\delta_{n}:=\left[\begin{array}{cc}
-\partial_{n} & 0 \\
\gamma_{n} & \partial_{n+1}^{a}
\end{array}\right]: D_{n}\left(F^{3}\right) \rightarrow D_{n-1}\left(F^{3}\right),
$$

Obtendo assim a sequência de $G L_{3}(F)$-módulos

$D_{\bullet}\left(F^{3}\right) \stackrel{\varepsilon_{D}}{\longrightarrow} \mathbb{Z}: \quad \cdots \rightarrow D_{n}\left(F^{3}\right) \stackrel{\delta_{n}}{\longrightarrow} D_{n-1}\left(F^{3}\right) \rightarrow \cdots \rightarrow D_{1}\left(F^{3}\right) \stackrel{\delta_{1}}{\longrightarrow} D_{0}\left(F^{3}\right) \stackrel{\varepsilon_{D}}{\longrightarrow} \mathbb{Z} \rightarrow 0$.

LEMA 4.7. A sequência $D_{\bullet}\left(F^{3}\right) \stackrel{\varepsilon_{D}}{\longrightarrow} \mathbb{Z}$ é um complexo de cadeias de $G L_{3}(F)$-módulos. Se $F$ for infinito, então $D_{\bullet}\left(F^{3}\right) \stackrel{\varepsilon_{D}}{\longrightarrow} \mathbb{Z}$ é exato.

Demonstração. É fácil verificar que $D_{\bullet}\left(F^{3}\right) \rightarrow \mathbb{Z}$ é um complexo de cadeias. Suponha que $F$ for infinito. Seja $x=\sum_{i} n_{i}\left(\left\langle v_{0}^{i}\right\rangle,\left\langle v_{1}^{i}\right\rangle\right) \in C_{1}^{a}\left(F^{3}\right)$ tal que $\sum_{i} n_{i}=0$. Como $F$ é infinito, existe $w \in F^{3}-\{0\}$ tal que $\langle w\rangle \neq\left\langle v_{1}^{i}\right\rangle$, para todo $i$. Se $z=\sum_{i} n_{i}\left(\langle w\rangle,\left\langle v_{1}^{i}\right\rangle\right) \in C_{1}\left(F^{3}\right)$, então $\partial_{1}^{a}\left(\gamma_{1}(z)-\right.$ $x)=0$. Assim, existe $z^{\prime} \in C_{2}^{a}\left(F^{3}\right)$ tal que $x=\gamma_{1}(z)+\partial_{2}^{a}\left(z^{\prime}\right)=\delta_{1}\left(z, z^{\prime}\right) \in \operatorname{Im} \delta_{1}$. Seja $n \geq 1$ e seja $\left(x, x^{\prime}\right) \in D_{n}\left(F^{3}\right)$ tal que $\delta_{n}\left(x, x^{\prime}\right)=(0,0)$. Como $C_{\bullet}\left(F^{3}\right) \rightarrow \mathbb{Z}$ é exato (4.5), existe $y \in C_{n+1}\left(F^{3}\right)$ tal que $-\partial_{n+1}(y)=x$. Como $\gamma_{n}(x)+\partial_{n}^{a}\left(x^{\prime}\right)=0$, temos

$$
\begin{aligned}
0 & =\gamma_{n}(x)+\partial_{n+1}^{a}\left(x^{\prime}\right)=-\gamma_{n}\left(\partial_{n+1}(y)\right)+\partial_{n+1}^{a}\left(x^{\prime}\right) \\
& =\partial_{n+1}^{a}\left(x^{\prime}\right)-\partial_{n+1}^{a}\left(\gamma_{n+1}(y)\right) \\
& =\partial_{n+1}^{a}\left(x^{\prime}-\gamma_{n+1}(y)\right) .
\end{aligned}
$$

Portanto existe $y^{\prime} \in C_{n+2}^{a}\left(F^{3}\right)$ tal que $\partial_{n+2}^{a}\left(y^{\prime}\right)=x^{\prime}-\gamma_{n+1}(y)$, pois $C_{\bullet}^{a}\left(F^{3}\right) \rightarrow \mathbb{Z}$ é exato (4.6). Agora temos

$$
\delta_{n+1}\left(y, y^{\prime}\right)=\left(-\partial_{n+1}(y), \gamma_{n+1}(y)+\partial_{n+2}^{a}\left(y^{\prime}\right)\right)=\left(x, x^{\prime}\right) \in \operatorname{Im} \delta_{n+1},
$$

que implica que $D_{\bullet}\left(F^{3}\right) \rightarrow \mathbb{Z}$ é exato. 


\section{Lembremos que}

$$
\begin{aligned}
& D_{0}\left(F^{3}\right)_{G L_{3}(F)}=C_{1}\left(F^{3}\right)_{G L_{3}(F)} \cong \mathbb{Z} \text {, } \\
& D_{1}\left(F^{3}\right)_{G L_{3}(F)}=C_{1}\left(F^{3}\right)_{G L_{3}(F)} \oplus C_{2}^{a}\left(F^{3}\right)_{G L_{3}(F)} \cong \mathbb{Z} \oplus \mathbb{Z} \text {, } \\
& D_{2}\left(F^{3}\right)_{G L_{3}(F)}=C_{2}\left(F^{3}\right)_{G L_{3}(F)} \oplus C_{3}^{a}\left(F^{3}\right)_{G L_{3}(F)} \cong \mathbb{Z} \oplus \mathbb{Z} \text {, } \\
& D_{3}\left(F^{3}\right)_{G L_{3}(F)}=C_{3}\left(F^{3}\right)_{G L_{3}(F)} \oplus C_{4}^{a}\left(F^{3}\right)_{G L_{3}(F)} \cong \mathbb{Z} \oplus\left(\bigoplus_{\substack{a, x \in F^{\times}-\{1\} \\
a \neq x}} \mathbb{Z}\left[\begin{array}{l}
a \\
x
\end{array}\right]\right) \text {, }
\end{aligned}
$$

$\mathrm{e}$

$$
D_{4}\left(F^{3}\right)_{G L_{3}(F)}=C_{4}\left(F^{3}\right)_{G L_{3}(F)} \oplus C_{5}^{a}\left(F^{3}\right)_{G L_{3}(F)} \cong\left(\bigoplus_{\substack{a, x \in F^{\times}-\{1\} \\
a \neq x}} \mathbb{Z}\left[\begin{array}{l}
a \\
x
\end{array}\right]\right) \bigoplus\left(\bigoplus_{\substack{a, b, x, y \in F^{\times}-\{1\} \\
a \neq x, b \neq y \\
a \neq b, x \neq y \\
a y \neq b x}} \mathbb{Z}\left[\begin{array}{ll}
a & b \\
x & y
\end{array}\right]\right) .
$$

Fazendo os cálculos, o complexo $D \bullet\left(F^{3}\right)_{G L_{3}(F)}$ é dado por

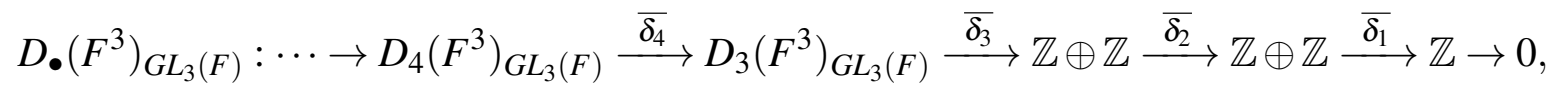

com os diferenciais

$$
\overline{\delta_{1}}=0, \quad \overline{\delta_{2}}=\left[\begin{array}{cc}
-1 & 0 \\
3 & 1
\end{array}\right], \quad \overline{\delta_{3}}=0, \quad \overline{\delta_{4}}=\left[\begin{array}{cc}
-\overline{\partial_{4}} & 0 \\
\overline{\gamma_{4}} & \overline{\partial_{5}^{a}}
\end{array}\right],
$$

onde

$$
\overline{\partial_{4}}: \bigoplus_{\substack{a, x \in F^{\times}-\{1\} \\
a \neq x}} \mathbb{Z}\left[\begin{array}{l}
a \\
x
\end{array}\right] \rightarrow \mathbb{Z}
$$

é dado por $\overline{\partial_{4}}\left(\left[\begin{array}{l}a \\ x\end{array}\right]\right)=1$

$$
\overline{\gamma_{4}}: \bigoplus_{\substack{a, x \in F^{\times}-\{1\} \\
a \neq x}} \mathbb{Z}\left[\begin{array}{l}
a \\
x
\end{array}\right] \rightarrow \bigoplus_{\substack{a, x \in F^{\times}-\{1\} \\
a \neq x}} \mathbb{Z}\left[\begin{array}{l}
a \\
x
\end{array}\right]
$$

é dado por

$$
\overline{\gamma_{4}}\left(\left[\begin{array}{l}
a \\
x
\end{array}\right]\right)=\left[\begin{array}{c}
\frac{x-a}{x} \\
\frac{x-a}{x-1}
\end{array}\right]+\left[\begin{array}{c}
x^{-1} \\
x-a \\
x(1-a)
\end{array}\right]+\left[\begin{array}{c}
\frac{x-1}{x-a} \\
a^{-1}
\end{array}\right]+\left[\begin{array}{c}
\frac{x(1-a)}{x-a} \\
\frac{x}{x-a}
\end{array}\right]+\left[\begin{array}{l}
a \\
x
\end{array}\right]
$$

e

$$
\overline{\partial_{5}^{a}}: \bigoplus_{\substack{a, b, x, y \in F^{\times}-\{1\} \\
a \neq x, b \neq y \\
a \neq b, x \neq y \\
a y \neq b x}} \mathbb{Z}\left[\begin{array}{ll}
a & b \\
x & y
\end{array}\right] \longrightarrow \bigoplus_{\begin{array}{c}
a, x \in F^{\times}-\{1\} \\
a \neq x
\end{array}} \mathbb{Z}\left[\begin{array}{l}
a \\
x
\end{array}\right]
$$


é dado por

$$
\overline{\partial_{5}^{a}}\left(\left[\begin{array}{ll}
a & b \\
x & y
\end{array}\right]\right)=\left[\begin{array}{c}
\frac{1-a}{1-b} \\
\frac{(1-y)(1-a)}{(1-x)(1-b)}
\end{array}\right]-\left[\begin{array}{c}
\frac{1-a^{-1}}{1-b^{-1}} \\
\frac{(1-a)(y-b)}{(1-b)(x-a)}
\end{array}\right]+\left[\begin{array}{l}
\frac{b}{a} \\
\frac{y}{x}
\end{array}\right]-\left[\begin{array}{l}
b \\
y
\end{array}\right]+\left[\begin{array}{l}
a \\
x
\end{array}\right] .
$$

Seja $B_{\bullet}\left(G L_{3}(F)\right) \stackrel{\mathcal{\varepsilon}}{\rightarrow} \mathbb{Z}$ a resolução barra de $\mathbb{Z}$ sobre $G L_{3}(F)$. Pelo Teorema (1.2), existe um morfismo de complexos $\left(B_{\bullet}\left(G L_{3}(F)\right), d_{\bullet}\right) \stackrel{\theta_{\bullet}}{\rightarrow}\left(D_{\bullet}\left(F^{3}\right), \delta_{\bullet}\right)$, único a menos de homotopia, que induz o homomorfismo

$$
\theta: H_{3}\left(G L_{3}(F)\right)=H_{3}\left(B \bullet\left(G L_{3}(F)\right)_{G L_{3}(F)}\right) \longrightarrow H_{3}\left(D_{\bullet}\left(F^{3}\right)_{G L_{3}(F)}\right) .
$$

Com uso do seguinte lema vamos a descrever o homomorfismo

$$
\theta: H_{3}\left(G L_{3}(F)\right) \longrightarrow H_{3}\left(D_{\bullet}\left(F^{3}\right)_{G L_{3}(F)}\right)=\operatorname{coker}\left(D_{4}\left(F^{3}\right)_{G L_{3}(F)} \stackrel{\overline{\delta_{4}}}{\longrightarrow} D_{3}\left(F^{3}\right)_{G L_{3}(F)}\right) \text {. }
$$

LEMA 4.8. Para todo $a, x \in F^{\times}-\{1\}$, em $\mathfrak{p}(F)$ temos $[a]+[1-a]=[x]+[1-x]$. Em particular, o elemento $c_{F}:=[a]+[1-a] \in B(F)$ é independente da escolha de a $\in F^{\times}-\{1\}$.

Demonstração. Seja $a \neq x$, então em $\mathfrak{p}(F)$, temos

$$
\begin{gathered}
{[a]-[x]+\left[\frac{x}{a}\right]-\left[\frac{1-a^{-1}}{1-x^{-1}}\right]+\left[\frac{1-a}{1-x}\right]=0,} \\
{[1-x]-[1-a]+\left[\frac{x}{a}\right]-\left[\frac{1-a^{-1}}{1-x^{-1}}\right]+\left[\frac{1-a}{1-x}\right]=0 .}
\end{gathered}
$$

Substraindo obtemos: $[x]+[1-x]=[a]+[1-a]$.

Defina assim o homomorfismo

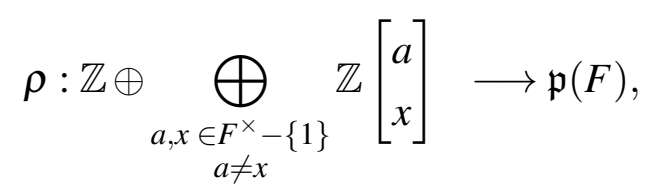

como sendo

$$
\rho(1)=2 c_{F}, \quad \rho\left(\left[\begin{array}{l}
a \\
x
\end{array}\right]\right)=[a] .
$$

Assim temos

$$
\begin{aligned}
\rho \circ \overline{\delta_{4}}\left(\left[\begin{array}{l}
a \\
x
\end{array}\right]\right) & =\rho\left(-1+\left[\begin{array}{c}
\frac{x-a}{x} \\
\frac{x-a}{x-1}
\end{array}\right]+\left[\begin{array}{c}
x^{-1} \\
\frac{x-a}{x(1-a)}
\end{array}\right]+\left[\begin{array}{c}
\frac{x-1}{x-a} \\
a^{-1}
\end{array}\right]+\left[\begin{array}{c}
\frac{x(1-a)}{x-a} \\
\frac{x}{x-a}
\end{array}\right]+\left[\begin{array}{l}
a \\
x
\end{array}\right]\right) \\
& =-2 c_{F}+\left[\frac{x-a}{x}\right]+\left[x^{-1}\right]+\left[\frac{x-1}{x-a}\right]+\left[\frac{x(1-a)}{x-a}\right]+[a] \\
& =-2 c_{F}+c_{F}-\left[\frac{a}{x}\right]+\left[x^{-1}\right]+c_{F}-\left[\frac{1-a}{x-a}\right]+\left[\frac{x(1-a)}{x-a}\right]+[a] \\
& =[a]-\left[\frac{a}{x}\right]+\left[x^{-1}\right]-\left[\frac{1-a}{x-a}\right]+\left[\frac{x(1-a)}{x-a}\right] \\
& =[a]-\left[a x^{-1}\right]+\left[\frac{a x^{-1}}{a}\right]-\left[\frac{1-a^{-1}}{1-\left(a x^{-1}\right)^{-1}}\right]+\left[\frac{1-a}{1-a x^{-1}}\right]=0 \in \mathfrak{p}(F) .
\end{aligned}
$$


e

$$
\begin{aligned}
\rho \circ \overline{\delta_{4}}\left(\left[\begin{array}{ll}
a & b \\
x & y
\end{array}\right]\right) & =\rho\left(\left[\begin{array}{c}
\frac{1-a}{1-b} \\
\frac{(1-y)(1-a)}{(1-x)(1-b)}
\end{array}\right]-\left[\begin{array}{c}
\frac{1-a^{-1}}{1-b^{-1}} \\
\frac{(1-a)(y-b)}{(1-b)(x-a)}
\end{array}\right]+\left[\begin{array}{l}
\frac{b}{a} \\
\frac{y}{x}
\end{array}\right]-\left[\begin{array}{l}
b \\
y
\end{array}\right]+\left[\begin{array}{l}
a \\
x
\end{array}\right]\right) \\
& =\left[\frac{1-a}{1-b}\right]-\left[\frac{1-a^{-1}}{1-b^{-1}}\right]+\left[\frac{b}{a}\right]-[b]+[a]=0 \in \mathfrak{p}(F) .
\end{aligned}
$$

Portanto $\rho$ induz um homomorfismo bem definido $H_{3}\left(D_{\bullet}\left(F^{3}\right)_{G L_{3}(F)}\right) \stackrel{\rho^{\prime}}{\rightarrow} \mathfrak{p}(F)$. Denotemos por $\bar{\rho}$ a composição do homomorfismo $\rho^{\prime}$ com o homorfismo (4.18)

$$
\bar{\rho}=\rho^{\prime} \circ \theta: H_{3}\left(G L_{3}(F)\right) \longrightarrow \mathfrak{p}(F) .
$$

Por outro lado, pelo Exemplo (2.4) temos a sequência exata $C_{\bullet}\left(F^{2}\right) \rightarrow \mathbb{Z}$ de $G L_{2}(F)$-módulos tal que $C_{\bullet}\left(F^{2}\right)_{G L_{2}(F)}$ tem a forma

$$
\cdots \longrightarrow R(F) \stackrel{d_{4,0}^{1}}{\longrightarrow} Q(F) \stackrel{d_{3,0}^{1}=0}{\longrightarrow} \mathbb{Z} \stackrel{d_{2,0}^{1}=i d_{\mathbb{Z}}}{\longrightarrow} \mathbb{Z} \stackrel{d_{1,0}^{1}=0}{\longrightarrow} \mathbb{Z}
$$

e portanto $\mathfrak{p}(F)=H_{3}\left(C_{\bullet}\left(F^{2}\right)_{G L_{2}(F)}\right)$. Pelo Teorema (1.3), existe um morfismo de complexos de cadeias, único a menos de homotopia, $\alpha_{\bullet}:\left(B_{\bullet}\left(G L_{3}(F)\right), d_{\bullet}\right) \rightarrow\left(C_{\bullet}\left(F^{2}\right), \partial_{\bullet}\right)$ tal que $\alpha_{\bullet} \otimes i d_{\mathbb{Z}}:$ $B_{\bullet}\left(G L_{3}(F)\right)_{G L_{2}(F)} \rightarrow C_{\bullet}\left(F^{2}\right)_{G L_{2}(F)}$ é uma equivalência fraca e temos assim o homomorfismo:

$$
\varphi: H_{3}\left(G L_{2}(F)\right)=H_{3}\left(B \bullet\left(G L_{3}(F)\right)_{G L_{2}(F)}\right) \longrightarrow H_{3}\left(C_{\bullet}\left(F^{2}\right)_{G L_{2}(F)}\right)=\mathfrak{p}(F) .
$$

Logo a sequência (4.12) é dada por

$$
H_{3}\left(G M_{2}(F)\right) \rightarrow H_{3}\left(G L_{2}(F)\right) \stackrel{\varphi}{\rightarrow} B(F) \rightarrow 0 .
$$

Proposição 4.1. Seja F um corpo infinito. Então o seguinte diagrama comuta

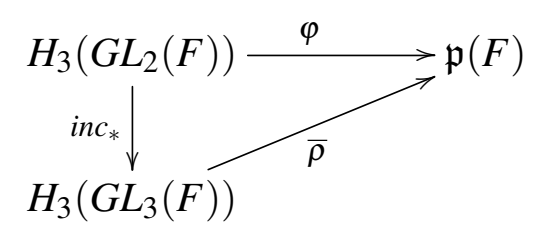

onde inc $: G L_{2}(F) \rightarrow G L_{3}(F)$ é dada por $A \mapsto\left(\begin{array}{cc}A & 0 \\ 0 & 1\end{array}\right)$ e $\varphi$ foi construida em (4.20).

Demonstração. Para $n \geq 0$ defina $C_{n}^{b}\left(F^{3}\right)$ como sendo o subgrupo de $C_{n+1}^{a}\left(F^{3}\right)$ gerado por todos os elementos da forma $\left(\left\langle v_{0}\right\rangle,\left\langle v_{1}\right\rangle, \ldots,\left\langle v_{n-1}\right\rangle,\left\langle e_{3}\right\rangle\right)$. Para $n \geq 1$,

$$
\partial_{n+1}^{a}\left(\left\langle v_{0}\right\rangle, \ldots,\left\langle v_{n-1}\right\rangle,\left\langle e_{3}\right\rangle\right)=\sum_{i=0}^{n}(-1)^{i}\left(\left\langle v_{0}\right\rangle, \ldots, \widehat{\left\langle v_{i}\right\rangle}, \ldots,\left\langle v_{n-1}\right\rangle,\left\langle e_{3}\right\rangle\right) \in C_{n-1}^{b}\left(F^{3}\right),
$$

$\operatorname{logotemos} \partial_{n+1}^{a}\left(C_{n}^{b}\left(F^{3}\right)\right) \subseteq C_{n-1}^{b}\left(F^{3}\right)$. Para $n \geq 1$, seja $\partial_{n}^{b}:=\left.\partial_{n+1}^{a}\right|_{C_{n}^{b}\left(F^{3}\right)}: C_{n}^{b}\left(F^{3}\right) \rightarrow C_{n-1}^{b}\left(F^{3}\right)$, e sejam $C_{-1}^{b}\left(F^{3}\right)=\mathbb{Z}$ e $\partial_{0}^{b}:=\left.\varepsilon_{D}\right|_{C_{0}^{b}\left(F^{3}\right)}$. Além disso, note que $C_{n}^{b}\left(F^{3}\right) \subseteq D_{n}^{b}\left(F^{3}\right)$ para todo $n$. Para $n \geq 0$, vamos a definir uma ação de $G L_{2}(F)$ sobre $C_{n}^{b}\left(F^{3}\right)$ como

$$
\begin{aligned}
& A \cdot\left(\left\langle v_{0}\right\rangle, \ldots,\left\langle v_{n}\right\rangle,\left\langle e_{3}\right\rangle\right):=A^{\prime} \cdot\left(\left\langle v_{0}\right\rangle, \ldots,\left\langle v_{n}\right\rangle,\left\langle e_{3}\right\rangle\right) \\
& A \cdot\left(\left\langle v_{0}\right\rangle, \ldots,\left\langle v_{n}\right\rangle,\left\langle e_{3}\right\rangle\right):=\left(\left\langle A^{\prime} v_{0}\right\rangle, \ldots,\left\langle A^{\prime} v_{n}\right\rangle,\left\langle A^{\prime} e_{3}\right\rangle\right)=\left(\left\langle A^{\prime} v_{0}\right\rangle, \ldots,\left\langle A^{\prime} v_{n}\right\rangle,\left\langle e_{3}\right\rangle\right) \in C_{n}^{b}\left(F^{3}\right) .
\end{aligned}
$$


onde $A \in G L_{2}(F)$ e $A^{\prime}=\left(\begin{array}{cc}A & 0 \\ 0 & 1\end{array}\right) \in G L_{3}(F)$. Obtemos assim um $G L_{2}(F)$-subcomplexo de $D_{\bullet}\left(F^{3}\right) \rightarrow \mathbb{Z}$

$$
C_{\bullet}^{b}\left(F^{3}\right) \rightarrow \mathbb{Z}: \cdots \rightarrow C_{n}^{b}\left(F^{3}\right) \stackrel{\partial_{n}^{b}}{\longrightarrow} C_{n-1}^{b}\left(F^{3}\right) \longrightarrow \cdots \rightarrow C_{1}^{b}\left(F^{3}\right) \stackrel{\partial_{1}^{b}}{\longrightarrow} C_{0}^{b}\left(F^{3}\right) \stackrel{\partial_{0}^{b}}{\longrightarrow} \mathbb{Z} \rightarrow 0 .
$$

De forma similiar aos Lemas (4.6) e (4.7), podemos provar que $C_{\bullet}^{b}\left(F^{3}\right) \rightarrow \mathbb{Z}$ é exata. Como $B .\left(G L_{3}(F)\right) \rightarrow \mathbb{Z}$ é uma resolução livre de $\mathbb{Z}$ sobre $G L_{2}(F)$, então existe um morfismo de complexos, único a menos de homotopia, $\left(B_{\bullet}\left(G L_{3}\right), d_{\bullet}\right) \rightarrow\left(C_{\bullet}^{b}\left(F^{3}\right), \partial_{\bullet}^{b}\right)$. Logo temos a composição dos homomorfismos

$$
H_{3}\left(G L_{2}(F)\right)=H_{3}\left(B_{\bullet}\left(G L_{3}(F)\right)_{G L_{2}(F)}\right) \longrightarrow H_{3}\left(C_{\bullet}^{b}\left(F^{3}\right)_{G L_{2}(F)}\right) \longrightarrow H_{3}\left(D_{\bullet}\left(F^{3}\right)_{G L_{3}(F)}\right)
$$

Considerando o diagrama comutativo

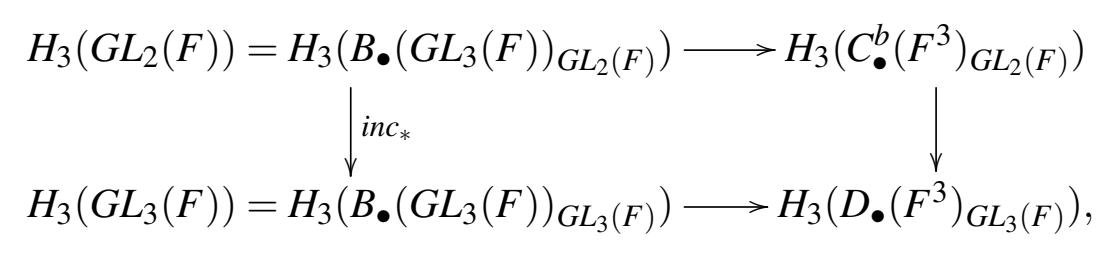

a composição dos homomorfismos

$$
H_{3}\left(G L_{2}(F)\right) \longrightarrow H_{3}\left(G L_{3}(F)\right) \longrightarrow H_{3}\left(D_{\bullet}\left(F^{3}\right)_{G L_{3}(F)}\right) \stackrel{\rho}{\longrightarrow} \mathfrak{p}(F),
$$

pode ser escrita como sendo

$$
H_{3}\left(G L_{2}(F)\right) \longrightarrow H_{3}\left(C_{\bullet}^{b}\left(F^{3}\right)_{G L_{2}(F)}\right) \longrightarrow H_{3}\left(D_{\bullet}\left(F^{3}\right)_{G L_{3}(F)}\right) \stackrel{\rho}{\longrightarrow} \mathfrak{p}(F) .
$$

É fácil verificar que $\varphi$ coincide com a composição (4.24). Por outro lado, a projeção $\pi: F^{3} \rightarrow F^{2}$ dada por $\pi(a, b, c)=(a, b)$ induz um $G L_{2}(F)$-morfismo de complexos $\pi_{\bullet}: C_{\bullet}^{b}\left(F^{3}\right) \rightarrow C_{\bullet}\left(F^{2}\right)$ dado por

$$
\pi_{n}\left(\left\langle v_{0}\right\rangle, \ldots,\left\langle v_{n}\right\rangle,\left\langle e_{3}\right\rangle\right)=\left(\left\langle\pi\left(v_{0}\right)\right\rangle, \ldots,\left\langle\pi\left(v_{n}\right)\right\rangle\right) .
$$

É fácil verificar que o diagrama

$$
\begin{array}{r}
H_{3}\left(G L_{2}(F)\right)=H_{3}\left(B_{\bullet}\left(G L_{3}(F)\right)_{G L_{2}(F)}\right) \longrightarrow \\
H_{3}(C \bullet \\
\left.\downarrow_{\bullet}\left(F^{2}\right)_{G L_{2}(F)}\right)=\mathfrak{p}(F)
\end{array}
$$

é comutativo. Considere agora o diagrama

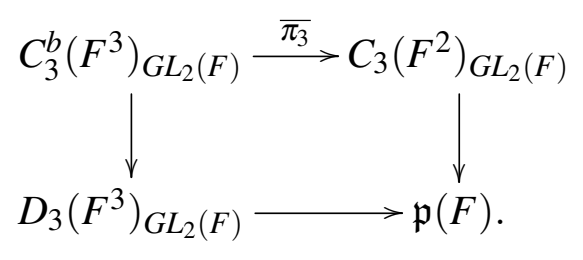


Se $x=\left(\left\langle v_{0}\right\rangle,\left\langle v_{1}\right\rangle,\left\langle v_{2}\right\rangle,\left\langle v_{3}\right\rangle,\left\langle e_{3}\right\rangle\right) \in C_{3}^{b}\left(F^{3}\right)$, então existe $A \in G L_{2}(F)$ tal que

$$
A \cdot\left(\left\langle e_{1}+r e_{3}\right\rangle,\left\langle e_{2}+s e_{3}\right\rangle,\left\langle e_{1}+e_{2}+t e_{3}\right\rangle,\left\langle e_{1}+a e_{2}+u e_{3}\right\rangle,\left\langle e_{3}\right\rangle\right)=x,
$$

onde $a \in F^{\times}-\{1\}, r, s, t, u \in F$. Considere

$$
x^{\prime}=\left(\left\langle e_{1}+r e_{3}\right\rangle,\left\langle e_{2}+s e_{3}\right\rangle,\left\langle e_{1}+e_{2}+t e_{3}\right\rangle,\left\langle e_{1}+a e_{2}+u e_{3}\right\rangle,\left\langle e_{3}\right\rangle\right) \in C_{3}^{b}\left(F^{3}\right)
$$

e note que

$$
\pi_{3}\left(x^{\prime}\right)=\left(\left\langle e_{1}\right\rangle,\left\langle e_{2}\right\rangle,\left\langle e_{1}+e_{2}\right\rangle,\left\langle e_{1}+a e_{2}\right\rangle\right) \in C_{3}\left(F^{2}\right) .
$$

Isto implica que a órbita do elemento $x$ em $C_{3}^{b}\left(F^{2}\right)_{G L_{2}(F)}$ vai para o elemento $[a] \in \mathfrak{p}(F)$. Como existe $A^{\prime} \in G L_{3}(F)$ tal que

$$
A^{\prime} \cdot\left(\left\langle e_{1}\right\rangle,\left\langle e_{2}\right\rangle,\left\langle e_{1}+e_{2}+e_{3}\right\rangle,\left\langle e_{1}+a e_{2}+w e_{3}\right\rangle,\left\langle e_{3}\right\rangle\right)=x^{\prime},
$$

para algum $w \in F^{\times}-\{1\}, a \neq w$, a imagem da órbita de $x$ em $D_{3}\left(F^{3}\right)_{G L_{3}(F)}$ coincide com a órbita do elemento $\left[\begin{array}{l}a \\ w\end{array}\right]$. Aplicando o homomorfismo $\rho$ neste elemento temos que órbita de $x$ em $D_{3}\left(F^{3}\right)_{G L_{2}(F)}$ vai para o elemento $[a] \in \mathfrak{p}(F)$. Portanto obtemos o seguinte diagrama comutativo

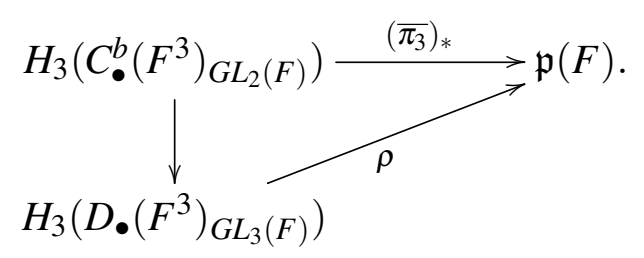

Portanto o seguinte diagrama comuta

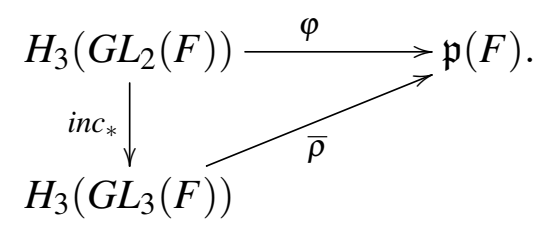

\subsection{A sequência exata de Bloch-Wigner}

TEOREMA 4.2 (Suslin). Seja F um corpo infinito. Então temos a seguinte sequência exata

$$
H_{3}\left(G M_{2}(F)\right) \oplus H_{3}\left(T_{3}\right) \longrightarrow H_{3}\left(G L_{3}(F)\right) \stackrel{\bar{\rho}}{\longrightarrow} B(F) \rightarrow 0 .
$$

Demonstração. O homomorfismo de augmentação $\varepsilon_{D}: D_{0}\left(F^{3}\right) \rightarrow \mathbb{Z}$, da resolução $D_{\bullet}\left(F^{3}\right)$, tem uma seção $T_{3}$-equivariante dada por

$$
\begin{aligned}
\mathbb{Z} & \longrightarrow D_{0}\left(F^{3}\right), \\
n & \longmapsto n\left(\left\langle e_{1}\right\rangle,\left\langle e_{2}\right\rangle\right),
\end{aligned}
$$


onde $T_{3}:=\left\{\operatorname{diag}(a, b, c) \in G L_{3}(F): a, b, c \in F^{\times}\right\} \cong F^{\times} \times F^{\times} \times F^{\times}$. As sequências exatas

$$
\begin{gathered}
0 \rightarrow \operatorname{Im} \partial_{1} \rightarrow D_{0}\left(F^{3}\right) \rightarrow \mathbb{Z} \rightarrow 0, \\
0 \rightarrow \operatorname{Im} \partial_{2} \rightarrow D_{1}\left(F^{3}\right) \rightarrow \operatorname{Im} \partial_{1} \rightarrow 0, \\
0 \rightarrow \operatorname{Im} \partial_{3} \rightarrow D_{2}\left(F^{3}\right) \rightarrow \operatorname{Im} \partial_{2} \rightarrow 0
\end{gathered}
$$

implicam os homomorfismos conectantes

$$
H_{3}\left(T_{3}\right) \rightarrow H_{2}\left(T_{3}, \operatorname{Im} \delta_{1}\right) \rightarrow H_{1}\left(T_{3}, \operatorname{Im} \delta_{2}\right) \rightarrow H_{0}\left(T_{3}, \operatorname{Im} \delta_{3}\right)
$$

Como a primera sequência cinde, $H_{3}\left(T_{3}\right) \rightarrow H_{2}\left(T_{3}\right.$, Im $\left.\partial_{1}\right)$ é trivial. Agora considerando o diagrama comutativo

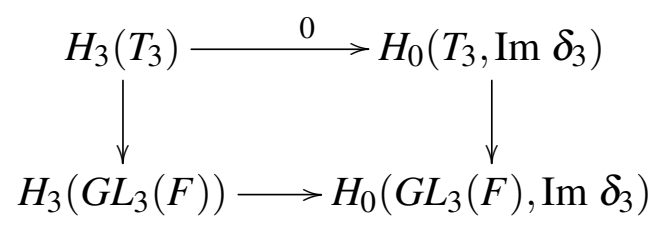

e usando o fato que $H_{0}\left(G L_{3}(F), \operatorname{Im} \delta_{3}\right)=\frac{\operatorname{Ker} \overline{\delta_{3}}}{\operatorname{Im} \overline{\delta_{4}}}=H_{3}\left(D_{\bullet}\left(F^{3}\right)_{G L_{3}(F)}\right)$, então obtemos

$$
\left(\operatorname{inc}_{1}\right)_{*}\left(H_{3}\left(T_{3}\right)\right) \subseteq \operatorname{Ker} \bar{\rho}
$$

onde $i n c_{1}: T_{3} \rightarrow G L_{3}(F)$ é a inclusão de $T_{3}$ em $G L_{3}(F)$. Agora, seja $x \in H_{3}\left(G L_{3}(F)\right)$. Então existem $y \in H_{3}\left(T_{3}\right), z \in H_{3}\left(G L_{2}(F)\right)$ tais que

$$
x=\left(i n c_{1}\right)_{*}(y)+i n c_{*}(z) .
$$

Como a imagem de $H_{3}\left(T_{3}\right)$ está contido no $\operatorname{Ker} \bar{\rho}$, temos

$$
\bar{\rho}(x)=\bar{\rho}\left(i n c_{*}(z)\right)=\bar{\rho} \circ i n c_{*}(z)=\varphi(z) \in B(F) .
$$

Isto junto com a sequência (4.21) prova a exatidão em $B(F)$. Seja agora $x \in H_{3}\left(G L_{3}(F)\right)$ tal que $\bar{\rho}(x)=0$. Então existem $y \in H_{3}\left(T_{3}\right), z \in H_{3}\left(G L_{2}(F)\right)$ tais que $x=\left(i n c_{1}\right)_{*}(y)+i n c_{*}(z)$. Logo $0=\bar{\rho}(x)=\bar{\rho} \circ i n c_{*}(z)=\varphi(z)$. Agora pela sequência (4.21) existe $z^{\prime} \in H_{3}\left(G M_{2}(F)\right)$ tal que $z^{\prime} \mapsto z$. Isto prova a exatidão em $H_{3}\left(G L_{3}(F)\right)$.

O homomorfismo $v: G L(R) \rightarrow S L(R)$ dado por $A \mapsto\left(\begin{array}{cc}\operatorname{det}(A)^{-1} & 0 \\ 0 & A\end{array}\right)$, induz um homomorfismo $\nu_{*}: H_{n}(G L(R)) \rightarrow H_{n}(S L(R))$ tal que a composição

$$
H_{n}(S L(R)) \stackrel{i_{*}}{\longrightarrow} H_{n}(G L(R)) \stackrel{v_{*}}{\longrightarrow} H_{n}(S L(R)),
$$

é o homomorfismo identidade, onde $i: S L(R) \rightarrow G L(R)$ é a inclusão de $S L(R)$ em $G L(R)$. Assim o homomorfismo natural $H_{n}(S L(R)) \stackrel{i_{*}}{\longrightarrow} H_{n}(G L(R))$ é sempre uma injeção que cinde. Com esta última observação temos as ferramentas necessárias para apresentar nosso teorema principal. 
TEOREMA 4.3 (Suslin-Bloch-Wigner). Seja F um corpo infinito. Então existe uma sequência exata

$$
\operatorname{Tor}_{1}^{\mathbb{Z}}(\mu(F), \mu(F))^{\sim} \longrightarrow K_{3}^{\text {ind }}(F) \longrightarrow B(F) \longrightarrow 0
$$

Demonstração. Pela Fórmula de Künneth, $H_{3}\left(T_{3}\right) \cong M^{\prime} \oplus\left(F^{\times} \otimes_{\mathbb{Z}} F^{\times} \otimes_{\mathbb{Z}} F^{\times}\right)$, onde $M^{\prime}$ é formado por somandos de $H_{3}\left(T_{2} \times 1\right), H_{3}\left(F^{\times} \times 1 \times F^{\times}\right)$e $H_{3}\left(1 \times T_{2}\right)$. Considere os automorfismos de $H_{3}\left(G L_{3}(F)\right)$ induzidos por conjugação pelas matrices $A_{1}=\left(\begin{array}{lll}0 & 1 & 0 \\ 1 & 0 & 0 \\ 0 & 0 & 1\end{array}\right)$ e $A_{2}=\left(\begin{array}{lll}0 & 0 & 1 \\ 1 & 0 & 0 \\ 0 & 1 & 0\end{array}\right)$ como foi feito em (4.5). Note que em particular para $a_{i}, b_{i} \in F^{\times}$, em $B_{3}\left(G L_{3}(F)\right)$ temos

$$
\begin{gathered}
\left.\left.\tau_{A_{1}}\left(\left|\left(\begin{array}{ccc}
a_{1} & 0 & 0 \\
0 & 1 & 0 \\
0 & 0 & b_{1}
\end{array}\right)\right|\left(\begin{array}{ccc}
a_{2} & 0 & 0 \\
0 & 1 & 0 \\
0 & 0 & b_{2}
\end{array}\right) \mid\left(\begin{array}{ccc}
a_{3} & 0 & 0 \\
0 & 1 & 0 \\
0 & 0 & b_{3}
\end{array}\right)\right]\right)=\left[\left(\begin{array}{ccc}
1 & 0 & 0 \\
0 & a_{1} & 0 \\
0 & 0 & b_{1}
\end{array}\right)\left|\left(\begin{array}{ccc}
1 & 0 & 0 \\
0 & a_{2} & 0 \\
0 & 0 & b_{2}
\end{array}\right)\right| \mid \begin{array}{ccc}
1 & 0 & 0 \\
0 & a_{3} & 0 \\
0 & 0 & b_{3}
\end{array}\right)\right] \\
\tau_{A_{2}}\left(\left[\left(\begin{array}{ccc}
a_{1} & 0 & 0 \\
0 & b_{1} & 0 \\
0 & 0 & 1
\end{array}\right)\left|\left(\begin{array}{ccc}
a_{2} & 0 & 0 \\
0 & b_{2} & 0 \\
0 & 0 & 1
\end{array}\right)\right|\left(\begin{array}{ccc}
a_{3} & 0 & 0 \\
0 & b_{3} & 0 \\
0 & 0 & 1
\end{array}\right)\right]\right)=\left[\left(\begin{array}{ccc}
1 & 0 & 0 \\
0 & a_{1} & 0 \\
0 & 0 & b_{1}
\end{array}\right)\left|\left(\begin{array}{ccc}
1 & 0 & 0 \\
0 & a_{2} & 0 \\
0 & 0 & b_{2}
\end{array}\right)\right|\left(\begin{array}{ccc}
1 & 0 & 0 \\
0 & a_{3} & 0 \\
0 & 0 & b_{3}
\end{array}\right)\right] .
\end{gathered}
$$

Então

$$
\begin{aligned}
& \operatorname{Im}\left(H_{3}\left(F^{\times} \times 1 \times F^{\times}\right) \rightarrow H_{3}\left(G L_{3}(F)\right)\right)=\operatorname{Im}\left(H_{3}\left(T_{2}\right) \rightarrow H_{3}\left(G L_{3}(F)\right)\right), \\
& \operatorname{Im}\left(H_{3}\left(1 \times F^{\times} \times F^{\times}\right) \rightarrow H_{3}\left(G L_{3}(F)\right)\right)=\operatorname{Im}\left(H_{3}\left(T_{2}\right) \rightarrow H_{3}\left(G L_{3}(F)\right)\right) .
\end{aligned}
$$

Isto implica que

$$
\operatorname{Im}\left(M^{\prime} \rightarrow H_{3}\left(G L_{3}(F)\right)\right)=\operatorname{Im}\left(H_{3}\left(T_{2}\right) \rightarrow H_{3}\left(G L_{3}(F)\right)\right) .
$$

Assim,

$$
\operatorname{Im}\left(H_{3}\left(T_{3}\right) \rightarrow H_{3}\left(G L_{3}(F)\right)\right)=\operatorname{Im}\left(H_{3}\left(T_{2}\right) \oplus\left(F^{\times} \otimes_{\mathbb{Z}} F^{\times} \otimes_{\mathbb{Z}} F^{\times}\right) \rightarrow H_{3}\left(G L_{3}(F)\right)\right),
$$

obtendo pelo Teorema (4.2) a sequência exata

$$
H_{3}\left(G M_{2}(F)\right) \oplus\left(F^{\times} \otimes_{\mathbb{Z}} F^{\times} \otimes_{\mathbb{Z}} F^{\times}\right) \longrightarrow H_{3}\left(G L_{3}(F)\right) \stackrel{\bar{\rho}}{\longrightarrow} B(F) \rightarrow 0 .
$$

Agora da decomposição (4.10) e do Teorema (4.2), implica-se a seguinte sequência exata

$$
F_{2}^{\prime} H_{3}\left(G M_{2}(F)\right) \longrightarrow H_{3}\left(G L_{3}(F)\right) / N \longrightarrow B(F) \longrightarrow 0
$$

onde $N:=\operatorname{Im}\left(\left(F^{\times} \otimes_{\mathbb{Z}} F^{\times} \otimes_{\mathbb{Z}} F^{\times}\right) \rightarrow H_{3}\left(G L_{3}(F)\right)\right)$. Pelo Lema (1.5), temos a decomposição canônica $H_{3}\left(T_{2}\right) \cong M \oplus \operatorname{Tor}_{1}^{\mathbb{Z}}(\mu(F), \mu(F))$. Pelo diagrama comutativo

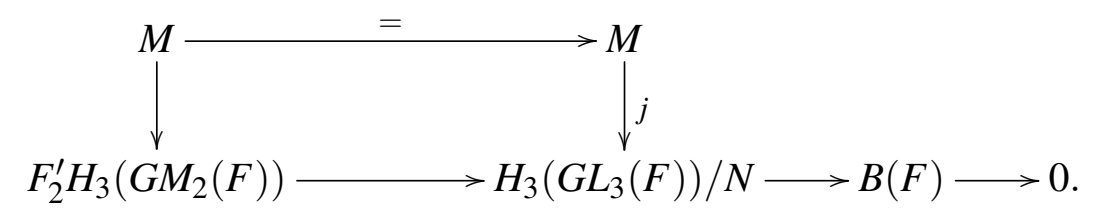

obtemos a sequência exata

$$
T_{F} \longrightarrow \frac{H_{3}\left(G L_{3}(F)\right)}{j(M)+N} \longrightarrow B(F) \longrightarrow 0
$$


onde nos já estudamos $T_{F}$ no Capítulo 1 no Exemplo (1.22). Como pelo Lema (4.4) $T_{F} \cong$ $\operatorname{Tor}_{1}^{\mathbb{Z}}(\mu(F), \mu(F))^{\sim}$ e pelo Teorema de Estabilidade (1.16) $H_{3}\left(G L_{3}(F)\right) \cong H_{3}(G L(F))$, obtemos a sequência exata

$$
\operatorname{Tor}_{1}^{\mathbb{Z}}(\mu(F), \mu(F))^{\sim} \longrightarrow \frac{H_{3}(G L(F))}{j(M)+N} \longrightarrow B(F) \longrightarrow 0 .
$$

Por outro lado, dado que $H_{2}(S L(F)) \cong K_{2}^{M}(F)$ (2.9) e pela sequência (1.15) obtemos a seguinte sequência exata que cinde

$$
0 \longrightarrow F^{\times} \otimes_{\mathbb{Z}} K_{2}^{M}(F) \stackrel{\eta}{\longrightarrow} \frac{H_{3}(G L(F))}{H_{3}\left(F^{\times}\right)} \stackrel{v_{*}}{\longrightarrow} H_{3}(S L(F)) \longrightarrow 0,
$$

onde $v_{*}$ é induzido pelo morfismo $v: G L(F) \rightarrow S L(F), A \mapsto \operatorname{diag}\left(\operatorname{det}(A)^{-1}, A\right)$ enquanto o homomorfismo restante $\eta: F^{\times} \otimes_{\mathbb{Z}} K_{2}^{M}(F) \longrightarrow \frac{H_{3}(G L(F))}{H_{3}\left(F^{\times}\right)}$é definido como sendo a composta

$$
\begin{gathered}
F^{\times} \otimes_{\mathbb{Z}} K_{2}^{M}(F) \longrightarrow H_{1}\left(F^{\times}\right) \otimes_{\mathbb{Z}} H_{2}\left(G L_{2}(F)\right) \longrightarrow H_{3}\left(F^{\times} \times G L_{2}(F)\right) \rightarrow H_{3}\left(G L_{3}(F)\right) \cong H_{3}(G L(F)) \\
x \otimes\{y, z\} \longmapsto x \otimes \mathbf{c}\left(\operatorname{diag}(y, 1), \operatorname{diag}\left(z, z^{-1}\right)\right) \longmapsto \mathbf{c}\left(\operatorname{diag}(x, 1,1), \operatorname{diag}(1, y, 1), \operatorname{diag}\left(1, z, z^{-1}\right)\right)
\end{gathered}
$$

Notemos que para $x, y, z \in F^{\times}$,

$$
\begin{aligned}
v_{*}(\mathbf{c}(\operatorname{diag}(x, 1), \operatorname{diag}(1, y), \operatorname{diag}(1, z)))= & \mathbf{c}\left(\operatorname{diag}\left(x^{-1}, x, 1\right), \operatorname{diag}\left(y^{-1}, 1, y\right), \operatorname{diag}\left(z^{-1}, 1, z\right)\right) \\
= & -\mathbf{c}\left(\operatorname{diag}\left(x, x^{-1}, 1\right), \operatorname{diag}\left(y, 1, y^{-1}\right), \operatorname{diag}\left(z, 1, z^{-1}\right)\right) \\
= & \mathbf{c}\left(\operatorname{diag}\left(y, 1, y^{-1}\right), \operatorname{diag}\left(x, x^{-1}, 1\right), \operatorname{diag}\left(z, 1, z^{-1}\right)\right) \\
= & {[y, x, z], } \\
v_{*}(\mathbf{c}(\operatorname{diag}(x, 1,1), \operatorname{diag}(y, 1,1), \operatorname{diag}(1,1, z)))= & \mathbf{c}\left(\operatorname{diag}\left(x^{-1}, x, 1,1\right), \operatorname{diag}\left(y^{-1}, y, 1,1\right), \operatorname{diag}\left(z^{-1}, 1,1, z\right)\right) \\
= & -\mathbf{c}\left(\operatorname{diag}\left(x, x^{-1}, 1,1\right), \operatorname{diag}\left(y, y^{-1}, 1,1\right), \operatorname{diag}\left(z, 1,1, z^{-1}\right)\right) \\
= & -\mathbf{c}\left(\operatorname{diag}\left(x, 1, x^{-1}, 1\right), \operatorname{diag}\left(y, 1, y^{-1}, 1\right), \operatorname{diag}\left(z, 1,1, z^{-1}\right)\right) \\
= & -\mathbf{c}\left(\operatorname{diag}\left(x, 1, x^{-1}, 1\right), \operatorname{diag}\left(y, 1, y^{-1}, 1\right), \operatorname{diag}\left(z, z^{-1}, 1,1\right)\right) \\
& -\mathbf{c}\left(\operatorname{diag}\left(x, 1, x^{-1}, 1\right), \operatorname{diag}\left(y, 1, y^{-1}, 1\right), \operatorname{diag}\left(1, z, 1, z^{-1}\right)\right) \\
= & \mathbf{c}\left(\operatorname{diag}\left(x, 1, x^{-1}, 1\right), \operatorname{diag}\left(z, z^{-1}, 1,1\right), \operatorname{diag}\left(y, 1, y^{-1}, 1\right)\right) \\
= & {[x, z, y], } \\
= & \mathbf{c}\left(\operatorname{diag}\left(x^{-1}, x, 1,1\right), \operatorname{diag}\left(y^{-1}, 1, y, 1\right), \operatorname{diag}\left(z^{-1}, 1,1, z\right)\right) \\
= & -\mathbf{c}\left(\operatorname{diag}\left(x, x^{-1}, 1,1\right), \operatorname{diag}\left(y, 1, y^{-1}, 1\right), \operatorname{diag}\left(z, 1,1, z^{-1}\right)\right) \\
= & \mathbf{c}\left(\operatorname{diag}\left(x, x^{-1}, 1,1\right), \operatorname{diag}\left(z, 1, z^{-1}, 1\right), \operatorname{diag}\left(y, 1, y^{-1}, 1\right)\right) \\
& +\mathbf{c}\left(\operatorname{diag}\left(x, x^{-1}, 1,1\right), \operatorname{diag}\left(1,1, z, z^{-1}\right), \operatorname{diag}\left(y, 1, y^{-1}, 1\right)\right) \\
= & \mathbf{c}\left(\operatorname{diag}\left(x, x^{-1}, 1,1\right), \operatorname{diag}\left(z, 1, z^{-1}, 1\right), \operatorname{diag}\left(y, 1, y^{-1}, 1\right)\right) \\
= & {[x, z, y], }
\end{aligned}
$$

isto é, $v_{*}(j(M)+N) \subseteq T$, onde $T=\left\langle[a, b, c]: a, b, c \in F^{\times}\right\rangle$. Assim obtemos um homomorfismo sobrejetor $\overline{V_{*}}: \frac{H_{3}(G L(F))}{j(M)+N} \rightarrow \frac{H_{3}(S L(F))}{T} \cong K_{3}^{\text {ind }}(F)$, onde o último isomorfismo é dado pelo Corolário (3.2). Agora, dado que

$$
\begin{aligned}
\mathbf{c}\left(\operatorname{diag}(x, 1,1), \operatorname{diag}(1, y, 1), \operatorname{diag}\left(1, z, z^{-1}\right)\right) & =\mathbf{c}(\operatorname{diag}(x, 1,1), \operatorname{diag}(1, y, 1), \operatorname{diag}(1, z, 1)) \\
& -\mathbf{c}(\operatorname{diag}(x, 1,1), \operatorname{diag}(1, y, 1), \operatorname{diag}(1,1, z)),
\end{aligned}
$$

temos

$$
\operatorname{Ker} v_{*}=\operatorname{Im} \eta=\eta\left(F^{\times} \otimes_{\mathbb{Z}} K_{2}^{M}(F)\right) \subseteq j(M)+N,
$$


Portanto usando o diagrama comutativo

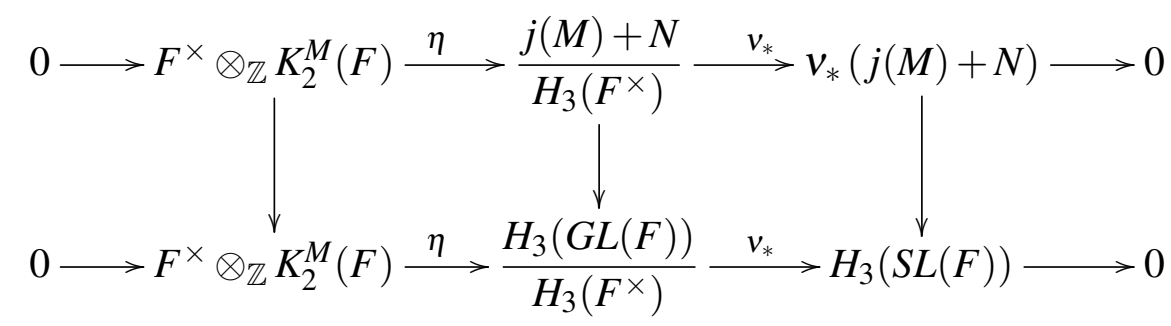

temos o isomorfismo, $\frac{H_{3}(G L(F))}{j(M)+N} \cong K_{3}^{\text {ind }}(F)$ e obtemos a sequência exata (4.25).

Como resultado final, juntando as sequências exatas (4.1) e (4.25), apresentamos a sequência exata de Bloch-Wigner na forma geral.

TEOREMA 4.4 (Bloch-Wigner). Seja F um corpo infinito. Então temos a sequência exata

$$
\operatorname{Tor}_{1}^{\mathbb{Z}}(\mu(F), \mu(F))^{\sim} \longrightarrow K_{3}^{\text {ind }}(F) \longrightarrow \mathfrak{p}(F) \stackrel{\lambda}{\longrightarrow}\left(F^{\times} \otimes_{\mathbb{Z}} F^{\times}\right)_{\sigma} \longrightarrow K_{2}(F) \longrightarrow 0,
$$

onde o homomorfismo $\mathfrak{p}(F) \rightarrow\left(F^{\times} \otimes_{\mathbb{Z}} F^{\times}\right)_{\sigma}$ é dado por $[a] \mapsto a \otimes(1-a)$, e $\operatorname{Tor}_{1}^{\mathbb{Z}}(\mu(F), \mu(F))^{\sim}$ é a única extensão não trivial de $\mathbb{Z} / 2 \mathbb{Z}$ por $\operatorname{Tor}_{1}^{\mathbb{Z}}(\mu(F), \mu(F))$ se char $(F) \neq 2$ e $\mu_{2^{\infty}}(F)$ é finito, e $\operatorname{Tor}_{1}^{\mathbb{Z}}(\mu(F), \mu(F))^{\sim}:=\operatorname{Tor}_{1}^{\mathbb{Z}}(\mu(F), \mu(F))$ caso contrário.

ObSeRVAÇão 4.1. Pode-se provar que o homomorfismo

$$
\operatorname{Tor}_{1}^{\mathbb{Z}}(\mu(F), \mu(F))^{\sim} \longrightarrow K_{3}^{\text {ind }}(F)
$$

é injetor. Mas a demonstração deste fato é difícil e precisamos mostrar alguns resultados adicionais da $K$-teoria algébrica. Pode-se notar também que se $F$ tem um número finito de elementos, existem já alguns resultados parciais para a obtenção de uma sequência exata de Bloch-Wigner para o corpo $F$ (veja-se [6]).

EXEMPLO 4.1. A sequência original para (4.28), demonstrada por Bloch e Wigner separadamente, garante a existência de uma sequência exata da forma

$$
0 \rightarrow \mathbb{Q} / \mathbb{Z} \rightarrow H_{3}\left(S L_{2}(\mathbb{C})\right) \rightarrow \mathfrak{p}(\mathbb{C}) \rightarrow \bigwedge_{\mathbb{Z}}^{2} \mathbb{C}^{\times} \rightarrow K_{2}(\mathbb{C}) \rightarrow 0
$$

onde $\mathfrak{p}(\mathbb{C})$ é o grupo pré-Bloch de $\mathbb{C}, \log 0 H_{3}\left(S L_{2}(\mathbb{C})\right) \cong K_{3}^{\text {ind }}(\mathbb{C})$, isto é obtemos uma descrição da parte indecomponível do terceiro $K$-grupo de $\mathbb{C}$. Pode-se generalizar esta sequência para corpos $k$ algebricamente fechados e de característica zero, obtendo a sequência exata da forma

$$
0 \rightarrow \mathbb{Q} / \mathbb{Z} \rightarrow K_{3}^{\text {ind }}(k) \rightarrow \mathfrak{p}(k) \rightarrow k^{\times} \wedge k^{\times} \rightarrow K_{2}(k) \rightarrow 0 .
$$




\section{REFERÊNCIAS}

1 Brown, K. S. Cohomology of Groups. New York: Springer-Verlag Berlin Heidelberg, 1982. (Graduate Texts in Mathematics, 87). Citado 4 vezes nas páginas 4, 5, 9 e 17.

2 Hatcher, A. Algebraic Topology. United States of America: Cambridge University Press, 2002. Citado 8 vezes nas páginas $69,70,71,72,73,74,75$ e 81.

3 Loday,

École Normale Supérieure, v. 9, p. 309-377, 1976. Citado 3 vezes nas páginas 77, 81 e 82.

4 Mac Lane, S. Homology. Gottingen: Springer-Verlag Berlin Heidelberg, 1963. (Classics in Mathematics, 114). Citado 4 vezes nas páginas 6, 23, 25 e 26.

5 Milnor J. Introduction to Algebraic K-Theory. Princeton, N. J.: Princeton University Press, 1971. (Annals of Mathematics Studies, 72). Citado na página 42.

6 Mirzaii, B. Mokari, Fatemeh. Y. A Bloch-Wigner theorem over rings with many units II. Journal of Pure and Applied Algebra, v. 219, p. 5078-5096, 2015. Citado na página 110.

7 Nesterenko, Y. P.; Suslin, A. A. Homology of the general linear group over a local ring and Milnor's K-theory. Mathematics of the USSR-Izvestiya, v. 34, p. 121-145, 1990. Citado na página 39.

8 Rosenberg, J. Algebraic K-Theory and its applications. New York: Springer-Verlag, 1994. (Graduate Texts in Mathematics, 147). Citado 5 vezes nas páginas 42, 72, 73, 74 e 77.

9 Rotman, J. J. An introduction to homological algebra. Urbana, Illinois: Academic Press, INC, 1979. Citado na página 6.

10 Srinivas, V. Algebraic K-Theory. Birkhauser, Boston: Progress in Mathematics, 90, 1996. Citado na página 75.

11 Suslin, A. A. Homology of $G L_{n}$, characteristic classes and Milnor's K-theory. Proc. Steklov Inst. Math, p. 207-225, 1985. Citado 2 vezes nas páginas 39 e 84.

12 . $K_{3}$ of a field and the Bloch group. Proc. Steklov Inst. Math, p. 217-239, 1991. Citado 2 vezes nas páginas ii e 83.

13 Van der Kallen, W. The $K_{2}$ of rings with many units. Annales scientifiques de l' École Normale Supérieure, v. 10, p. 473-515, 1977. Citado na página i.

14 Vermani, L. R. An elementary approach to homological algebra. Boca Raton, Florida: Chapman \& Hall/CRC, 2003. Citado 3 vezes nas páginas 3, 4 e 6.

15 Weibel, C. A. An Introduction to Homological Algebra. Cambridge, United Kingdom: Cambridge University Press, 1994. (Cambridge Studies in Advanced Mathematics, 38). Citado 5 vezes nas páginas $4,6,7,16$ e 31 . 